\title{
Open Strings in Magnetic Background Fields
}

\author{
D I S S E R T A T I O N \\ zur Erlangung des akademischen Grades \\ doctor rerum naturalium \\ (dr. rer. nat.) \\ im Fach Physik \\ eingereicht an der \\ Mathematisch-Naturwissenschaftlichen Fakultt I \\ Humboldt-Universitt zu Berlin \\ von \\ Herr Dipl.-Phys. Boris Körs \\ geboren am 2.6.1971 in Hamburg
}

Prsident der Humboldt-Universitt zu Berlin:

Prof. Dr. J. Mlynek

Dekan der Mathematisch-Naturwissenschaftlichen Fakultt I:

Prof. Dr. B. Ronacher

Gutachter:

1. Prof. Dr. Dieter Lüst

2. Prof. Dr. Stefan Theisen

3. Prof. Dr. Michael Müller-Preußker

eingereicht am:

5. Mai 2001

Tag der mndlichen Prfung: $\quad$ 24. Juli 2001 


\begin{abstract}
We discuss various aspects of internal magnetic background fields in open string theories. Phenomenologically and conceptually interesting properties of such string theory backgrounds, supersymmetry and gauge symmetry breaking, chiral fermion spectra and noncommutativity of the internal compactification manifolds, are treated in a rather generic framework. We then specialize to type I compactifications on tori and toroidal orbifolds with magnetic fields on the internal space. This allows to develop a strategy for constructing type I vacua with attractive low energy field theories which may either be supersymmetric or not and contain chiral spectra and gauge groups close to the Standard Model or some grand unified generalization thereof. The most sophisticated version uses magnetic fields and NSNS $B$-fields on orbifold spaces giving rise to a plethora of promising examples for semi-realistic string compactifications. We finally also present a related class of asymmetric orbifolds of type I which are of little phenomenological interest but still display certain interesting features. The asymmetric rotations which are gauged in these models identify D-branes with different values for the magnetic field on their world volume, such that the distinction of commutative and noncommutative internal geometries is lost.
\end{abstract}

\title{
Keywords:
}

String vacua, Compactification, Unification, Non-commutative geometry 


\section{Zusammenfassung}

Es werden verschiedene Aspekte interner magnetischer Hintergrundfelder in Theorien offener Strings diskutiert. Phänomenologisch und konzeptionell interessante Eigenschaften solcher Vakua, die Brechung von Supersymmetrie, Eichsymmetrie und chiraler Symmetrie, werden auf ganz generische Weise behandelt. Dann wird eine Spezialisierung auf Typ I Modelle, kompaktifiziert auf Tori und Bahnfaltigkeiten, durchgeführt. Daraus wird eine Methode gewonnen zur Konstruktion von Typ I Vakua mit attraktiven effektiven Feldtheorien als Niederenergienäherungen, sowohl supersymmetrische wie nicht supersymmetrische Modelle mit chiralen Fermionspektren und Eichgruppen ähnlich dem Standardmodell oder einer vereinheitlichenden Verallgemeinerung desselben. Die am weitesten entwickelten Beispiele kombinieren magnetische Felder mit NSNS B-Feldern auf Bahnfaltigkeiten. Zuletzt wird noch eine verwandte Klasse von Modellen besprochen, die zwar eher weniger vielversprechende phänomenologische Perspektiven bietet, aber einige konzeptionelle Spezialitäten aufweist. In diesen Kompaktifizierungen werden asymmetrische Rotationen geeicht, so daß D-branen mit unterschiedlichen Werten für die magnetischen Felder auf ihrem Weltvolumen identifiziert werden, womit die Unterscheidung von kommutativen und nicht kommutativen Geometrien verlorengeht.

\section{Schlagwörter:}

String Vakua, Kompaktifizierung, Vereinheitlichung, Nicht kommutative Geometrie 


\section{Contents}

1 Introduction $\quad 13$

1.1 String theory . . . . . . . . . . . . . . . . . 13

1.2 Compactification . . . . . . . . . . . . . . . . 14

1.3 Dualities . . . . . . . . . . . . . . . . . 16

1.4 Open strings and branes . . . . . . . . . . . . . . . . . . . . . . . . . . . . .

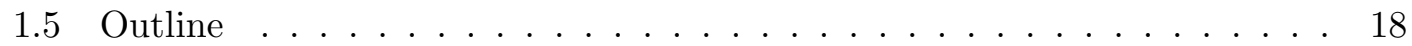

2 Open strings on D-branes with background fluxes 21

2.1 Boundary conformal field theory . . . . . . . . . . . . . . 22

2.1.1 Boundary conditions . . . . . . . . . . . . . . 22

2.1.2 Boundary states for D-branes with flux . . . . . . . . . . . 24

2.2 Chirality and supersymmetry . . . . . . . . . . . . . . . 27

2.2.1 Supersymmetry breaking . . . . . . . . . . . 28

2.2 .2 Chiral fermions . . . . . . . . . . . . . . . . . 29

2.2 .3 Supersymmetric bound states . . . . . . . . . . . . . . . . . . 32

2.3 Noncommutativity and asymmetric rotations . . . . . . . . . . . 33

2.3.1 The commutator of open string coordinates . . . . . . . . . 33

2.3.2 Asymmetric rotations of D-branes . . . . . . . . . . . 36

2.3.3 Two-point function and OPE . . . . . . . . . . . . . 37

2.4 Toroidal compactification . . . . . . . . . . . . . . . 38

2.4.1 Magnetic flux on a torus . . . . . . . . . . . . 39

2.4 .2 Zero-mode spectra . . . . . . . . . . . . . . 40

2.4 .3 T-duality ........................... 42

2.4 Topological data from CFT . . . . . . . . . . . . . . . . . . . 46

2.5 Orbifold compactification . . . . . . . . . . . . 48

3 Type I superstrings with background fluxes 53

3.1 Tadpole cancellation and anomalies . . . . . . . . . . . . . 54

3.1.1 Nonorientable string diagrams . . . . . . . . . . . . . . 54

3.1.2 Space-time anomalies . . . . . . . . . . . . . . . 60

3.2 Large volume compactification . . . . . . . . . . . . . . . . 62

3.3 Toroidal compactification with magnetic flux . . . . . . . . . . . 64

3.3 .1 Construction . . . . . . . . . . . . . . . 65

3.3 .2 Internal geometry . . . . . . . . . . . . . . . . 72

3.3.3 Spectra in six dimensions . . . . . . . . . . . . . . 75

3.3.4 Fourdimensional models . . . . . . . . . . . . . . 77 
3.4 Toroidal compactification with magnetic flux and NSNS $B$-field . . . . . 83

3.4.1 Construction . . . . . . . . . . . . . . . . 84

3.4.2 A left-right symmetrically unified model . . . . . . . . . . . . 86

3.5 Orbifold compactification on K3 with fluxes . . . . . . . . . . . . . 88

3.5.1 Construction . . . . . . . . . . . . . . . 89

3.5.2 Massless spectra . . . . . . . . . . . . . . . . 93

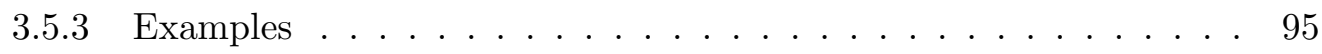

3.6 Further phenomenological perspectives . . . . . . . . . . . . . . 98

4 Type I superstrings with asymmetric symmetries 101

4.1 A class of supersymmetric asymmetric orientifolds . . . . . . . . . . . 102

4.1 .1 Closed strings . . . . . . . . . . . . . . . . . 103

4.1 .2 Open strings . . . . . . . . . . . . . . . . . . 107

4.2 Sixdimensional models . . . . . . . . . . . . . . . . . 110

4.3 Fourdimensional models . . . . . . . . . . . . . . . . . . . . 112

4.3.1 The example $\mathbb{T}^{6} / \mathbb{Z}_{4} \ldots \ldots \ldots \ldots \ldots 113$

4.3 .2 Results for other examples . . . . . . . . . . . . . 115

$\begin{array}{llr}5 & \text { Summary } & 117\end{array}$

$\begin{array}{lr}\text { A Definitions and notation } & 123\end{array}$

$\begin{array}{ll}\text { B Annulus diagrams and modular transformation } & 127\end{array}$

C One-loop partition function in asymmetric orientifolds $\quad 131$

C.1 Klein bottle . . . . . . . . . . . . . . . . . 131

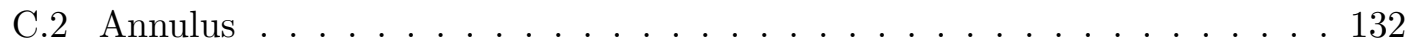

C.3 Möbius strip . . . . . . . . . . . . . . . . . . 133

$\begin{array}{ll}\text { D Useful formulas } & 135\end{array}$ 


\section{List of Figures}

1.1 The string vertex . . . . . . . . . . . . . . . . . . 14

1.2 Perturbative expansion of string scattering . . . . . . . . . . . . . . 14

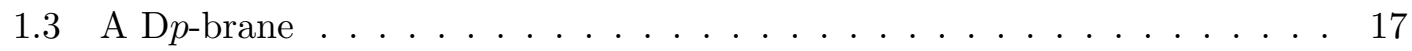

2.1 Open-closed world sheet consistency . . . . . . . . . . . 25

2.2 The generic torus $\mathbb{T}^{2} \ldots \ldots \ldots \ldots \ldots \ldots \ldots$

2.3 T-duality on $X^{2}$ applied to $U$ and $T \ldots \ldots \ldots \ldots \ldots$

2.4 An $(n, m)=(3,1)$ D-brane . . . . . . . . . . . . . 44

2.5 Intersecting $(1,2)$ and $(3,1)$ D-branes $\ldots \ldots \ldots \ldots \ldots$

3.1 Fundamental domains for the annulus, Möbius strip and the Klein bottle 55

3.2 Tree channel one-loop amplitude in type I . . . . . . . . . . . . . . . 59

3.3 Sectors of open strings . . . . . . . . . . . . . . . . . 68

3.4 Configuration of D6-branes . . . . . . . . . . . . 80

3.5 ConfigurationD6-branes . . . . . . . . . . . . . . 82

3.6 T-duals of D5- and D9-branes . . . . . . . . . . . 86

3.7 T-dual torus with $\mathbb{Z}_{2}$ fixed points $\ldots \ldots \ldots \ldots \ldots$

3.8 Configurations of D7-branes . . . . . . . . . . . . . . . 96

$4.1 \quad \mathbf{A}$ and $\mathbf{B}$ type lattices with $\mathbb{Z}_{3}$ symmetry . . . . . . . . . . . . 105

4.2 D-branes wrapping $\mathbf{A}$ and $\mathbf{B}$ type lattices . . . . . . . . . . . . . 109

$4.3 \mathbf{A}$ and $\mathbf{B}$ type lattices with $\mathbb{Z}_{4}$ symmetry $\ldots \ldots \ldots \ldots$ 


\section{List of Tables}

2.1 K3 orbifolds . . . . . . . . . . . . . . . . . . . . . . . . 49

2.2 Calabi-Yau orbifolds . . . . . . . . . . . . . . . . . . . 49

3.1 Chiral massless fermions in $d=6 \ldots \ldots \ldots \ldots$. . . . . . . . . 77

3.2 Chiral massless fermions in $d=4 \ldots \ldots \ldots \ldots$. . . . . . . . . . 78

3.3 Four generation spectrum . . . . . . . . . . . . . . . . 81

3.4 Four generation Standard Model spectrum . . . . . . . . . . . . . . . 81

3.5 GUT spectrum . . . . . . . . . . . . . . . . . 83

3.6 Massless chiral fermions . . . . . . . . . . . . . . . . . 87

3.7 Left-right symmetrically unified spectrum . . . . . . . . . . . . 88

3.8 Closed string spectra . . . . . . . . . . . . . . . . . . . . 94

3.9 Spectra of $\mathbb{Z}_{2}$ orbifold . . . . . . . . . . . . . . . . . 95

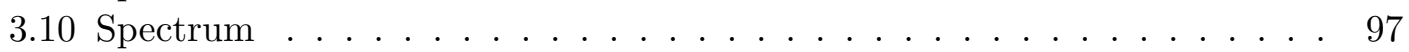

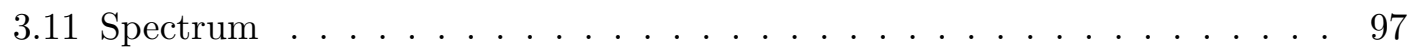

3.12 Spectrum . . . . . . . . . . . . . . . . . . 98

4.1 Closed string spectra in $d=6 \ldots \ldots \ldots \ldots$. . . . . . . . . . . . . . . . .

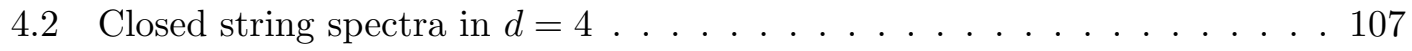

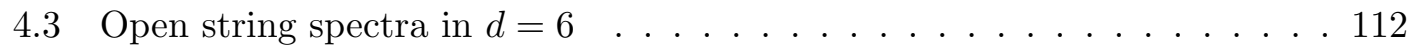

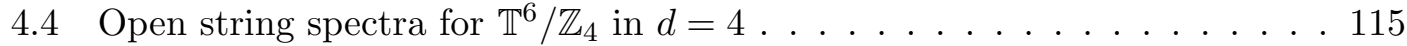

4.5 Open string spectra for $\mathbb{T}^{6} / \mathbb{Z}_{N}, N=3,6,6^{\prime}$ in $d=4 \ldots \ldots \ldots$ 


\section{Chapter 1}

\section{Introduction}

One of the ultimate goals of theoretical high energy physics is a unified understanding of all the four known forces. It should provide an explanation of the particular matter content observed in nature and a dynamical derivation of all parameter values as measured at laboratory energies. A central obstacle in the framework of local quantum field theory is the lack of a completely satisfactory description of the dynamics at very small distances. Quantum effects tend to destabilize the classical vacuum of the Standard Model of electroweak interactions, which can only be resolved by an unacceptable amount of fine tuning. Furthermore, quantum gravity appears to be nonrenormalizable anyway. One way to deal with some of the problems of gauge interactions consists in adding new degrees of freedom slightly above the electroweak scale which by supersymmetry smooth out the quantum effects. The natural generalization of global supersymmetry to local supergravity still has no consistent interpretation at small distances. So one usually employs it as an effective theory for a unified description of gravitational and gauge interactions valid far below the Planck scale. The question which are then the fundamental degrees of freedom emerging at energies near the Planck scale may have different answers. In this thesis they are assumed to be strings, or even branes, their higher dimensional generalizations.

\section{$1.1 \quad$ String theory}

String theory $[1,2,3]$ in its perturbative formulation replaces point-like elementary particles by objects which are extended in one spatial dimension, strings. By propagating through space-time they sweep out their world sheet $\Sigma$. They may split and recombine and their dynamics is governed by a twodimensional conformal field theory, which has the target space coordinates $X^{\mu}$ of $\Sigma$ and, in case of the superstring, their fermionic partners $\Psi^{\mu}$ as fundamental degrees of freedom. The zero-modes of these fields, i.e. the small "oscillations" of the world sheet, are the massless fields of the theory, which dominate the effective dynamics at energies below the string scale $1 / \sqrt{\alpha^{\prime}}$. Usually, only they enter into phenomenologically relevant considerations, as this mass scale is conventually assumed to be of the order of the Planck mass. 
The perturbative first quantized formulation of string theory consists of a formalism to compute scattering amplitudes. They have an expansion in powers of the string coupling constant $g_{\mathrm{S}}$ associated to the fundamental string vertex of figure 1.1.

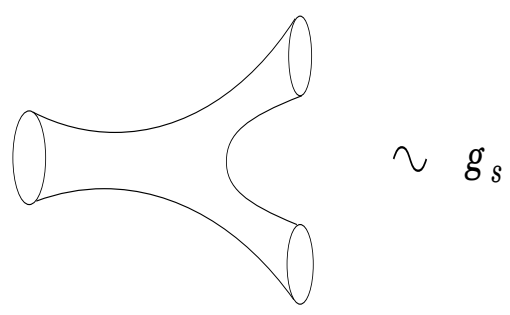

Figure 1.1: The string vertex

The order of perturbation theory is determined by the topology of the world sheet via its Euler characteristic $\chi(\Sigma)$. This perturbative expansion is depicted in figure 1.2 for closed oriented strings, $\chi(\Sigma)$ given by the genus of the surface.

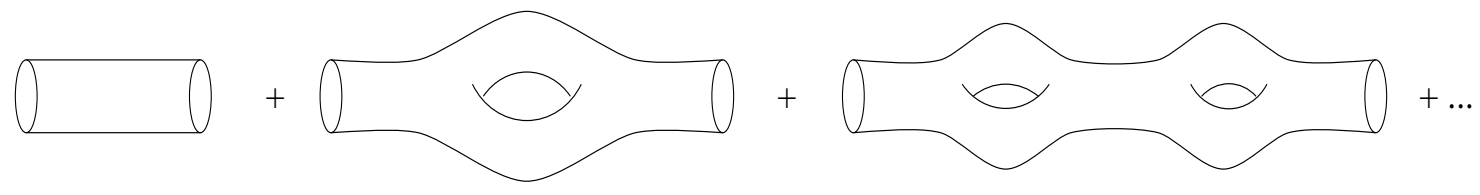

Figure 1.2: Perturbative expansion of string scattering

A crucial condition for the consistency of any superstring theory, related to its unitarity, is that the dimension of the target space is ten. The idea that there may be more than four space-time dimensions in nature, of which some are undetectable to us at laboratory energies, is not original to string theory. It has been addressed for instance in Kaluza-Klein theory already much earlier.

The effective tendimensional field theories which descend from superstring theories by neglecting massive excitations are supergravity theories. The string theories which are usually considered to be fully understood perturbatively lead to 16 or the maximum of 32 supercharges. Furthermore, strings may carry internal degrees of freedom in addition to their coordinates which allow to realize local gauge symmetries. In ten dimensions one is restricted to gauge groups $S O(32)$ or $E_{8} \times E_{8}$. Altogether, string theory does include the major ingredients for supersymmetric extensions of low energy gauge interactions and may serve as a viable unification platform.

\subsection{Compactification}

In a standard compactification of the tendimensional strings one assumes that the space is at least classically of a product structure. One sixdimensional factor is compact and 
usually very small, leaving a noncompact fourdimensional world to live on. Strings which propagate on this space can move along and wrap around the internal space whose size is governed by the compactification scale. Again only the zero-modes of the string fluctuations will be visible at energies below this scale. One is then forced to keep it of the order of the string scale, such that both more or less coincide with the Planck scale itself.

String theory compactifications start from a tendimensional theory with a single kind of interaction and a limited number of massless fields, beside an infinite number of extremely heavy ones. It contains a large amount of supersymmetry, 16 or 32 supercharges corresponding to $\mathcal{N}=4$ or $\mathcal{N}=8$ supersymmetry in four dimensions, and has either very large or no nonabelian gauge groups. The presence of supersymmetry ensures that the known field theoretical scenarios for supersymmetric extensions of the Standard Model can in principle be included in the low energy description of string dynamics. But in order to arrive at fourdimensional effective field theories of any phenomenological relevance a number of points have to be taken care of:

- Supersymmetry has to be broken, which can be done in a number of ways. In order to enjoy the benefits of low energy supersymmetry, it is required that 4 supercharges survive up to the electroweak scale at about $1 \mathrm{TeV}$. If one also wants to maintain the unification of couplings at the GUT scale one has to break the remaining supersymmetries already above the unification scale. One possibility to start with is a breaking of some supersymmetry already at the string or compactification scale and leave four supercharges for the desired $\mathcal{N}=1$ supersymmetry in four dimensions. Actually, we shall also consider a completely alternative "large extra dimension" scenario, which basically identifies the string and electroweak scales and allows to break all supersymmetry at this low scale while still escaping the hierarchy problem.

- The gauge group has to be broken to a suitable subgroup of $S O(32)$ or $E_{8} \times E_{8}$. Again one can devise several opportunities. Instead of a direct reconstruction of the Standard Model gauge group, one may want to have some kind of GUT scenario at an intermediate scale, which only at the electroweak scale breaks down.

- The matter content given by those fields which remain massless at the string or compactification scale has to be suitably adapted to the Standard Model or a desired extension. This implies in particular a spectrum including chiral fermions and scalars with a potential which is suitable to drive a Higgs mechanism.

There are, of course, many special and equally important properties of the Standard Model, which also have to be accounted for, once the more general features are met. It is a very difficult and an unresolved task so far to cover all the issues of constructing realistic low energy models in a single stroke. This will not be achieved here either, but we explore some interesting mechanisms which may be a step towards a more definite answer to be given in the future. 
In mathematical language the requirements above can be related to the properties of the compactification manifold: The requirement to preserve any supersymmetry demands it to be a Kähler manifold of $S U(3)$ holonomy. The surviving supersymmetry is given by those supercharges which are invariant under the holonomy group. The surviving gauge group is the subgroup that is left invariant under the structure group of the gauge bundle over the compactification manifold. The spectrum of massless matter is given by topological invariants which are related to the multiplicities of zero-modes of the equations of motion via index theorems. In this thesis the three issues, the breaking of supersymmetry, the breaking of the gauge group and the generation of chiral fermion spectra will be pursued in a simultaneous approach by using a compactification in exactly solvable backgrounds, tori and toroidal orbifolds together with nonvanishing vacuum expectation values for the internal components of gauge field strengths.

\subsection{Dualities}

In the attempt of viewing the fundamental theory of all forces as the theory of strings, it has for some time been a puzzling fact that string theory in its perturbative definition is not unique. More concretely, there are five perturbatively distinct theories in ten dimensions to be counted, type IIA and type IIB, the heterotic strings with gauge groups $S O(32)$ and $E_{8} \times E_{8}$ as well as the type I string with gauge group $S O(32)$, the only theory with perturbative open strings. The first two have 32 , the latter three 16 supercharges. This picture has changed dramatically when the notion of duality was discovered [4] in string theory. In quantum field theory it means that a given field theory can be transformed into another one by a redefinition of the fields and parameters in the Lagrangian. Formally, this mapping appears to be an identity of theories, but it may involve an inversion of the coupling, such that no meaningful comparison of perturbative results from both sides is possible. Then perturbation theory on the one side by definition allows to compute nonperturbative effects on the other. In string theory such dualities relate all the five theories, involving nonperturbative description of all of them via some dual perturbative model. In particular, after compactification to lower dimensions there are dualities which relate some theory on one space to another theory on a different space. This identification reduces the degeneracy of the string vacuum dramatically as compared to the set of geometrically distinct compactfication manifolds. Finally, the idea has emerged that all formerly distinct string theories join a common moduli space and their perturbative definitions just provide descriptions of different corners. Even more surprising, substantial evidence has been collected that all the theories stem from a still unknown M-theory which itself exists in eleven dimensions.

The evidence on those dualities which invert couplings necessarily relies on results which are exact even beyond perturbation theory, e.g. protected by supersymmetry. On the other hand, there are dualities which are just mappings of one perturbative model to another one. They can be checked perturbatively, and the prototype of such, T-duality, has been proven to be an exact perturbative symmetry of any string theory compactified on tori. It relates tori of different shape and size, an example of a reduction of the vacuum degeneracy. The string moduli space of such compactifications therefore involves a 
quotient by the T-duality group.

\subsection{Open strings and branes}

A concept which is very specific to supergravity and string theory are branes. These are submanifolds of the entire space-time, either wrapped on its compact part or infinitely extended in the noncompact space. They are associated to solitonic solutions of the supergravity equations of motions and often carry conserved topological charges which prevent them from decaying into the vacuum. A particular class of these solutions conserves supersymmetry and simultaneously saturates a certain BPS bound for the minimal energy of the field configuration. These are the stable and extremal BPS branes. The effective field theories of the light fluctuations in such a solitonic background define the world volume theories on the branes. Hence, these are theories whose dynamical degrees of freedom are localized on the respective submanifold of space-time. The properties of the transverse space only enter as parameters into the effective action, such as couplings and masses. A study of the transverse geometry then provides a powerful tool in understanding the evolution of these quantities, i.e. the dynamics of the world volume theory.

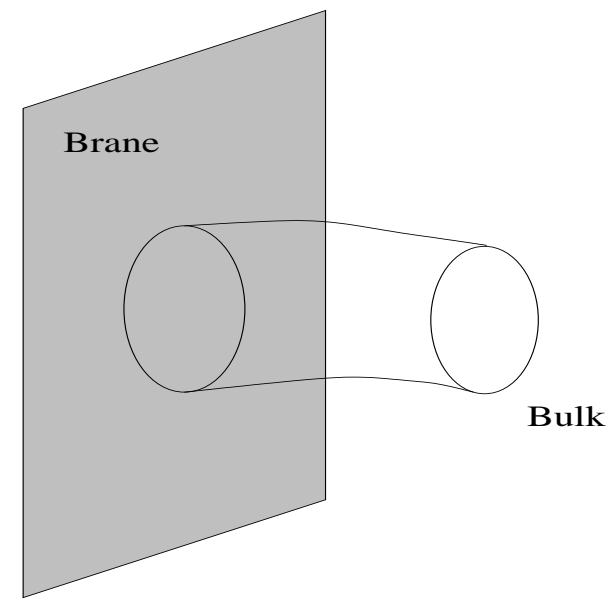

Figure 1.3: A D p-brane

Particularly important examples of branes are the so-called D-branes [5, 6] which can be interpreted in string theory quite naturally. They carry a supersymmetric gauge theory on their world volume $[7,8]$, while the gravitational sector is still tendimensional. In this special case a microscopic description of the fundamental degrees of freedom of the gauge theory is provided by open strings which end on these branes while closed strings still propagate in the bulk and represent the purely gravitational interactions. The coordinates of the world sheet of such a string have Dirichlet boundary conditions along the boundary of the world sheet on the brane, which is why they are termed Dbranes or $\mathrm{D} p$-branes, $p$ indicating the number of spatial dimensions. A novel feature of D-branes is the existence of noncommutativity. In the presence of magnetic flux in the 
gauge theory on its world volume the coordinates of open strings ending on the brane do not commute. When we later employ background gauge fields in order to construct interesting low energy field theories from string compactifications, the geometry of the internal spaces will necessarily turn noncommutative. In fact, these two points of view are equivalent and we could replace the commutative internal space with magnetic field by a noncommutative one without, thus call our approach equally well a compactfication on noncommutative spaces.

At least all the branes which are BPS necessarily carry some conserved charge. The spectrum of string theory and supergravity contains certain antisymmetric tensor fields, $p$-forms, which couple to these charges, either as electric or magnetic sources. A very important consistency requirement for the construction of globally well defined string vacua is then given by the cancellation of such background charges in the noncompact part of space-time. A net background charge signals an inconsistency of the theory at large distances, an anomaly. The amount of charge present can be detected by evaluating appropriate string diagrams. They describe the emission and absorption of such fields from D-branes in the vacuum, being the so-called tadpoles. We shall encounter such conditions eventually under the name of "tadpole cancellation" conditions when dealing with type I models.

\subsection{Outline}

The thesis will be organized as follows: Chapter 2 will start with a fairly general treatment of open strings which propagate in noncompact space-time with magnetic background fields of nontrivial topology. The boundary state of a corresponding D-brane with such a flux on its world volume will provide a world sheet CFT definition of these objects. Therefore, annulus amplitudes can be used to extract geometrical and topological data relevant to open strings in this background. These will include the phenomenologically interesting features of a total or partial breaking of supersymmetry as well as the appearance of chiral fermion spectra in the lower dimensional effective field theories on the D-branes. These will be the ingredients to start a program of type I model building by putting magnetic fields on a simple torus. We shall also point out the relation of the constant magnetic fields to a noncommutative deformation of the coordinate algebra that is defined by open strings ending on the particular D-brane. By a second equivalence this noncommutativity is related to an asymmetric rotation of the open string coordinates, treating left- and right-moving world sheet fields differently. We use this fact to rederive the basic results about noncommutativity obtained from open string theory in background fields by applying such asymmetric rotations in a very direct fashion. Next, we are concerned with the toroidal compactification of D-branes with magnetic background fields, and display a certain T-duality which removes the fluxes and noncommutativity from the brane world volume. This allows a more intuitive derivation and explanation of many features less obvious in the original picture. Finally, we also mention the conceptual and formal modifications when we compactify on toroidal orbifolds.

In chapter 3 we employ the ingredients thus provided to study explicit compactifications of type I strings on noncommutative tori or, equivalently, type I strings with 
additional magnetic background fluxes on the D9-branes. We start with an introduction to tadpole cancellation and its relation to space-time anomalies. This is at the heart of any type I compactfication. As the presence of magnetic background fluxes tends to break supersymmetry, we include a discussion of the basic concept of a "large volume" compactification with low string scale, which may provide a viable playground for at least some of the explicit models discussed in the following. We then present the concrete construction of type I vacua with magnetic background fluxes, their tadpole cancellation conditions and spectra, and show a couple of six- and fourdimensional examples. The method offers a number of phenomenologically attractive features: the breaking of supersymmetry, the reduction of the rank of the gauge group together with chiral fermion spectra and a straightforward way to construct phenomenologically appealing models in four dimensions. A pathology of the original set-up, the absence of odd numbers of fermion generations, can further be cured by including a nontrivial background for the NSNS 2-form as well. The examples we present include a model rather close to the Standard Model, a left-right symmetrically unified model and another GUT scenario. Concerning the compactification of type I with background NSNS 2-form field we also contribute a number of fundamental observations which provide further insight into some of the puzzles related to this discrete modulus. In particular, we show that the general lore about the reduction of the rank of the gauge group does not hold strictly. This is also illustrated by an example.

Finally in chapter 4 we return to the asymmetric rotations equivalent to magnetic background fluxes if applied to open string boundary conditions. We construct type I vacua which are symmetric under these operations, i.e. asymmetric orientifolds. We find a large class of $\mathcal{N}=1$ supersymmetric models in six and four dimensions, which have rather poor phenomenological perspectives but a very exotic internal geometry with different values for the internal magnetic fields identified. They do not distinguish commutative and noncommutative internal spaces. 


\section{Chapter 2}

\section{Open strings on D-branes with background fluxes}

This chapter is rather general in nature. We discuss the propagation of open strings in the presence of nonvanishing constant background values for the magnetic components of the Yang-Mills field strengths. The presentation will not take reference to type I strings and is equally valid for open strings of type II or, upon certain technical modifications, even purely bosonic theories. We first introduce the microscopic world sheet CFT description of open string sectors via boundary states added into closed string theories. For the beginning we simplify to noncompact and flat space-time and show how to include magnetic background fluxes in the boundary conformal field theory. Later we add the necessary modifications for compact toroidal and orbifold spaces. This formalism allows to extract the perturbative dynamics of open strings which propagate in such simple spaces with nontrivial gauge bundles. The chapter is preliminary in preparing the technicalities of type I compactifications with background fluxes which we turn to in chapter 3 , but it is also interesting in its own right to study the conceptual and phenomenological issues of background fluxes at the most generic level. As a pedagogical illustration of the information contained already in the simplest string diagram involved, the annulus, we do some sample calculations in appendix B, where we illustrate how to extract the geometrical data of the gauge bundle on the torus from the world sheet CFT.

The presence of such magnetic background fluxes has a number of phenomenologically interesting consequences [9], a chiral spectrum of fermions in the effective field theory, the breaking of supersymmetry on the world volume of the D-brane as well as a breaking of gauge symmetry which can also include a reduction of the rank of the gauge group. These properties will first be discussed from an effective point of view by analyzing the Yang-Mills theory on D-branes with magnetic background flux. This provides the motivation to start up a program of model building by using internal magnetic fluxes within type I string theory $[10,11,12,13,14]$. The explicit string theoretical construction will actually add numerous aspects to the purely effective approach using the full microscopic definition of open strings on D-branes with flux, not only their low energy approximation.

Another issue that is nowadays being very much appreciated consists in the fact that fluxes on D-branes induce a noncommutative geometry on the world volume of these 
branes, as seen by open string probes $[15,16,17]$. In particular, Seiberg and Witten have shown in [18] that the flux can be absorbed into an effective open string metric $G_{i j}$ and an open string antisymmetric background field $\Theta_{i j}$. This redefinition of fields can again be reexpressed as an asymmetric rotation of string coordinates. We shall point out that the modification of the boundary states which is due to the magnetic field can also be obtained by performing a left-right asymmetric rotation of the boundary conditions of a D-brane without any such flux. Together the identification we present is threefold, fluxes, noncommutative deformations and asymmetric rotations are all different descriptions of the same physical situation [19].

\subsection{Boundary conformal field theory}

To introduce D-branes $[7,5,8,6,20,21]$ into the world sheet CFT of closed strings one adds open string sectors to the Hilbert space, which are built up from certain boundary states $[22,23,24,25,26,27]$. These states satisfy the respective boundary conditions which belong to a particular D-brane. For the free CFT of closed strings on flat spacetime, be it noncompact or a torus, the boundary conditions of interest are easily derived from the world sheet $\sigma$-model of the closed string. They are linearized solutions to the general conditions

$$
\mathcal{T}_{\mathrm{L}}(\tau, \sigma)-\left.\mathcal{T}_{\mathrm{R}}(\tau, \sigma)\right|_{\tau=0}=0
$$

for the left- and right-moving energy momentum tensors at the boundary $\tau=0$.

\subsubsection{Boundary conditions}

In the following we shall frequently refer to the definitions and conventions collected in the appendix A, the closed string coordinates $X^{\mu}(\tau, \sigma)$ and $\Psi^{\mu}(\tau, \sigma)$, their decomposition into left- and right-moving sectors, the Hamiltonians $\mathcal{H}_{\mathrm{op}}$ and $\mathcal{H}_{\mathrm{cl}}$ of open and closed strings etc. We shall always be working in the light-cone gauge where the light-cone components of any physical field have been gauged away. Therefore, we shall not have to deal with reparametrization ghost fields and all component indices refer to space-like directions. For the gauge field strength this implies that we talk about magnetic flux as opposed to electric flux. Starting from any closed string theory, open string sectors are then defined by imposing boundary conditions at $\tau=0$ or $\pi$ on $X^{\mu}$ and $\Psi^{\mu}$, letting $\sigma \in[0,2 \pi)$ be the periodic variable. They need to satisfy the conditions that follow from the $\sigma$-model action

$$
S=\frac{1}{4 \pi} \int_{\Sigma} d \tau d \sigma\left(g_{\mu \nu} \partial_{\alpha} X^{\mu} \partial^{\alpha} X^{\nu}+\epsilon^{\alpha \beta} B_{\mu \nu} \partial_{\alpha} X^{\mu} \partial_{\beta} X^{\nu}\right)-\frac{1}{2 \pi} \int_{\partial \Sigma} d \sigma A_{i} \partial_{\sigma} X^{i}
$$

where we have displayed only the bosonic part and set $\alpha^{\prime}=1$. The fields are the background metric $g_{i j}$, the antisymmetric tensor field $B_{i j}$ from the NSNS sector and the potential $A_{i}$ of the gauge field strength $F_{i j}$ localized at the boundary of the string world sheet $\Sigma$. 
In flat noncompact space-time and in the absence of nontrivial background gauge and tensor fields, $B_{i j}=0=A_{i}$, the only boundary conditions which respect tendimensional Lorentz invariance are pure Neumann

$$
\begin{aligned}
\left.\partial_{\tau} X^{\mu}\right|_{\tau=0} & =\left.\partial_{\tau} \Psi^{\mu}\right|_{\tau=0}=0 \\
p^{\mu} & =\frac{1}{2}\left(p_{\mathrm{L}}^{\mu}+p_{\mathrm{R}}^{\mu}\right)=0 \\
\alpha_{n}^{\mu}+\tilde{\alpha}_{-n}^{\mu} & =\psi_{r}^{\mu}+i \eta \tilde{\psi}_{-r}^{\mu}=0
\end{aligned}
$$

or pure Dirichlet

$$
\begin{aligned}
\left.\partial_{\sigma} X^{\mu}\right|_{\tau=0} & =\left.\partial_{\sigma} \Psi^{\mu}\right|_{\tau=0}=0, \\
w^{\mu} & =\frac{1}{2}\left(p_{\mathrm{L}}^{\mu}-p_{\mathrm{R}}^{\mu}\right)=0, \\
\alpha_{n}^{\mu}-\tilde{\alpha}_{-n}^{\mu} & =\psi_{r}^{\mu}-i \eta \tilde{\psi}_{-r}^{\mu}=0 .
\end{aligned}
$$

The phase factor $\eta= \pm 1$ characterizes the different spin structures along the noncontractible loop at the boundary [28]. Neumann conditions prevent momentum transfer at the boundary whereas Dirichlet conditions fix the coordinates of the boundary to lie in a plane, a D-brane. An open string sector with Dirichlet conditions in $9-p$ spatial directions then defines a $\mathrm{D} p$-brane. A T-duality in some particular direction $\mu$ applied to an open string amounts to swapping Neumann and Dirichlet conditions [5], the $\tau$ and $\sigma$ derivatives on $X^{\mu}$ and $\Psi^{\mu}$, or, equivalently, performing a reflection

$$
(-1,1)^{(i)} X_{\mathrm{L}}^{i}(-1,1)^{(i)}=-X_{\mathrm{L}}^{i}, \quad(-1,1)^{(i)} X_{\mathrm{R}}^{i}(-1,1)^{(i)}=X_{\mathrm{R}}^{i}
$$

of left-moving fields. This is the prototype of an asymmetric operation acting on open strings, which we shall come to study in greater detail and generality.

There are two more variants of boundary conditions which are of any interest in the following, rotated D-branes and D-branes with background flux. The boundary conditions which define the former are trivially obtained by just rotating a D-brane in a twodimensional plane where it is extended in one direction:

$$
\begin{aligned}
& \left.\partial_{\tau}\left(\cos (\varphi) X^{1}-\sin (\varphi) X^{2}\right)\right|_{\tau=0}=0 \\
& \left.\partial_{\sigma}\left(\sin (\varphi) X^{1}+\cos (\varphi) X^{2}\right)\right|_{\tau=0}=0 .
\end{aligned}
$$

The latter are derived from the linear world sheet $\sigma$-model action (2.2) in the presence of a nonvanishing but constant vacuum expectation value for the antisymmetric tensor field. On the disc world sheet the term in the action that involves $B$ can be converted into a boundary term via Stokes' theorem, such that $B_{i j}$ and $\partial_{[i} A_{j]}$ enter the boundary conditions on the same footing

$$
g^{i j} \partial_{\tau} X_{j}+\left.\mathcal{F}^{i j} \partial_{\sigma} X_{j}\right|_{\partial \Sigma}=0
$$

by defining

$$
\mathcal{F}_{i j}=B_{i j}-\partial_{[i} A_{j]}=B_{i j}+F_{i j}
$$


In general one would need to distinguish the bulk 2-form $B$ from the NSNS sector, and the field strength $F$ of the Yang-Mills vector field on the brane. As long as we are dealing with noncompact flat D-branes, we can gauge away the bulk part by a transformation

$$
B^{\prime}=B+d \Lambda, \quad A^{\prime}=A+\Lambda
$$

and are left with a net field $\mathcal{F}$ on the brane. For simplicity we now specialize to the case where we have only $\mathcal{F}_{12}=-\mathcal{F}_{21} \neq 0$ along some D-brane in the 12 directions and a flat gravity background with $g^{i j}=\eta^{i j}$. The boundary conditions then read

$$
\begin{aligned}
\partial_{\tau} X^{1}-\left.\mathcal{F}_{12} \partial_{\sigma} X^{2}\right|_{\tau=0} & =0 \\
\partial_{\tau} X^{2}+\left.\mathcal{F}_{12} \partial_{\sigma} X^{1}\right|_{\tau=0} & =0 .
\end{aligned}
$$

Obviously, the boundary conditions $(2.10)$ and (2.6) are related by a T-duality on $X^{2}$ swapping $\partial_{\tau} X^{2} \leftrightarrow \partial_{\sigma} X^{2}$.

\subsubsection{Boundary states for D-branes with flux}

The states in the generalized closed string Hilbert space, which then fulfill such boundary conditions, are called boundary states $[23,24,26,27]$ and will be denoted by $|\mathrm{D} p\rangle$. The explicit solutions in the harmonic oscillator basis are given by coherent states, eigenstates of the raising and lowering operators $\alpha_{n}$ and $\tilde{\alpha}_{n}$. Strictly speaking, they are not elements of a Hilbert space, one has to pass to a suitable extension, but we shall not worry about this technical problem. The explicit expressions for D-branes with Dirichlet boundary conditions in $i$-directions and Neumann conditions in $\mu$-directions read [29]

$$
\begin{aligned}
|k, \eta\rangle=\exp ( & \sum_{n=1}^{\infty}\left(-\frac{1}{n} \sum_{\mu} \alpha_{-n}^{\mu} \tilde{\alpha}_{-n}^{\mu}+\frac{1}{n} \sum_{i} \alpha_{-n}^{i} \tilde{\alpha}_{-n}^{i}\right) \\
& \left.+i \eta \sum_{r>0}\left(-\sum_{\mu} \psi_{-r}^{\mu} \tilde{\psi}_{-r}^{\mu}+\sum_{i} \psi_{-r}^{i} \tilde{\psi}_{-r}^{i}\right)\right)|k, \eta\rangle^{(0)} .
\end{aligned}
$$

The zero-mode part $|k, \eta\rangle^{(0)}$ will be treated separately. The state decomposes into components for the NSNS and RR sector

$$
|k\rangle^{(0)}=\mathcal{N}\left(|k\rangle_{\mathrm{NSNS}}^{(0)} \pm|k\rangle_{\mathrm{RR}}^{(0)}\right)
$$

with different summation ranges for the oscillator index $r$ in (2.11), of course. For the fermionic zero-modes $\psi_{0}^{\mu}$ and $\tilde{\psi}_{0}^{\mu}$ in the RR sector one defines a basis of lowering and raising operators $\psi_{0}^{\mu} \pm i \psi_{0}^{\mu}$ for their Clifford algebra and chooses the $\eta=-1$ ground state to satisfy

$$
\left(\psi_{0}^{\mu}+i \tilde{\psi}_{0}^{\mu}\right)|k,-\rangle_{\mathrm{RR}}^{(0)}=\left(\psi_{0}^{i}-i \tilde{\psi}_{0}^{i}\right)|k,-\rangle_{\mathrm{RR}}^{(0)}=0
$$

such that the state $|k,+\rangle_{\mathrm{RR}}^{(0)}$ can be obtained by applying raising and lowering operators appropriately. The superpositions which are invariant under the closed string GSO projection

$$
\mathcal{P}_{\mathrm{GSO}}=\frac{1 \pm(-1)^{F_{\mathrm{L}}}}{2} \frac{1+(-1)^{F_{\mathrm{R}}}}{2}
$$


with $F$ denoting the world sheet fermion number, are given by

$$
\begin{aligned}
|k\rangle_{\mathrm{NSNS}}^{(0)} & =\frac{1}{\sqrt{2}}\left(|k,+\rangle_{\mathrm{NSNS}}^{(0)}-|k,-\rangle_{\mathrm{NSNS}}^{(0)}\right), \\
|k\rangle_{\mathrm{RR}}^{(0)} & =\frac{1}{\sqrt{2}}\left(|k,+\rangle_{\mathrm{RR}}^{(0)}+|k,-\rangle_{\mathrm{RR}}^{(0)}\right) .
\end{aligned}
$$

As well, the number $p$ of directions with Dirichlet boundary conditions has to be odd for type IIA and even for type IIB, referring to the sign chosen in (2.14). The normalization and the relative sign of the NSNS and RR components in (2.12) are in principle free. They are fixed in the following way: The spectrum of open strings with both ends on the same D-brane is required to contain a single tendimensional vectormultiplet, which contains eight massless bosonic states. In the annulus diagram these states propagate along the loop providing an overall normalization.
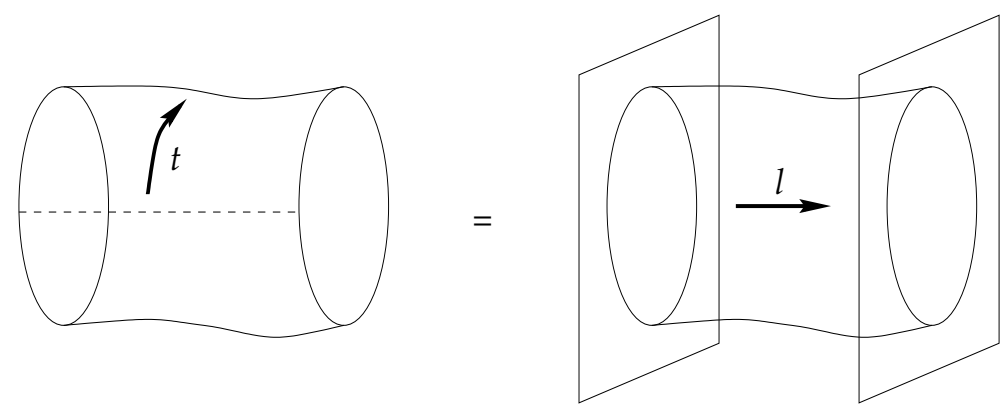

Figure 2.1: Open-closed world sheet consistency

This diagram can be compared to the tree channel exchange of a closed string emitted from the D-brane via a modular transformation, which then fixes the unknown normalization factors for the closed string boundary state. Hence, one requires the so-called open-closed consistency

$$
\int_{0}^{\infty} \frac{d t}{t} \operatorname{Tr}_{\mathrm{NS}-\mathrm{R}}\left(\mathcal{P}_{\mathrm{GSO}} e^{-2 t \mathcal{H}_{\mathrm{op}}}\right)=\int_{0}^{\infty} d l\left\langle\mathrm{D} p\left|e^{-l \mathcal{H}_{\mathrm{cl}}}\right| \mathrm{D} p\right\rangle
$$

of the tree and loop channel annulus amplitude. In appendix B a sample calculation of the simplest case of an annulus diagram is performed, the amplitude of strings stretching between flat D-branes in noncompact space-time, first without and next with additional magnetic fields on their world volume. This also demonstrates how the annulus amplitude depends on the value of the constant magnetic background field and thus captures the geometrical data that describe the gauge bundle. The sign in (2.12) still remains ambiguous, it has no physical effect until branes with different signs for the RR component or additional orientifold planes are considered, when it distinguishes branes a from anti-branes.

The entire boundary state $|\mathrm{D} p\rangle$ is finally defined as a sum over all momentum and winding eigenstates $|k\rangle^{(0)}$, which satisfy the relevant boundary conditions. For a non- 
compact tendimensional space-time it is given by

$$
|\mathrm{D} p\rangle=\prod_{i=p+1}^{9}\left(\int_{0}^{\infty} d k^{i}\right)|k\rangle,
$$

for the complete boundary state $|\mathrm{D} p\rangle$ of a $\mathrm{D} p$ brane extending into the $0 \ldots p$ directions and localized at a point in the transverse space. In compact space the integrations are replaced by summations over the discrete spectrum of zero-modes. These bosonic zeromodes carry the information about the geometry and topology of the space, the D-brane lives on.

In order to obtain boundary states which satisfy the conditions (2.6) for a rotated Dbrane or (2.10) for a D-brane with constant magnetic flux most easily one can use the fact that they are related by a T-duality. Obviously, a rotation in a plane completely along or transverse to the world volume of the brane leaves the coherent state (2.11) invariant. A rotation in some plane where the brane extends in one dimension can be directly implemented by applying the appropriate rotation to the ladder operators. The state which satisfies (2.10) is then given by applying first the rotation and then a T-duality, i.e. a left-moving reflection $(-1,1)^{(i)}$ of the $i$ th coordinate, which swaps Neumann and Dirichlet directions. In order to do this explicitly it is more convenient to complexify coordinates into

$$
Z^{i}=\frac{1}{\sqrt{2}}\left(X^{2 i-1}+i X^{2 i}\right), \quad \bar{Z}^{i}=\frac{1}{\sqrt{2}}\left(X^{2 i-1}-i X^{2 i}\right)
$$

and similarly define the complex raising and lowering operators $\alpha_{n}^{i}$ and $\bar{\alpha}_{n}^{i}, \tilde{\alpha}_{n}^{i}$ and $\tilde{\bar{\alpha}}_{n}^{i}$. In this basis a rotation $\Theta$ acts by

$$
\Theta Z^{i} \Theta^{-1}=e^{i \varphi^{(i)}} Z^{i}, \quad \Theta \bar{Z}^{i} \Theta^{-1}=e^{-i \varphi^{(i)}} \bar{Z}^{i} .
$$

For simplicity take a rotation only within the plane spanned by $x_{1}$ and $x_{2}$ and apply it to a D-brane which extends into $x_{1}$ and is point-like in $x_{2}$. One thus gets the boundary state

$$
\begin{aligned}
\Theta|k, \eta\rangle=|k, \eta\rangle_{(\varphi)} & =\exp \left(-\sum_{n=1}^{\infty} \frac{1}{n}\left(e^{2 i \varphi^{(1)}} \alpha_{-n}^{1} \tilde{\alpha}_{-n}^{1}+e^{-2 i \varphi^{(1)}} \bar{\alpha}_{-n}^{1} \tilde{\bar{\alpha}}_{-n}^{1}\right)\right. \\
+ & \left.\sum_{n=1}^{\infty}\left(-\frac{1}{n} \sum_{\mu \neq 1} \alpha_{-n}^{\mu} \tilde{\alpha}_{-n}^{\mu}+\frac{1}{n} \sum_{i \neq 2} \alpha_{-n}^{i} \tilde{\alpha}_{-n}^{i}\right)\right) \Theta|k, \eta\rangle^{(0)}
\end{aligned}
$$

that satisfies $(2.6)$. The T-duality $(-1,1)^{(2)}$ which produces the boundary state for a D-brane with $\mathcal{F}_{12}=\tan \left(\varphi^{(1)}\right)$ acts as left moving complex conjugation:

$$
(-1,1)^{(2)} \alpha_{n}^{1}(-1,1)^{(2)}=\bar{\alpha}_{n}^{1}, \quad(-1,1)^{(2)} \tilde{\alpha}_{n}^{1}(-1,1)^{(2)}=\tilde{\alpha}_{n}^{1} .
$$

The part for the fermionic oscillators is modified analogously. The action of $\Theta$ on the ground states can most easily be derived in the RR sector, where

$$
\Theta\left|s_{i}\right\rangle_{\mathrm{RR}}=e^{i \sum_{i} s_{i} \varphi^{(i)}}\left|s_{i}\right\rangle_{\mathrm{RR}}=e^{i s_{1} \varphi^{(1)}}\left|s_{i}\right\rangle_{\mathrm{RR}}
$$


the $s_{i}= \pm 1 / 2, i=1, \ldots, 4$, labeling the spinor representation of $S O(8)$. Then a phase factor

$$
\Theta|k\rangle^{(0)}=|k\rangle_{(\varphi)}^{(0)}=\prod_{i}\left(2 \cos \left(\varphi^{(i)}\right)\right)|k\rangle^{(0)}
$$

has to be included accordingly.

\subsection{Chirality and supersymmetry}

In this section we study aspects of the effective theory on D-branes with background fluxes on their world volume, or, equivalently, of D-branes which intersect at angles, especially the breaking of supersymmetry and the appearance of chiral fermions [9].

The supersymmetry that is preserved by a flat $\mathrm{D} p$-brane is defined by the projection operator

$$
\Gamma_{0 \ldots p} \epsilon=\tilde{\epsilon}
$$

$\epsilon$ and $\tilde{\epsilon}$ denoting left- and right-moving generators of supersymmetry transformation. It always leaves exactly one half of the original generators. For branes which are not flat, the condition is still valid locally, written in the tangent frame using vielbeins $e_{\mu}^{a}$,

$$
\Gamma_{\mu}=e_{\mu}^{a} \Gamma_{a},
$$

and imposes restrictions on the embedding of the D-brane into the space-time in order to preserve supersymmetry. These conditions can get modified whenever additional background fields are present.

The massless world volume fields on $N$ parallel D9-branes in noncompact flat space with a trivial gauge bundle combine into the tendimensional $\mathcal{N}=1$ supersymmetric vectormultiplet with eight bosonic and fermionic degrees of freedom in the adjoint representation of a $U(N)$ gauge group. In terms of world sheet oscillators it is given by

$$
A_{\mu} \psi_{-1 / 2}^{\mu} \lambda_{i j}|0\rangle_{\mathrm{NS}}, \quad \chi_{a} \lambda_{i j}|a\rangle_{\mathrm{R}}
$$

The so-called Chan-Paton label $\lambda_{i j}$ is a $N \times N$ matrix which carries the information, on which of the branes the string ends. It transforms in the adjoint representation of the gauge group. The $\mathrm{R}$ ground state is denoted with a spinor index $a$. The open string GSO projection

$$
\mathcal{P}_{\mathrm{GSO}}=\frac{1+(-1)^{F}}{2}
$$

projects to a chiral $U(N)$ gauge theory. On a $\mathrm{D} p$-brane (2.26) needs to be rewritten in terms of $p+1$ dimensional Lorentz indices. When the tendimensional chiral fermion $\chi_{a}$ is reduced to lower dimensions on a space with trivial holonomy, all states survive and a set of nonchiral fermions is obtained. This will stay true for any space where the Dirac 
operator has an equal number of positive and negative chirality zero-modes [30]. Therefore, a simple compactification of D-branes on tori does never lead to phenomenologically interesting field theories in the lower dimensions. This changes when background fields are turned on, to which the fermions couple, even if the space remains flat.

\subsubsection{Supersymmetry breaking}

For D-brane configurations which are supersymmetric otherwise additional magnetic fields can break all or just some supersymmetry. This refers to the theory on the world volume of the branes whereas closed strings which propagate in the bulk are unaffected. Thus, the gravitational sector of the theory will notice the breaking of supersymmetry only via its couplings to the gauge theory sector. This kind of supersymmetry breaking pattern is similar to the "brane supersymmetry breaking" introduced in $[31,32]$. To be explicit first consider the T-dual configuration of two flat $\mathrm{D} p$-branes at relative angles, one of them rotated by $\Theta$ with respect to the first. Preserving any supersymmetry requires [33]

$$
\Gamma_{0 \cdots p} \epsilon=\tilde{\epsilon}, \quad \Gamma_{0 \cdots p} \Theta \epsilon=\Theta \tilde{\epsilon}
$$

which implies

$$
\Theta^{2} \epsilon=\epsilon
$$

We have restricted $\Theta$ to rotate within planes where each of the two branes is extended in precisely one direction. In a suitable complexification (2.18) $\Theta$ can be diagonalized as in $(2.22)$, and the condition

$$
\sum_{i} \pm \varphi^{(i)}=0 \bmod 2 \pi
$$

on the rotation angles $\varphi^{(i)}$ be read off. Therefore, the requirement to have any supersymmetry preserved translates into the condition that $\Theta \in S U(N)$. Whenever the sum of any subset of angles, counted modulo a sign, is vanishing already, even more supersymmetry is preserved, which is in accord with the standard classification via the holonomy of the background geometry: If it is contained in some $S U(N)$ but no $S U(N-1)$ subgroup, any supersymmetry generator in ten dimensions gives rise to only a single generator in $10-2 N$ dimensions.

By a T-duality along all the Dirichlet directions of one of the two D $p$-branes, we get the same relation (2.30) for two D $q$-branes with a background 2-form flux present on one of them. We only have to replace $\mathcal{F}^{(i)}=\tan \left(\varphi^{(i)}\right)$ for the flux in the $i$ th complex plane. The conditions on the angles translate into conditions on the field strength. In four dimensions they just impose its self- or anti-self-duality. It is interesting to note that the condition (2.30) in the "flux" picture derives from a rotation which acts asymmetrically on left- and right-moving fields:

$$
\Gamma_{0 \cdots q} \epsilon=\tilde{\epsilon}, \quad \Gamma_{0 \cdots q} \Theta \epsilon=\Theta^{-1} \tilde{\epsilon} \quad \Rightarrow \quad \Theta^{2} \epsilon=\epsilon .
$$


This is in accord with the results of section 2.3, where magnetic fluxes will be related to asymmetric rotation of open string boundary conditions.

The breaking of supersymmetry is also evident from the variation of the gaugino of the world volume gauge theory on the brane with flux. In addition to the terms originating from the curvature of the space this now includes inhomogeneous terms, which schematically look like

$$
\delta \chi=\mathcal{F}_{i j} \Gamma^{i j} \epsilon+\cdots
$$

From the perspective of the effective theory, their appearance signals a spontaneous breaking of supersymmetry. Formally, the flux has the same effect as a nontrivial holonomy of the background geometry. In other words, for the charged particles not only the geometry of the space, but also that of the gauge bundle is of importance. Such when they travel around any closed path, they do not only feel the curvature but also the flux which runs through the surface which is bounded by that path.

A third presumably equivalent way to classify supersymmetric brane configurations is given by investigating solutions to the "no-force" law of BPS states. The annulus diagram (B.10) of strings between two D-branes with different magnetic fluxes measures such a force. By extracting the massless contributions one finds that they exactly cancel whenever (2.30) holds. By the generalized Jacobi identity (D.4) also the entire amplitude actually vanishes including all the higher string excitations, showing that there are no string theoretical corrections to the low energy classification of supersymmetry.

\subsubsection{Chiral fermions}

When investigating the effective field theory on a D9-brane wrapped on an internal space of real dimension $2 D$ we face a breaking of Lorentz symmetry according to a split

$$
S O(9,1) \longrightarrow S O(d-1,1) \times S O(2 D) .
$$

The spinor representation $\mathbf{1 6}$ in ten dimensions decomposes into lower dimensional representations degenerate under the R-symmetry $S O(2 D)$. For instance, in $d=4$ with $D=3$ one gets

$$
16 \longrightarrow(2,4) \oplus(\overline{2}, \overline{4})
$$

where the $S O(6) \cong S U(4)$ acts on the 4 . If now the holonomy of the internal space is $S U(3) \subset S U(4)$ only a single Majorana-Weil spinor is invariant and the resulting effective theory can have a chiral fermion spectrum. In the same way as the magnetic flux entered the supersymmetry variation of the gaugino, it now affects the massless fermions of the theory by introducing an "effective holonomy" for charged particles. If the rotation, which is associated to the flux by T-duality, is in $S U(N)$ but not in any subgroup $S U(N-1)$, a single fermion in ten dimensions reduces to a single fermion in $10-2 N$ dimensions. In this sense the classification of chirality and supersymmetry go completely hand in hand by looking for covariantly constant spinors, zero-modes of the Dirac operator. 
A direct way to see this is to compute the relevant open string spectrum. Open strings with both ends on the same brane have identical boundary conditions at both ends. Thus, they do not experience the presence of the flux, except for their bosonic zero-mode spectrum, and will produce a nonchiral spectrum. Their mode expansion can be derived from (A.1) and (A.3) by using (2.10) and the same for $\tau=\pi$. The result is (A.7). The world sheet oscillators are still integer moded, while the spectrum of bosonic zero-modes shifts according to the presence of the flux. In the T-dual picture these strings have both ends on a rotated brane. The zero-point energy $E_{0}$ remains unchanged and the vectormultiplet (2.26) massless. Therefore, its reduction does not produce a chiral spectrum in this sector. On the contrary, the sector of open strings stretching between two branes with different fluxes has different boundary conditions at both ends, which projects out the zero-modes and modifies the oscillator expansion and zero-point energy. The solution to the equations of motion with boundary conditions $(2.10)$ with $\mathcal{F}_{1}^{(i)}$ at one end and with $\mathcal{F}_{2}^{(i)}$ at the second end are given by (A.9) [34, 35]. Together with the zero-point energy (A.13) one can then employ the open string Hamiltonian (A.8) to compute the masses of the lightest states in the spectrum. The $\mathrm{R}$ sector ground state always stays massless, but there are no fermionic zero-modes in a plane with relative magnetic flux, such that only one chirality survives in the lower dimensional effective theory. The NS zero-point energy is shifted by

$$
\Delta E_{0}^{\mathrm{NS}}=-\sum_{i} \frac{\delta^{(i)}}{2},
$$

denoting by

$$
\pi \delta^{(i)}=\arctan \left(\mathcal{F}_{2}^{(i)}\right)-\arctan \left(\mathcal{F}_{1}^{(i)}\right)
$$

the relative angle of the T-dual configuration. The lightest states (complex coordinates)

$$
\Phi_{i} \psi_{-1 / 2-\delta^{(i)}}^{i} \lambda_{12}|0\rangle_{\mathrm{NS}}, \quad \bar{\Phi}_{i} \bar{\psi}_{-1 / 2+\delta^{(i)}}^{i} \lambda_{12}|0\rangle_{\mathrm{NS}},
$$

together with those of with orientation, Chan-Paton factor $\lambda_{21}$, have mass squared not necessarily nonnegative. The scalars with negative mass squared pose a problem, but one may try to give a sensible interpretation to their appearance. In principle, these tachyons signal an instability of the vacuum by sitting on a maximum of their potential [36]. By switching on fluxes in more than one complex plane, one can in fact construct configurations where supersymmetry is restored and the tachyons are absent. The conditions which have to be satisfied are given by (2.30). The lightest states in the spectrum of strings stretching between the two branes can also be described by the heuristic formula $[9]$

$$
M^{2}=(2 n+1)|q F|-2 s q F
$$

which is motivated by a quantum mechanical treatment of particles of charge $q$ in a magnetic field $F$. The quantum numbers $n$ and $s$ label the Landau level and the spin of the particle in the directions of the flux. At the lowest level $n=0$ and for $q F>0$ one finds a massless fermion $s=1 / 2$ and a massive state $s=-1 / 2$ together with one scalar 
$s=1$ of negative mass squared and another massive one. The mass spectrum given by (2.38) ensures the vanishing of the supertrace

$$
\operatorname{Str}\left(M^{2}\right)=0,
$$

in accord with a spontaneous breaking of supersymmetry. For the heterotic string, the mass formula can be derived by an exact CFT treatment [37] as an approximation for small values of $F$, whereas in the type I spectrum the states with higher spin $s$ are absent. Therefore there are no tachyons on higher Landau levels as opposed to the heterotic string which has an infinite number of such scalars with negative mass squared. The Landau levels themselves are generated in type I by the lowest bosonic oscillator excitations

$$
\left(\alpha_{\delta^{(i)}}^{i}\right)^{n}|0\rangle
$$

the only oscillator which generates mass splittings proportional to the value $F$ of the field strength. The spectrum of chiral fermions thus obtained is consistent with the geometrical data of the nontrivial gauge bundle. The Dirac operator $\nabla$ measures the number of chiral fermions via its zero-modes of definite chirality. The difference of the numbers of positive and negative chirality sections in an associated spinor bundle over a flat space-time is related to the integral over the Chern character $\operatorname{ch}(\mathcal{F})$ of the gauge bundle via the index theorem of the so-called twisted spin complex [38]

$$
n_{+}-n_{-}=\operatorname{index}(\nabla)=\int_{\mathcal{M}} \operatorname{ch}(\mathcal{F}) .
$$

In the particular case of a $U(1)$ bundle on a twodimensional space the Chern character is given by the first Chern class $c_{1}(\mathcal{F})=\mathcal{F} /(2 \pi)$. In general, it is defined

$$
\operatorname{ch}(\mathcal{F})=\operatorname{Tr} \exp \left(\frac{\mathcal{F}}{2 \pi}\right) .
$$

Therefore, a magnetic background field on $\left(\mathbb{T}^{2}\right)^{3}$ can only give rise to a chiral spectrum, if the corresponding field strength is nontrivial on all three $\mathbb{T}^{2}$. Via T-duality on all $X^{2}$ directions this translates to the statement that the relative angles between the two D-branes must be nonvanishing on all the three $\mathbb{T}^{2}$.

More generally speaking, when we take a stack of $N=M_{1}+M_{2}$ parallel D-branes with relative constant flux between the subsets of $M_{1}$ and $M_{2}$ branes, this breaks the gauge group from $U(N)$ to $U\left(M_{1}\right) \times U\left(M_{2}\right)$. The vectorsupermultiplets in the adjoint of the surviving gauge group stay massless while the rest of the vector fields get masses via a Higgs mechanism. The spectrum also contains massive as well as tachyonic scalars in the bifundamental representation $\left(\mathbf{M}_{1}, \mathbf{M}_{2}\right)$ which only become massless when supersymmetry is preserved. Further, one half of the fermions in the bifundamental representation also remain massless, the other half becoming massive. This bifundamental part of the spectrum of strings stretching between different branes is the source for chiral fermions in a lower dimensional theory, obtained by compactifying on a flat torus but with nontrivial background fluxes along this space. In order to produce chirality in $10-2 D$ dimensions one thus has to introduce fluxes along all $D$ internal planes such that only a single fermion survives the reduction in the $\mathrm{R}$ ground state. Any "trivial" direction would destroy the chirality of the spectrum in the open string sector. 


\subsubsection{Supersymmetric bound states}

As pointed out in the previous section, the sector of open strings stretching between two D-branes with some nonvanishing relative angle $\varphi^{(i)}$ can have excitations of negative mass squared, tachyons. In the effective theory, such a negative mass term signals a maximum of the potential and we would expect a condensation mechanism to take place, leading to a new vacuum with a tachyon condensate. There has been great effort spent in the recent past to understand similar mechanisms of tachyon condensation by employing the formalism of string field theory [39, 40, 41], which is far beyond the scope of this thesis. We shall be content to make plausible that such a mechanism may exist and discuss necessary conditions, which the new vacuum has to satisfy.

In a rather general configuration, two $\mathrm{D} p$-branes at relative angles in $p$ complex planes, there need not necessarily appear a tachyon in the spectrum. As a supersymmetric example, take a D2-D2' configuration characterized by two angles $\varphi^{(1)}$ and $\varphi^{(2)}$, which is T-dual to a D4-D0 setting with magnetic fields $\mathcal{F}_{12}$ and $\mathcal{F}_{34}$ on the D4-brane. The condition to preserve any supersymmetry from (2.30) reads

$$
\varphi^{(1)}+\varphi^{(2)}=\tan \left(\varphi^{(1)}\right)+\tan \left(\varphi^{(2)}\right)=\mathcal{F}_{12}+\mathcal{F}_{34}=0
$$

by choosing the "all plus" convention. Actually, this state is a somehow degenerate example of a $\mathrm{D}(2 p)$-D0 bound state as it is supersymmetric already without any flux or with parallel D2-branes. More general configurations of such states will only become supersymmetric by turning on some flux forming real non threshold bound states $[42,43$, 44]. If the condition (2.43) is violated, there will appear a tachyon in the spectrum which is believed to signal a condensation mechanism related to a deformation of the so far flat cycle which describes the embedding of the D2-D2' system into the fourdimensional space spanned by the 1234 coordinates. For a completely generic embedding

$$
\left(X^{3}, X^{4}\right) \longrightarrow\left(X^{3}+i X^{1}, X^{4}+i X^{2}\right)=\left(Z^{1}, Z^{2}\right)
$$

one obtains the following set of conditions

$$
\frac{\partial X^{1}}{\partial X^{3}}+\frac{\partial X^{2}}{\partial X^{4}}=0, \quad \frac{\partial X^{1}}{\partial X^{4}}-\frac{\partial X^{2}}{\partial X^{3}}=0
$$

In terms of the pull backs of the holomorphic 2-form $\Omega$ and the Kähler form $\omega$

$$
\Omega=d Z^{1} \wedge d Z^{2}, \quad \omega=\frac{1}{2 i}\left(d Z^{1} \wedge d \bar{Z}^{1}+d Z^{2} \wedge d \bar{Z}^{2}\right)
$$

they read

$$
f^{*} \Im(\Omega)=0, \quad f^{*} \omega=0
$$

the well known conditions for a special Lagrangian 2-cycle. The dual interpretation of the configuration is that in addition to the fluxes $\mathcal{F}_{12}$ and $\mathcal{F}_{34}$ there are other components of $\mathcal{F}_{i j}$ turned on. The conditions (2.47) translate to the anti-self-duality condition

$$
\mathcal{F}+* \mathcal{F}=0
$$


of the magnetic field strength, which is the field configuration of a supersymmetric instanton solution to the field equations. These states preserve $1 / 4$ of the initial supersymmetry, just the same as the flat solution (2.43) with nonvanishing relative angles. This analysis can rather straightforwardly be generalized to D3-D3' and D4-D4', respectively D6-D0 and D8-D0, systems [42, 43]. The present methods to study the deformation of supersymmetric cycles by the presence of magnetic fluxes can also be applied to more general cases where the conditions (2.47) get modified [45].

While this is not a strict derivation of the fate of a tachyonic D-brane configuration with magnetic fluxes, it provides us with some confidence to tolerate tachyons in semirealistic model building. Whenever we face tachyons in the spectrum of open strings between two D-branes, we implicitly assume that the tachyon condensation drives the system to a deformed vacuum, where the brane now wraps a general supersymmetric cycle instead of being flat. The massless spectrum and the gauge group of the model should not be affected by this condensation.

\subsection{Noncommutativity and asymmetric rotations}

It has been known already since the works of $[15,16]$ that a background 2-form field on a D-brane is related to a noncommutative deformation of the geometry as being probed by open strings ending on the brane. The recent works $[17,18]$ have put this in a wider context and attracted much attention to noncommutative gauge theories, the effective theories on D-branes with flux. A particular emphasis was put on the existence of a certain map, a redefinition of fields, which transforms the fields of the theory on the commutative space in the presence of a background gauge field into fields on the noncommutative space but without flux, the Seiberg-Witten map of noncommutative gauge theory. While most of the work concerned with these gauge theories naturally deals with the effective Yang-Mills theory, it is also possible to derive the noncommutativity from a microscopic world sheet point of view. We shall pursue this path a little and demonstrate

the following items $[19,10,12]$ : First we review the explicit microscopic calculation of the commutator of open string coordinates on a D-brane with background 2-form flux. We next show the equivalence of the presence of such flux to performing an asymmetric rotation of open string coordinates, which we finally use to rederive the deformation of correlation functions and of the OPE of vertex operators on noncommutative spaces. This establishes a threefold equivalence of background fluxes on D-branes, noncommutativity of their world volume and asymmetric deformations of open string boundary conditions.

\subsubsection{The commutator of open string coordinates}

Let us briefly recall some of the results of the mentioned works in order to set up some notation. From the $\sigma$-model action (2.2) for the disc world sheet one derives equations of motion and the propagator of coordinates, which respects the boundary conditions 
(2.7). In a rather standard fashion one finds [34]

$$
\begin{array}{r}
\left\langle X^{i}(z) X^{j}\left(z^{\prime}\right)\right\rangle=\alpha^{\prime}\left(g^{i j} \log \frac{\left|z-z^{\prime}\right|}{\left|z-\bar{z}^{\prime}\right|}+G^{i j} \log \left|z-\bar{z}^{\prime}\right|^{2}\right. \\
\left.+\frac{\Theta^{i j}}{2 \pi \alpha^{\prime}} \log \frac{z-\bar{z}^{\prime}}{\bar{z}-z^{\prime}}+\text { constant }\right)
\end{array}
$$

using complex world sheet coordinates $z=\tau+i \sigma$. In order to make the $\alpha^{\prime} \rightarrow 0$ limit explicit, we have reintroduced the string tension in this section. The quantities

$$
G=\left(\frac{1}{g+\mathcal{F}}\right)_{\mathrm{S}}, \quad \Theta=2 \pi \alpha^{\prime}\left(\frac{1}{g+\mathcal{F}}\right)_{\mathrm{A}},
$$

subscripts $\mathrm{S}$ and A indicating symmetrization and antisymmetrization, have been introduced in [18] and are called the open string metric $G$ and open string antisymmetric tensor $\Theta .^{1}$ In the flat background, where $g^{i j}=\eta^{i j}$, and for the simple case $\mathcal{F}^{i j}=\mathcal{F} \epsilon^{i j}$ we then have

$$
G^{i j}=\frac{1}{1+\mathcal{F}^{2}} \delta^{i j}, \quad \Theta^{i j}=-\frac{2 \pi \alpha^{\prime} \mathcal{F}}{1+\mathcal{F}^{2}} \epsilon^{i j}
$$

When one specializes the two-point function to the boundary $z=\bar{z}=\tau$ of the world sheet, it is exclusively determined by these two quantities

$$
\left\langle X^{i}(\tau) X^{j}\left(\tau^{\prime}\right)\right\rangle=\alpha^{\prime} G^{i j} \log \left(\tau-\tau^{\prime}\right)^{2}+\frac{i}{2} \Theta^{i j} \operatorname{sign}\left(\tau-\tau^{\prime}\right) .
$$

From this one gets the equal time commutator

$$
\left[X^{i}(\tau), X^{j}(\tau)\right]=i \Theta^{i j}
$$

The effect on the OPE of vertex operators is best exemplified for tachyon states

$$
e^{i p X}(\tau) e^{i q X}\left(\tau^{\prime}\right) \sim\left(\tau-\tau^{\prime}\right)^{2 \alpha^{\prime} G^{i j} p_{i} q_{j}} e^{i(p+q) X}\left(\tau^{\prime}\right)+\cdots
$$

which in the limit $\alpha^{\prime} \rightarrow 0$ reduces to the so-called Moyal-Weil or simply $*$-product

$$
f(x) * g(x)=\left.\exp \left(\frac{i}{2} \Theta^{i j} \frac{\partial}{\partial \zeta^{i}} \frac{\partial}{\partial \xi^{j}}\right) f(x+\zeta) g(x+\xi)\right|_{\xi=\zeta=0},
$$

which is well known in the mathematical literature. In a somewhat simplistic fashion the effect of the $\mathcal{F}$-field for the effective gauge theory may then be summarized by saying that it is equivalent to replace all ordinary multiplications by a $*$-product. Thus a deformation of the coordinate algebra is identical to the physical effect of background 2-form flux.

We now first rederive the above result for the nonvanishing commutator of the coordinates of the boundary of the world sheet of an open string ending on a D-brane with additional flux $\mathcal{F}$ from a microscopic analysis $[46,35]$. This requires the quantization of the bosonic coordinate fields regarding the boundary conditions (2.10). We shall restrict

\footnotetext{
${ }^{1}$ Unfortunately, we use the same symbol $\Theta$ for rotation operators and for the antisymmetric open string tensor.
} 
to the case where the open string has its ends on two D-branes with different fluxes $\mathcal{F}_{1}$ and $\mathcal{F}_{2}$ in some twodimensional plane spanned by $x^{1}$ and $x^{2}$. The solution for this case has already been given in (A.9) in terms of the oscillator expansion. It is to be supplemented with the canonical commutation relations

$$
\left[\alpha_{n+\delta}^{i}, \alpha_{m-\delta}^{j}\right]=(n+\delta) \delta_{n+m, 0} \delta^{i j}
$$

The computation is then a straightforward exercise and reveals

$$
\begin{aligned}
& {\left[X^{1}(\tau, \sigma), X^{2}\left(\tau, \sigma^{\prime}\right)\right]=\left[x^{1}, x^{2}\right]} \\
& +2 i \alpha^{\prime} \sum_{n \in \mathbb{Z}} \frac{1}{n+\delta} \sin \left[(n+\delta) \sigma+\varphi_{1}\right] \sin \left[(n+\delta) \sigma^{\prime}+\varphi_{1}\right],
\end{aligned}
$$

using $\varphi_{\mu}=\arctan \left(\mathcal{F}_{\mu}\right)$. The second term is a representation of the delta function, which has support only when $\sigma=\sigma^{\prime}=0$ or $\pi$. The commutator of the centre of mass coordinates has to be fixed by hand, which is done by requiring that the entire equal time commutator vanishes in the interior of the world sheet

$$
\left.\left[X^{1}(\tau, \sigma), X^{2}\left(\tau, \sigma^{\prime}\right)\right]\right|_{\sigma, \sigma^{\prime} \in \Sigma-\partial \Sigma}=0
$$

which leads to

$$
\left[x^{1}, x^{2}\right]=\frac{2 \pi i \alpha^{\prime}}{\mathcal{F}_{2}-\mathcal{F}_{1}} .
$$

The final result then reads

$$
\left[X^{1}(\tau, 0), X^{2}(\tau, 0)\right]=-\frac{2 \pi i \alpha^{\prime} \mathcal{F}_{1}}{1+\mathcal{F}_{1}}, \quad\left[X^{1}(\tau, \pi), X^{2}(\tau, \pi)\right]=\frac{2 \pi i \alpha^{\prime} \mathcal{F}_{2}}{1+\mathcal{F}_{2}} .
$$

It is evident that the noncommutativity of the D-brane world volume is an entirely local phenomenon. Only the coordinates at the boundary sense the presence of the flux, and each boundary component can have a different deformation of the corresponding coordinate algebra. The total space should then be described as a tensor product of algebras $\mathcal{A}_{\mu}$, one factor for each $\mathrm{D}_{\mu}$-brane present. The generators of any such algebra for a twodimensional space can be taken to be

$$
U_{1}^{\mu}=\exp \left(i x_{1}-\frac{2 \pi^{2} \alpha^{\prime} \mathcal{F}_{\mu}}{1+\mathcal{F}_{\mu}} \frac{\partial}{\partial x_{2}}\right), \quad U_{2}^{\mu}=\exp \left(i x_{2}+\frac{2 \pi^{2} \alpha^{\prime} \mathcal{F}_{\mu}}{1+\mathcal{F}_{\mu}} \frac{\partial}{\partial x_{1}}\right)
$$

satisfying the desired commutator relation

$$
U_{1}^{\mu} U_{2}^{\mu}=U_{2}^{\mu} U_{1}^{\mu} \exp \left(-2 \pi i \frac{2 \pi \alpha^{\prime} \mathcal{F}_{\mu}}{1+\mathcal{F}_{\mu}}\right)
$$

The notion of a unique geometrical description of the space-time as seen by open strings is hence lost somehow in the presence of different background fluxes $\mathcal{F}_{\mu}$ on different $\mathrm{D}_{\mu^{-}}$ branes.

We have already used the T-duality on $X^{2}$ which relates $\mathrm{D} p$-branes with background 2 -form fluxes $\mathcal{F}$ to $\mathrm{D}(p-1)$-branes at relative angles with respect to the direction of the 
T-duality. Let us also look at the commutator of the coordinates of a string stretching between two such D-branes at angles $\varphi_{1}$ and $\varphi_{2}$. The relevant boundary conditions are (2.6) and the mode expansion is given by (A.14). Again one can perform a computation as above to obtain the equal time commutator of coordinates restricted to the boundary of the world sheet, which is found to vanish in all cases:

$$
\left[X^{1}(\tau, \sigma), X^{2}\left(\tau, \sigma^{\prime}\right)\right]=0
$$

Thus the T-duality transforms a commutative space into a noncommutative one when translating angles into fluxes. We shall later employ configurations of D-branes with various fluxes wrapping an internal compact space, a torus. The above analysis equips us with two equivalent descriptions of this geometry, we can call it a noncommutative torus with a variety of sectors with individual deformation of the coordinate algebra, or we use a T-duality to get a selection of intersecting branes of appropriate lower dimensions whose world volume is perfectly commutative. The effective theory of the latter configuration lives on the intersection locus of all the branes. It will be very helpful to employ both pictures, the commutative "angles" picture and the noncommutative "flux" picture simultaneously in order to develop a better intuition of the properties of such settings.

\subsubsection{Asymmetric rotations of D-branes}

Asymmetric operations which treat left- and right-moving world sheet fields differently appear microscopically not to be unnatural, just as the definition of the heterotic string implies a completely different CFT for the two sectors. In terms of asymmetric orbifolds and orientifolds they have been studied since the works of [47]. Still it may be difficult or impossible to give any geometrical interpretation for such operations. A problem arises when one wants to include open strings into the theory, which relate left- and right-moving fields via their boundary conditions. In fact, they equate the left- and right-moving energy momentum currents up to some isometry of the background, which may get in conflict with an arbitrary asymmetric operation even if it were well defined in the closed string sector. In the following we shall study a very particular kind of asymmetric rotations of coordinates, which are shown to be equivalent to turning on background fluxes on the D-branes. We later use these operations to rederive the deformation of the open string correlation functions and the vertex operator $*$-product, as induced by magnetic flux [19].

First recall how a rotation $\Theta$ acts on the complexified world sheet coordinate fields (2.18) of a closed string in some twodimensional plane spanned by $Z^{1}$ and $\bar{Z}^{1}$ via (2.19). A T-duality $T_{2}$ on the imaginary part $X^{2}$ of $Z^{1}$ then acts as a complex conjugation of the left-moving coordinates only

$$
T_{2}\left(Z_{\mathrm{L}}^{1}, Z_{\mathrm{R}}^{1}\right) T_{2}^{-1}=\left(\bar{Z}_{\mathrm{L}}^{1}, Z_{\mathrm{R}}^{1}\right)
$$

Define the action on $\Theta$ by

$$
\hat{\Theta}=T_{2} \Theta T_{2}^{-1}
$$


and combine everything to get

$$
\hat{\Theta}\left(Z_{\mathrm{L}}^{1}, Z_{\mathrm{R}}^{1}\right) \hat{\Theta}^{-1}=\left(e^{-i \varphi} Z_{\mathrm{L}}^{1}, e^{i \varphi} Z_{\mathrm{R}}^{1}\right) .
$$

Hence, the T-duality transforms $\Theta$ into a rotation which treats left- and right-moving fields exactly with opposite phase factors. We have also shown, that the same T-duality translates open string boundary conditions (2.10) for D $p$-branes with flux into boundary conditions (2.6) of $\mathrm{D}(p-1)$-branes at relative angles. Combining the two statements, an asymmetric rotation $\hat{\Theta}$ transforms $\mathrm{D} p$-branes without any flux into $\mathrm{D} p$-branes with such flux, just as the dual rotation $\Theta$ introduces a relative angle with respect to the axis of the T-duality $T_{2}$. Take the boundary conditions for a D-brane which is extended throughout some twodimensional plane

$$
\partial_{\tau} X^{1}=\partial_{\tau} X^{2}=0
$$

decompose into left- and right-moving fields and apply an asymmetric rotation $\hat{\Theta}$ to get

$$
\begin{aligned}
& \partial_{\tau}\left(\cos (\varphi)\left(X_{\mathrm{L}}^{1}+X_{\mathrm{R}}^{1}\right)+\sin (\varphi)\left(-X_{\mathrm{L}}^{2}+X_{\mathrm{R}}^{2}\right)\right)=0 \\
& \partial_{\tau}\left(\cos (\varphi)\left(X_{\mathrm{L}}^{2}+X_{\mathrm{R}}^{2}\right)-\sin (\varphi)\left(-X_{\mathrm{L}}^{1}+X_{\mathrm{R}}^{1}\right)\right)=0 .
\end{aligned}
$$

Finally swap $\partial_{\tau} X_{\mathrm{R}}^{i}=-\partial_{\sigma} X_{\mathrm{R}}^{i}$ to get the boundary conditions (2.10) for a D-brane with background flux $\mathcal{F}_{12}=\tan (\varphi)$. Thus asymmetrically rotated D-branes are identical to such with constant background 2-form flux. We now use this insight to rederive some of the properties of open string coordinates on a noncommutative space.

\subsubsection{Two-point function and OPE}

We first derive the result (2.49) for the propagator of two coordinate fields inserted in the disc diagram from an asymmetric rotation. We start with the propagator in the absence of flux, with Neumann type boundary conditions, which is obtained from (2.49) by setting $\mathcal{F}=0$ and $G^{i j}=\eta^{i j}$. It reads

$$
\begin{aligned}
\left\langle X^{i}(z) X^{j}\left(z^{\prime}\right)\right\rangle & =\alpha^{\prime} \delta^{i j}\left(\log \left|z-z^{\prime}\right|+\log \left|z-\bar{z}^{\prime}\right|\right) \\
& =\alpha^{\prime} \delta^{i j} \frac{1}{2}\left(\log \left(z-z^{\prime}\right)+\log \left(\bar{z}-\bar{z}^{\prime}\right)+\ln \left(z-\bar{z}^{\prime}\right)+\ln \left(\bar{z}-z^{\prime}\right)\right) .
\end{aligned}
$$

One can then formally split the coordinates

$$
X^{i}(z, \bar{z})=X_{\mathrm{L}}^{i}(z)+X_{\mathrm{R}}^{i}(\bar{z})
$$

and apply a left-right-asymmetric rotation $\hat{\Theta}$ treating the four terms in (2.69) individually different. The result is

$$
\begin{aligned}
\left\langle X^{i}(z) X^{j}\left(z^{\prime}\right)\right\rangle=\alpha^{\prime} \delta^{i j}\left(\log \left|z-z^{\prime}\right|+\right. & \left.\left(\cos ^{2}(\varphi)-\sin ^{2}(\varphi)\right) \log \left|z-\bar{z}^{\prime}\right|\right) \\
& +\alpha^{\prime} \epsilon^{i j} \sin (\varphi) \cos (\varphi) \log \left(\frac{z-\bar{z}^{\prime}}{\bar{z}-z}\right) .
\end{aligned}
$$

After identifying $\mathcal{F}_{i j}=\tan (\varphi) \epsilon_{i j}$ this expression agrees precisely with the propagator (2.49). Thus, by applying an asymmetric rotation there is an elegant and short way 
of deriving the propagator in a background 2-form field without explicit reference to the boundary conditions. Moreover, since the commutative D-brane is related in such a smooth way by a continuous rotation to a noncommutative D-brane, one would suspect that there is a map in the effective theory, which maps the fields of the commutative to the noncommutative theory, as was provided by [18].

As a second example we reconstruct the OPE of tachyon vertex operators. Of course, this follows in principle from the propagator (2.49), but we shall directly reproduce it from an asymmetric rotation applied to the standard OPE without any flux. In this way the $*$-product will be found to emerge from this asymmetric operation when acting on open strings. Again we decompose formally the standard OPE of tachyon vertex operators into left- and right-moving contributions

$$
e^{i p X}(z) e^{i q X}\left(z^{\prime}\right)=\frac{\left(z-z^{\prime}\right)^{\alpha^{\prime} p_{\mathrm{L}} q_{\mathrm{L}} / 2}\left(\bar{z}-\bar{z}^{\prime}\right)^{\alpha^{\prime} p_{\mathrm{R}} q_{\mathrm{R}} / 2}}{\left(z-\bar{z}^{\prime}\right)^{\alpha^{\prime} p_{\mathrm{L}} q_{\mathrm{R}} / 2}\left(\bar{z}-z^{\prime}\right)^{\alpha^{\prime} p_{\mathrm{R}} q_{\mathrm{L}} / 2}} e^{i(p+q) X}\left(z^{\prime}\right)+\cdots
$$

restricting to $|z|>\left|z^{\prime}\right|$. Next we apply an asymmetric rotation $\hat{\Theta}$ of the coordinates together with the same asymmetric rotation of the momenta

$$
p_{\mathrm{L}} \rightarrow \Theta p_{\mathrm{L}}, \quad p_{\mathrm{R}} \rightarrow \Theta^{-1} p_{\mathrm{R}}, \quad q_{\mathrm{L}} \rightarrow \Theta q_{\mathrm{L}}, \quad q_{\mathrm{R}} \rightarrow \Theta^{-1} q_{\mathrm{R}}
$$

Finally identify $p_{\mathrm{L}}=p_{\mathrm{R}}$ and $q_{\mathrm{L}}=q_{\mathrm{R}}$ and get

$$
\begin{aligned}
e^{i p X}(z) e^{i q X}\left(z^{\prime}\right)= & \frac{\left|z-z^{\prime}\right|^{\prime} p q / 2}{\left|z-\bar{z}^{\prime}\right|^{\alpha^{\prime}\left(\cos ^{2}(\varphi)-\sin ^{2}(\varphi)\right) p q / 2}} \\
& \times\left(\frac{z-\bar{z}^{\prime}}{\bar{z}-z^{\prime}}\right)^{-\alpha^{\prime} \epsilon^{i j} p_{i} q_{j} \sin (\varphi) \cos (\varphi)} e^{i(p+q) X}\left(z^{\prime}\right)+\cdots .
\end{aligned}
$$

Restricting to the boundary and choosing coventions as in [18] we finally arrive at

$$
\begin{aligned}
e^{i p X}(\tau) e^{i q X}\left(\tau^{\prime}\right)=\left(\tau-\tau^{\prime}\right)^{\alpha^{\prime} p q\left(1+\sin ^{2}(\varphi)-\cos ^{2}(\varphi)\right)} & \\
& \times e^{\left(-i \pi \alpha^{\prime} \sin (\varphi) \cos (\varphi) \epsilon^{i j} p_{i} q_{j}\right)} e^{i(p+q) X}\left(\tau^{\prime}\right)+\cdots
\end{aligned}
$$

This is precisely the OPE derived in $[17,18]$, which shows how one can derive the *product directly via an asymmetric rotation.

\subsection{Toroidal compactification}

So far we have studied the properties of open strings in flat and noncompact space-time in the presence of background 2-form fluxes. All the local properties of string theory only depend on its flatness, such that they carry over to a compactification on a torus. On the contrary, global issues formally encoded in the spectrum of bosonic zero-modes, KK momenta and winding states, will change. 


\subsubsection{Magnetic flux on a torus}

In this section we introduce the basic concept of a toroidal compactification in the presence of magnetic background fluxes. We first go through a number of basic definitions, fix some notation, and then introduce the background 2-form flux into the game. For the reason of simplicity we shall always split our tori into direct factors of twodimensional tori $\mathbb{T}^{2}$,

$$
\mathbb{T}^{2 D}=\bigotimes_{i=1}^{D} \mathbb{T}_{(i)}^{2}
$$

Therefore we can restrict ourselves to a single such $\mathbb{T}^{2}$ for the moment. We choose coordinates for a complex plane as in (2.18) and define a $\mathbb{T}^{2}$ as usual by two identifications

$$
Z \equiv Z+R_{1}, \quad Z \equiv Z+U
$$

imposed on $\mathbb{R}^{2}$, where

$$
U=U_{1}+i U_{2}=\frac{R_{2}}{R_{1}} e^{i \theta} \quad \text { and } \quad T=T_{1}+i T_{2}=b+i R_{1} R_{2} \sin (\theta)
$$

define the complex and the Kähler structures of the torus in terms of the radii $R_{i}$ and the background NSNS 2-form field

$$
B=\frac{b}{R_{1} R_{2} \sin (\theta)}=\frac{b}{T_{2}} .
$$

This defines a twodimensional torus $\mathbb{T}^{2}$ completely. The basis vectors $\mathbf{e}_{i}$ that span the unit cell depicted in figure 2.2

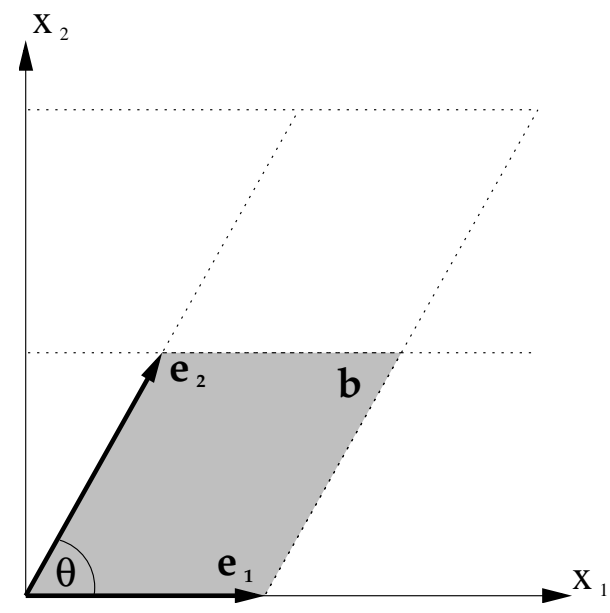

Figure 2.2: The generic torus $\mathbb{T}^{2}$

are given by

$$
\mathbf{e}_{1}=\left(R_{1}, 0\right), \quad \mathbf{e}_{2}=\left(\cos (\theta) R_{2}, \sin (\theta) R_{2}\right)
$$


which can easily be rephrased in terms of $U$ and $T$.

In addition to the background $B$-field of the closed string NSNS sector we can switch on the $U(1)$ field strength $F$ of the Yang-Mills potential $A$. In geometrical terms $A$ is the connection, $F$ the curvature of the $U(1)$ gauge bundle over the torus. This $U(1)$ may in fact be a subgroup of some nonabelian group, such that $F$ is in the Cartan subalgebra of the corresponding Lie algebra. The relevant classification of such $U(1)$ bundles involves three parameters, the first Chern number

$$
C_{1}=\frac{1}{4 \pi} \int_{\mathbb{T}^{2}} F_{i j} d x^{i} \wedge d x^{j}
$$

and the Wilson lines

$$
W_{1}=\int_{\gamma_{1}} A_{i} d x^{i}, \quad W_{2}=\int_{\gamma_{2}} A_{i} d x^{i}
$$

along the two 1-cycles $\gamma_{i}$ of the $\mathbb{T}^{2}$. Physically, $C_{1}$ enters into the Dirac quantization of the field strength, which demands that a suitable Aharanov-Bohm experiment cannot detect any phase factor

$$
\exp \left(2 \pi i n \int_{\mathbb{T}^{2}} F\right)=1
$$

which, for constant field strength $F_{i j}=F \epsilon_{i j}$, reveals

$$
F=\frac{m}{n T_{2}}, \quad \frac{m}{n} \in \mathbb{Q}
$$

The two integers $n$ and $m$ measure the quanta of the electric and magnetic charges. For $\mathcal{F}=B+F$ we get the following quantization condition

$$
\mathcal{F}=B+F=\frac{b}{T_{2}}+\frac{m}{n T_{2}}
$$

It will be of some importance that the Dirac quantization refers to $F$, such that $n T_{2} \mathcal{F}$ may take continuous values in type II and, as we shall show, at least half-integer values in type I string theory. The fact, that there are two integers used to classify the curvature tensor, which geometrically should be fully characterized by $C_{1}$, may look surprising, and will be further elucidated soon. We shall reinterpret these results in terms of the T-dual picture with branes at angles in chapter 2.4.3. In chapter 2.4.4 we shall further demonstrate, how these data enter into the world sheet CFT of open strings ending on these D-branes, more particularly into that part of a boundary state which describes the contributions of the bosonic zero-modes.

\subsubsection{Zero-mode spectra}

In order to obtain a CFT description of D-branes wrapping a torus in the presence of 2 -form flux, we need to supplement the earlier results of section 2.1 with an analysis of the bosonic zero-mode spectra, the KK momenta and winding states. The oscillator part of the Hilbert space represents the local structure and will be unaffected by the 
compactification on a flat torus.

The bosonic zero-modes which respect the periodicity of the torus are conveniently written in the form of left- and right-moving momenta as in (A.2), but combined into complex coordinates in the way of (2.18) and expressed in terms of $U$ and $T$,

$$
\begin{aligned}
& p_{\mathrm{L}}=\frac{1}{i \sqrt{U_{2} T_{2}}}\left(U p_{1}-p_{2}-\bar{T}\left(q_{1}+U q_{2}\right)\right), \\
& p_{\mathrm{R}}=\frac{1}{i \sqrt{U_{2} T_{2}}}\left(U p_{1}-p_{2}-T\left(q_{1}+U q_{2}\right)\right),
\end{aligned}
$$

with integer quantum numbers $p_{i}$ and $q_{i}$. We better use the boundary condition $(2.10)$ in complex form, which is

$$
\begin{aligned}
\partial_{\tau} Z+i \mathcal{F} \partial_{\sigma} Z & =0 \\
\partial_{\tau} \bar{Z}-i \mathcal{F} \partial_{\sigma} \bar{Z} & =0
\end{aligned}
$$

One just has to insert (2.86) into (A.5) and this into (2.87) to find the relations

$$
p_{1}=\left(T_{2} \mathcal{F}\right) p_{2}=\left(b+\frac{m}{n}\right) p_{2}, \quad q_{2}=-\left(T_{2} \mathcal{F}\right) q_{1}=-\left(b+\frac{m}{n}\right) q_{1} .
$$

They have solutions only for rational $b$ and we shall restrict ourselves to this case in the following. Actually, irrational $b$ is somewhat ill defined. In the T-dual picture it implies a brane of infinite volume, densely covering the torus. In any case, there are no bosonic zero-modes on such a brane. For the rational case, we can write $T_{2} \mathcal{F}=r / s \in \mathbb{Q}$ and get an explicit solution

$$
s p_{1}=r p_{2}=p \in r s \mathbb{Z}, \quad r q_{1}=-s q_{2}=q \in r s \mathbb{Z}
$$

for the two sets of quantum numbers. Note that we have to choose $r$ and $s$ co-prime: $(r, s)=(1)$. This produces the mass spectrum

$$
M_{\mathrm{cl}}^{2}=\left|p_{\mathrm{L}}+p_{\mathrm{R}}\right|^{2}+\left|p_{\mathrm{L}}-p_{\mathrm{R}}\right|^{2}=\frac{|p+q U|^{2}}{U_{2}} \frac{|m+n T|^{2}}{T_{2}}
$$

of closed string bosonic zero-mode excitations compatible with the periodicity of the torus and the boundary conditions (2.87). The bosonic ground state of the D-brane boundary state can then be symbolically written in terms of the quantum numbers $p$ and $q$ as

$$
|k\rangle^{(0)}=|p, q\rangle_{(\mathcal{F})}
$$

and depends implicitly on $\mathcal{F}$. The modular transformation of the tree channel annulus diagram into the loop channel of open strings via (D.7) allows to read off the open string mass spectrum as well,

$$
M_{\mathrm{op}}^{2}=\tilde{M}_{\mathrm{cl}}^{2}=\frac{|p+q U|^{2}}{U_{2}} \frac{T_{2}}{|m+n T|^{2}} .
$$

It is in fact not clear how to derive this from a direct quantization of open string momenta. Together with the computations for a noncompact background in appendix B one 
can now easily compute the annulus diagrams of all kinds of strings stretching between D-branes with 2-form background flux on a torus. We shall make excessive use of this in chapter 3 .

As a side remark, we like to provide another example that demonstrates the equivalence of the presence of the background flux and a noncommutative deformation of the geometry by reproducing the mass formula (2.92) from the open string metric and antisymmetric tensor of Seiberg and Witten as given in (2.51). In order to keep the formulas simple we specialize to the case $U_{1}=T_{1}=0$ or $\theta=\pi / 2$. The open string mass formula then simply reads

$$
M_{\mathrm{op}}^{2}=\frac{\left(p R_{1}\right)^{2}+\left(q R_{2}\right)^{2}}{m^{2}+\left(n R_{1} R_{2}\right)^{2}}
$$

and the complexified momentum and winding states are found to be given by

$$
\begin{aligned}
P & =\frac{1}{1+\mathcal{F}^{2}}\left(p_{1}^{\prime}+i p_{2}^{\prime}\right), \\
L & =\frac{\mathcal{F}}{1+\mathcal{F}^{2}}\left(-p_{2}^{\prime}+i p_{1}^{\prime}\right),
\end{aligned}
$$

where we have defined

$$
p_{1}^{\prime}=\frac{p_{1}}{n R_{1}}, \quad p_{2}^{\prime}=\frac{p_{2}}{n R_{2}}, \quad p_{1}, p_{2} \in \mathbb{Z}
$$

The electric charge quantum number $n$ rescales the standard KK momenta along the brane. This suggests to interpret it as the number of times this brane wraps the entire torus $\mathbb{T}^{2}$. In (2.94) the metric $G^{i j}$ of $(2.51)$ defines the deformed momenta and $\Theta^{i j}$ the winding states. The mass formula (2.93) can then also be written

$$
M_{\mathrm{op}}^{2}=|P|^{2}+|L|^{2}=G^{i j} p_{i}^{\prime} p_{j}^{\prime} .
$$

Together we conclude the following: The explicit introduction of the magnetic flux into the boundary conditions produces the same open string KK and winding mass spectrum as does the use of the open string metric and antisymmetric tensor with standard KK momenta, only rescaled by the electric charge. Thus, the equivalence which we have already discussed in chapter 2.3 for the local properties of the D-brane geometry also extends to the global issues.

\subsubsection{T-duality}

In noncompact space-time the T-duality which translates D-branes with magnetic fluxes into D-branes at angles simply swaps the boundary conditions of (2.10) and (2.6) or, equivalently, performs a left-moving reflection of the boundary state in (2.11). Applied to D-branes which are wrapped on a torus it affects the data that describe the background geometry in addition. This operation is actually a particular version of mirror symmetry.

We again specialize to a T-duality on the $X^{2}$ direction, a left-moving reflection of $X^{2}$ or a left-moving complex conjugation of $Z^{1}$. In terms of boundary conditions it 
exchanges the flux $\mathcal{F}_{12}=\mathcal{F}^{(1)}$ which enters in (2.10) with an angle $\varphi^{(1)}$ in (2.6). On the background geometry it acts by an inversion of the radius $R_{2} \rightarrow 1 / R_{2}$, where $\alpha^{\prime}$ is set to 1 . Such the self-dual point in the moduli space, where this operation is a symmetry of the theory is $R_{2}=1$. In terms of the complex and Kähler structures which we use to define a $\mathbb{T}^{2}$ this translates into the exchange of the two

$$
U^{\prime}=-\frac{1}{T}, \quad T^{\prime}=-\frac{1}{U},
$$

where the self-dual point is given by

$$
b=-\frac{R_{1}}{R_{2}^{\prime}} \cos (\theta) .
$$

The dual lattice is spanned by $\mathbf{e}_{i}^{\prime}$, which is depicted in figure 2.3 for $\theta=\pi / 2$ or $b^{\prime}=0$ again.
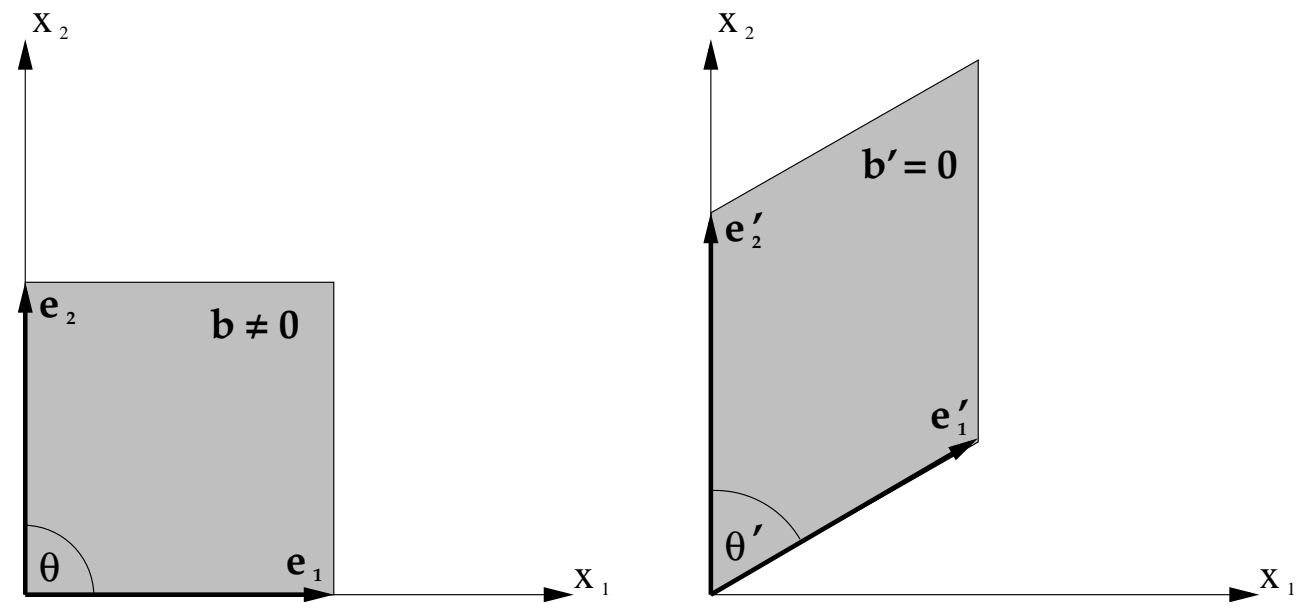

Figure 2.3: T-duality on $X^{2}$ applied to $U$ and $T$

In this case the original torus has a background 2-form flux in the NSNS sector, i.e. $b \neq 0$, while the complex structure is purely imaginary, $U_{1}=0$. Then the T-duality translates to a torus with opposite properties $b^{\prime}=0 \neq U_{1}^{\prime}$. Particularly in type I string this can be very helpful. $U_{1}$ is a continuous modulus of this theory and can be tuned to $U_{1}=0$ without changing the spectrum, the gauge group or other characteristics of the model, while $b$ is discrete and can take only values $b=0,1 / 2$. In this particular situation we will later trade a torus with flux for a tilted torus in order to get a better intuitive understanding of the effects of fluxes in the dual picture.

The former $\mathrm{D} p$-branes which are wrapping the entire torus $n$ times with a gauge bundle of first Chern number given by $m$ translate into $\mathrm{D}(p-1)$-branes which wrap 1-cycles of the torus. If we denote the 1-cycle wrapped by some $\mathrm{D}(p-1)$-brane by

$$
n \mathbf{e}_{1}^{\prime}+m \mathbf{e}_{2}^{\prime} \in H^{1}\left(\mathbb{T}^{\prime 2}\right)
$$


the angle $\varphi$ can be obtained from figure 2.4 by elementary means. It is given by

$$
\tan (\varphi)=\cot \left(\theta^{\prime}\right)+\frac{m}{n T_{2}}
$$

which translates via

$$
\tan (\varphi)=\mathcal{F}_{12}, \quad \cot \left(\theta^{\prime}\right)=B_{12}, \quad \frac{m}{n T_{2}}=F_{12} .
$$

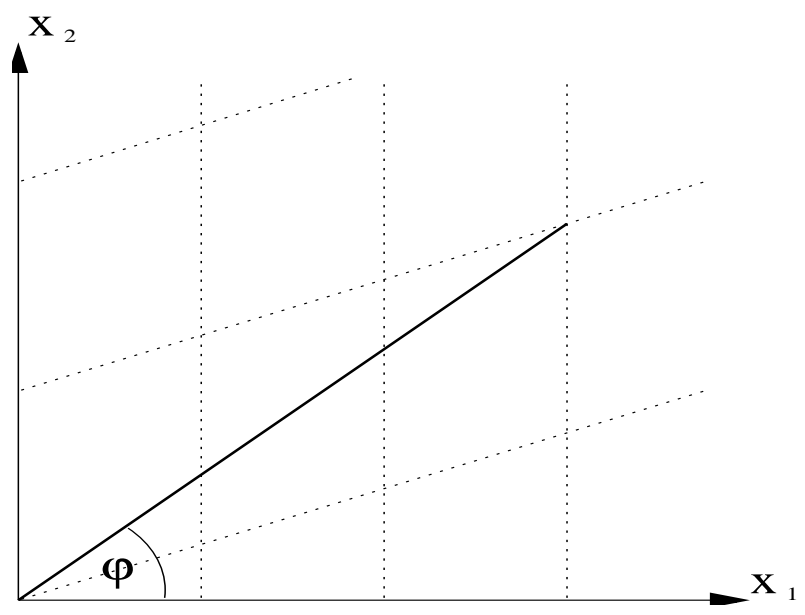

Figure 2.4: An $(n, m)=(3,1)$ D-brane

This relates the wrapping numbers of the $\mathrm{D}(p-1)$-branes on the 1-cycles of the torus to the electric and magnetic charge quantum numbers, i.e. the first Chern number and the wrapping of the $\mathrm{D} p$-brane on the entire torus. It thus exchanges the first and second cohomology groups. The condition for the two charges to take integer values derives from the Dirac charge quantization, whereas the condition that $T_{2} F \in \mathbb{Q}$ derives from the requirement to have a well defined discrete wrapping of the $\mathrm{D}(p-1)$-brane on the torus. It is also clear that $n$ has the interpretation of a total wrapping number in the T-dual "flux" picture: Each intersection of the $\mathrm{D}(p-1)$-brane with the $x_{2}$ axis gives a single copy of this brane under T-duality, such that we are left with a $\mathrm{D} p$-brane wrapping the torus $n$ times.

Some of the ingredients needed to compute amplitudes of open strings stretching between branes wrapping a torus with magnetic flux on their world volume can be obtained very easily in the T-dual "angles" picture. As long as $b^{\prime}=0$, i.e. $U_{1}=0$, the spectrum of open string KK and winding states can literally be written down. The open string KK momenta $P^{\prime}$ along the D-brane are simply the standard ones

$$
P^{\prime}=\frac{p}{V^{\prime}}
$$

on brane of length

$$
V^{\prime}=\sqrt{\left(n R_{1}^{\prime}\right)^{2}+\left(m R_{2}^{\prime} \sin \left(\theta^{\prime}\right)\right)^{2}} .
$$


Similarly, the winding states $L^{\prime}$ are given by the distance

$$
D^{\prime}=\frac{R_{1}^{\prime} R_{2}^{\prime} \sin \left(\theta^{\prime}\right)}{\sqrt{\left(n R_{1}^{\prime}\right)^{2}+\left(m R_{2}^{\prime} \sin \left(\theta^{\prime}\right)\right)^{2}}}=\frac{T_{2}^{\prime}}{V^{\prime}}
$$

to the next copy of the brane in the elementary cell of the torus,

$$
L^{\prime}=q D^{\prime}
$$

The open string mass spectrum is then simply given by

$$
M_{\mathrm{op}}^{\prime 2}={P^{\prime 2}}^{2}+L^{\prime 2}
$$

which perfectly coincides with (2.93) after setting $\theta=\pi / 2$ and $R_{2} \rightarrow 1 / R_{2}$. Thus we can reproduce a moderately tedious computation in the CFT of open strings with magnetic flux by a very straightforward and simple geometrical interpretation in the T-dual picture with D-branes at angles.

A second input to open string diagrams of strings stretching between branes at different angles is the presence if extra multiplicities from multiple intersections of any two branes on the torus. The number of times two $\mathrm{D}(p-1)$-branes, labeled by indices $\mu$ and $\nu$ and characterized by wrapping numbers $\left(n_{\mu}, m_{\mu}\right)$ and $\left(n_{\nu}, m_{\nu}\right)$, intersect on any given $\mathbb{T}^{2}$ can be derived by simple topological arguments to be

$$
I_{\mu \nu}=m_{\mu} n_{\nu}-n_{\mu} m_{\nu}
$$

where the sign refers to the orientation. An example is shown in figure 2.5.

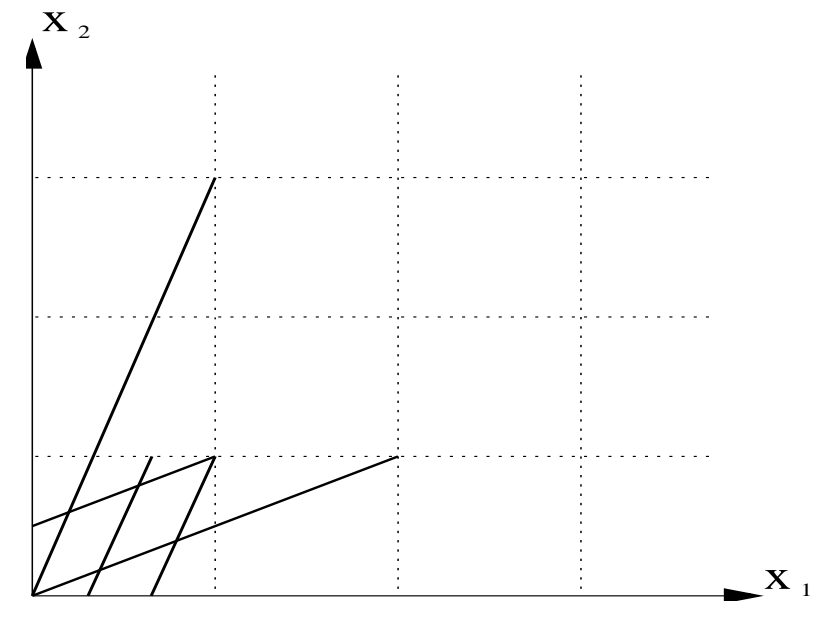

Figure 2.5: Intersecting $(1,2)$ and $(3,1)$ D-branes

This extra multiplicity of the open string sector appears in the trace of the loop channel annulus amplitude and can thus be computed via purely field theoretical methods, which shall be demonstrated in the next chapter. 


\subsubsection{Topological data from CFT}

We have already seen how the world sheet CFT which describes the propagation of open strings on tori with background fluxes encodes the geometrical data of the gauge bundle. Concretely, the first Chern class of the bundle enters via the zero-mode spectrum into the boundary state of the particular D-brane. The Wilson lines are rather trivial to implement, they introduce a shifting of KK momenta. In the T-dual "angles" picture these data have a more intuitive interpretation: The first Chern class is related to the relative angle of the D-brane with respect to the axis of the T-duality. In fact, one can perform another T-duality orthogonal to the world volume directions of the $\mathrm{D}(p-1)$-brane, which produces a $\mathrm{D} p$-brane but with $\mathcal{F}=0$, i.e. vanishing first Chern class. Thus, T-duality can be used to trivialize the gauge bundle on any single D-brane. Of course, there is no further control about the background geometry during this second duality, the moduli of the torus can be completely generic afterwards. In the T-dual picture one of the Wilson lines has the interpretation of shifting the distance between the brane and its next copy on the torus, the second remains a Wilson line along the $\mathrm{D}(p-1)$-brane. In the special "gauge" when the Chern number is dualized to zero they are the only two parameters to classify inequivalent gauge fields. In particular, any noncommutative gauge theory can be T-dualized to become commutative on a different torus and with generic Wilson lines turned on. This simple picture gets mixed up a little, when the T-dual torus still has $b \neq 0$.

As mentioned at the end of the previous section, the T-duality also introduces the notion of extra multiplicities in the open string annulus amplitude, given by the intersection numbers (2.107) of any two branes. These multiplicities are essential to obtain anomaly-free spectra for the low energy effective theories, such they cannot be ignored. In the following we show how these extra factors can also be found by a pure CFT computation in the original "flux" picture without referring to possibly misleading geometrical intuition. In principle most of the technical steps needed to do the computation are performed in appendix B. One first has to compute the tree channel annulus diagram $\tilde{\mathcal{A}}_{\mu \mu}$ of strings with both ends on the same $\mathrm{D}_{\mu}$-brane, read off the normalization of the boundary state by demanding the correct asymptotics after the modular transformation to the loop channel, and afterwards use this information to compute the diagram $\mathcal{A}_{\mu \nu}$ for the strings with ends on different D-branes. There the extra prefactor for the degeneracy of the spectrum due to multiple intersections shows up in the loop channel.

Technically, the only difference as compared to appendix B will be the spectrum of bosonic zero-modes, which is now discrete and depends on the flux $\mathcal{F}_{\mu}^{(i)}$ on the $\mathrm{D} p_{\mu}$-brane on the torus $\mathbb{T}_{(i)}^{2}$. The generic expression for the closed string mass spectrum is (2.90),

but we can turn off $U_{1}^{(i)}=T_{1}^{(i)}=0$ for simplicity again. One can also check directly that this does not affect the outcome. We will not go through the details of the computations but only sketch the modifications as compared to the noncompact case which is treated in appendix B. Also we specialize to a single $\mathbb{T}^{2}$ with nonvanishing fluxes $\mathcal{F}_{\mu}$ which breaks supersymmetry but is perfectly sufficient as an example. The contribution of the 
zero-modes to the integrand of $\tilde{\mathcal{A}}_{\mu \mu}$ then reads schematically

$$
\tilde{\mathcal{A}}_{\mu \mu} \sim \mathcal{N}_{\mu}^{2} \sum_{p, q} e^{-\pi l \tilde{M}_{\mu}^{2}}
$$

with $\tilde{M}_{\mu}^{2}$ given by $(2.90)$ using $\mathcal{F}_{\mu}$, i.e.

$$
\tilde{M}_{\mu}^{2}=\left(\frac{q^{2}}{R_{1}^{2}}+\frac{p^{2}}{R_{2}^{2}}\right)\left(m^{2}+\left(n R_{1} R_{2}\right)^{2}\right)
$$

where $V_{\mu}$ is defined in (2.103). The modular transformation into the tree channel is performed by using the Poisson formula (D.7). It produces a prefactor which needs to be canceled by the normalization $\mathcal{N}_{\mu}$ of the boundary state in order to have the correct multiplicity of massless states in the loop,

$$
\mathcal{N}_{\mu}^{2} \sim \frac{m^{2}+\left(n R_{1} R_{2}\right)^{2}}{R_{1} R_{2}}
$$

The tree channel amplitude $\tilde{\mathcal{A}}_{\mu \nu}$ of open strings with mixed boundary conditions then has a prefactor $\mathcal{N}_{\mu} \mathcal{N}_{\nu}$, but there is also the overall phase factor (2.23). When the dust settles, the relevant part of the loop channel integrand has the schematic form

$$
\mathcal{A}_{\mu \nu} \sim \mathcal{N}_{\mu} \mathcal{N}_{\nu} \sin (\pi \delta) \sum_{p, q} e^{-2 \pi t M_{\mu}^{2}}
$$

and one finds that

$$
\mathcal{N}_{\mu} \mathcal{N}_{\nu} \sin (\pi \delta)=I_{\mu \nu}
$$

with $I_{\mu \nu}$ as defined in (2.107) and $\delta$ by (2.32). Such the normalization of the boundary state, which is basically dictated by the bosonic zero-mode spectrum, encodes the extra multiplicity in the open string spectrum of strings stretching between the two different D-branes. Geometrically, it is obvious only in the T-dual picture where it comes as an intersection number. This may be seen as an example where the CFT on the string world sheet via its boundary conditions detects topological data of the background space-time, be it even rather trivial data. In particular, the extra multiplicities may easily escape a purely field theoretical approach.

For theories which have more structure than the simple compactification of a bosonic or type II brane on a flat torus the above concept may be generalized. In type II theories a topological index

$$
\operatorname{Tr}_{\mathrm{R}}(-1)^{F} \sim \mathrm{RR}\left\langle \pm\left|e^{-l \mathcal{H}_{\mathrm{cl}}}\right| \mp\right\rangle_{\mathrm{RR}}
$$

has been proposed, related to the so-called Witten index [48]. It counts the "intersections" of boundary states just as chiral fermions. In type I theory the world sheet parity $\Omega$ can have a nontrivial action on the boundary states, in particular on those with nonvanishing flux. The theory then also contains orientifold planes in addition to D-branes, and their normalization in a similar fashion encodes the properties of the intersections with respect to their mapping under $\Omega$. While naively $\Omega$ does not operate geometrically 
on the coordinates, the CFT computation shows that not all of the intersections of any two D-branes with magnetic flux are invariant, such that the spectrum in the invariant sectors may differ from those which come in pairs. This is again essential in obtaining consistent low energy theories. The same argument applies to the action of isometries of the background geometry being gauged in orbifold constructions which we turn to next. There the twisted components of the boundary states allow to deduce the projections in the various open string sectors, i.e. the mapping of intersections of D-branes under the respective isometry.

\subsection{Orbifold compactification}

We have shown how flat background spaces with nontrivial gauge bundles produce a number of features that usually arise only from curved background. The picture can be enriched by combining the two effects, take nontrivial geometry together with nontrivial gauge bundles. Of course, this generalized approach leads to an even greater variety of possibilities for the construction of interesting vacua, phenomenologically as well as conceptually speaking. Unfortunately, there are only very few examples of spaces with curvature that still allow a microscopic world sheet description.

We shall restrict ourselves to the case of toroidal orbifold compactifications [49, 50], which, as a first step, may be characterized as quotient spaces of tori. One uses complexified tori defined by the factorization $(2.76)$ into $\mathbb{T}_{(i)}^{2}$ each given by its complex and Kähler structures, $U^{(i)}$ and $T^{(i)}$, and identifies points under some discrete group of isometries of the torus. Outside the fixed points of this group, the resulting spaces are still flat, such that all the nontrivial effects are concentrated in these singularities. Transporting a field along a closed loop around one of them, it may be periodic only up to the action of a group element. The most general operation of the group on a section in the tangent bundle of a torus of complex dimension $D$ is the maximal $S O(2 D)$ holonomy. In order to preserve some supersymmetry one has to restrict to $S U(D)$ subgroups, which implies the space to be Kähler and Ricci-flat. The resulting manifolds are then particular singular limits of smooth complex manifolds, of the K3 for $D=2$ and of Calabi-Yau manifolds for $D=3$.

We will not be very much concerned with the more mathematical aspects of orbifolds and adopt a more heuristic point of view. Defining a closed string theory on such spaces

is a pretty straightforward thing to do. One first has to restrict to the equivariant part of the original theory on the torus, i.e. one has to project the closed string Hilbert space to the invariant subspace. We shall only consider abelian isometry groups given by a single cyclic factor $\mathbb{Z}_{N}=\left\{1, \Theta, \ldots, \Theta^{N-1}\right\}$. The generator $\Theta$ is simply a rotation of coordinates,

$$
\Theta Z^{i} \Theta^{-1}=e^{i \varphi^{(i)}} Z^{i}
$$

which may be accompanied with an action on the gauge bundle, denoted by unitary matrices $\gamma_{\Theta \mu}$,

$$
\lambda_{i j}^{\mu} \stackrel{\Theta}{\longrightarrow}\left(\gamma_{\Theta \mu}\right)_{i i^{\prime}} \lambda_{i^{\prime} j^{\prime}}^{\mu}\left(\gamma_{\Theta \mu}^{-1}\right)_{j^{\prime} j},
$$


acting on the Chan-Paton labels. The projector which defines the so-called untwisted sector of the orbifold Hilbert space is then defined by

$$
\mathcal{P}_{\text {orb }}=\frac{1+\Theta+\cdots+\Theta^{N-1}}{N} .
$$

Some properties of such projections have already been studied in the previous chapters. Supersymmetry is preserved precisely if (2.30) holds and the spectrum of the effective lower dimensional theory may contain chiral fermions. By imposing the requirements of preserving some amount of supersymmetry and not getting any tachyonic closed string ground states in the twisted sectors, to be explained below, one can set up a table of acceptable rotations $\Theta[50]$. If we denote $\Theta$ by its eigenvalues $v_{i}$ in terms of $S U(D)$ generators $J_{i}$,

$$
\Theta=\exp \left(2 \pi i \sum_{i=1}^{D} v_{i} J_{i}\right),
$$

one has the following supersymmetric orbifolds of K3

\begin{tabular}{|ll|ll|}
\hline Group & $\left(v_{1}, v_{2}\right)$ & Group & $\left(v_{1}, v_{2}\right)$ \\
\hline \hline $\mathbb{Z}_{2}$ & $(1 / 2,-1 / 2)$ & $\mathbb{Z}_{4}$ & $(1 / 4,-1 / 4)$ \\
$\mathbb{Z}_{3}$ & $(1 / 3,-1 / 3)$ & $\mathbb{Z}_{6}$ & $(1 / 6,-1 / 6)$ \\
\hline
\end{tabular}

Table 2.1: K3 orbifolds

The choice of signs for the $v_{i}$ is conventional, according to the condition (2.30) for preserving supersymmetry. Of course, the moduli of the tori must be tuned in a way to allow a crystallographic action of $\Theta$, which is the reason that no larger cyclic groups are present. The table for supersymmetric orbifolds of threedimensional Calabi-Yau spaces reads

\begin{tabular}{|ll|ll|ll|}
\hline Group & $\left(v_{1}, v_{2}, v_{3}\right)$ & Group & $\left(v_{1}, v_{2}, v_{3}\right)$ & Group & $\left(v_{1}, v_{2}, v_{3}\right)$ \\
\hline \hline $\mathbb{Z}_{3}$ & $(1 / 3,1 / 3,-2 / 3)$ & $\mathbb{Z}_{6}^{\prime}$ & $(1 / 6,2 / 6,-3 / 6)$ & $\mathbb{Z}_{8}$ & $(1 / 8,3 / 8,-4 / 8)$ \\
$\mathbb{Z}_{4}$ & $(1 / 4,1 / 4,-2 / 4)$ & $\mathbb{Z}_{7}$ & $(1 / 7,2 / 7,-3 / 7)$ & $\mathbb{Z}_{12}$ & $(1 / 12,4 / 12,-5 / 12)$ \\
$\mathbb{Z}_{6}$ & $(1 / 6,1 / 6,-2 / 6)$ & $\mathbb{Z}_{8}$ & $(1 / 8,2 / 8,-3 / 8)$ & $\mathbb{Z}_{12}^{\prime}$ & $(1 / 12,5 / 12,-6 / 12)$ \\
\hline
\end{tabular}

Table 2.2: Calabi-Yau orbifolds

In addition to the states invariant under the isometry $\Theta$ there may appear twisted fields in the orbifold theory Hilbert space, which do not stem from the projection. They have $m$-twisted periodicity

$$
Z^{i}(\tau, \sigma+2 \pi)=\Theta^{m} Z^{i}(\tau, \sigma) \Theta^{-m}=e^{i m \varphi^{(i)}} Z^{i}(\tau, \sigma) .
$$


Due to

$$
z_{0}^{i}=e^{i m \varphi^{(i)}} z_{0}^{i}, \quad \bar{z}_{0}^{i}=e^{-i m \varphi^{(i)}} \bar{z}_{0}^{i}
$$

they are localized at some of the fixed points of the orbifold space. When resolving the singularities, a mathematically well defined procedure that allows a transition to a smooth manifold, one discovers a matching of these new twisted fields and the moduli which describe the geometry of the resolution [51]. More concretely, but still roughly, one cuts out the singularity and pastes in some number of $\mathbb{P}^{n}$ 's. They replace the vanishing cycles given by the fixed points and, for example, need to be accounted for in determining the correct cohomology of the space. The parameters which characterize the $\mathbb{P}^{n}$ 's are called twisted moduli. In this sense one says that the CFT of the string world sheet already resolves the fixed points at the geometrically singular point of the moduli space. Actually, further difficulties are connected to these singularities, such as the problem of a quantum of NSNS $B$-flux which is believed to be hidden in the vanishing cycles.

The mode expansion of a twisted coordinate field is given by solving the equations of motion subject to the periodicity conditions (2.118), which gives (A.15). An obvious feature is the shifting of the Fourier modings which we have already encountered with open string coordinates for mixed boundary conditions, be it those stretching between branes with different flux, or those between branes at relative angles. The zero-point energy of the orbifold sector obeys the same analogy, it is given by the formula (A.13) applied with the appropriate shift of modings. Finally, the closed string Hamiltonian of the orbifold theory is (A.16), identical to (A.8) only replacing $n$ by $n \pm m \delta^{(i)}$ and $r$ by $r \pm m \delta^{(i)}$.

Open string sectors are introduced into orbifold theories in a way completely analogous to the standard way. Since the twisted sector fields are stuck at the fixed points of the orbifold group anyway, one can only have twisted boundary states which are strictly localized inside this locus. The boundary conditions for such branes are still given by (2.4). Written in terms of complex coordinates (A.15) they become

$$
\begin{aligned}
& \alpha_{n+m \delta^{\prime}}-\tilde{\alpha}_{-n-m \delta^{\prime}}=0, \\
& \bar{\alpha}_{n-m \delta^{\prime}}-\tilde{\bar{\alpha}}_{-n+m \delta^{\prime}}=0,
\end{aligned}
$$

with similar expressions for the fermionic fields. See for instance [52] for a more complete treatment. For simplicity we here focus on a single complex plane, which, of course, would not be a supersymmetric orbifold, but is perfectly suitable to explain the technical steps. From the boundary conditions one can easily construct the coherent state in the $m$ twisted sector,

$$
\begin{aligned}
|k, \eta, m\rangle=\exp \left(\sum_{n=1}^{\infty}(\right. & -\frac{1}{n} \sum_{\mu} \alpha_{-n}^{\mu} \tilde{\alpha}_{-n}^{\mu}+\frac{1}{n-m \delta^{\prime}} \alpha_{-n+m \delta^{\prime}} \tilde{\bar{\alpha}}_{-n+m \delta^{\prime}} \\
& \left.\left.+\frac{1}{n+m \delta^{\prime}} \bar{\alpha}_{-n-m \delta^{\prime}} \tilde{\alpha}_{-n-m \delta^{\prime}}\right)\right)|k, \eta, m\rangle^{(0)}
\end{aligned}
$$

for convenience, with Neumann boundary conditions in the transverse eightdimensional space-time. Again we have only displayed the bosonic part. For the fermionic state one 
also needs to regard that there are no fermionic zero-modes in the $\mathrm{R}$ ground states along the orbifold. The bosonic momentum ground state is defined by an integration over momenta only in the transverse directions as well. This defines the complete boundary state $|\mathrm{D} p, m\rangle$ of the $m$-twisted sector. The most general boundary state in an orbifold theory is then given by a superposition of untwisted and twisted components invariant under the orbifold projection,

$$
\left|\mathrm{D} p^{\text {orb }}\right\rangle=\mathcal{P}_{\text {orb }} \sum_{m=0}^{N-1} \mathcal{N}_{m}|\mathrm{D} p, m\rangle .
$$

The relative normalization factors need to be chosen in order to satisfy the open-closed consistency as discussed already for the boundary state of a D-brane with flux. Concretely, this implies that the contribution of the $m$-twisted component to the tree channel annulus amplitude transforms to the insertion of $\Theta^{m}$ in the trace of the loop channel expression. The correct normalization of the projector in the loop channel thus fixes the ambiguity in determining the $\mathcal{N}_{m}$. We shall use this requirement as a technical tool in chapter 4 in order to construct consistent orbifold vacua of type I or type $\mathrm{I}^{\prime}$. In practice, we prefer to compute the relevant amplitudes in the loop channel, such we do not need to specify the normalization here explicitly. In fact, it can be easily determined from the loop channel expressions which we shall calculate. 


\section{Chapter 3}

\section{Type I superstrings with background fluxes}

As an introduction we first review the basic issues of type I string theory. We show how it emerges as a truncation of the type IIB superstring, how the problem of quantum corrections to the vacuum in the form of tadpoles arises and how it is cured in tendimensional type I string theory in a unique fashion by introducing D9-branes which support the gauge fields of the effective field theory. We next point out a particularity of type I theory, the opportunity of large volume compactifications with a low string scale of the order of the electroweak scale. Only then we proceed to our main subject the compactification of type I on tori and orbifolds in the presence of nontrivial magnetic background fields.

Type I string theory is the unique tendimensional string theory where open strings appear at the perturbative level. It is defined primarily by performing a truncation of the type IIB theory which has identical left- and right-moving world sheet sectors. One can then define type I string theory by projecting onto states invariant under the world sheet parity $\Omega[53,54,55,5]$. It exchanges the left- and right-moving fields,

$$
\Omega X_{\mathrm{L}}^{\mu} \Omega^{-1}=X_{\mathrm{R}}^{\mu}, \quad \Omega \Psi_{\mathrm{L}}^{\mu} \Omega^{-1}=\Psi_{\mathrm{R}}^{\mu}
$$

by mapping $\sigma \rightarrow \sigma^{\prime}=2 \pi-\sigma$. The massless bosonic spectrum of type IIB in ten dimensions consists of the metric, the NSNS 2-form $B$ and the dilaton,

$$
\begin{gathered}
G_{\mu \nu}\left(\psi_{-1 / 2}^{(\mu} \tilde{\psi}_{-1 / 2}^{\nu)}-\eta_{\rho \sigma} \psi_{-1 / 2}^{\rho} \tilde{\psi}_{-1 / 2}^{\sigma}\right)|0\rangle_{\mathrm{NSNS}} \\
B_{\mu \nu} \psi_{-1 / 2}^{[\mu} \tilde{\psi}_{-1 / 2}^{\nu]}|0\rangle_{\mathrm{NSNS}} \\
\Phi \eta_{\mu \nu} \psi_{-1 / 2}^{\mu} \tilde{\psi}_{-1 / 2}^{\nu}|0\rangle_{\mathrm{NSNS}}
\end{gathered}
$$

and a set of tensor fields from the RR sector, a 4 -form $C^{(4)}$, a 2 -form $C^{(2)}$ and the $\mathrm{RR}$ scalar $C^{(0)}$,

$$
\begin{gathered}
C_{\mu \nu \rho \sigma} \chi_{a}^{T} \Gamma^{\mu \nu \rho \sigma} \chi_{\tilde{a}}\left(|a\rangle_{\mathrm{R}} \otimes|\tilde{a}\rangle_{\mathrm{R}}\right) \\
C_{\mu \nu} \chi_{a}^{T} \Gamma^{\mu \nu} \chi_{\tilde{a}}\left(|a\rangle_{\mathrm{R}} \otimes|\tilde{a}\rangle_{\mathrm{R}}\right) \\
C \chi_{a}^{T} \chi_{\tilde{a}}\left(|a\rangle_{\mathrm{R}} \otimes|\tilde{a}\rangle_{\mathrm{R}}\right)
\end{gathered}
$$


In a more compact notation this set of fields is usually written

$$
\begin{aligned}
\left(\mathbf{8}_{V} \oplus \mathbf{8}_{S}\right) \otimes\left(\mathbf{8}_{V} \oplus \mathbf{8}_{S}\right)=(\mathbf{3 5} \oplus \mathbf{2 8} \oplus \mathbf{1})_{\mathrm{NSNS}} & \\
& \oplus(\mathbf{3 5} \oplus \mathbf{2 8} \oplus \mathbf{1})_{\mathrm{RR}} \oplus \text { fermions }
\end{aligned}
$$

the tensor product of the left- and right-moving fields in terms of $S O(8)$ representations. Now $\Omega$ reflects the NSNS 2-form and the RR 0 - and 4-forms, such that the surviving spectrum is a graviton plus dilaton and an extra 2 -form tensor field.

The crucial observation which leads to the introduction of open strings, carrying the gauge field degrees of freedom is the requirement of the cancellation of one-loop tadpole diagrams in order to have a consistent theory. The philosophy and techniques of such tadpoles and their relation to space-time anomalies will be discussed in the following section. In fact, it is mandatory to have a background of 32 D9-branes which fill out the entire space-time. Such D-branes carry a charge under the RR fields, in particular under a 10-form potential $C^{(10)}[6]$, which neutralizes a uniformly distributed background charge induced at the one-loop level by a nonorientable closed string diagram, the Klein bottle. The resulting vacuum is neutral and stable. In principle, D-branes which support the open string sectors can also be introduced in type II string theory, but whenever they fill out the noncompact space-time they have to be accompanied by anti-branes of opposite charge in order to guarantee the global consistency of the theory. This in turn introduces an instability, as such brane-anti-brane pairs tend to annihilate.

\subsection{Tadpole cancellation and anomalies}

The most important requirement in the construction of type I string theory is the cancellation of tadpoles. While it has lead to very powerful tools and recipes in constructing consistent open string vacua, it still bears a bit of a mystery. From the point of view of the effective field theory in the noncompact part of space-time the string theoretical tadpoles are interpreted as background sources. It appears that a string vacuum that is free of tadpoles does not suffer from any kind of space-time anomaly, such that tadpole cancellation is a very powerful condition summarizing a variety of restrictions on the spectra of lower dimensional effective theories.

\subsubsection{Nonorientable string diagrams}

At the level $\chi=2-2 g-b-c=0$ of the first orientable closed string loop diagram, the torus, there are three additional diagrams, two of them nonorientable, two with boundaries, the Klein bottle, the annulus and the Möbius strip. Starting from type IIB theory one first has to investigate the effect of inserting the projector

$$
\mathcal{P}_{\Omega}=\frac{1+\Omega}{2}
$$

into the torus diagram $\mathcal{T}$. The latter itself is given by the Coleman-Weinberg formula

$$
\mathcal{T}=\int \frac{d^{2} \tau}{\Im(\tau)} \operatorname{Tr}\left(\frac{1}{2} \mathcal{P}_{\mathrm{GSO}} e^{2 \pi i\left(\tau L_{0}-\bar{\tau} \bar{L}_{0}\right)}\right) .
$$


The integral over the complex modulus $\tau$ contains the conformally invariant measure, and a fundamental domain for $\tau$ does not include the origin. The trace is over the entire closed string Hilbert space of oscillator excitations and zero-modes, $L_{0}$ and $\bar{L}_{0}$ are the left- and right-moving parts of the Hamiltonian as defined in (A.6). As the massive excitations are exponentially suppressed, the only potential divergencies could stem from the massless states. But due to the fact that $\tau_{2}>0$ also their contribution to the integral stays finite. The oscillator part of the torus partition function factorizes into left- and right- moving parts such that the result is essentially given by the square of the trace in the annulus diagram (B.5).

\section{Klein bottle tadpoles}

The second term of (3.5), the insertion of $\Omega$ into the trace projects onto the subspace of left-right symmetrical string excitations. One also has to regard, that $\Omega$ identifies points on the torus world sheet via $\sigma \equiv 2 \pi-\sigma$, which has been depicted in the third diagram of figure 3.1 .
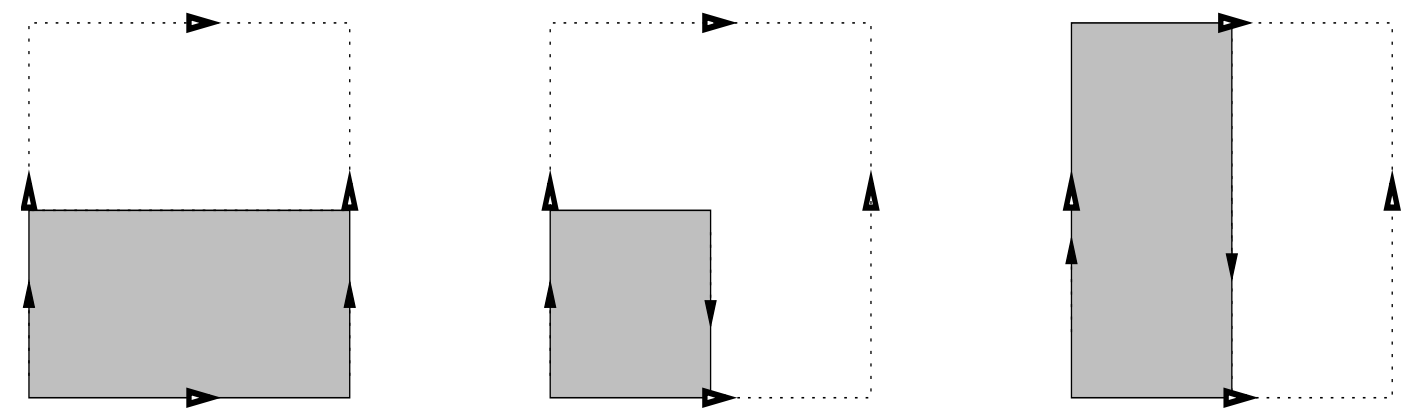

Figure 3.1: Fundamental domains for the annulus, Möbius strip and the Klein bottle

Effectively, the world sheet is no longer a torus but is projected to the nonorientable world sheet at Euler characteristic $\chi=0$, the Klein bottle. It has an equivalent tree channel description as a sphere with two cross-cap insertions. The figure 3.1 illustrates the embedding of all three world sheets of the open and nonorientable closed string diagrams with $\chi=0$, the annulus, Möbius strip and Klein bottle into the torus diagram. The correct modular transformation for the annulus diagram, the first one shown, maps $l$, the "length" of the tree level channel world sheet, into $1 /(2 t)$, that of the loop channel one, the factor 2 being an arbitrary normalization. For the Klein bottle, the third one, filling one half of the fundamental domain of the torus due to the $\Omega$ projection the transformation exchanges $l$ and $1 /(4 t)$. For the Möbius strip, the second one, $l$ is mapped to $1 /(8 t)$.

Concerning the Klein bottle amplitude, the $\Omega$ projection thus also changes the moduli space of conformally inequivalent world sheets. While tori are parameterized by a complex modulus $\tau$, the inequivalent Klein bottles have a real parameter $t$ related to $\Im(\tau)$ but now ranging over all positive numbers. The amplitude then reads in its loop 
channel

$$
\mathcal{K}=\int_{0}^{\infty} \frac{d t}{t} \operatorname{Tr}\left(\frac{\Omega}{2} \mathcal{P}_{\mathrm{GSO}} e^{-2 \pi t\left(L_{0}+\bar{L}_{0}\right)}\right) .
$$

The GSO projection has no effect since the $\Omega$ projection enforces equal fermion numbers for left- and right-movers anyway. The result for the integrand is formally given by the "diagonal part" of the torus diagram,

$$
\mathcal{K}=2^{-2} c \int_{0}^{\infty} \frac{d t}{t^{6}} \sum_{\alpha, \beta \in\{0,1 / 2\}}(-1)^{2(\alpha+\beta)} \frac{\vartheta\left[\begin{array}{l}
\alpha \\
\beta
\end{array}\right]^{4}}{\eta^{12}}
$$

where the integration over momenta has been summarized in $c$ as defined in (B.7). The $\vartheta$-functions, with argument $q=\exp (-4 \pi t)$, encode all the oscillator contributions. In order to compare this diagram to closed string diagrams with boundaries, tree level transition functions of boundary states, as in (B.3), it is necessary to perform a modular transformation that takes the Klein bottle loop diagram into a transition function of two orientifold boundary states, formally provided by the modular transformation $l=1 /(4 t)$. It makes use of the formulas (D.6) for the transformation properties of the $\vartheta$-functions and leads to

$$
\tilde{\mathcal{K}}=2^{4} c \int_{0}^{\infty} d l \sum_{\alpha, \beta \in\{0,1 / 2\}}(-1)^{2(\alpha+\beta)} \frac{\vartheta\left[\begin{array}{l}
\alpha \\
\beta
\end{array}\right]^{4}}{\eta^{12}}=2^{4} c(1-1) \int_{0}^{\infty} d l \frac{\vartheta\left[\begin{array}{c}
1 / 2 \\
0
\end{array}\right]^{4}}{\eta^{12}},
$$

now with argument $\tilde{q}=\exp (-4 \pi l)$. The prefactor $(1-1)$ refers to the cancellation of NSNS and RR fields which signals the no-force law, also valid for orientifold planes. As the integration over moduli includes the origin, there is a potential divergence for massless fields. Indeed, the expansion of the $\vartheta$-functions reveals

$$
\tilde{\mathcal{K}}=2^{4} c(1-1) \int_{0}^{\infty} d l\left(2^{4}+o\left(e^{-\pi l}\right)\right),
$$

a divergence, caused by the massless fields of the respective sectors. The cancellation of the divergencies via supersymmetry is not sufficient to ensure the global consistency of the theory. The reason is the following: Given that there is a term in the effective Lagrangian which involves the integral of a RR tensor field $C^{(10)}$ of rank 10 over the entire tendimensional space-time,

$$
\mathcal{S}_{\text {eff }}^{(10)}=\mu_{10} \int_{\mathbb{R}^{d}} C_{1 \cdots 10}^{(10)} d x^{1} \wedge \ldots \wedge d x^{10} .
$$

Its equation of motion only reads $\mu_{10}=0$. In other words, if a field enters the Lagrangian only linearly, there cannot occur an extremum of the action except if the coefficient vanishes, i.e. the field is a Lagrange multiplier for $\mu_{10}$. The tadpole contribution of the Klein bottle to the RR sector now implies a quantum correction to the vacuum expectation value of this 10 -form field. In this sense an orientifold plane functions as a background charge for the RR form that couples to its world volume, the entire space-time. Since this is the only coupling of $\mathrm{RR}$ forms which respects tendimensional Lorentz invariance, 
it must be the origin of the divergency in the RR contribution to the Klein bottle. We shall point out its relation to space-time anomalies in the next section, which leads to the conclusion that any RR tadpole implies a severe inconsistency of the space-time effective field theory, and thus needs to be canceled already at the string level. In fact, the same coupling also occurs for D9-branes, such that one can remove the inconsistence by adding additional D9-branes into the background. This will add open string sectors into the theory, strings which end on these branes and contribute at the same $\chi=0$ level via the orientable annulus diagram and the nonorientable Möbius strip.

The second divergent contribution with opposite sign, the NSNS tadpole, is believed to be not as severe than the RR tadpole. In the NSNS sector the field which is responsible for the divergent contribution is the dilaton $\Phi$. The divergence is then called the dilaton tadpole. In contrast to the RR 10-form it appears with a kinetic term and as an overall factor in the action, schematically

$$
\mathcal{S}_{\text {eff }}^{(\Phi)}=\int_{\mathbb{R}^{d}} \sqrt{-G} e^{-2 \Phi}\left(R+4(\partial \Phi)^{2}+\cdots\right) .
$$

A source term, the loop-correction present in the Klein bottle or the tension of a D-brane, can in principle be incorporated into the equations of motion, but will lead to a spacedependent background value for the dilaton, breaking tendimensional Lorentz invariance. This is usually called the Fischler-Susskind mechanism [56, 57], which refers to the first studies of tadpoles in purely bosonic string theory. It is not very much known about the existence and stability of such solutions for the type I superstring. The few examples which have been studied in detail exhibit interesting features $[58,59]$. It appears that the source term for the dilaton enforces a spontaneous compactification of space-time, in this sense providing a dynamical explanation for compactification.

To summarize, the absence of any tadpole, be it RR or NSNS, provides us with a check of all consistency requirements known so far for type I compactifications, while we may want to tolerate NSNS tadpoles on heuristic grounds. Of course, as long as we have unbroken supersymmetry, the vanishing of the entire one-loop amplitude links the vanishing of the $R R$ tadpole to that of the dilaton tadpole. We next go through some more details of the concrete calculation which is involved with the computation of the additional open string contributions to the one-loop amplitude.

\section{Open string diagrams}

The annulus contribution has already been computed in (B.3). It has a similar expansion as the Klein bottle,

$$
\tilde{\mathcal{A}}=2^{-6} N^{2} c(1-1) \int_{0}^{\infty} d l\left(2^{4}+o\left(e^{-\pi l}\right)\right) .
$$

$N$ D9-branes contribute $N$ units of RR charge to the background. The final contribution from the Möbius strip comes with the insertion of the world sheet parity into the trace over open string states. Its action on open strings is defined by mapping $\sigma$ to $\pi-\sigma$, 
where $\sigma \in[0, \pi]$, as opposed to the closed string coordinate $\sigma \in[0,2 \pi)$. It thus acts by a phase factor

$$
\Omega \psi_{r}^{\mu} \Omega^{-1}=e^{-i \pi r} \psi_{r}^{\mu}
$$

on fermionic world sheet oscillators. Formally the result for the loop channel amplitude

$$
\mathcal{M}= \pm \int_{0}^{\infty} \frac{d t}{t} \operatorname{Tr}\left(\frac{\Omega}{2} \mathcal{P}_{\mathrm{GSO}} e^{-2 t \mathcal{H}_{\mathrm{op}}}\right)
$$

can be written very similarly as compared to the annulus amplitude (B.5) by using a modified argument $q=-\exp (-2 \pi t)$ for the $\vartheta$ - and $\eta$-functions,

$$
\mathcal{M}= \pm 2^{-7} N c(1-1) \int_{0}^{\infty} \frac{d t}{t^{6}} \frac{\vartheta\left[\begin{array}{c}
1 / 2 \\
0
\end{array}\right]^{4}}{\eta^{12}} .
$$

This can again be transformed into the tree channel with $l=1 /(8 t)$. This produces

$$
\tilde{\mathcal{M}}= \pm N c(1-1) \int_{0}^{\infty} d l \frac{\vartheta\left[\begin{array}{c}
1 / 2 \\
0
\end{array}\right]^{4}}{\eta^{12}}
$$

with argument $\tilde{q}=-\exp (-4 \pi l)$. Its expansion is

$$
\tilde{\mathcal{M}}= \pm N c(1-1) \int_{0}^{\infty} d l\left(2^{4}+o\left(e^{-\pi l}\right)\right)
$$

Together the three terms, Klein bottle, annulus and Möbius strip, add up to a perfect square

$$
\tilde{\mathcal{K}}+\tilde{\mathcal{A}}+\tilde{\mathcal{M}}=2^{-6} c(1-1) \int_{0}^{\infty} d l\left(2^{4}(N \pm 32)^{2}+o\left(e^{-\pi l}\right)\right) .
$$

Thus the tadpoles cancel if we choose the minus sign for the contribution of the Möbius strip and take $N=32$ D9-branes. In type II theory 32 D9-branes would support a $U(32)$ gauge group on their world volume, the massless fields given by the vectormultiplet $(2.26)$ with $\lambda_{i j}$ in the adjoint of $U(32)$. But in type I the action of $\Omega$ on the oscillator part of the gauge boson

$$
\begin{aligned}
\Omega \psi_{-1 / 2}^{\mu} \lambda_{i j}|0, i j\rangle_{\mathrm{NS}} & =-\psi_{-1 / 2}^{\mu}\left(\gamma_{\Omega}\right)_{i i^{\prime}} \lambda_{i^{\prime} j^{\prime}}\left(\gamma_{\Omega}^{-1}\right)_{j^{\prime} j}|0, j i\rangle_{\mathrm{NS}} \\
& =-\psi_{-1 / 2}^{\mu}\left(\gamma_{\Omega}\right)_{j i^{\prime}} \lambda_{i^{\prime} j^{\prime}}\left(\gamma_{\Omega}^{-1}\right)_{j^{\prime} i}|0, i j\rangle_{\mathrm{NS}}
\end{aligned}
$$

introduces a sign, referring to the minus chosen for the contribution of the Möbius strip amplitude. It needs to be canceled by an appropriate operation on the Chan-Paton index, denoted by $\gamma_{\Omega}$, a unitary matrix, in order to keep the gauge boson in the spectrum. If we choose a flat gauge bundle, i.e. a trivial representation for $\Omega, \gamma_{\Omega}=1$, the Chan-Paton indices need to be antisymmetric, $\lambda_{i j}=-\lambda_{j i}$ and the gauge group is only $S O(32)$, the antisymmetric subset of the set of unitary matrices. 


\section{Orientifold planes}

It has proven very useful to reinterpret the loop diagrams in terms of tree level transition functions, which we have already done formally by using the proper modular transformations. One can construct boundary states $|\mathrm{O} 9\rangle$ for the cross-caps as well, called orientifold 9- or simply O9-planes, and then obtain the entire tree channel one-loop amplitude directly as [23, 26, 27, 25]

$$
\tilde{\mathcal{K}}+\tilde{\mathcal{A}}+\tilde{\mathcal{M}}=\int_{0}^{\infty} d l\left(\left\langle\mathrm{D} 9|N+\langle\mathrm{O} 9|) e^{-l \mathcal{H}_{\mathrm{cl}}}(|\mathrm{O} 9\rangle+N|\mathrm{D} 9\rangle) .\right.\right.
$$

This amplitude thus measures the couplings of the closed string fields to the charges carried by the orientifold plane and the D-branes.

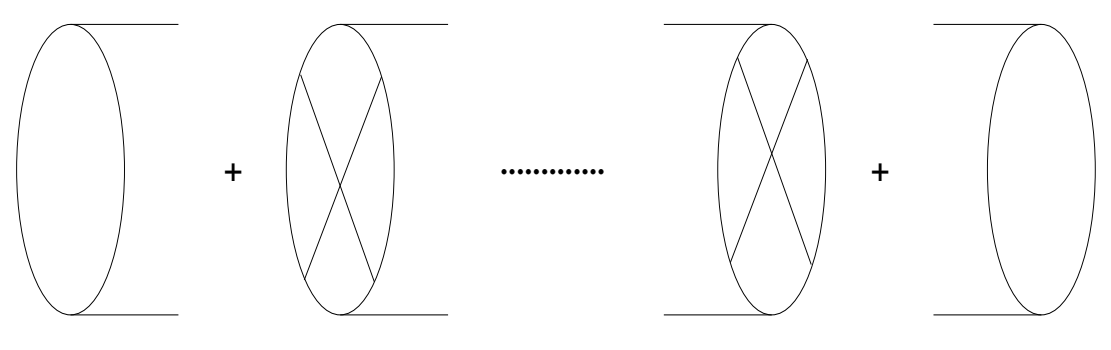

Figure 3.2: Tree channel one-loop amplitude in type I

The orientifold $\mathrm{O} p$-planes $|\mathrm{O} p\rangle$ are defined by changing the boundary conditions of the boundary states (2.11) by phase factors. The $\Omega$ projection identifies $\sigma \equiv \sigma^{\prime}=2 \pi-\sigma$, such that the boundary conditions for a cross-cap are

$$
\left.\partial_{\tau} X^{\mu}\right|_{\tau=0, \sigma}+\left.\partial_{\tau} X^{\mu}\right|_{\tau=0, \sigma^{\prime}}=0
$$

as compared to pure Neumann boundary conditions (2.3). In terms of oscillators this reads

$$
\alpha_{n}^{\mu}+e^{i \pi n} \tilde{\alpha}_{-n}^{\mu}=\psi_{r}^{\mu}+i \eta e^{i \pi r} \tilde{\psi}_{-r}^{\mu}=0 .
$$

In compact space one also has to evaluate the spectrum of bosonic zero-modes which are left invariant under $\Omega$. In the absence of background flux, it leaves KK momenta invariant and reflects winding states. The coherent state $|\mathrm{O} p, k, \eta\rangle$ which defines the oscillator part of the cross-cap state $|\mathrm{O} p\rangle$ is now a simple modification of (2.11) by appropriate phase factors,

$$
\begin{aligned}
|\mathrm{O} p, k, \eta\rangle=\exp ( & \sum_{n=1}^{\infty} e^{i \pi n}\left(-\frac{1}{n} \sum_{\mu} \alpha_{-n}^{\mu} \tilde{\alpha}_{-n}^{\mu}+\frac{1}{n} \sum_{i} \alpha_{-n}^{i} \tilde{\alpha}_{-n}^{i}\right) \\
& \left.+i \eta \sum_{r>o} e^{i \pi r}\left(-\sum_{\mu} \psi_{-r}^{\mu} \tilde{\psi}_{-r}^{\mu}+\sum_{i} \psi_{-r}^{i} \tilde{\psi}_{-r}^{i}\right)\right)|\mathrm{O} p, k, \eta\rangle^{(0)}
\end{aligned}
$$

One can now proceed in the straightforward fashion to compute all the amplitudes as done in chapter 2.1 for the annulus. By computing the tree channel Klein bottle and annulus 
amplitudes one can normalize the respective orientifold plane and boundary states by looking at the loop channel expression that follows from the modular transformation. One gets unambiguous results for the Möbius strip and any diagram of open strings with ends on different D-branes. This procedure of constructing type I vacua can sometimes become a tedious task. While for the heterotic string basically the only requirement of consistency is the modular invariance of the partition function, determined by the level-matching of states, we shall have to compute the entire one-loop amplitude.

\subsubsection{Space-time anomalies}

It is a kind of common knowledge that a vanishing $\mathrm{RR}$ tadpole in the one-loop amplitude of the type I string implies the cancellation of all irreducible anomalies in the effective field theory in the target space. It somehow summarizes the irreducible contributions to the gravitational, nonabelian and the mixed anomalies leaving only reducible contributions which need to be canceled by a suitably generalized Green-Schwarz mechanism. In quantum field theory anomalies signal a breakdown of classical symmetries at the quantum level,

$$
\partial^{\mu} j_{\mu}^{a}=\partial^{\mu} \frac{\delta}{\delta A_{\mu}^{a}} \Gamma \neq 0
$$

a violation of Ward identities, which is most often linked to a breakdown of the renormalizability of the theory. Their vanishing is therefore a necessary ingredient to the consistency of the effective theory. The symmetries in question are the gauge symmetries of abelian and nonabelian gauge theories, as well as the diffeomorphism invariance which is present in the general relativistic formulation of gravity [60]. The subject of anomalies is a topic of its own right and we can only sketch the general outline and finally collect a set of practical formulas which are used to determine the irreducible parts of anomalies in four- and sixdimensional field theories.

The occurrence of quantum corrections that violate classical gauge invariances in four dimensions is due to the triangle diagram of the Adler-Bell-Jackiw anomaly, where a chiral fermion runs in the loop, coupling in a parity violating fashion to external gauge bosons or gravitons. Higher perturbative corrections do not occur by the Adler-Bardeen theorem. In $d$ dimensions the corresponding diagrams involve $d / 2+1$ external bosons. The possible existence of such anomalies can roughly be classified by the properties of the representations of Clifford algebras in the various dimensions. In odd dimensions there are no chiral fermions anyway and no anomalies either. In $d=4 k$ the CPT symmetry of the spectrum relates left-handed particles and right-handed anti-particles such that no anomalies exist in the gravitational sector where only the net number of leftand right-handed fermions matters, whereas gauge anomalies may occur whenever the representations under the gauge group differ. As CPT induces a complex conjugation of the representation, only gauge groups with complex representations can be anomalous. For our purposes this means that in four dimensions we only need to check for anomalies of the gauge symmetry of unitary groups while symplectic and orthogonal groups are harmless. In $d=4 k+2$ dimensions CPT maps left-handed particles to left-handed anti-particles, possibly of a different representation under the gauge group, and parity violation may refer to the gravitational and gauge sector. In addition to the fermions, 
there is also another source for anomalies in supergravity theories and string theory, which is usually absent in pure gauge theory models, a self-dual antisymmetric 2-form tensor $B$-field. Via a tree level coupling

$$
B \wedge \operatorname{Tr} F^{d / 2-1} \quad \text { and } \quad \tilde{B} \wedge \operatorname{Tr} F^{2}
$$

to the field strength $F$ and similarly to the curvature tensor $R$, together with the Hodge dual $(d-4)$-form $\tilde{B}$, it produces a contribution that can interfere with the fermion loop diagram with $d / 2+1$ external gauge bosons or gravitons. From the world volume perspective of a D-brane with chiral fermions in the effective gauge theory the mechanism can be understood as a cancellation of fermion contributions with an anomaly inflow from the bulk via such couplings as $(3.26)[61,62,63]$.

As usual, we shall not be able to check the complete vanishing of the entire anomalous contribution but mostly only determine the irreducible part defined as follows. Mathematically, anomalies can be phrased in terms of the curvature of the gauge and tangent bundle on the space-time manifold, given by their curvature tensors $F$ and $R$. The anomalies are then expressed through invariant polynomials $\hat{I}_{1 / 2}(F, R), \hat{I}_{3 / 2}(F, R)$ and $\hat{I}_{A}(F, R)$ of degree $d / 2+1$ for the contributions of spin $1 / 2$ and spin $3 / 2$ fermions and the antisymmetric tensors. The irreducible part of these polynomials is the term proportional to $\operatorname{Tr} F^{d / 2+1}$ or $\operatorname{Tr} R^{d / 2+1}$. If its coefficient vanishes, the remaining reducible part may be treated by a so-called Green-Schwarz mechanism. It requires a factorization of the remaining polynomial which then takes the correct form to be absorbed by a modification of the gauge transformation of the $B$-field. This mechanism is very involved in detail and an explicit check is very demanding, as it not only requires the factorization but also a number of numerical coefficients to come out with a very particular value. Its discovery was actually a very crucial step to introduce the $S O(32)$ string theory in ten dimensions as a viable candidate for a fundamental theory with a consistent low energy approximation [64]. It was first found in [65] that besides the $B$-field which is present in ten dimensions also other tensor fields may participate in the anomaly cancellation mechanism in lower dimensions, which is also the case in the models we are studying. We shall take reference to this mechanism when we show that the couplings of the fourdimensional axions in toroidal type I models with background gauge fields come out with the correct normalization in section 3.3.4. This is required by the cancellation of leftover abelian and mixed abelian-nonabelian gauge anomalies in four dimensions. A very important consequence of the removal of such anomalies by a Green-Schwarz mechanism is the generation of a mass term for the gauge boson. The respective $U(1)$ decouples from the effective theory below the string scale while the gauge boson "eats" the axion in getting a mass. Both thus disappear from the tree level massless spectrum [66].

Let us now collect a number of useful formulas, see for instance [67] for more details. Concerning the sixdimensional gravitational anomaly we first notice that all models we shall be considering have a supersymmetric closed string sector which only via interactions with open strings may eventually notice the breaking of supersymmetry on the D-branes. Thus, we prefer to count the contributions to the irreducible gravitational anomaly in terms of sixdimensional $\mathcal{N}=1$ supermultiplets, the hyper-, vector- and 
tensormultiplets $H, V$ and $T$. The formula

$$
n_{H}-n_{V}+29 n_{T}=273
$$

is the simple counting rule that allows to check the vanishing of the irreducible contribution. The 273 on the right-hand-side summarizes the effects of the supergravity multiplet which need to be canceled by the additional matter content on the left-hand-side. The nonabelian gauge anomalies are checked for by converting all contributions into traces over field strengths in the fundamental representation of the gauge group, denoted by $\operatorname{tr} F^{d / 2+1}$. In six dimensions one has

$$
\begin{aligned}
\operatorname{Tr}_{\mathbf{a d j}} F^{4} & =2 N \operatorname{tr} F^{4}+6\left(\operatorname{tr} F^{2}\right)^{2}, \\
\operatorname{Tr}_{\mathbf{S}} F^{4} & =(N+8) \operatorname{tr} F^{4}+3\left(\operatorname{tr} F^{2}\right)^{2}, \\
\operatorname{Tr}_{\mathbf{A}} F^{4} & =(N-8) \operatorname{tr} F^{4}+3\left(\operatorname{tr} F^{2}\right)^{2}
\end{aligned}
$$

for the adjoint, symmetric and antisymmetric representations of $U(N)$. The adjoint representations of $S p(N)$ and $S O(N)$ gauge groups are just to be treated like the symmetric and antisymmetric representations above. The similar set of formulas in four dimensions is

$$
\begin{aligned}
\operatorname{Tr}_{\mathbf{a d j}} F^{3} & =2 N \operatorname{tr} F^{3}, \\
\operatorname{Tr}_{\mathbf{S}} F^{3} & =(N+4) \operatorname{tr} F^{3}, \\
\operatorname{Tr}_{\mathbf{A}} F^{3} & =(N-4) \operatorname{tr} F^{3} .
\end{aligned}
$$

These relations are employed to translate the contributions of all fields into the fundamental representation of $U(N)$ to make them comparative.

It is another difficult task and beyond the scope of the present thesis to understand the way how the one-loop string amplitude in type I theory captures all the different anomalies in a single transition function. The basic idea is that the various tensor fields that propagate between the two boundaries decompose into the invariant polynomials

$\hat{I}_{1 / 2}(F, R), \hat{I}_{3 / 2}(F, R)$ and $\hat{I}_{A}(F, R)$ and such measure the anomalous quantum effects. More concretely, the gauge anomalies are only present in the diagrams involving open strings, while the gravitational anomaly also stems from the Klein bottle diagram. For works on this subject see e.g. [68, 69, 70].

\subsection{Large volume compactification}

The study of phenomenological aspects of type I compactifications has surely merits of its own right. While the load of the attention in explicit model building has been directed towards the heterotic string for a long time, mostly concentrated on its version with $E_{8} \times E_{8}$ gauge group, it is appreciated by now that type I theory can have certain advantages. We have already pointed out that the presence of background fluxes can lead to a breaking of supersymmetry at the scale which is set by the vacuum expectation value of the flux. This in turn is quantized in units of the inverse volume of the compact space which conventionally is equal to the string scale, itself near to the Planck scale. Such a scenario does not benefit from supersymmetry at low energies and looses all its 
phenomenological advantages. When it comes to study concrete models of type I strings with such background flux we shall indeed recognize that supersymmetry breaking is unavoidable in most such models.

An obvious way out of the dilemma appears when the string and compactification scales are no longer tied together at the Planck scale. For the heterotic string this would cause problems. Its effective action reduced from ten to four dimensions on a manifold of volume $V_{6}$ roughly looks like

$$
\begin{aligned}
\mathcal{S}_{\text {het }} & =\int d^{10} x \sqrt{-g} e^{-2 \Phi_{\text {het }}}\left(R_{10}+F_{10}^{2}+\cdots\right) \\
& =\int d^{4} x\left(\frac{V_{6}}{l_{\text {het }}^{8} g_{\text {het }}^{2}} R_{4}+\frac{V_{6}}{l_{\text {het }}^{6} g_{\text {het }}^{2}} F_{4}^{2}+\cdots\right) .
\end{aligned}
$$

In particular the gravitational Einstein-Hilbert and the gauge field terms both stem from the string tree level approximation, and thus both come with the same dependence on the dilaton, whose expectation value determines the heterotic string coupling constant $e^{\Phi_{\text {het }}}=g_{\text {het }}$. The powers of the heterotic length scale $l_{\text {het }}$ are added by dimensional analysis. By comparing coefficients this identifies the effective gauge coupling

$$
g_{\mathrm{YM}}^{2}=g_{\text {het }}^{2} \frac{l_{\text {het }}^{6}}{V_{6}}
$$

and the heterotic mass scale relevant to gravitational interactions

$$
M_{\text {het }}=g_{\mathrm{YM}} M_{\mathrm{pl}}
$$

in terms of the fourdimensional Planck mass $M_{\mathrm{pl}}$. One can observe that the difference of heterotic and Planck mass scales is approximately of a factor 100, i.e. $M_{\text {het }} \sim 10^{18}$, and a weakly coupled heterotic string requires the internal volume not to be very much larger than the heterotic length scale. Together this simple analysis tells us that a weakly coupled heterotic string needs to be compactified on a manifold of the size of about the Planck length in order to produce a weakly coupled gauge theory at low energies.

The story changes dramatically when looking at the effective action of type I string theory. The gravitational interaction still comes from a sphere diagram with $\chi=-2$, but the gauge fields are open strings which need a boundary in the world sheet to end on, they appear first in the disc diagram with $\chi=-1$. Also they do not need to propagate in the entire tendimensional space-time but are restricted to the world volume of the $\mathrm{D} p$-branes. When discussing the tadpole cancellation conditions, it appeared that one needs to have D9-branes in the background. Actually one can perform T-dualities which translate type I to the so-called type $\mathrm{I}^{\prime}$ and change D9-branes into lower dimensional $\mathrm{D} p$-branes. Later on we shall explicitly discuss such duality operations. We then have an effective action for type $\mathrm{I}^{\prime}$

$$
\begin{aligned}
\mathcal{S}_{\mathrm{I}} & =\int d^{10} x \sqrt{-g} e^{2 \Phi_{\mathrm{I}}} R_{10}+\int d^{p} x \sqrt{-g_{p}} e^{\Phi_{\mathrm{I}}} F_{p}^{2}+\cdots \\
& =\int d^{4} x\left(\frac{V_{6}}{l_{\mathrm{I}}^{8} g_{\mathrm{I}}^{2}} R_{4}+\frac{V_{p-3}}{l_{\mathrm{I}}^{p-3} g_{\mathrm{I}}} F_{4}^{2}+\cdots\right) .
\end{aligned}
$$


In front of the gauge kinetic term only the compact world volume $V_{p-3}$ not the entire internal volume appears and a factor of the coupling constant disappeared as compared to (3.30). This leads to relations

$$
M_{\mathrm{I}}^{2+n}=g_{\mathrm{YM}}^{4} \frac{V_{p-3}}{l_{\mathrm{I}}^{p-3} V_{n}} M_{\mathrm{pl}}^{2}, \quad g_{\mathrm{YM}}^{2}=g_{\mathrm{I}} \frac{l_{\mathrm{I}}^{p-3}}{V_{p-3}}
$$

for the effective gauge coupling and the scales. Here $V_{n}$ denotes the internal volume of dimension $n=9-p$ transverse to the brane. In order to have weakly coupled type I and gauge theories we still need to have a compact world volume $V_{p-3}$ of the order of the string scale. But the relation for the string scale involves the transverse volume which remains free. In principle, one can choose a large transverse volume

$$
\frac{V_{p-3}}{l_{\mathrm{I}}^{p-3}} \ll V_{n}
$$

to compensate for a small string scale, getting out a large effective fourdimensional Planck scale. Of course, this setting implies an unnatural large hierarchy of compactfication scales lacking any dynamical explanation or deeper justification. How it will be stabilized once supersymmetry is broken also remains an open question. Anyway, this is the basic idea of so-called large extra dimension or large volume compactifications $[71,72,73,74$, 75]. A simple numerical analysis of the effective Newton constant

$$
\gamma_{\mathrm{New}}^{(n)}=g_{\mathrm{YM}}^{4} l_{\mathrm{I}}^{2+n} \frac{V_{p-3}}{l_{\mathrm{I}}^{p-3}}
$$

shows that one can pull down the string scale all way down to the electroweak scale of about $1 \mathrm{TeV}$ by introducing at least 2 large extra dimensions. The experimental tests of gravity at small distances cannot exclude extra dimensions of a size below about $1 \mathrm{~mm}$. Of course, the gauge fields must somehow be prohibited to enter the extra dimensions, a requirement that looks rather unnatural in field theory but is perfectly well understood in string theory in terms of open strings on D-branes.

In the recent past a lot of work has been spent on the construction and study of models with low string scale, many theoretical tests have been performed and as well a number of experiments proposed, for instance, to detect the KK excitations of gravitons, which might be visible at the new string scale in particle detectors as missing energy. A number of obstructions to such a scenario are also being studied. A very recent review of the topic is [76]. For our purposes it will be sufficient to know that there is a mechanism which possibly allows to lower the string scale of type I models down to the electroweak scale such that the breaking of supersymmetry at this scale may still not destroy all the beneficial advantages of low energy supersymmetry. The crucial requirement is that one can leave transverse extra dimensions large.

\subsection{Toroidal compactification with magnetic flux}

Having collected all the necessary ingredients we now come the main purpose of this chapter. We consider type I strings propagating in a space-time which factorizes into a 
four- or sixdimensional noncompact part and an appropriate torus. On the torus there will be a nontrivial gauge bundle with flux in the Cartan subalgebra of the tendimensional gauge group, which breaks the $S O(32)$ down to factors of unitary, orthogonal and symplectic subgroups. Simultaneously, it breaks supersymmetry on the branes and decouples charged fermions of one particular chirality, while the other chirality is left massless. Together this mechanism of turning on fluxes on an otherwise flat compact space is a very straightforward method to engineer gauge groups of phenomenological interest with a matter content of chiral fermions. This can be adapted to some extent to semi-realistic field theoretical models, versions of the Standard Model, GUT models or, for example, left-right symmetric models of the type first discussed in [77]. There do remain drawbacks such as tachyonic scalars in the open string spectrum which are supposed to signal a condensation mechanism towards stable non-threshold bound states of D-branes, and the breaking of supersymmetry at the string scale. Unfortunately, in the present context the latter may not be reconciled in the setting of a model with low string scale and large transverse dimensions. In the next section 3.5 alternative scenarios will be discussed, models which do preserve supersymmetry as well as models which do allow large volume interpretations of string scale supersymmetry breaking.

All of the general features of toroidal type I compactifications with magnetic flux have already been addressed in chapter 2 . We now only have to patch everything together. The crucial technical step to prove the consistency of the models is the tadpole cancellation condition that has been demonstrated for the tendimensional noncompact type I string theory already. One only needs to implement the effects of the compactification on the torus and those of the flux, i.e. adapt the bosonic zero-mode spectrum accordingly. Given a solution to the cancellation of massless RR tadpoles one can compute the massless spectra of the model and verify the vanishing of anomalies as a check. In a first section we compute the three amplitudes which contribute to the tadpoles in a generic fashion for $d=4$ or $d=6$. The latter may be of little phenomenological interest but it is always a powerful consistency check on the theory to test the absence of gravitational anomalies in six dimensions. Anomaly cancellation conditions only apply to gauge anomalies, the term $\operatorname{tr} F^{3}$, in four dimensions and can be rather weak. The combination of the gravitational and the nonabelian gauge anomalies in six dimensions referring to the irreducible terms $\operatorname{tr} R^{4}$ and $\operatorname{tr} F^{4}$ is a stronger requirement for the spectrum of chiral fermions and tensor fields. Thus we prefer to test our construction in six dimensions before proceeding to four. We also comment on the internal geometry of the D-branes with their respective flux, apply and specialize the ideas of chapter 2. Finally, we will construct a number of relevant examples.

\subsubsection{Construction}

There are different strategies to compute the three diagrams, Klein bottle, annulus and Möbius strip. We have constructed the relevant boundary states in chapter 2.1 and the cross-cap in (3.24). Such we could proceed as in chapter 2.1 and perform a direct calculation of the tree channel of all three amplitudes. In fact, the correct normalization of the states requires to compare the tree channel results after a modular transformation to the loop channel traces. Therefore, we choose to avoid some of the redundancy, com-

pute the loop traces first and transform back to the tree channel. One can then read 
off the correct normalization of the boundary and cross-cap states directly and find the contribution to the tadpoles at the same time.

The torus is again assumed to factorize into a direct product of twodimensional tori $\mathbb{T}_{(i)}^{2}$ as in $(2.76)$, labeled by an index $i=1, . ., D$ with coordinates $x_{1}^{(i)}$ and $x_{2}^{(i)}$ for a compactfication to four or six, $10-2 D$, dimensions. While the fluctuations of the antisymmetric NSNS tensor $B$ are projected out of the spectrum by the world sheet parity, the quantized Peccei-Quinn shift symmetry

$$
b^{(i)} \equiv b^{(i)} \bmod \mathbb{Z} .
$$

of the action (2.2) compactified on a torus allows to have discrete background values for the real part of the Kähler structure. This can also be read off from requiring the symmetry of the bosonic zero-mode spectrum under the $\Omega$, which implies

$$
b^{(i)}=0 \text { or } \frac{1}{2} \text {. }
$$

For the moment we have set $b^{(i)}$ to zero, but in section 3.4 we shall also consider nonvanishing $B$-field. On the tori there will be sets of $\mathrm{D}_{\mu}$-branes, $\mu=1, \ldots, 16$, which carry individual magnetic flux on their world volume quantized according to

$$
\mathcal{F}_{\mu}^{(i)}=\frac{m_{\mu}^{(i)}}{n_{\mu}^{(i)} T_{2}^{(i)}} .
$$

The action of the world sheet parity $\Omega$ flips the sign of all the fluxes, such that a $\mathrm{D}_{\mu}$-brane is always accompanied by an image $\mathrm{D}_{\mu^{\prime}}$-brane, $\mu^{\prime}=1, \ldots, 16$ under $\Omega$ with

$$
\mathcal{F}_{\mu^{\prime}}^{(i)}=-\mathcal{F}_{\mu}^{(i)}
$$

In order to visualize the configurations of D-branes on the tori we employ the T-duality on all coordinates $x_{2}^{(i)}$ discussed in section 2.4.3. As the real parts $U_{1}^{(i)}$ of the complex structures are continuous moduli of the models we can set them to zero as well without affecting the spectra, gauge groups or any other essential properties of the effective theories. Only the massive KK spectrum is affected. Then the T-duality is particularly simple, inverting the radius $R_{2}$ just leaves the shape of the torus invariant, it is a square in both frames. The $\mathrm{D} 9_{\mu}$-branes present for tadpole cancellation are transformed into $\mathrm{D}(9-D)_{\mu}$-branes at a relative angle

$$
\tan \left(\varphi_{\mu}^{(i)}\right)=\mathcal{F}_{\mu}^{(i)}
$$

with respect to the axis $x_{1}^{(i)}$. In particular the values $n_{\mu}^{(i)}=0$ or $m_{\mu}^{(i)}=0$ correspond to pairs of branes along any of the axes. They coincide with their images under $\Omega$. The requirement for the winding numbers to be co-prime means that a brane wrapping any cycle specified by $\left(n_{\mu}^{(i)}, m_{\mu}^{(i)}\right)$ twice has to be treated like two branes winding only a single time. The same T-duality also acts on the world sheet parity via

$$
T_{2} \Omega T_{2}^{-1}=\Omega \mathcal{R}
$$


where $\mathcal{R}$ is a reflection of all the $x_{2}^{(i)}$ directions of the torus. It acts on the $\mathrm{R}$ ground states by a flip of its space-time chirality

$$
\mathcal{R}\left|s_{i}, s_{j}\right\rangle=\left|-s_{i}, s_{j}\right\rangle
$$

$s_{i}= \pm 1 / 2$ labeling the spinor excitations on the torus $i=1, \ldots, D, s_{j}$ those transverse to the action of $\mathcal{R}$. In the literature one sometimes finds the convention to combine $\Omega \mathcal{R}$ with an extra reflection $(-1)^{F_{\mathrm{L}}}$ of the left-moving $\mathrm{R}$ vacuum. We avoid this by using a T-dual GSO projection, defined by the fermionic number operators

$$
\begin{aligned}
& (-1)^{F_{L}}\left|s_{i}, s_{j}\right\rangle=e^{2 \pi i \sum_{i, j}\left(s_{j}-s_{i}\right)}\left|s_{i}, s_{j}\right\rangle, \\
& (-1)^{F_{R}}\left|s_{i}, s_{j}\right\rangle=e^{2 \pi i \sum_{i, j}\left(s_{j}+s_{i}\right)}\left|s_{i}, s_{j}\right\rangle .
\end{aligned}
$$

When dealing with the T-dual picture the symmetry of the brane spectrum under $\Omega$ translates into the requirement to have a setting symmetric with respect to the reflection $\mathcal{R}$, branes with indices $\mu$ and $\mu^{\prime}$ being mapped via

$$
\left(n_{\mu}^{(i)}, m_{\mu}^{(i)}\right) \stackrel{\mathcal{R}}{\longrightarrow}\left(n_{\mu^{\prime}}^{(i)}, m_{\mu^{\prime}}^{(i)}\right)=\left(n_{\mu}^{(i)},-m_{\mu}^{(i)}\right)
$$

onto each other.

Let us now first look at the closed string sector. The fluxes on the D-branes do not enter into the Klein bottle amplitude, such that we get the standard result for type I compactified on a square torus. In particular the amplitude is still vanishing because the closed string sector remains supersymmetric as long as no interactions with open strings are considered. The loop channel Klein bottle amplitude (3.7) can be computed straightforwardly

$$
\mathcal{K}=2^{3-D} c_{D}(1-1) \int_{0}^{\infty} \frac{d t}{t^{(6-D)}} \frac{\vartheta\left[\begin{array}{c}
0 \\
1 / 2
\end{array}\right]^{4}}{\eta^{12}} \prod_{i=1}^{D}\left(\sum_{p, q \in \mathbb{Z}} e^{-\pi t\left(p^{2} / R_{1}^{(i) 2}+q^{2} / R_{2}^{(i) 2}\right)}\right) .
$$

The only difference compared to the noncompact Klein bottle is the discrete KK and winding mass spectrum and the according normalization factor

$$
c_{D}=\frac{V_{10-2 D}}{\left(8 \pi^{2}\right)^{5-D}}
$$

for the integration over continuous momenta. Using the formulas given in appendix D one can easily transform this into the tree channel

$$
\tilde{\mathcal{K}}=2^{9-D} c_{D}(1-1) \int_{0}^{\infty} d l \frac{\vartheta\left[\begin{array}{c}
1 / 2 \\
0
\end{array}\right]^{4}}{\eta^{12}} \prod_{i=1}^{D}\left(R_{1}^{(i)} R_{2}^{(i)} \sum_{p, q \in \mathbb{Z}} e^{-4 \pi l\left(p^{2} R_{1}^{(i) 2}+q^{2} R_{2}^{(i) 2}\right)}\right) .
$$

Here one can read off the normalization of the orientifold state

$$
|\mathrm{O} 9\rangle=2^{(D / 2-4)} c_{D}\left(\prod_{i=1}^{D} \sqrt{R_{1}^{(i)} R_{2}^{(i)}}\right)\left(|\mathrm{O} 9\rangle_{\mathrm{NSNS}}+|\mathrm{O} 9\rangle_{\mathrm{RR}}\right) .
$$


Expanding (3.48) allows to extract the contribution to the massless tadpoles,

$$
\tilde{\mathcal{K}}=2^{9-D} c_{D} \int_{0}^{\infty} d l\left(2^{4} \prod_{i=1}^{D}\left(R_{1}^{(i)} R_{2}^{(i)}\right)+o\left(e^{-\pi l}\right)\right) .
$$

This needs to be canceled by open string contributions.

The first consists in the annulus diagrams of open strings stretching between any two sets of D9-branes which we label by $\mu$ and $\nu$ and which may carry generic flux on their respective world volume. They come with a multiplicity $N_{\mu}$ and $N_{\nu}$ and need to be counted together with their images $\mu^{\prime}$ and $\nu^{\prime}$. The various sectors of open strings are visualized in figure 3.3 using the T-dual picture.

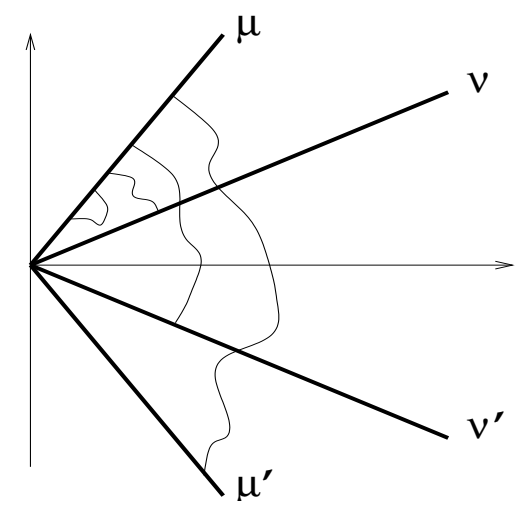

Figure 3.3: Sectors of open strings

The generic case will also include the special situation where some of the flux quantum numbers are vanishing, i.e. D9-branes with vanishing or infinite flux corresponding to horizontal or vertical $\mathrm{D}(9-D)$-branes after T-duality. We start with the contributions of strings with both ends on the same brane. Their KK and winding spectrum in the loop channel has been computed in (2.93) for the present simple choice of tori,

$$
M_{\mu}^{(i) 2}=\frac{\left(p R_{1}^{(i)}\right)^{2}+\left(q R_{2}^{(i)}\right)^{2}}{m_{\mu}^{(i) 2}+\left(n_{\mu}^{(i)} R_{1}^{(i)} R_{2}^{(i)}\right)^{2}} .
$$

Using this the computation is a straightforward generalization of the computation for the flat noncompact space as was performed in greater detail in section B. The result reads

$$
\mathcal{A}_{\mu \mu}=2^{-2} N_{\mu}^{2} c_{D}(1-1) \int_{0}^{\infty} \frac{d t}{t^{(6-D)}} \frac{\vartheta\left[\begin{array}{c}
0 \\
1 / 2
\end{array}\right]^{4}}{\eta^{12}} \prod_{i=1}^{D}\left(\sum_{p, q} e^{-2 \pi t M_{\mu}^{(i) 2}}\right)
$$

and transforms into the tree channel expression

$$
\tilde{\mathcal{A}}_{\mu \mu}=2^{-(D+1)} \mathcal{N}_{\mu}^{2} N_{\mu}^{2}(1-1) \int_{0}^{\infty} d l \frac{\vartheta\left[\begin{array}{c}
1 / 2 \\
0
\end{array}\right]^{4}}{\eta^{12}} \prod_{i=1}^{D}\left(\sum_{p, q} e^{-\pi l \tilde{M}_{\mu}^{(i) 2}}\right)
$$


with $\tilde{M}_{\mu}^{(i)}$ given after applying (D.7) by

$$
\tilde{M}_{\mu}^{(i) 2}=\left(\frac{p^{2}}{R_{1}^{(i) 2}}+\frac{q^{2}}{R_{2}^{(i) 2}}\right)\left(m_{\mu}^{(i) 2}+\left(n_{\mu}^{(i)} R_{1}^{(i)} R_{2}^{(i)}\right)^{2}\right)
$$

and $\mathcal{N}_{\mu}$ the normalization of the corresponding boundary state,

$$
\left|\mathrm{D}_{\mu}\right\rangle=2^{-(D / 2+1)} c_{D}\left(\prod_{i=1}^{D} \frac{m_{\mu}^{(i) 2}+\left(n_{\mu}^{(i)} R_{1}^{(i)} R_{2}^{(i)}\right)^{2}}{\sqrt{R_{1}^{(i)} R_{2}^{(i)}}}\right)^{1 / 2}\left(\left|\mathrm{D}_{\mu}\right\rangle_{\mathrm{NSNS}}+\left|\mathrm{D}_{\mu}\right\rangle_{\mathrm{RR}}\right) .
$$

The latter expression generalizes (2.110) and contains the topological information about the intersection numbers of different branes in the T-dual picture. It implies an extra multiplicity of open string states in the $(\mu, \nu)$ sectors of open strings stretching between different branes.

A very interesting feature of the boundary state (3.55) and the corresponding amplitude (3.52) is the fact that there are massless closed string fields exchanged by the two D-branes which are quantized in terms of the inverse volumes of the tori. In the absence of magnetic flux a $\mathrm{D} p$-brane only couples to a $\mathrm{RR}$ potential $C^{(p+1)}$ of $\operatorname{rank} p+1$, schematically

$$
\mu_{p} \int_{\mathcal{M}_{p+1}} C^{(p+1)}
$$

The presence of 2 -form flux $\mathcal{F}$ on its world volume can induce additional couplings

$$
\mu_{p}^{\prime} \int_{\mathcal{M}_{p+1}} C^{(p-1)} \wedge \mathcal{F}, \quad \mu_{p}^{\prime \prime} \int_{\mathcal{M}_{p+1}} C^{(p-3)} \wedge \mathcal{F} \wedge \mathcal{F}, \quad \text { etc. }
$$

To get the lower dimensional effective theory the coupling of $C^{(10)}$ to a D9-brane which is wrapped on a torus is integrated over the internal space and thus proportional to its volume. This volume factor gets inverted by the T-dualities which map D9-branes to D7-, D5- or D3-branes. Therefore, D9-branes with nonvanishing magnetic flux on their world volume effectively carry the charges not only of normal D9-branes but also those of lower dimensional branes. One can envisage the flux as stemming from a dissolved lower dimensional D-brane inside the D9-brane. In the effective theory this is manifest by the presence of the additional couplings (3.57). An invariant boundary state for a D9-brane with flux in type I will always include two components

$$
\left|\mathrm{D}_{\mu}\right\rangle_{\mathrm{inv}}=\frac{1}{\sqrt{2}}\left(\left|\mathrm{D}_{\mu}\right\rangle+\Omega\left|\mathrm{D}_{\mu}\right\rangle\right)=\frac{1}{\sqrt{2}}\left(\left|\mathrm{D}_{\mu}\right\rangle+\left|\mathrm{D}_{\mu^{\prime}}\right\rangle\right)
$$

Because of (3.45) for nonvanishing $m_{\mu}^{(i)}$ only the bilinear components survive. Hence, in type I a D9-brane with flux can only carry D9- and D5-brane charges, in accord with the fact that there are no D7- or D3-branes in type I. 
The amplitude in the $(\mu, \mu)$ open string sector is still vanishing due to supersymmetry which is only broken by the $(\mu, \nu)$ strings. One can expand the tree channel amplitude to extract the massless divergence

$$
\tilde{\mathcal{A}}_{\mu \mu}=2^{-(D+1)} N_{\mu}^{2} c_{D}(1-1) \int_{0}^{\infty} d l\left(2^{4} \prod_{i=1}^{D}\left(\frac{m_{\mu}^{(i) 2}}{R_{1}^{(i)} R_{2}^{(i)}}+n_{\mu}^{(i) 2} R_{1}^{(i)} R_{2}^{(i)}\right)+o\left(e^{-\pi l}\right)\right) .
$$

In order to compute the $(\mu, \nu)$ contributions to the annulus one can now directly employ the normalized boundary states and get the tree channel expression or do the loop channel trace by regarding the extra factors for the intersection numbers $(2.107)$ for each $\mathbb{T}_{(i)}^{2}$. For simplicity we redefine $I_{\mu \nu}$ by

$$
I_{\mu \nu}=\prod_{i=1}^{D}\left(m_{\mu}^{(i)} n_{\nu}^{(i)}-m_{\nu}^{(i)} n_{\mu}^{(i)}\right)
$$

which is the total intersection number of any two branes $\mu$ and $\nu$. In the loop channel one gets

$$
\begin{aligned}
\mathcal{A}_{\mu \nu}=2^{-2} N_{\mu} N_{\nu} I_{\mu \nu} c_{D} \int_{0}^{\infty} \frac{d t}{t^{(6-D)}} & \sum_{\alpha, \beta \in\{0,1 / 2\}}(-1)^{2(\alpha+\beta)} e^{2 \pi i \beta \sum_{i} \delta_{\mu \nu}^{(i)}} e^{i \pi D / 2} \\
& \times \frac{\vartheta\left[\begin{array}{c}
\alpha \\
\beta
\end{array}\right]^{4-D} \prod_{i=1}^{D} \vartheta\left[\begin{array}{c}
\alpha-\delta_{\mu \nu}^{(i)} \\
\beta
\end{array}\right]}{\eta^{12-3 D} \prod_{i=1}^{D} \vartheta\left[\begin{array}{c}
1 / 2-\delta_{\mu \nu}^{(i)} \\
1 / 2
\end{array}\right]}
\end{aligned}
$$

which transforms into the tree channel amplitude

$$
\begin{aligned}
\tilde{\mathcal{A}}_{\mu \nu}=2^{-1} N_{\mu} N_{\nu} I_{\mu \nu} c_{D} \int_{0}^{\infty} d l(-1)^{D} & \\
& \times \sum_{\alpha, \beta \in\{0,1 / 2\}}(-1)^{2(\alpha+\beta)} \frac{\vartheta\left[\begin{array}{c}
-\beta \\
\alpha
\end{array}\right]^{4-D} \prod_{i=1}^{D} \vartheta\left[\begin{array}{c}
-\beta \\
\alpha-\delta_{\mu \nu}^{(i)}
\end{array}\right]}{\eta^{12-3 D} \prod_{i=1}^{D} \vartheta\left[\begin{array}{c}
-1 / 2 \\
1 / 2-\delta_{\mu \nu}^{(i)}
\end{array}\right]} .
\end{aligned}
$$

We have used

$$
\delta_{\mu \nu}^{(i)}=\frac{\varphi_{\mu}^{(i)}-\varphi_{\nu}^{(i)}}{\pi}
$$

for the shift of oscillator modings and the vacuum energy is given by (A.13). In order to incorporate cases where some $\delta_{\mu \nu}^{(i)}=0$ one needs to employ the relation

$$
\lim _{\psi \rightarrow 0} \frac{2 \sin (\pi \psi)}{\vartheta\left[\begin{array}{c}
1 / 2 \\
1 / 2+\psi
\end{array}\right]}=-\frac{1}{\eta^{3}}
$$

and include a sum over KK momenta and windings on each particular torus. The contribution to the massless RR tadpole is found to be

$$
\tilde{\mathcal{A}}_{\mu \nu}^{(\mathrm{RR})}=2^{-(D+1)} N_{\mu} N_{\nu} c_{D} \int_{0}^{\infty} d l\left(2^{4} \prod_{i=1}^{D}\left(n_{\mu}^{(i)} n_{\nu}^{(i)} R_{1}^{(i)} R_{2}^{(i)}+\frac{m_{\mu}^{(i)} m_{\nu}^{(i)}}{R_{1}^{(i)} R_{2}^{(i)}}\right)+o\left(e^{-\pi l}\right)\right) .
$$


To collect all the contributions from the annulus diagram of an $\Omega$ invariant set of D9branes we have to sum the images

$$
\tilde{\mathcal{A}}_{\mu \nu}^{\mathrm{inv}}=\tilde{\mathcal{A}}_{\mu \nu}+\tilde{\mathcal{A}}_{\mu \nu^{\prime}}+\tilde{\mathcal{A}}_{\mu^{\prime} \nu}+\tilde{\mathcal{A}}_{\mu^{\prime} \nu^{\prime}}
$$

and the entire annulus is then given by

$$
\tilde{\mathcal{A}}^{\text {tot }}=\sum_{\mu, \nu} \tilde{\mathcal{A}}_{\mu \nu}^{\mathrm{inv}}
$$

The third contribution comes from the Möbius strip diagram (3.15). The loop channel trace involves the insertion of $\Omega$ inside the trace such that only strings in the $\left(\mu, \mu^{\prime}\right)$ sectors contribute,

$$
\tilde{\mathcal{M}}^{\text {tot }}=\sum_{\mu} \mathcal{M}_{\mu}
$$

One also has to regard that not all the intersection points of the two branes are invariant under this insertion. In the T-dual picture one can easily convince oneself that only

$$
I_{\mu \mu^{\prime}}^{(\Omega)}=\prod_{i=1}^{D}\left(2 m_{\mu}^{(i)}\right)
$$

of the $I_{\mu \mu^{\prime}}$ intersections are invariant under $\mathcal{R}$. This number can again be computed from purely CFT methods, as was $I_{\mu \nu}$ in section 2.4.4. Given this the loop channel Möbius strip contribution is

$$
\begin{aligned}
\mathcal{M}_{\mu}= \pm 2^{-2} I_{\mu \mu^{\prime}}^{(\Omega)} N_{\mu} c_{D} \int_{0}^{\infty} \frac{d t}{t^{(6-D)}} \sum_{\alpha, \beta \in\{0,1 / 2\}}(-1)^{2(\alpha+\beta)} & \\
& \times \frac{\vartheta\left[\begin{array}{c}
\alpha \\
\beta
\end{array}\right]^{4-D} \prod_{i=1}^{D} \vartheta\left[\begin{array}{c}
\alpha \\
\varphi_{\mu}^{(i)} / \pi+\beta
\end{array}\right]}{\eta^{12-3 D} \prod_{i=1}^{D} \vartheta\left[\begin{array}{c}
1 / 2 \\
\varphi_{\mu}^{(i)} / \pi+1 / 2
\end{array}\right]}
\end{aligned}
$$

and it transforms into the tree channel

$$
\begin{aligned}
\tilde{\mathcal{M}}_{\mu}= \pm 2^{5-D}(-1)^{D} I_{\mu \mu^{\prime}}^{(\Omega)} N_{\mu} c_{D} \int_{0}^{\infty} d l \sum_{\alpha, \beta \in\{0,1 / 2\}}(-1)^{2(\alpha+\beta)} & \\
& \times \frac{\vartheta\left[\begin{array}{c}
\alpha \\
\beta
\end{array}\right]^{4-D} \prod_{i=1}^{D} \vartheta\left[\begin{array}{c}
\alpha \\
\varphi_{\mu}^{(i)} / \pi+\beta
\end{array}\right]}{\eta^{12-3 D} \prod_{i=1}^{D} \vartheta\left[\begin{array}{c}
1 / 2 \\
\varphi_{\mu}^{(i)} / \pi+1 / 2
\end{array}\right]}
\end{aligned}
$$

The contribution to the massless $\mathrm{RR}$ tadpole is

$$
\tilde{\mathcal{M}}_{\mu}^{(\mathrm{RR})}= \pm 2^{5-D} N_{\mu} c_{D} \int_{0}^{\infty} d l\left(2^{4} \prod_{i=1}^{D}\left(n_{\mu}^{(i)} R_{1}^{(i)} R_{2}^{(i)}\right)+o\left(e^{-\pi l}\right)\right) .
$$

We proceed to study the solutions to the tadpole cancellation conditions. All three contributions combine into a perfect square. We then have one condition for each kind of 
charge that is carried by the type I D9-branes with magnetic flux. On a fourdimensional torus there is only the D9-brane charge and the charge of a completely transverse D5brane, i.e. two conditions, one proportional to the overall volume of the torus, the second proportional to its inverse. On a sixdimensional torus, split into three twodimensional tori, a D5-brane has codimension four, i.e. there are three independent D5-brane charges to cancel in addition to the D9-brane charge. The conditions read

$$
\begin{array}{ll}
\prod_{i=1}^{2} R_{1}^{(i)} R_{2}^{(i)}: & \sum_{\mu} N_{\mu} n_{\mu}^{(1)} n_{\mu}^{(2)}=16, \\
\prod_{i=1}^{2} \frac{1}{R_{1}^{(i)} R_{2}^{(i)}}: & \sum_{\mu} N_{\mu} m_{\mu}^{(1)} m_{\mu}^{(2)}=0 .
\end{array}
$$

in six dimensions and

$$
\begin{aligned}
\prod_{i=1}^{3} R_{1}^{(i)} R_{2}^{(i)}: & \sum_{\mu} N_{\mu} n_{\mu}^{(1)} n_{\mu}^{(2)} n_{\mu}^{(3)}=16, \\
R_{1}^{(1)} R_{2}^{(1)} \prod_{i=2,3} \frac{1}{R_{1}^{(i)} R_{2}^{(i)}}: & \sum_{\mu} N_{\mu} n_{\mu}^{(1)} m_{\mu}^{(2)} m_{\mu}^{(3)}=0, \\
R_{1}^{(2)} R_{2}^{(2)} \prod_{i=1,3} \frac{1}{R_{1}^{(i)} R_{2}^{(i)}}: & \sum_{\mu} N_{\mu} m_{\mu}^{(1)} n_{\mu}^{(2)} m_{\mu}^{(3)}=0, \\
R_{1}^{(3)} R_{2}^{(3)} \prod_{i=1,2} \frac{1}{R_{1}^{(i)} R_{2}^{(i)}}: & \sum_{\mu} N_{\mu} m_{\mu}^{(1)} m_{\mu}^{(2)} n_{\mu}^{(3)}=0 .
\end{aligned}
$$

in four dimensions. Note that images with labels $\mu^{\prime}$ are not counted. The total number of D9-brane charges is 32 after including these. One can rewrite the conditions in terms of the homology cycles wrapped by the $\mathrm{D}(9-D)$-branes in the T-dual picture, which produces [78]

$$
\sum_{a} N_{a}\left[\Pi_{a}\right]=32\left[\Pi_{9}\right]
$$

where $a$ labels a basis of all $D / 2$-cycles $\left[\Pi_{a}\right]$ on the $D$ dimensional torus, $N_{a}$ is their multiplicity and $\left[\Pi_{9}\right]$ denotes the particular cycle which corresponds to $\left(n_{\mu}^{(i)}, m_{\mu}^{(i)}\right)=(1,0)$ for $i=1, \ldots, D$, i.e. D9-branes with vanishing flux. Note that by choosing some $n^{(i)}>1$ one can directly reduce the rank of the gauge group, which is usually a hard thing to do in exactly solvable string vacua. For earlier models with a rank reduction due to nonabelian Wilson lines see e.g. [79].

\subsubsection{Internal geometry}

Before going to study the spectra, gauge groups and phenomenological implications of the models thus constructed, we collect some issues concerning the geometry of the internal spaces. These refer to the noncommutativity of the compact world volume of the D-branes, the breaking of supersymmetry in the open string sectors and the perspectives of a compactification with low string scale and large transverse extra dimensions. 
In chapter 2.3 we extensively discussed the emergence of noncommutativity on Dbranes which carry constant background 2-form flux on their world volume. It was shown to be equivalent to an asymmetric rotation of open string coordinates and to be removed when passing to the T-dual picture, where we have D-branes of lower dimension without any flux but with rotated boundary conditions. We have also computed the zero-mode spectrum of KK and winding states (2.93) for the simple choice of a square torus and shown that it can be reinterpreted as the spectrum of rescaled KK momenta without any flux but using the open string metric of Seiberg and Witten. The situation for the internal space we are facing in the models just constructed is only slightly more general. We have sets of D9-branes which carry various different fluxes on their world volumes and therefore experience different noncommutative deformations of their coordinate algebras. In this sense the notion of a unique internal geometry corresponding to a unique internal torus is lost. In fact, every stack of branes supports one factor of the gauge group and thus every such factor comes with a different flux and a different algebra of coordinates. The space probed by open strings then factorizes into algebras $\mathcal{A}_{\mu}$ generated by the local string coordinates, symbolically

$$
\mathbb{T}^{D} \sim \bigotimes_{\mu} \mathcal{A}_{\mu}
$$

each defined with an individual deformation parameter $\Theta_{\mu}$ and associated to a particular factor $G_{\mu}$ of the gauge group, such that charges and deformations are associated.

The second issue, the breaking of supersymmetry is an application of the results presented in section 2.2.1. For simplicity we restrict ourselves to the sixdimensional case first and show that there are no supersymmetric brane configurations which satisfy the tadpole cancellation conditions (3.71). We perform the analysis in the T-dual picture, i.e. we have to look for eigenvalues 1 of the rotation operator that performs the relative rotation of D7-branes. On the $\mathbb{T}^{4}$ with

$$
\varphi_{\mu}^{(1)}-\varphi_{\nu}^{(1)}=\varphi^{(1)}, \quad \varphi_{\mu}^{(2)}-\varphi_{\nu}^{(2)}=\varphi^{(2)}
$$

this requirement boils down to

$$
\varphi^{(1)} \pm \varphi^{(2)}=0 \bmod 2 \pi
$$

for any combination of indices $\mu$ and $\nu$, in particular also for the images $\mu^{\prime}$ and $\nu^{\prime}$ under $\Omega$. It is important to note that angles are measured modulo $2 \pi$, whereas naively one might want to measure the relative angle of two lines in a plane modulo $\pi$. In other words we have to equip the branes with an orientation, which we do by associating the vector $\left(n_{\mu}^{(i)} R_{1}^{(i)}, m_{\mu}^{(i)} R_{2}^{(i)}\right)$ to the respective $\mathrm{D} 7_{\mu}$-brane. Then any set of two different branes needs to be at equal or opposite relative angles on the two tori. Changing the convention for this sign would lead to the exchange of a D7-brane and an anti-D7-brane, which immediately breaks supersymmetry.

Now let us first consider brane configurations without vertical D7-branes and concentrate on the second condition in (3.71), which we call the transversality condition. We are free to choose $m_{\mu}^{(1)} \geq 0$. In order to have any chance to satisfy the transversality condition, there must be at least one pair of branes, $\mathrm{D} 7_{1}$ and $\mathrm{D} 7_{2}$, with $m_{1}^{(2)} \geq 0$ and 
$m_{2}^{(2)} \leq 0$. One can easily convince oneself, that whenever $\mathrm{D} 7_{1}$ and $\mathrm{D} 7_{2}$ have relative angles $\varphi^{(1)}= \pm \varphi^{(2)}$ this cannot be the true for either $\mathrm{D} 7_{1}$ and $\mathrm{D} 7_{2^{\prime}}$ or $\mathrm{D} 7_{1^{\prime}}$ and $\mathrm{D} 7_{2}$. We only need to include vertical D7-branes to complete the proof. They have a positive contribution to the transversality condition in (3.71). In order to get a net negative contribution from D7-branes at angles in $(0, \pi / 2)$ relative to the $X_{1}^{(i)}$ axes, we need $\pm m_{\mu}^{(1)} \geq 0$ and $\mp m_{\mu}^{(2)} \geq 0$ for some $\mu$. These branes have $\varphi^{(1)}=-\varphi^{(2)}$ relative to their mirrors but at best may only have $\varphi^{(1)}=\pi-\varphi^{(2)}$ relative to the vertical D7-branes. Thus, if there is a vertical D7-brane, any other D $7_{\mu}$-brane at some generic angle will see the opposite orientation as does its image $\mathrm{D} 7_{\mu^{\prime}}$ and the two sectors separately preserve supersymmetries of opposite chiralities. The flip of chirality in the open string spectrum refers to a change of the sign of the GSO projection in the loop channel R sector, which produces tachyons in the NS sector likewise. This is the standard situation of a nonsupersymmetric brane-anti-brane system. A similar analysis for compactifications to four dimensions is far more complicated due to the different nature of (3.72). We have not been able to produce any supersymmetric solution to the tadpole cancellation conditions and all the examples we shall present in the following will be nonsupersymmetric.

In the absence of supersymmetry one has to face the presence of a tadpole in the NSNS sector of the theory, which can no longer be canceled simultaneously with the RR contribution. We have discussed in section 3.1.1 that this NSNS vacuum diagram can in principle be removed by a generalization of the Fischler-Susskind mechanism. In fact, this may probably involve a highly nontrivial background for the dilaton and metric fields, and no guarantee of the global stability of the internal geometry is available. One thing we have for sure in type I is that the charge conservation of the RR charge of D9-branes forbids a decay of the configuration into the vacuum. In the absence of tools to tackle these problems more concretely, we shall eventually ignore them in the following. The power of RR tadpole cancellation enables us to construct gauge theories on the D-branes which appear perfectly consistent at least as long as one disregards the gravitational effects induced by bulk-boundary diagrams that transport the illness of the NSNS vacuum into the brane.

A completely different question is, if we can make sense phenomenologically of the gauge theory on the noncompact part of the world volume of our D9-branes, while supersymmetry is broken at the string scale. A way to do this was introduced in section 3.2 , involving large extra dimensions and a string scale at about the electroweak scale. The essential ingredient was to have $\mathrm{D}(9-n)$-branes wrapping the internal space such that one could leave an internal transverse space of dimension $n$ large compared to the string length. This does not work out for the models in question. We would have to perform T-dualities in order to introduce $\mathrm{D}(9-n)$-branes at first. If we then want to have an internal circle transverse to all the $\mathrm{D}(9-n)$-branes these have to be parallel on some particular twodimensional torus. But, as we have shown in section 2.2.2 by analyzing the fermion spectra of open string states, chiral fermions of the fourdimensional effective theory only arise in sectors which have nonvanishing relative angles on all three tori. Therefore chirality and large transverse dimensions are not compatible. We shall later comment on the possibility to remove this obstacle to a direct phenomenological application of the present techniques by introducing nontrivial background spaces like 
orbifolds.

\subsubsection{Spectra in six dimensions}

We are compactifying type I strings on a fourdimensional torus and cancel the tadpoles by introducing stacks of D9-branes with magnetic fluxes according to (3.71). Each stack of such branes supports one factor of the gauge group. Whenever the T-dual set of D7branes is invariant under $\Omega \mathcal{R}$, i.e. they are located along one of the axis, there will be a projection of $\Omega$ on the Chan-Paton labels. We have already met the case of D9-branes with vanishing flux, where

$$
\left(\gamma_{\Omega 9}\right)_{i i^{\prime}} \lambda_{i^{\prime} j^{\prime}}^{9}\left(\gamma_{\Omega 9}^{-1}\right)_{j j^{\prime}}=-\lambda_{j i}^{9}
$$

is the action on the massless vectormultiplet, $\lambda$ an $N_{9} \times N_{9}$ matrix. This projection has the solution $\gamma_{\Omega 9}=1$ for a flat bundle which removes the symmetric part of $\lambda$. The gauge group is then broken to the $S O\left(N_{9}\right)$ subgroup of $U\left(N_{9}\right)$. For vertical D7-branes, former D5-branes, there is a subtle change of the sign [80]

$$
\left(\gamma_{\Omega 5}\right)_{i i^{\prime}} \lambda_{i^{\prime} j^{\prime}}^{5}\left(\gamma_{\Omega 5}^{-1}\right)_{j^{\prime} j}=\lambda_{j i}^{5}
$$

which removes the antisymmetric part and leads to a $S p\left(N_{9}\right)$ gauge group. Note that there are no D7-branes which are vertical on one and horizontal on the second torus, which would refer to true D7-branes in the original type I theory. They do not exist and we have explicitly shown by computing the annulus amplitude of invariant D9-branes (3.58) that the respective charge is absent in type I. On the contrary, there is no such $\Omega$ projection on the Chan-Paton labels of the D9-branes which have nonvanishing and finite flux on their world volume, because they are exchanged with their mirror partners,

$$
\lambda_{i j}^{\mu} \longrightarrow\left(\gamma_{\Omega \mu}\right)_{j i^{\prime}} \lambda_{i^{\prime} j^{\prime}}^{\mu^{\prime}}\left(\gamma_{\Omega \mu}^{-1}\right)_{j^{\prime} i} .
$$

Thus, these branes pairwise support $U\left(N_{\mu}\right)$ gauge groups. Together we get the following set of massless fields from $(\mu, \mu)$ strings:

$$
V_{\mu} \psi_{-1 / 2}^{\mu}|0\rangle_{\mathrm{NS}}, \quad \Phi_{i} \psi_{-1 / 2}^{i}|0\rangle_{\mathrm{NS}}, \quad \chi_{a}|a\rangle_{\mathrm{R}}, \quad \chi_{\dot{a}}|\dot{a}\rangle_{\mathrm{R}}
$$

A vector field and four scalars together with two fermions of opposite chirality, one such set for each adjoint representation of one of the factors of the gauge group

$$
G=S O\left(N_{9}\right) \times S p\left(N_{5}\right) \times \prod_{\mu} U\left(N_{\mu}\right) .
$$

We could also add a little more complication by allowing additional anti-branes. This $(\mu, \mu)$ sector is still supersymmetric with all 16 supercharges inherited from ten dimensions. The above field content is that of a single $\mathcal{N}=2, d=6$ vector multiplet or in terms of $\mathcal{N}=1, d=6$ it splits into

$$
V_{\mu}+\chi_{a} \rightarrow V, \quad 4 \Phi+\chi_{\dot{a}} \rightarrow H
$$

a vector- and a hypermultiplet. The more interesting part is that of $(\mu, \nu)$ strings. The spectrum that emerges from such sectors has been discussed already to some extent in 
section 2.2.2. Whenever there is a nonvanishing relative flux on both tori, there will be a single chiral fermion in six dimensions from the $\mathrm{R}$ sector, together with tachyons and massive scalars from the NS sector. The NS zero-point energy is shifted in accord with (A.13) by

$$
\Delta E_{0}^{\mathrm{NS}}=\frac{1}{2} \sum_{i=1}^{D} \frac{\varphi_{\mu}^{(i)}-\varphi_{\nu}^{(i)}}{\pi}=\frac{1}{2} \sum_{i=1}^{D} \delta_{\mu \nu}^{(i)} .
$$

Let $\delta_{\mu \nu}^{(1)}$ be the largest $\delta_{\mu \nu}^{(i)}$, then the lightest physical state

$$
\psi_{-1 / 2+\delta_{\mu \nu}^{(1)}}|0\rangle_{\mathrm{NS}}
$$

has mass

$$
M_{\mathrm{tach}}^{2}=\Delta E_{0}^{\mathrm{NS}}-\delta_{\mu \nu}^{(1)}=-\frac{1}{2}\left(\delta_{\mu \nu}^{(1)}-\delta_{\mu \nu}^{(2)}\right)
$$

which is negative for nonsupersymmetric configurations. We do not know any exact cure for this instability of the open string vacuum but the proposed condensation mechanism of chapter 2.2.3 may be applicable. It would imply a condensation of the tachyon, which can effectively be described by a deformation of the flat cycle that is defined by the embedding of the respective T-dual D7-branes into the fourdimensional torus. The patterns of gauge symmetry breaking which follow are in nice coincidence with the Higgs mechanism of the Standard Model [78, 81]. The precise masses of these fields depend on the closed strong moduli such as the radii of the torus. Note of course, that these tachyons are somewhat different as compared to tachyons in brane-anti-brane systems, where the masses can be shifted to zero and positive values by introducing Wilson lines along the 1-cycles of the torus. The present tachyons are localized at the intersection locus of the two respective branes, which cannot be removed by continuous deformations.

On the contrary, the chiral fermionic massless spectrum from the $\mathrm{R}$ sector is independent of the moduli. One only needs to classify the intersection points with respect to their mapping under $\Omega$, an invariant $\left(\mu, \mu^{\prime}\right)$ intersection providing a field in the antisymmetric representation $\mathbf{A}_{\mu}$ of the $U\left(N_{\mu}\right)$ due to the projection (3.77) on the Chan-Paton labels, while a pair of intersections mapped upon each other provides also the symmetric representation $\mathbf{S}_{\mu}$ due to the absence of a projection. Any $(\mu, \nu)$ intersection simply gives bifundamental fields. Recall the multiplicities to be given by (3.59) and (3.67) to get table 3.1, which also include the intersections with images $\mu^{\prime}$.

The multiplicity of states is thus given by topological invariants of the D-brane configuration. Whenever the multiplicity is negative formally, one has to pick the $(2,1)$ spinor of opposite chirality taking into account the opposite orientation of the branes at the intersection. As was pointed out earlier, a change of the orientation switches the $\mathrm{RR}$ charge in the tree channel equivalent to the opposite GSO projection in the loop channel. If any two branes are aligned on one of the two tori, the multiplicity becomes zero, which indicates the appearance of a second fermion of opposite chirality from the trivial dimensional reduction along these directions, which is in accord with the index formula (2.41) for the Dirac operator. This part of the spectrum will be nonchiral again, 


\begin{tabular}{|c|c|c|}
\hline Spin & Representation & Multiplicity \\
\hline \hline$(1,2)$ & $\mathbf{A}_{\mu} \oplus \overline{\mathbf{A}}_{\mu}$ & $I_{\mu \mu^{\prime}}^{(\Omega)}$ \\
$(1,2)$ & $\mathbf{A}_{\mu} \oplus \overline{\mathbf{A}}_{\mu} \oplus \mathbf{S}_{\mu} \oplus \overline{\mathbf{S}}_{\mu}$ & $I_{\mu \mu^{\prime}}-I_{\mu \mu^{\prime}}^{(\Omega)}$ \\
$(1,2)$ & $\left(\mathbf{N}_{\mu}, \mathbf{N}_{\nu}\right) \oplus\left(\overline{\mathbf{N}}_{\mu}, \overline{\mathbf{N}}_{\nu}\right)$ & $I_{\mu^{\prime} \nu}$ \\
$(1,2)$ & $\left(\mathbf{N}_{\mu}, \overline{\mathbf{N}}_{\nu}\right) \oplus\left(\overline{\mathbf{N}}_{\mu}, \mathbf{N}_{\nu}\right)$ & $I_{\mu \nu}$ \\
\hline
\end{tabular}

Table 3.1: Chiral massless fermions in $d=6$

while still breaking supersymmetry. We refrain from writing out this part explicitly.

The open string spectrum is complete, and we only need to add the closed string states. This sector completely unaffected by the magnetic flux on the D9-branes and therefore the spectrum is just the same as for an ordinary KK reduction on a torus. It is

$$
G_{\mu \nu}, \quad G_{(i \mu)}, \quad G_{i j}, \quad \Phi,
$$

the graviton, four neutral vectors, and eleven scalars from the NSNS sector together with

$$
C_{\mu \nu}, \quad C_{[i \mu]}, \quad C_{[i j]},
$$

a 2-form, another four neutral vectors and finally six scalars from the RR sector. It is now a straight forward check to see that this spectrum altogether satisfies the cancellation of the irreducible parts of the gravitational and nonabelian anomalies. The first means to check that (3.27) holds and for the second one has to apply (3.28) to add up all contributions, together a very satisfactory check on the consistency of the entire construction. We will not explicitly address the remaining anomalies which need to be canceled by some generalized Green-Schwarz mechanism for the present sixdimensional case but comment on the very similar fourdimensional situation later on. Due to the limited phenomenological use of sixdimensional models we shall not present any explicit examples here but better proceed to apply the procedure to fourdimensional compactifications.

\subsubsection{Fourdimensional models}

The entire construction in four dimensions is in principle very similar to that in six. We have to obey the more complicated conditions (3.72) for the cancellation of RR tadpoles. The stacks of D9-branes with appropriately tuned magnetic fluxes are now characterized by three sets of magnetic and electric quantum numbers, one set per $\mathbb{T}^{2}$. Again the gauge group contains a $U\left(N_{\mu}\right)$ factor for each stack of D9-branes with nonvanishing flux, an $S O\left(N_{9}\right)$ gauge factor for a stack with vanishing flux and an $S p\left(N_{5}\right)$ factor for a stack of D5-branes. The latter can now fill out three different $\mathbb{T}_{(i)}^{2}$ on the sixdimensional torus, such that there may be three independent symplectic factors in the gauge group. This can again be doubled by adding further anti-branes. All the models we shall be able to present will break supersymmetry in the open string sectors of the type $(\mu, \nu)$. Therefore the NSNS tadpole will remain uncanceled and the open string spectrum will contain tachyonic scalars which we like to interpret as Higgs bosons of the effective theory. All 
the remarks made in section 3.3.2 for the fourdimensional internal torus apply here as well. After all, only little remains to be done.

The closed string spectrum is obtained by a further dimensional reduction of the sixdimensional one in (3.86) and (3.87), which we do not need explicitly. The nonchiral and supersymmetric open string $(\mu, \mu)$ sector produces an $\mathcal{N}=4, d=4$ vectormultiplet by dimensional reduction of (3.80) to four dimensions. It carries the adjoint representation of any of the factors of the gauge group as before. At any intersection point of two D6-branes with nonvanishing relative angles on all three tori we get a single fermion of some definite chirality. Whereas, whenever two stacks of D6-branes are aligned on any of the three tori, the respective sector preserves more than $\mathcal{N}=1$ supersymmetry, and its fermion spectrum is nonchiral. Concentrating on the chiral part we again only have to classify the intersections of two stacks of $\left(\mu, \mu^{\prime}\right)$ D6-brane with respect to the action of $\Omega \mathcal{R}$, invariant intersections providing fields in the antisymmetric representation, pairs of intersections mapped upon each other giving antisymmetric and symmetric representations. Any $(\mu, \nu)$ sector provides bifundamental matter. The table 3.2 summarizes the spectrum of massless chiral fermions, displaying the left-handed part and omitting the right-handed anti-particles, which stem from the opposite orientation of the open strings. The $\left(\mu, \nu^{\prime}\right)$ sector has been included together with the $(\mu, \nu)$ sector.

\begin{tabular}{|c|c|}
\hline Representation & Multiplicity \\
\hline \hline$\left(\mathbf{A}_{\mu}\right)_{L}$ & $I_{\mu \mu^{\prime}}^{(\Omega)}$ \\
$\left(\mathbf{A}_{\mu}\right)_{L} \oplus\left(\mathbf{S}_{\mu}\right)_{L}$ & $I_{\mu \mu^{\prime}}-I_{\mu \mu^{\prime}}^{(\Omega)}$ \\
$\left(\mathbf{N}_{\mu}, \mathbf{N}_{\nu}\right)_{L}$ & $I_{\mu^{\prime} \nu}$ \\
$\left(\overline{\mathbf{N}}_{\mu}, \mathbf{N}_{\nu}\right)_{L}$ & $I_{\mu \nu}$ \\
\hline
\end{tabular}

Table 3.2: Chiral massless fermions in $d=4$

Formally, the tables 3.1 and 3.2 are nearly identical, which only reflects the very generic concept of the appearance of chiral fermions at brane intersections. The fourdimensional supersymmetry is no longer chiral and a flip of the chirality implies a conjugation. Hence, a negative multiplicity indicates a field in the conjugate representation. Again one can easily check the absence of the irreducible part of the nonabelian anomaly by using (3.29).

In [78] the Green-Schwarz couplings for a very similar type of construction in type II theory were analyzed. They were mainly concerned with the mixed fourdimensional $U(1)_{\mu}-S U\left(N_{\nu}\right)^{2}$ anomalies which require additional axionic couplings in order to cancel the triangle diagrams of the chiral fermions by tree level exchange of axions. One can indeed observe that there are four axions available in type I, such that we have to expect that a maximum of four anomalous $U(1)$ factors may occur in the gauge group. These axions are given by integrating the RR 2-form $C^{(2)}$ or its Hodge dual 6-form $C^{(6)}$ over 
the internal space

$$
a^{i}=\int_{\mathbb{T}_{(i)}^{2}} C^{(2)}, \quad a=\int_{\mathbb{T}^{6}} C^{(6)} .
$$

The fourdimensional axionic couplings to the two nonabelian gauge bosons are then given by

$$
n_{\nu}^{(i)} a^{i} m_{\nu}^{(j)} m_{\nu}^{(k)} \int_{\mathbb{R}^{4}} F_{\nu} \wedge F_{\nu} \quad \text { and } \quad n_{\nu}^{(1)} n_{\nu}^{(2)} n_{\nu}^{(3)} a \int_{\mathbb{R}^{4}} F_{\nu} \wedge F_{\nu}
$$

the prefactors stemming from the integrals of the field strength and tensor forms over the multiply wrapped D9-branes. In contrast to the type II couplings only terms bilinear in magnetic quantum numbers appear, because fluxes $\nu$ need to be combined with fluxes $\nu^{\prime}$. Together with the analogous coupling terms for the 2 -forms to the abelian gauge boson

$$
N_{\mu} m_{\mu}^{(i)} \int_{\mathbb{R}^{4}} F_{\mu} \wedge \int_{\mathbb{T}_{(j)}^{2} \times \mathbb{T}_{(k)}^{2}} C^{(6)} \quad \text { and } \quad N_{\mu} m_{\mu}^{(1)} m_{\mu}^{(2)} m_{\mu}^{(3)} \int_{\mathbb{R}^{4}} F \wedge C^{(2)},
$$

where the factors $N_{\mu}$ results from the normalization of the $U(1)_{\mu}$ charge. One finds a Green-Schwarz amplitude proportional to

$$
N_{\mu}\left(I_{\mu \nu}+I_{\mu^{\prime} \nu}\right)
$$

which is suitable to cancel the anomaly resulting from triangle diagrams of the bilinear fermions in table 3.2.

A major drawback of the fermion spectra in four dimensions is the multiplicity of generations. In attempting to construct concrete models alike to the Standard Model or some generalization of it, we look for fermions in generations of bifundamental representations, the number of such given by the multiplicity of intersections. But these intersections always come paired with images under $\Omega$ such that given any two stacks of branes we get

$$
N_{\text {gen }}=I_{\mu \nu}+I_{\mu^{\prime} \nu} \in 2 \mathbb{Z}
$$

an even number of generations for the gauge group on the stack $\mu$. This is of course a serious obstruction for phenomenological progress and we shall present a remedy in the following section by turning on the closed string 2 -form modulus $B$ in addition to the magnetic fluxes in the open string sector. Before doing so let us nevertheless go through an example in order to demonstrate the simplicity and power of the approach.

\section{A semi-realistic four generation model}

The fourdimensional tadpole cancellation condition (3.72) implies

$$
\sum_{\mu} N_{\mu} n_{\mu}^{(1)} n_{\mu}^{(2)} n_{\mu}^{(3)}=16
$$

which displays the rescaling of the charge of any D9-brane with nonvanishing flux by its electric quantum numbers. As mentioned already, this provides the opportunity to 
reduce the rank of the gauge group right away by choosing some $n_{\mu}^{(i)}>1$. Therefore, we can envision a model where we already start with the gauge group $U(3) \times U(2) \times U(1)^{r}$, the Standard Model gauge group enlarged by some abelian factors, at the string scale. Of course, one might prefer some intermediate unification scenario and we shall indeed come to present such examples as well. For the moment we just want to show how far one can drive the construction. In order to have three quark generations in the $(\mathbf{3}, \mathbf{2})$ representation of $S U(3) \times S U(2)$, one would need $I_{12}=3$ and $I_{12^{\prime}}=0$. However, as was just pointed out, this is not possible, because $I_{\mu \nu}-I_{\mu \nu^{\prime}}$ is always even. The best we can thus achieve is a model with four generations of quarks and leptons but endowed with suitable quantum numbers to fit with the Standard Model matter content except for the extra generation. The model we found best suited is presented in the following. It has a gauge group $U(3) \times U(2) \times U(1)^{2}$ and results from the following configuration of four stacks of D-branes:

$$
n_{\mu}^{(i)}=\left(\begin{array}{cccc}
1 & 1 & 1 & 1 \\
1 & 1 & 1 & 1 \\
1 & 1 & 1 & 10
\end{array}\right)_{i \mu}, \quad m_{\mu}^{(i)}=\left(\begin{array}{cccc}
0 & 2 & 2 & 0 \\
0 & 1 & -2 & 0 \\
1 & 0 & 0 & 1
\end{array}\right)_{i \mu}
$$

The rows label the three $\mathbb{T}_{(i)}^{2}$ and the columns the four species. This configuration has been illustrated in figure 3.4 .
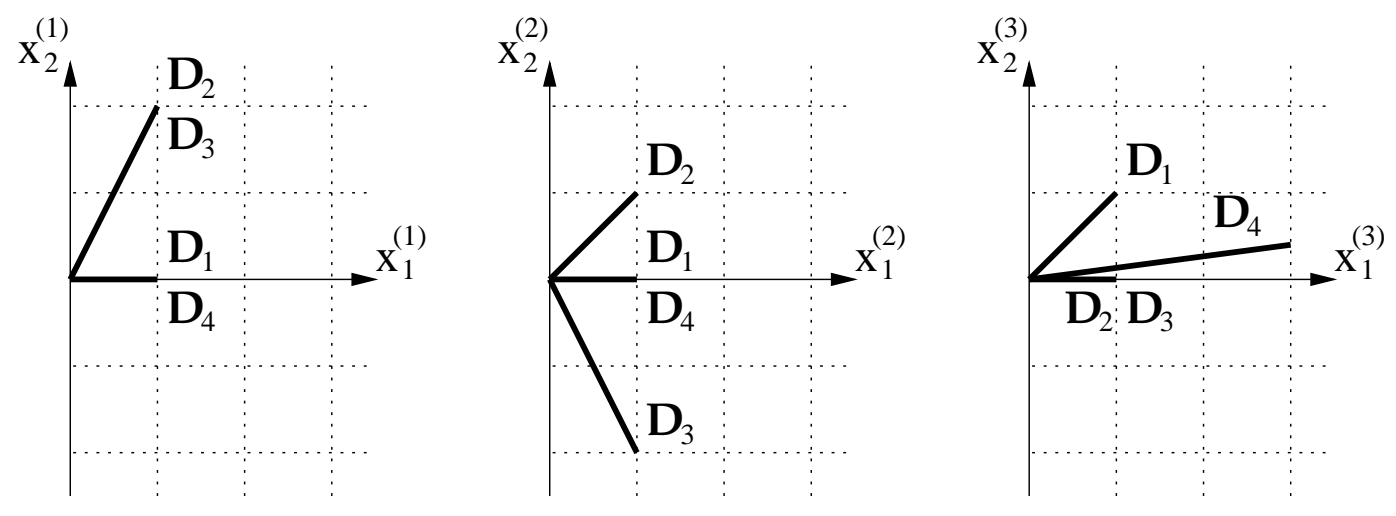

Figure 3.4: Configuration of D6-branes

The resulting chiral massless spectrum is obtained by applying (3.2) straightforwardly. It is shown in table 3.3. Of course, this is not the entire massless spectrum. There are still extra $\mathcal{N}=4$ vectormultiplets present in the supersymmetric $(\mu, \mu)$ sectors which contain pairs of fermions with opposite chirality. Whenever two branes coincide on any of the three tori the respective $(\mu, \nu)$ sector is also nonchiral and the fermions do not fit into the desired Standard Model like spectrum.

By computing the mixed $S U\left(N_{\mu}\right)^{2}-U(1)_{\nu}$ anomalies explicitly one realizes that one of the abelian gauge factors is anomalous, which needs to be cured by the Green-Schwarz mechanism discussed in the previous section. The other three anomaly-free abelian gauge 


\begin{tabular}{|c|c|c|}
\hline $\begin{array}{c}\text { Representation } \\
S U(3) \times S U(2)\end{array}$ & $\begin{array}{c}\text { Charge } \\
U(1)^{4}\end{array}$ & Multiplicity \\
\hline \hline$(\mathbf{3}, \mathbf{2})$ & $(1,1,0,0)$ & 2 \\
$(\mathbf{3}, \mathbf{2})$ & $(1,-1,0,0)$ & 2 \\
$(\overline{\mathbf{3}} \mathbf{1})$ & $(-1,0,-1,0)$ & 4 \\
$(\overline{\mathbf{3}}, \mathbf{1})$ & $(-1,0,1,0)$ & 4 \\
$(\mathbf{1}, \mathbf{2})$ & $(0,1,0,1)$ & 2 \\
$(\mathbf{1}, \mathbf{2})$ & $(0,-1,0,1)$ & 2 \\
$(\mathbf{1}, \mathbf{1})$ & $(0,0,-1,-1)$ & 4 \\
$(\mathbf{1}, \mathbf{1})$ & $(0,0,1,-1)$ & 4 \\
\hline
\end{tabular}

Table 3.3: Four generation spectrum

groups include one,

$$
U(1)_{Y}=\frac{1}{3} U(1)_{1}+U(1)_{3}-U(1)_{4},
$$

which assigns just the correct quantum numbers to all the fermions in order to serve as a hypercharge $U(1)_{Y}$. Diagonalizing appropriately the spectrum finally looks like the one that has been displayed in table 3.4.

\begin{tabular}{|c|c|c|}
\hline $\begin{array}{c}\text { Representation } \\
S U(3) \times S U(2)\end{array}$ & $\begin{array}{c}\text { Charge } \\
U(1)_{Y} \times U(1)^{2}\end{array}$ & Multiplicity \\
\hline \hline$(\mathbf{3}, \mathbf{2})$ & $(1 / 3,1,0)$ & 2 \\
$(\mathbf{3}, \mathbf{2})$ & $(1 / 3,-1,0)$ & 2 \\
$(\overline{\mathbf{3}}, \mathbf{1})$ & $(-4 / 3,0,-1)$ & 4 \\
$(\overline{\mathbf{3}}, \mathbf{1})$ & $(2 / 3,0,1)$ & 4 \\
$(\mathbf{1}, \mathbf{2})$ & $(-1,1,0)$ & 2 \\
$(\mathbf{1}, \mathbf{2})$ & $(-1,-1,0)$ & 2 \\
$(\mathbf{1}, \mathbf{1})$ & $(0,0,-1)$ & 4 \\
$(\mathbf{1}, \mathbf{1})$ & $(2,0,1)$ & 4 \\
\hline
\end{tabular}

Table 3.4: Four generation Standard Model spectrum

This is a semi-realistic, nonsupersymmetric four generation spectrum with properties very close to the Standard Model spectrum. It has two gauged flavour symmetries and features right-handed neutrinos. As already pointed out above, in order to determine the Higgs sector we would have to investigate the bosonic NS sector. However, this is not universal but depends on the radii of the torus via the magnetic flux which is quantized in terms of the inverse volume. We shall not pursue this further as the phenomenological 
applicability is still limited by the impossibility of a large volume scenario with low string scale anyway. In this sense, the phenomenological aspects presented here should not be overestimated and the fundamental problems, tachyon condensation and NSNS tadpoles in the absence of protective supersymmetry, should be kept in mind.

\section{A GUT model}

One may also be interested in searching for scenarios where the Standard Model is not generated already at the string scale, but, as an intermediate step, one first has a GUT unification of gauge groups which only later breaks down to the Standard Model by some undetermined field theoretical mechanism. In fact, in [9] the tuning of internal fluxes on a torus were considered as a mechanism for such model building for the first time. By a field theoretical approach and without the exact string theoretical computations for tadpole cancellation and spectra at hand the results were determined from the cancellation of nonabelian anomalies and the index theorem for the Dirac operator. Then a three generation GUT model with $S U(5)$ gauge group was constructed, which we now like to revisit in order to show how the precise string theoretical calculation detects an unexpected extra multiplicity in the chiral spectrum. It is indispensable to include this extra factor, which can be seen by applying the analogous field theoretical prescription in six dimensions where this deficit would cause gravitational anomalies.

The entire gauge group of the present model is $G=U(5) \times U(3) \times U(4) \times U(4)$. It has still rank 16, as the rank reduction mechanism also escapes the field theoretical analysis. We then have to choose all $n_{\mu}^{(i)}=1$ and one may check that the following choice of $m_{\mu}^{(i)}$ satisfies all tadpole cancellation conditions (3.72),

$$
m_{\mu}^{(i)}=\left(\begin{array}{cccc}
3 & -5 & 1 & -1 \\
1 & 1 & -1 & -1 \\
1 & 1 & 1 & 1
\end{array}\right)_{i \mu} .
$$

The corresponding T-dual configuration of D6-branes is displayed in figure 3.5.
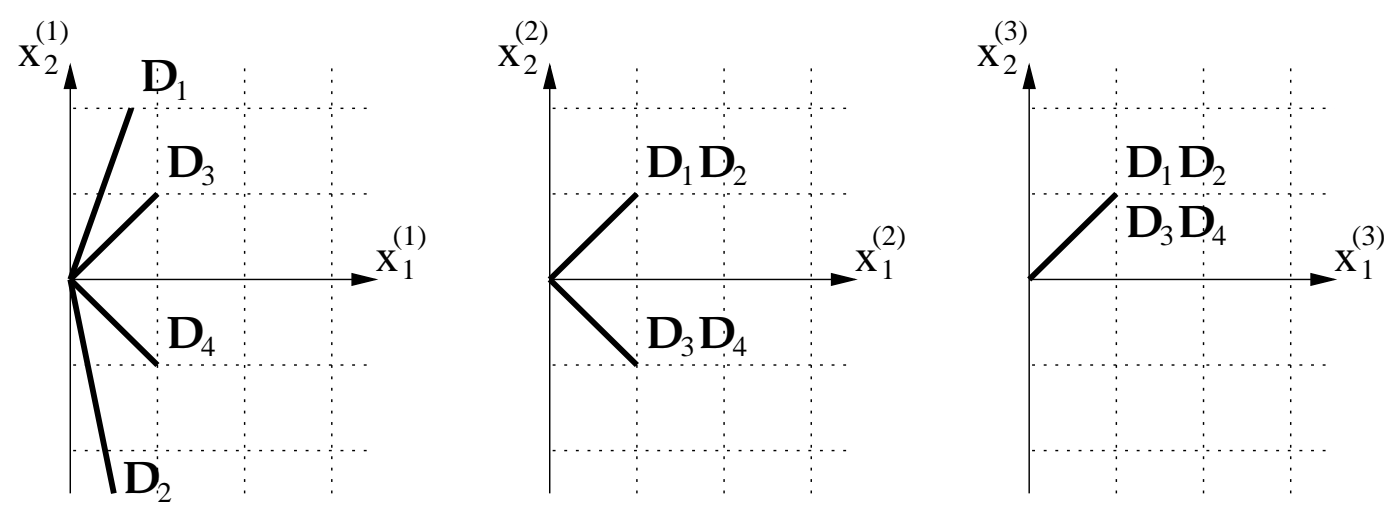

Figure 3.5: ConfigurationD6-branes 
The chiral part of the fermionic massless spectrum is derived by applying table 3.2 and shown in table 3.5 .

\begin{tabular}{|c|c|}
\hline $\begin{array}{c}\text { Representation } \\
U(5) \times U(3) \times U(4) \times U(4)\end{array}$ & Multiplicity \\
\hline \hline$(\mathbf{1 0}, \mathbf{1}, \mathbf{1}, \mathbf{1})$ & 24 \\
$(\mathbf{1}, \mathbf{3}, \mathbf{1}, \mathbf{1})$ & 40 \\
$(\overline{\mathbf{5}}, \overline{\mathbf{3}}, \mathbf{1}, \mathbf{1})$ & 8 \\
$(\mathbf{1}, \mathbf{1}, \overline{\mathbf{6}}, \mathbf{1})$ & 8 \\
$(\mathbf{1}, \mathbf{1}, \mathbf{1}, \mathbf{6})$ & 8 \\
\hline
\end{tabular}

Table 3.5: GUT spectrum

No chiral fermions transform under both the $U(5) \times U(3)$ gauge group and the $U(4) \times$ $U(4)$ gauge group, so that one may figure this second factor as an invisible matter sector, invisible to the GUT group $S U(5)$ in the first factor. There will of course again be nonchiral bifundamental fields which should offset this effect. Picking the $S U(5)$ factor as a GUT gauge group the model has 24 generations of the standard $S U(5)$ GUT model, as a factor of eight stemming from intersection multiplicities of the branes multiplies the matter content of [9]. As all multiplicities come with this factor, it is not detected by only regarding the nonabelian anomaly which also vanishes for three generations of the above field content. But inserting a twodimensional D1-brane probe into the noncompact spacetime should be able to display the presence of a gravitational anomaly in its effective world volume theory [82]. We conclude that in string theory only the spectrum of table 3.5 is viable and the model is actually a 24 generation model.

\subsection{Toroidal compactification with magnetic flux and NSNS $B$-field}

In the previous section we have explored the perspectives of type I string compactifications with magnetic fluxes $\mathcal{F}$ on the various D9-branes. The relatively mild tadpole cancellation conditions offered a very straightforward construction mechanism for a huge variety of models in six or four dimensions. The direct phenomenological applicability suffered from at least two serious defects, the impossibility of having large extra dimensions together with supersymmetry breaking at the string scale and an even number of fermion generations due to the arithmetics of intersection numbers. The first problem requires a nontrivial background geometry, which we turn to in the next section. The second point was related to the symmetry of the brane spectra under the world sheet parity and could easily be resolved within type II string theory [78] in the absence of the $\Omega$ projection. But then the stability of the D9-brane setting is more questionable as no net $\mathrm{RR}$ charge prevents a decay into the vacuum. We shall now introduce a nontrivial background for the closed string NSNS 2-form $B$ in addition to provide a solution of this problem within type I string theory. 


\subsubsection{Construction}

The presence of the NSNS $B$-field in compactifications of type I strings still bears some mysteries. The components of this field that lie in the noncompact part of space-time are projected out of the spectrum by $\Omega$, while the internal part is subject to a Peccei-Quinn shift symmetry such that via (3.38) the constant value

$$
b^{(i)}=0 \text { or } \frac{1}{2}, \quad B_{\mu \nu}^{(i)}=B^{(i)} \epsilon_{\mu \nu}=\frac{b^{(i)}}{T_{2}^{(i)}} \epsilon_{\mu \nu}
$$

for any torus $\mathbb{T}_{(i)}^{2}$ is invariant under a reflection up an integer shift in $b^{(i)}$. One often refers to a background value for $B$ as an NSNS 2-form flux, which is slightly misleading as $B$ is a potential in type II, the potential of the NSNS 3-form field strength $H$. Therefore, its internal components are originally continuous moduli but projected out of the spectrum by $\Omega$, only the two discrete values surviving. Because $B$ is discrete its presence may affect the properties of the model in a drastic and discontinuous way, which it does indeed. The presence of a nonvanishing $B$-field in type I vacua has been studied for instance in $[83,84,85,86,87,88,89]$, and F-theory interpretations have been attempted [90, 91, 92]. The "general knowledge" has emerged that a $B$-field of rank $r$ reduces the rank of the gauge group by a factor of $2^{r / 2}$. Simultaneously, the projection properties such as $(3.77)$ change sign by $(-1)^{r}$, such that the gauge groups are reduced from $S O(32)$ to subgroups accordingly and the representations of matter fields switch from antisymmetric to symmetric and vice versa.

The practical computations have always been performed in a completely different CFT calculus which is very well adapted to keep track of the invariant formulation of the amplitudes and their transformation properties under the modular group but misses geometrical aspects of the D-brane configuration. It starts by constructing the amplitude from abstract arguments and there is no unambiguous and direct way to read off the boundary states which reproduce the transition function afterwards. We shall provide this interpretation in the following and by including magnetic flux $\mathcal{F}$ and NSNS flux $B$ in addition give a more intuitive and geometrical interpretation of the mentioned results. Furthermore we shall find counterexamples to the statement that the rank of the gauge group is reduced by $2^{r / 2}$, which disproves former results to be fully general.

More concretely we shall consider the compactification of type I strings on four- or sixdimensional tori, factorized into twodimensional tori which are characterized by purely

imaginary complex structures as before. As $U_{1}^{(i)}$ remains to be a continuous modulus of the theory, this does not impose any basic restriction on the models. On each $\mathbb{T}_{(i)}^{2}$ we then have $b^{(i)}=0,1 / 2$. Now, we add stacks of $N_{\mu} \mathrm{D} 9_{\mu}$-branes with additional magnetic $F_{\mu}$-flux turned on and look for configurations canceling at least the RR tadpole of the Klein bottle. As for the case with vanishing $B$-field we shall not be able to preserve supersymmetry together with tadpole cancellation.

As explained in section 2.4.3, such a configuration is T-dual to a configuration of $\mathrm{D}(9-D)$-branes intersecting at angles on a torus without background fluxes but with nontrivial complex structure and relative angles between the branes and the $x_{(i)}^{1}$ axes, 
now given by $\varphi_{\mu}^{(i)}=\arctan \left(F_{\mu}^{(i)}\right)$. Thus, in the T-dual model we have a purely geometric picture of what is going on, no background fluxes whatsoever.

The Dirac quantization (2.84) of the magnetic flux on the D9-branes applies to $F$ such that $\mathcal{F}=B+F$ is quantized in terms of half-integer numbers only. We therefore change the notation slightly and denote $F$ by $\left(n_{\mu}^{(i)}, m_{\mu}^{(i)}\right), n_{\mu}^{(i)}, m_{\mu}^{(i)} \in \mathbb{Z}$, not to be confused with $\mathcal{F}$. This addition of $1 / 2$ is the origin of having general integer intersection numbers in open string $\left(\mu, \mu^{\prime}\right)$ sectors. The action of $\Omega$ still reflects the 2 -form on the brane, i.e. $\mathcal{F}$ such that it acts as

$$
\left(n_{\mu}^{(i)}, m_{\mu}^{(i)}\right) \stackrel{\Omega}{\longrightarrow}\left(n_{\mu^{\prime}}^{(i)}, m_{\mu^{\prime}}^{(i)}\right)=\left(n_{\mu}^{(i)},-n_{\mu}^{(i)}-m_{\mu}^{(i)}\right) .
$$

These are essentially all the modifications which are necessary to incorporate the $B$-field into the model.

We will not need to present a comprehensive computation of the one-loop tadpole contributions but merely state the differences as compared to the earlier results with $b^{(i)}=0$. As we are going to analyze the $\mathbb{T}^{4} / \mathbb{Z}_{2}$ orbifold of $K 3$ in the next section and write down all amplitudes explicitly, including the effects of potential $B$-field backgrounds, it is not necessary to do the same here for the toroidal case. The concrete expressions can be obtained from those for the orbifold by just omitting the contributions from the insertion of the orbifold generator in the loop channel.

The presence of $b^{(i)}=1 / 2$ actually enters the amplitudes at three instances. First, the Kaluza-Klein and winding mass spectrum which was formerly given by (2.93) needs to be adapted in accord with (2.92),

$$
M_{\mu}^{(i) 2}=\frac{\left(p R_{1}^{(i)}\right)^{2}+\left(q R_{2}^{(i)}\right)^{2}}{\left(m_{\mu}^{(i)}+b^{(i)} n_{\mu}^{(i)}\right)^{2}+\left(n_{\mu}^{(i)} R_{1}^{(i)} R_{2}^{(i)}\right)^{2}},
$$

second the number of intersection points of two branes changes and finally the number of such intersections which are invariant under $\Omega$. In all three cases the modification can be summarized by noting that the quantum numbers $\left(n_{\mu}^{(i)}, m_{\mu}^{(i)}\right)$ for the $b^{(i)}=0$ case are replaced by $\left(n_{\mu}^{(i)}, m_{\mu}^{(i)}+b^{(i)} n_{\mu}^{(i)}\right)$. The formal origin of this is to be found in the different normalization of the boundary state due to the zero-mode spectrum (3.99). Even though the $B$-field also enters into the closed string sectors the Klein bottle amplitude remains unchanged. By inspecting (2.86) one can see that $B$ only modifies winding states which are projected out by the world sheet parity.

Taken all this, we can just write down the tadpole cancellation conditions by adapting (3.71) and (3.72) in the above sense. They read

$$
\begin{aligned}
& \sum_{\mu} N_{\mu} \prod_{i=1}^{2} n_{\mu}^{(i)}=16 \\
& \sum_{\mu} N_{\mu} \prod_{i=1}^{2}\left(m_{\mu}^{(i)}+b^{(i)} n_{\mu}^{(i)}\right)=0
\end{aligned}
$$


in six dimensions and

$$
\begin{aligned}
& \sum_{\mu} N_{\mu} \prod_{i=1}^{3} n_{\mu}^{(i)}=16 \\
& \sum_{\mu} N_{\mu} n_{\mu}^{(1)} \prod_{i=2,3}\left(m_{\mu}^{(i)}+b^{(i)} n_{\mu}^{(i)}\right)=0 \\
& \sum_{\mu} N_{\mu} n_{\mu}^{(2)} \prod_{i=1,3}\left(m_{\mu}^{(i)}+b^{(i)} n_{\mu}^{(i)}\right)=0 \\
& \sum_{\mu} N_{\mu} n_{\mu}^{(3)} \prod_{i=1,2}\left(m_{\mu}^{(i)}+b^{(i)} n_{\mu}^{(i)}\right)=0
\end{aligned}
$$

in four dimensions. Remember that D9-branes without flux correspond to horizontal $\mathrm{D}(9-D)$-branes which necessarily have even $n_{\mu}^{(i)}$, so that a theory with only D9-branes has a gauge group of rank $16 / 2^{r / 2}$. This is also demonstrated in figure 3.6.

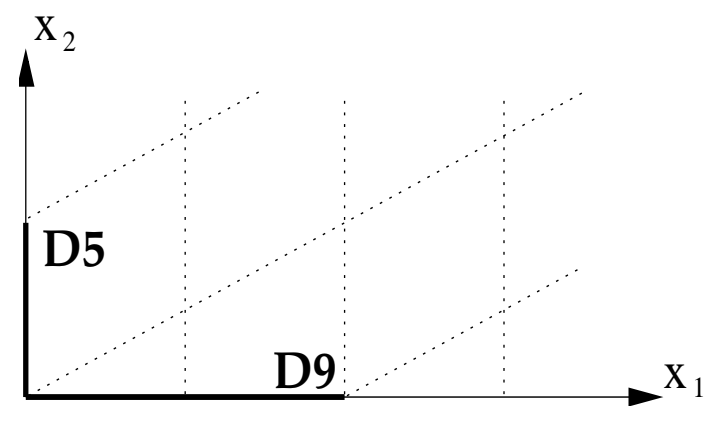

Figure 3.6: T-duals of D5- and D9-branes

Apparently, this rank reduction is not a direct consequence of the background $B$-field, but geometrically follows from the doubled wrapping number of an invariant D9-brane on the torus. In order to have an invariant flux inside its world volume it needs to cover the torus $2^{r / 2}$ times.

The analogies to the case without $B$-field also extend to the spectrum of chiral fermions. It is formally given in tables 3.1 and 3.2 with multiplicities derived from $\left(n_{\mu}^{(i)}, m_{\mu}^{(i)}+b^{(i)} n_{\mu}^{(i)}\right)$ instead of $\left(n_{\mu}^{(i)}, m_{\mu}^{(i)}\right)$. With this modification of the fermion spectrum, we may get any integer

$$
N_{\text {gen }}=I_{\mu \nu}+I_{\mu^{\prime} \nu} \in \mathbb{Z}
$$

for the number of generations.

\subsubsection{A left-right symmetrically unified model}

As an example for a model of a unified theory which includes three generations of matter content, we present a fourdimensional left-right symmetrically unified extension of the Standard Model, a scenario pioneered in [77]. It is realized by four stacks of D6-branes 
with multiplicities $N_{1}=3, N_{2}=N_{3}=2$ and $N_{4}=1$. Only on the second torus we need to turn on a $B$-field $b^{(2)}=1 / 2$. The following choice of quantum numbers

$$
n_{\mu}^{(i)}=\left(\begin{array}{cccc}
1 & 1 & 1 & 1 \\
1 & 1 & 1 & 1 \\
3 & 1 & 1 & 3
\end{array}\right)_{i \mu}, \quad m_{\mu}^{(i)}=\left(\begin{array}{cccc}
0 & 1 & 1 & 0 \\
0 & 1 & -2 & -2 \\
1 & 0 & 0 & 1
\end{array}\right)_{i \mu}
$$

then satisfies the tadpole cancellation conditions (3.101). The brane configuration implies a gauge group $U(3) \times U(2) \times U(2) \times U(1)$. Each one the $S U(2)$ subgroups we assign to the left- respectively right-handed fermions in the spirit of a left-right symmetric version of the Standard Model. The massless spectrum of chiral fermions is found to be

\begin{tabular}{|c|c|c|}
\hline $\begin{array}{c}\text { Representation } \\
S U(3) \times S U(2)_{L} \times S U(2)_{R}\end{array}$ & $\begin{array}{c}\text { Charge } \\
U(1)^{4}\end{array}$ & Multiplicity \\
\hline \hline$(\mathbf{3}, \mathbf{2}, \mathbf{1})$ & $(1,1,0,0)$ & 2 \\
$(\mathbf{3}, \mathbf{2}, \mathbf{1})$ & $(1,-1,0,0)$ & 1 \\
$(\overline{\mathbf{3}}, \mathbf{1}, \mathbf{2})$ & $(-1,0,1,0)$ & 2 \\
$(\overline{\mathbf{3}} \mathbf{1}, \mathbf{2})$ & $(-1,0,-1,0)$ & 1 \\
$(\mathbf{1}, \mathbf{2}, \mathbf{1})$ & $(0,-1,0,1)$ & 3 \\
$(\mathbf{1}, \mathbf{1}, \mathbf{2})$ & $(0,0,-1,-1)$ & 3 \\
\hline
\end{tabular}

Table 3.6: Massless chiral fermions

Computing the mixed $S U\left(N_{\mu}\right)^{2}-U(1)_{\nu}$ anomalies, one realizes that two of the four $U(1)$ factors are anomaly-free. The remaining two required a generalized Green-Schwarz mechanism to cancel the anomaly and decouple the gauge bosons. In particular

$$
U(1)_{B-L}=\frac{1}{3}\left(U(1)_{1}-3 U(1)_{4}\right)
$$

is one of the anomaly-free abelian gauge groups. The model is designed such that the spectrum comes with the correct quantum numbers to use it as the $U(1)_{B-L}$ for the difference of baryon and lepton numbers $B-L$. The diagonalized chiral massless spectrum is shown in table 3.7. This should serve as another example to show how easily semi-realistic spectra can be constructed in this approach. 


\begin{tabular}{|c|c|c|}
\hline $\begin{array}{c}\text { Representation } \\
S U(3) \times S U(2)_{L} \times S U(2)_{R}\end{array}$ & $\begin{array}{c}\text { Charge } \\
U(1)_{B-L} \times U(1)\end{array}$ & Multiplicity \\
\hline \hline$(\mathbf{3}, \mathbf{2}, \mathbf{1})$ & $(1 / 3,1)$ & 2 \\
$(\mathbf{3}, \mathbf{2}, \mathbf{1})$ & $(1 / 3,-1)$ & 1 \\
$(\overline{\mathbf{3}}, \mathbf{1}, \mathbf{2})$ & $(-1 / 3,1)$ & 2 \\
$(\overline{\mathbf{3}}, \mathbf{1}, \mathbf{2})$ & $(-1 / 3,-1)$ & 1 \\
$(\mathbf{1}, \mathbf{2}, \mathbf{1})$ & $(-1,-1)$ & 3 \\
$(\mathbf{1}, \mathbf{1}, \mathbf{2})$ & $(1,1)$ & 3 \\
\hline
\end{tabular}

Table 3.7: Left-right symmetrically unified spectrum

\subsection{Orbifold compactification on K3 with fluxes}

In this final section on geometrical type I compactifications with background fluxes we combine a nontrivial geometrical background together with nontrivial gauge bundles. The compactification manifold is chosen to be one of the supersymmetric orbifolds introduced in section 2.5 to which we also refer for the basic concepts of a string compactification on such spaces. We shall use the most simple example of an orbifold limit of K3, the $\mathbb{T}^{4} / \mathbb{Z}_{2}, \mathbb{Z}_{2}=\{1, \Theta\}$, orientifold first considered in [93, 94] and reinterpreted in terms of D-branes and RR charge cancellation in [80]. General orbifold limits of K3 compactifications of type I have been investigated in [95, 96, 97] while fourdimensional models involving orbifolds of Calabi-Yau 3-folds were pioneered in [98, 99, 91, 100, 101, 102, 103, 104]. The systematics of the latter is less well understood. While in six dimensions all supersymmetric K3 orbifolds of type IIB string theory descend to a consistent vacuum of type I, this does not appear to be the case in four dimensions, where it is well established that the standard projection does not lead to tadpole cancellation in the $\mathbb{Z}_{4}, \mathbb{Z}_{8}, \mathbb{Z}_{8}^{\prime}, \mathbb{Z}_{12}$ cases. The cancellation of the untwisted tadpole in any orbifold of type I is always identical to that on a torus, hence it produces the requirement to include 32 D9-branes. The corresponding $S O(32)$ gauge group may be broken to some subgroup by conditions stemming from twisted sector tadpoles. A further requirement to include 32 D5-branes arises from the presence of orientifold 5-planes for any even $N$.

The standard $\mathbb{Z}_{2}$ orbifold solution we use [80] is chosen to preserve one half of the supersymmetry, 8 supercharges, and produces a chiral spectrum of massless fermions in six dimensions. We shall not be concerned with models using different projections related to discrete torsion $[105,104]$ usually considered to be nongeometric. In order to appreciate the phenomenological progress of the inclusion of magnetic background fields one should notice that the very restrictive consistency conditions of the standard approach usually leave little space for an engineering of realistic low energy physics in type I compactifications on orientifolds. The addition of magnetic flux on the branes and a NSNS $B$-field in the background can be used to break the gauge group and, if desired, supersymmetry in a convenient way while producing chiral fermion spectra of semi-realistic matter content as for the purely toroidal case. The breaking of supersym- 
metry is actually no longer unavoidable as it was in the toroidal case. The presence of nonvanishing curvature just allows to balance the curvature of the gauge bundle with the curvature of the compactification manifold.

\subsubsection{Construction}

Orbifold compactifications of type I strings are conveniently defined by going back to type IIB theory and combine the orbifold group with the world sheet parity. Together they form the orientifold group $\mathbb{Z}_{N} \cup \Omega \mathbb{Z}_{N}$. In the closed string sector one has to project out all states which are not invariant under either the orbifold generator or the world sheet parity and add a twisted sectors for any element of the orbifold group, whereas the open string sector is more subtle. One needs to add the appropriate open strings to cancel the tadpoles which originate from the propagation of twisted fields in addition to the untwisted. The latter are identical to the pure toroidal compactification and therefore cancel by just adding 32 D9-branes into the background. For the former there are two rules of thumb: For any twisted sector contribution one needs to implement a projection on the Chan-Paton degrees of freedom of open strings ending on the D9-branes, i.e. project the gauge group down to some subgroup of $S O(32)$. If $N$ is even additional 32 D5-branes are required to cancel the tadpole that derives from the $\Theta^{N / 2}$ insertion in the Klein bottle loop channel. This sector is T-dual to the D9-brane sector. For the simplest case of a $\mathbb{Z}_{2}$ orientifold we have got 32 D9- and D5-branes and a projection

$$
\lambda_{i j}^{\mu}=\left(\gamma_{\Theta}\right)_{i i^{\prime}} \lambda_{i^{\prime} j^{\prime}}^{\mu}\left(\gamma_{\Theta}^{-1}\right)_{j^{\prime} j}, \quad \operatorname{tr}\left(\gamma_{\Theta}\right)=0
$$

on the Chan-Paton factors. The resulting maximal gauge group is $U(16)_{9} \times U(16)_{5}$. This perturbative CFT analysis is modified by global considerations [106] which we neglect. In the following we shall have to study the modifications of this model by the presence of magnetic background flux on the branes together with a nonvanishing NSNS $B$-field.

The boundary state description of a D-brane on an orbifold space gets modified by first projecting the boundary state on the base space to its invariant component, and second by adding twisted sector components localized at the fixed points of $\Theta$. Transformed to the loop channel these extra terms produce the insertion of the orbifold generator in the loop traces. In the same fashion the cross-cap state of the $\mathbb{Z}_{2}$ orbifold gets a second component, an orientifold O5-plane, which reproduces the contribution of the insertion of $\Theta$ in the loop channel trace. As $\Theta$ reflects all the coordinates of the torus it projects out the vectors of the $\mathcal{N}=2, d=6$ spectrum (3.86) and (3.87) and keeps the graviton, the antisymmetric tensor and the scalars. It has 16 fixed points on the torus which contribute 16 twisted sectors. By doing the T-duality along the $x_{2}^{(i)}$ directions of the torus we can illustrate this in figure 3.7.

Remember that $\mathcal{R}$ reflects at the horizontal axis such that the fixed points need not all be invariant. Also any generic D7-brane which is located at some relative angle to the axis will only pass through some of the fixed points, actually each runs through exactly four. Therefore its boundary state will only carry extra twisted components for the set of fixed points it touches and we shall have to keep track of the 16 twisted sectors individually. As mentioned, the presence of a twisted component in the boundary state signals 

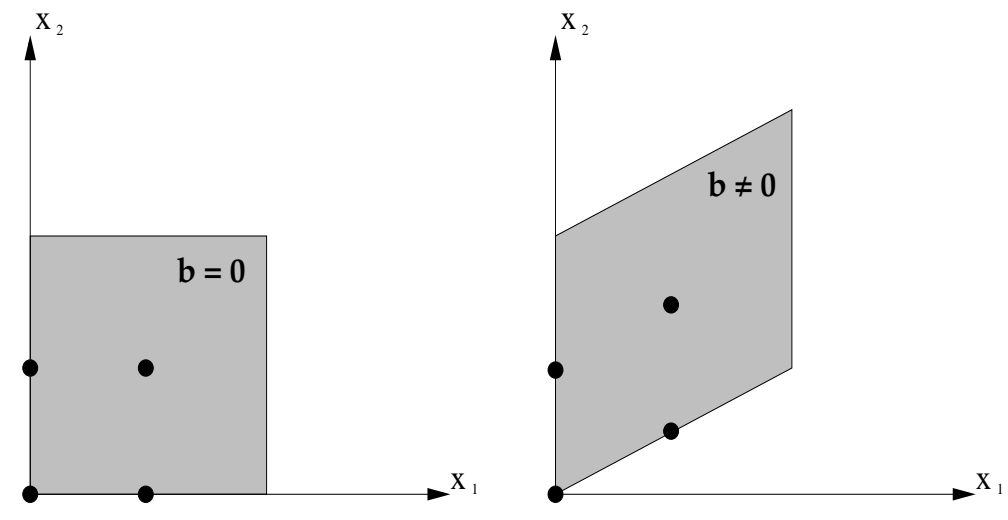

Figure 3.7: T-dual torus with $\mathbb{Z}_{2}$ fixed points

the presence of the $\Theta$ projection in the loop channel, while the presence of a contribution in the Klein bottle or Möbius strip stands for the $\Omega$ projection. Hence, the closed string spectrum of the twisted sector at a fixed point invariant under $\Omega$ has an appropriate $\Omega$ projection, while pairs of fixed points mapped upon each other do not. The open string spectrum arising at the intersection of any two branes has a $\Theta$ projection, if the intersection point coincides with a fixed point of $\Theta$, otherwise no such projection occurs.

In the following we explicitly state the results for the computation of the contributions to the massless RR tadpole. We will not go into the details as much as we did for the toroidal case and restrict ourselves to writing down the tree channel results which are the relevant for the tadpole cancellation conditions. Up to a normalization factor of $1 / 2$ the untwisted contribution is identical to the results for the toroidal case with additional $B$-field turned on. It enters the Klein bottle amplitude by giving rise to different normalization of the O5-plane cross-cap state by a factor of $2^{-r / 2}$. The O5plane corresponds to the $\Omega \Theta$ insertion in the loop which leaves winding states invariant and reflects KK momenta. From (2.86) one can read off the condition

$$
U^{(i)} p_{1}-p_{2}=b^{(i)}\left(q_{1}+U^{(i)} q_{2}\right)
$$

for their vanishing, which implies that $q_{i} \in 2 \mathbb{Z}$, i.e. whenever there is any $B$-flux on a $\mathbb{T}_{(i)}^{2}$ the winding states which contribute to this trace are doubled. This rescaling translates via the modular transformation (D.7) into the normalization factor $2^{-r / 2}$ for the O5-plane. Together, the tree channel for the Klein bottle amplitude reads

$$
\begin{aligned}
& \tilde{\mathcal{K}}= \int_{0}^{\infty} d l\left(\left\langle\mathrm{O} 9\left|e^{-l \mathcal{H}_{\mathrm{cl}}}\right| \mathrm{O} 9\right\rangle+\left\langle\mathrm{O} 5\left|e^{-l \mathcal{H}_{\mathrm{cl}}}\right| \mathrm{O} 5\right\rangle\right) \\
&=2^{6} c_{D}(1-1) \int_{0}^{\infty} d l\left(\frac { \vartheta [ \begin{array} { c } 
{ 1 / 2 } \\
{ 0 }
\end{array} ] ^ { 4 } } { \eta ^ { 1 2 } } \left(\prod_{i=1}^{2}\left(R_{1}^{(i)} R_{2}^{(i)}\right) \prod_{i, j=1}^{2} \sum_{p \in \mathbb{Z}} e^{-4 \pi l p^{2} R_{j}^{(i) 2}}\right.\right. \\
&\left.\left.\quad+\prod_{i=1}^{2}\left(\frac{16^{-b^{(i)}}}{R_{1}^{(i)} R_{2}^{(i)}}\right) \prod_{i, j=1}^{2} \sum_{q \in \mathbb{Z}} e^{-4 \pi l s^{2} /\left(16^{b^{(i)}} R_{j}^{(i) 2}\right)}\right)\right),
\end{aligned}
$$


leading to the following contribution to the massless RR tadpole

$$
\tilde{\mathcal{K}}=2^{6} c_{D}(1-1) \int_{0}^{\infty} d l\left(2^{4}\left(\prod_{i=1}^{2}\left(R_{1}^{(i)} R_{2}^{(j)}\right)+\prod_{i=1}^{2} \frac{16^{-b^{(j)}}}{R_{1}^{(i)} R_{2}^{(i)}}\right)+o\left(e^{-\pi l}\right)\right) .
$$

Note that the mixed overlap of O9- and O5-planes vanishes. The first term is the familiar toroidal term, the second the O5-plane contribution. The charge of the O9-plane state remains unchanged, while the charge of the O5-planes is reduced by the $B$-flux. Together with the insight of the previous section, that invariant D9-branes without any magnetic flux on their world volume wrap the torus twice, this puts a new perspective on the reduction of the rank of the gauge group. The charge of the O5-plane being reduced by the $B$-field it is clear that we need less D5-branes to cancel its RR charge, thus the reduction of the rank of the gauge group supported by D5-branes. Instead, the O9-plane remains unaffected while the invariant D9-branes carry more charge in the presence of the flux by powers of 2 . From this CFT perspective the reduction of the rank of the gauge groups for the D9- and the D5-branes respectively has fairly different origins.

Next we compute the annulus amplitude, which receives contributions from all the open strings stretching among the various D9-branes with individual magnetic fluxes on their world volumes. We have already justified that we need to keep track of all the twisted contributions by treating all fixed points separately and therefore denote the action of the orbifold generator on the Chan-Paton indices of a string ending on a D $9_{\mu^{-}}$ brane by $\gamma_{\Theta \mu}$ letting $\Delta_{\mu}^{(k)}=0,1$ count if the dual D $7_{\mu}$-brane passes through the $k$ th $\mathbb{Z}_{2}$ fixed point in the T-dual picture, $k=1, \ldots, 16$. We also have that

$$
\sum_{k=1}^{16} \Delta_{\mu}^{(k)}=4
$$

independent of $\mu$. The RR sector of the tree channel annulus amplitude of strings with both ends on the same brane is obtained in the loop channel to be

$$
\begin{aligned}
\tilde{\mathcal{A}}_{\mu \mu}^{(\mathrm{RR})=} & \int_{0}^{\infty} d l_{\mathrm{RR}}\left\langle\mathrm{D}_{\mu}^{\mathrm{orb}}\right| e^{-l \mathcal{H}_{\mathrm{cl}}\left|\mathrm{D}_{\mu}^{\mathrm{orb}}\right\rangle_{\mathrm{RR}}} \\
=2^{-4} c_{D} \int_{0}^{\infty} d l\left(N_{\mu}^{2} \frac{\vartheta\left[\begin{array}{c}
1 / 2 \\
0
\end{array}\right]^{4}}{\eta^{12}} \prod_{i=1}^{2}\left(\frac{\tilde{V}_{\mu \mu}^{(i) 2}}{R_{1}^{(i)} R_{2}^{(i)}} \sum_{p, q \in \mathbb{Z}^{2}} e^{-\pi l \tilde{M}_{\mu}^{(i) 2}}\right)\right. & (3.109) \\
& \left.+\frac{\vartheta\left[\begin{array}{c}
1 / 2 \\
0
\end{array}\right]^{2} \vartheta\left[\begin{array}{l}
0 \\
0
\end{array}\right]^{2}}{\eta^{6} \vartheta\left[\begin{array}{c}
0 \\
1 / 2
\end{array}\right]^{2}} \sum_{k=1}^{16} \Delta_{\mu}^{(k)} \operatorname{tr}\left(\gamma_{\Theta \mu}\right)^{2}\right),
\end{aligned}
$$

having used the abbreviation

$$
\tilde{V}_{\mu \nu}^{(i)}=\sqrt{\left(m_{\mu}^{(i)}+b^{(i)} n_{\mu}^{(i)}\right)\left(m_{\nu}^{(i)}+b^{(i)} n_{\nu}^{(i)}\right)+n_{\mu}^{(i)} n_{\nu}^{(i)} R_{1}^{(i) 2} R_{2}^{(i) 2}} .
$$

The zero-mode mass spectrum $\tilde{M}^{(i) 2}$ follows from a modular transformation of (3.99). One can easily read off the massless untwisted and twisted tadpoles

$$
\tilde{\mathcal{A}}_{\mu \mu}^{(\mathrm{RR})}=2^{-4} c_{D} \int_{0}^{\infty} d l\left(2^{4} N_{\mu}^{2} \prod_{i=1}^{2}\left(\frac{\tilde{V}_{\mu \mu}^{(i) 2}}{R_{1}^{(i)} R_{2}^{(i)}}\right)+2^{4} \operatorname{tr}\left(\gamma_{\Theta \mu}\right)^{2}+o\left(e^{-\pi l}\right)\right) .
$$


The annulus amplitude between two different branes in tree channel is

$$
\begin{aligned}
& \tilde{\mathcal{A}}_{\mu \nu}^{(\mathrm{RR})}=\int_{0}^{\infty} d l\left(\mathrm{RR}\left\langle\mathrm{D}_{\mu}^{\mathrm{orb}}\left|e^{-l \mathcal{H}_{\mathrm{cl}}}\right| \mathrm{D}_{\nu}^{\mathrm{orb}}\right\rangle_{\mathrm{RR}}+{ }_{\mathrm{RR}}\left\langle\mathrm{D}_{\nu}^{\mathrm{orb}}\left|e^{-l \mathcal{H}_{\mathrm{cl}}}\right| \mathrm{D}_{\mu}^{\mathrm{orb}}\right\rangle_{\mathrm{RR}}\right) \\
& =2^{-1} c_{D} \int_{0}^{\infty} d l\left(N_{\mu} N_{\nu} I_{\mu \nu} \frac{\vartheta\left[\begin{array}{c}
1 / 2 \\
0
\end{array}\right]^{2} \prod_{i=1}^{2} \vartheta\left[\begin{array}{c}
1 / 2 \\
\delta_{\mu \nu}^{(i)}
\end{array}\right]^{2}}{\eta^{6} \prod_{i=1}^{2} \vartheta\left[\begin{array}{c}
1 / 2 \\
1 / 2+\delta_{\mu \nu}^{(i)}
\end{array}\right]^{2}}\right. \\
& \left.+\frac{\vartheta\left[\begin{array}{c}
1 / 2 \\
0
\end{array}\right]^{2} \prod_{i=1}^{2} \vartheta\left[\begin{array}{c}
0 \\
\delta_{\mu \nu}^{(i)}
\end{array}\right]}{\eta^{6} \prod_{i=1}^{2} \vartheta\left[\begin{array}{c}
0 \\
1 / 2+\delta_{\mu \nu}^{(i)}
\end{array}\right]} \sum_{k=1}^{16} \Delta_{\mu}^{(k)} \Delta_{\nu}^{(k)} \operatorname{tr}\left(\gamma_{\Theta \mu}\right) \operatorname{tr}\left(\gamma_{\Theta \nu}\right)\right)
\end{aligned}
$$

where the number of common fixed points can be written as

$$
\sum_{k=1}^{16} \Delta_{\mu}^{(k)} \Delta_{\nu}^{(k)}=\prod_{k=1}^{2}\left(1+\frac{1}{4}\left(\sum_{\epsilon=0}^{1} e^{\pi i \epsilon\left(n_{\mu}^{(k)}-n_{\nu}^{(k)}\right)}\right)\left(\sum_{\epsilon=0}^{1} e^{\pi i \epsilon\left(m_{\mu}^{(k)}-m_{\nu}^{(k)}\right)}\right)\right) .
$$

The massless contribution to the RR tadpole is

$$
\begin{aligned}
\tilde{\mathcal{A}}_{\mu \nu}^{(\mathrm{RR})}=2^{-1} c_{D} \int_{0}^{\infty} d l & \left(2^{2} N_{\mu} N_{\nu} \prod_{i=1}^{2}\left(\frac{\tilde{V}_{\mu \nu}^{(i) 2}}{R_{1}^{(i)} R_{2}^{(i)}}\right)\right. \\
& \left.+2^{2} \sum_{k=1}^{16} \Delta_{\mu}^{(k)} \Delta_{\nu}^{(k)} \operatorname{tr}\left(\gamma_{\Theta \mu}\right) \operatorname{tr}\left(\gamma_{\Theta \nu}\right)+o\left(e^{-\pi l}\right)\right) .
\end{aligned}
$$

The Möbius amplitude receives only contributions from boundary states invariant under $\Omega$. In tree channel one gets

$$
\begin{aligned}
& \tilde{\mathcal{M}}_{\mu}^{(\mathrm{RR})}=\int_{0}^{\infty} d l\left(\left(\mathrm { RR } \left\langle\mathrm{D}_{\mu}^{\mathrm{orb}}\left|+_{\mathrm{RR}}\left\langle\mathrm{D}_{\mu^{\prime}}^{\mathrm{orb}}\right|\right) e^{-l \mathcal{H}_{\mathrm{cl}}}\left(|\mathrm{O} 9\rangle_{\mathrm{RR}}+|\mathrm{O} 5\rangle_{\mathrm{RR}}\right)\right.\right.\right. \\
& +\left(\mathrm{RR}\left\langle\mathrm{O} 9\left|+{ }_{\mathrm{RR}}\langle\mathrm{O} 5|\right) e^{-l \mathcal{H}_{\mathrm{cl}}}\left(\left|\mathrm{D}_{\mu}^{\text {orb }}\right\rangle_{\mathrm{RR}}+\left|\mathrm{D}_{\mu^{\prime}}^{\text {orb }}\right\rangle_{\mathrm{RR}}\right)\right)\right. \\
& = \pm 2^{5} N_{\mu} I_{\mu \mu^{\prime}}^{(\Omega)} c_{D} \int_{0}^{\infty} d l \frac{\vartheta\left[\begin{array}{c}
1 / 2 \\
0
\end{array}\right]^{2}}{\eta^{6}}\left(\prod_{i=1}^{2}\left(m_{\mu}^{(i)}+b^{(i)} n_{\mu}^{(i)}\right) \frac{\prod_{i=1}^{2} \vartheta\left[\begin{array}{c}
1 / 2 \\
-\varphi_{\mu}^{(i)} / \pi
\end{array}\right]}{\prod_{i=1}^{2} \vartheta\left[\begin{array}{c}
1 / 2 \\
1 / 2-\varphi_{\mu}^{(i)} / \pi
\end{array}\right]}\right. \\
& \left.+\prod_{i=1}^{2} n_{\mu}^{(i)} 4^{-b^{(i)}} \frac{\prod_{i=1}^{2} \vartheta\left[\begin{array}{c}
1 / 2 \\
1 / 2-\varphi_{\mu}^{(i)} / \pi
\end{array}\right]}{\prod_{i=1}^{2} \vartheta\left[\begin{array}{c}
1 / 2 \\
-\varphi_{\mu}^{(i)} / \pi
\end{array}\right]}\right)
\end{aligned}
$$

which leads to the following contribution to the massless RR tadpole

$$
\tilde{\mathcal{M}}_{\mu}^{(\mathrm{RR})}= \pm 2^{5} N_{\mu} c_{D} \int_{0}^{\infty} d l\left(2^{2} \prod_{i=1}^{2}\left(n_{\mu}^{(i)} R_{1}^{(i)} R_{2}^{(i)}\right)+2^{2} \prod_{i=1}^{2} \frac{m_{\mu}^{(i)}+b^{(i)} n_{\mu}^{(i)}}{4^{b^{(i)}} R_{1}^{(i)} R_{2}^{(i)}}+o\left(e^{-\pi l}\right)\right) .
$$

Adding up all the different contributions one gets the general tadpole cancellation conditions

$$
\sum_{\mu} N_{\mu} \prod_{i=1}^{2} n_{\mu}^{(i)}=16
$$




$$
\begin{aligned}
& \sum_{\mu} N_{\mu} \prod_{i=1}^{2}\left(m_{\mu}^{(i)}+b^{(i)} n_{\mu}^{(i)}\right)=16 \prod_{i=1}^{2} 4^{-b^{(i)}}, \\
& \sum_{\mu, \mu^{\prime}} \Delta_{\mu}^{(k)} \operatorname{tr}\left(\gamma_{\Theta \mu}\right)=0, \quad k=1, \ldots, 16 .
\end{aligned}
$$

where in the first two equations the sum is done without counting mirror branes with indices $\mu^{\prime}$ whereas in the third they are counted. The essential differences compared to the toroidal compactification is the presence of a background D5-brane charge, a vacuum charge for a RR 6-form, which is canceled by adding D5-branes or D9-branes with appropriate magnetic flux on their world volume. A topological interpretation of the untwisted tadpole cancellation in terms of T-dual 1-cycles now looks

$$
\sum_{a} N_{a}\left[\Pi_{a}\right]=32\left[\Pi_{9}\right]+2^{5-\operatorname{rk}(B) / 2}\left[\Pi_{5}\right]
$$

$\left[\Pi_{9}\right]$ and $\left[\Pi_{5}\right]$ referring to $(1,0)$ and $(0,1)$ cycles. The third condition describes the consistency condition for the twist in the Chan-Paton gauge bundle. It corresponds to the cancellation of the anomaly inflow at the orbifold singularity with the chiral anomaly in the effective theory on the brane.

Note, that the presence of the $B$-flux does not affect the cancellation of D9-brane charges except for the fact that D9-branes without magnetic flux have even electric quantum numbers. On the other hand, D5-branes without flux may still come in general integer quanta of the elementary charge but the background charge of the O5-plane is reduced. If one does not regard branes with magnetic flux, this conspires to the rule that the resulting number of D9- and D5-branes is divided by the $2^{r / 2}$. But in the presence of nonvanishing magnetic flux it is no longer necessary to reduce the rank strictly in this fashion and we shall take the opportunity to present a supersymmetric model which has $\operatorname{rk}(B)=4$ together with a gauge group of rank $16=32 / 2$.

\subsubsection{Massless spectra}

In this subsection we will derive the generic form of the massless spectrum of the $\mathbb{T}^{4} / \mathbb{Z}_{2}$ orbifold of type I with additional background $B$-field. Let us first summarize the closed string spectrum. According to (A.13) the zero-point energy of both the NSNS and RR twisted sectors are vanishing. In the former we get fermionic zero-modes in the internal directions, while in the latter the fermionic zero-modes of the noncompact space-time survive. Thus in any case the spectrum before the projection consists of $\mathbf{2} \otimes \mathbf{2}=\mathbf{3} \oplus \mathbf{1}$ in terms of representations of the internal or external $S O(4) \cong S U(2) \times S U(2)$. The $\Omega$ projection symmetrizes in the NSNS and antisymmetrizes in the RR sectors [80], such it picks 3 scalars in the NSNS and a single scalar in the RR sector for any fixed point of $\Theta$, which is invariant under $\Omega$, or better under $\Omega \mathcal{R}$ in the dual picture. For any pair of fixed points mapped upon each other by $\Omega \mathcal{R}$ there will be no projection and we get four scalars in the NSNS and a self-dual 2-tensor with a single scalar in the RR sector, which in terms of $\mathcal{N}=1, d=6$ multiplets boils down to the rule:

Invariant fixed point: $H, \quad$ Pair under $\Omega: H+T$. 
Thus, together with the untwisted sector, providing the graviton, 17 scalars and one tensor, combined into a supergravity multiplet, 4 hyper- and 1 tensormultiplet, this is the closed string spectrum:

\begin{tabular}{|c|c|c|}
\hline $\operatorname{rk}(B)$ & Hyper & Tensor \\
\hline \hline 0 & 20 & 1 \\
2 & 16 & 5 \\
4 & 14 & 7 \\
\hline
\end{tabular}

Table 3.8: Closed string spectra

Concerning the open string spectrum we have to distinguish among the different intersection points. The generic solution to the twisted tadpole cancellation is simply

$$
\operatorname{tr}\left(\gamma_{\Theta \mu}\right)=0
$$

for all $\mu$. This implies a breaking of the gauge group

$$
\begin{aligned}
\prod_{\mu} U\left(N_{\mu}\right) & \longrightarrow \prod_{\mu} U\left(N_{\mu} / 2\right) \times U\left(N_{\mu} / 2\right), \\
S O\left(N_{9}\right) & \longrightarrow U\left(N_{9} / 2\right) \\
S p\left(N_{5}\right) & \longrightarrow U\left(N_{5} / 2\right) .
\end{aligned}
$$

For convenience we define $M_{\mu}=N_{\mu} / 2$. The action of $\Omega$ on Chan-Paton indices is explicitly

$$
\gamma_{\Omega \mu}\left(\begin{array}{cc}
A_{1} & A_{2} \\
A_{3} & A_{4}
\end{array}\right)^{T} \gamma_{\Omega \mu}^{-1}=\left(\begin{array}{cc}
A_{4}^{T} & A_{2}^{T} \\
A_{3}^{T} & A_{1}^{T}
\end{array}\right),
$$

the $A_{i}$ being $N_{\mu} \times N_{\mu}$ matrices, $A_{1}$ for $\mu \mu$ strings, $A_{2}$ for $\mu \mu^{\prime}$ strings, etc. It just refers to the switch of the world sheet orientation of the open strings together with the exchange of branes $\mu$ and $\mu^{\prime}$. Hence, the only subsectors which may have $\Omega$ projections are $\left(\mu, \mu^{\prime}\right)$ and $\left(\mu^{\prime}, \mu\right)$ sectors. We then still have $U\left(N_{\mu}\right), S O\left(N_{9}\right)$ and $S p\left(N_{5}\right)$ supported by the respective branes before the orbifold projection. The reflection $\Theta$ leaves all individual branes invariant and thus acts separately on the $N_{\mu} \times N_{\mu}$ factors. Adopting the solution of [80] one has

$$
\gamma_{\Theta \mu}\left(\begin{array}{cc}
B_{1} & B_{2} \\
B_{3} & B_{4}
\end{array}\right) \gamma_{\Theta \mu}^{-1}=\left(\begin{array}{cc}
B_{1} & -B_{2} \\
-B_{3} & B_{4}
\end{array}\right)
$$

This produces the gauge group (3.119). One has to be very careful in applying the appropriate projection and classify all intersection points with respect to their mapping under $\Omega \mathcal{R}, \Theta$ and $\Omega \mathcal{R} \Theta$. Whenever the orientation of the branes at the intersection flips, one further needs to change the chirality of the spinor. Taking all this into account the the matter content of the various open string sectors is summarized in table 3.9. 


\begin{tabular}{|c|c|c|c|}
\hline Sector & Spin & $(\Omega \mathcal{R}(\Theta), \Theta)$ & Matter \\
\hline \hline$\mu \mu$ & $(2,1)$ & $(-, \Theta)$ & $2((\mathbf{a d j}, \mathbf{1}) \oplus(\mathbf{1}, \mathbf{a d j}))$ \\
$\mu \mu$ & $(1,2)$ & $(-, \Theta)$ & $2\left(\mathbf{M}_{\mu}, \mathbf{M}_{\mu}\right) \oplus$ conj. \\
$\mu \mu^{\prime}$ & $(1,2)$ & $(\Omega \mathcal{R}(\Theta), \Theta)$ & $\left(\mathbf{A}_{\mu}, \mathbf{1}\right) \oplus\left(\mathbf{1}, \mathbf{A}_{\mu}\right) \oplus$ conj. \\
$\mu \mu^{\prime}$ & $(2,1)$ & $(\Omega \mathcal{R}(\Theta), \Theta)$ & $\left(\mathbf{M}_{\mu}, \mathbf{M}_{\mu}\right) \oplus$ conj. \\
$\mu \mu^{\prime}$ & $(2,1),(1,2)$ & $(\Omega \mathcal{R}(\Theta),-)$ & $\left(\mathbf{A}_{\mu}, \mathbf{1}\right) \oplus\left(\mathbf{1}, \mathbf{A}_{\mu}\right) \oplus\left(\mathbf{M}_{\mu}, \mathbf{M}_{\mu}\right) \oplus$ conj. \\
$\mu \mu^{\prime}$ & $(2,1),(1,2)$ & $(-,-)$ & $\left(\mathbf{A}_{\mu}, \mathbf{1}\right) \oplus\left(\mathbf{1}, \mathbf{A}_{\mu}\right) \oplus\left(\mathbf{S}_{\mu}, \mathbf{1}\right) \oplus\left(\mathbf{1}, \mathbf{S}_{\mu}\right) \oplus$ \\
& & & $2\left(\mathbf{M}_{\mu}, \mathbf{M}_{\mu}\right) \oplus$ conj. \\
$\mu \nu, \mu \nu^{\prime}$ & $(1,2)$ & $(-, \Theta)$ & $\left(\mathbf{M}_{\mu}, \mathbf{1}, \mathbf{M}_{\nu}, \mathbf{1}\right) \oplus\left(\mathbf{1}, \mathbf{M}_{\mu}, \mathbf{1}, \mathbf{M}_{\nu}\right) \oplus$ conj. \\
$\mu \nu, \mu \nu^{\prime}$ & $(2,1)$ & $(-, \Theta)$ & $\left(\mathbf{M}_{\mu}, \mathbf{1}, \mathbf{1}, \mathbf{M}_{\nu}\right) \oplus\left(\mathbf{1}, \mathbf{M}_{\mu}, \mathbf{M}_{\nu}, \mathbf{1}\right) \oplus$ conj. \\
$\mu \nu, \mu \nu^{\prime}$ & $(1,2),(2,1)$ & $(-,-)$ & $\left(\mathbf{M}_{\mu}, \mathbf{1}, \mathbf{M}_{\nu}, \mathbf{1}\right) \oplus\left(\mathbf{1}, \mathbf{M}_{\mu}, \mathbf{1}, \mathbf{M}_{\nu}\right) \oplus$ \\
& & & $\left(\mathbf{M}_{\mu}, \mathbf{1}, \mathbf{1}, \mathbf{M}_{\nu}\right) \oplus\left(\mathbf{1}, \mathbf{M}_{\mu}, \mathbf{M}_{\nu}, \mathbf{1}\right) \oplus$ conj. \\
\hline
\end{tabular}

Table 3.9: Spectra of $\mathbb{Z}_{2}$ orbifold

We always count single fermions, which, whenever the configurations become supersymmetric, may combine into proper supermultiplets. Of course, they have to be counted with the correct multiplicity for the particular type of intersection. The column $(\Omega \mathcal{R}(\Theta), \Theta)$ denotes whether the intersection point is invariant under $\Omega \mathcal{R}$ or $\Omega \mathcal{R} \Theta$ and $\Theta$, respectively.

When any two branes $\mu$ and $\nu$ pass through the same set of fixed points the conditions for the twisted tadpoles can be expressed in terms of $\operatorname{tr}\left(\gamma_{\Theta \mu}+\gamma_{\Theta \nu}\right)$. In the case of $\nu=\mu^{\prime}$ this results in a larger gauge group $U\left(N_{\mu}\right)$, which happened accidently in all the examples studied in [13], where only the special cases $(n, m) \in(2, \mathbb{Z})$ of non-trivial $F$-fluxes in combination with $B$-fields have been discussed.

\subsubsection{Examples}

In this subsection we will discuss a few examples in some more detail. As a check of the consistency one finds that all of them cancel the sixdimensional irreducible gravitational and nonabelian anomalies by applying (3.27) and (3.28). The T-dual configurations of D7-branes are displayed in figure 3.8, each line corresponding to one of the following three examples. 

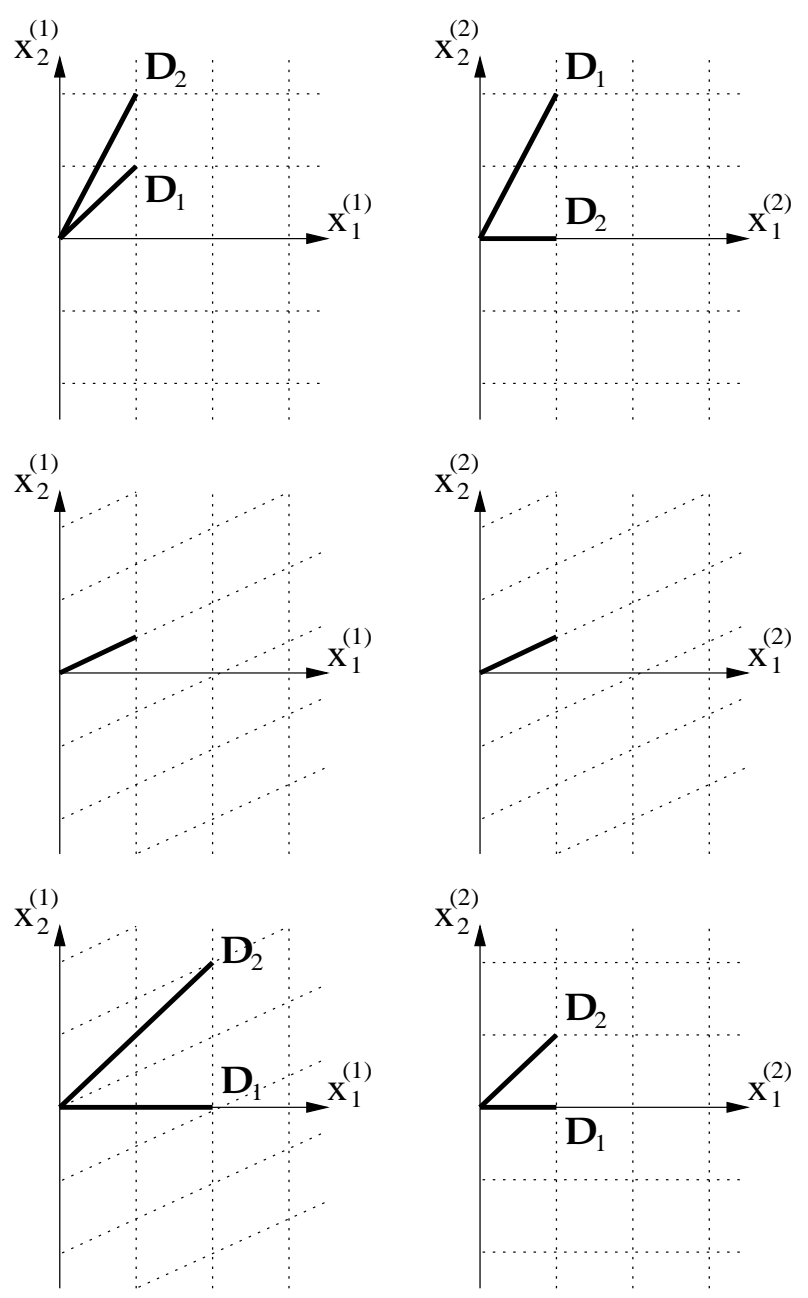

Figure 3.8: Configurations of D7-branes

\section{Generic example}

To present a rather generic case that includes about all complications which may occur we choose two stacks of branes with $N_{1}=N_{2}=8$ and vanishing $B$-field. The following choice of wrapping numbers

$$
n_{\mu}^{(i)}=\left(\begin{array}{ll}
1 & 1 \\
1 & 1
\end{array}\right)_{i \mu}, \quad m_{\mu}^{(i)}=\left(\begin{array}{ll}
1 & 2 \\
2 & 0
\end{array}\right)_{i \mu}
$$

leads to the chiral massless spectrum shown in table 3.10. Together with the 20 hypermultiplets and 1 tensormultiplet from the closed string sector the spectrum in table 4 indeed satisfies the $F^{4}$ and $R^{4}$ anomaly cancellation. In contrast to the toroidal case, the formal vanishing of the intersection number $I_{22^{\prime}}$ does not imply a nonchiral spectrum in this open string sector due to the orbifold projection. 


\begin{tabular}{|c|c|c|}
\hline Sector & Spin & $\begin{array}{c}\text { Representation } \\
U(4) \times U(4) \times U(4) \times U(4)\end{array}$ \\
\hline \hline 11,22 & $(2,1)$ & $2(\mathbf{a d j}, \mathbf{1}, \mathbf{1}, \mathbf{1}) \oplus$ cycl. \\
11 & $(1,2)$ & $2(\mathbf{4}, \mathbf{4}, \mathbf{1}, \mathbf{1}) \oplus$ conj. \\
22 & $(1,2)$ & $2(\mathbf{1}, \mathbf{1}, \mathbf{4}, \mathbf{4}) \oplus$ conj. \\
$11^{\prime}$ & $(1,2)$ & $6(\mathbf{A}, \mathbf{1}, \mathbf{1}, \mathbf{1}) \oplus 6(\mathbf{1}, \mathbf{A}, \mathbf{1}, \mathbf{1}) \oplus 2(\mathbf{4}, \mathbf{4}, \mathbf{1}, \mathbf{1}) \oplus$ conj. \\
$22^{\prime}$ & $(1,2)$ & $3(\mathbf{1}, \mathbf{1}, \mathbf{A}, \mathbf{1}) \oplus 3(\mathbf{1}, \mathbf{1}, \mathbf{1}, \mathbf{A}) \oplus 1(\mathbf{1}, \mathbf{1}, \mathbf{4}, \mathbf{4}) \oplus$ conj. \\
& $(2,1)$ & $3(\mathbf{1}, \mathbf{1}, \mathbf{4}, \mathbf{4}) \oplus 1(\mathbf{1}, \mathbf{1}, \mathbf{A}, \mathbf{1}) \oplus 1(\mathbf{1}, \mathbf{1}, \mathbf{1}, \mathbf{A}) \oplus$ conj. \\
12 & $(2,1)$ & $2(\mathbf{4}, \mathbf{1}, \mathbf{1}, \mathbf{4}) \oplus 2(\mathbf{1}, \mathbf{4}, \mathbf{4}, \mathbf{1}) \oplus$ conj. \\
12 & $(1,2)$ & $4(\mathbf{4}, \mathbf{1}, \mathbf{4}, \mathbf{1}) \oplus 4(\mathbf{1}, \mathbf{4}, \mathbf{1}, \mathbf{4}) \oplus 2(\mathbf{4}, \mathbf{1}, \mathbf{1}, \mathbf{4}) \oplus 2(\mathbf{1}, \mathbf{4}, \mathbf{4}, \mathbf{1}) \oplus$ conj. \\
\hline
\end{tabular}

Table 3.10: Spectrum

\section{$B$-field without rank reduction}

We choose only one stack of D9-branes with $N_{1}=N_{2}=16$ and wrapping numbers $\left(n_{1}, m_{1}\right)=\left(n_{2}, m_{2}\right)=(1,0)$ on the two tori. With a $B$-field of rank four we obtain the simple anomaly free massless spectrum in table 3.11 .

\begin{tabular}{|c|c|c|}
\hline Sector & Spin & $\begin{array}{c}\text { Representation } \\
U(8) \times U(8)\end{array}$ \\
\hline \hline 11 & $(2,1)$ & $2(\mathbf{a d j}, \mathbf{1}) \oplus 2(\mathbf{1}, \mathbf{a d j})$ \\
11 & $(1,2)$ & $2(\mathbf{8}, \mathbf{8}) \oplus$ conj. \\
11 & $(1,2)$ & $(\mathbf{A}, \mathbf{1}) \oplus(\mathbf{1}, \mathbf{A}) \oplus$ conj. \\
\hline
\end{tabular}

Table 3.11: Spectrum

This is an example of a model where the rank of the gauge group is not reduced by $2^{r / 2}=$ 4 but only by a factor of 2 . Together with the 7 tensormultiplets and 14 hypermultiplets from the closed string sector the model satisfies anomaly cancellation. Note, that for an appropriate choice of the radii of the two tori the flux is self-dual and the model becomes supersymmetric.

\section{Type I on $\mathbb{T}^{4} / \mathbb{Z}_{2}$ without D5-branes}

Finally, we discuss a model which was also considered in [13]. We choose two stacks of branes with $N_{1}=N_{2}=4$ and non-zero $B$-field on the first torus $b^{(1)}=1 / 2$ only. A solution to the tadpole cancellation conditions is given by the wrapping numbers

$$
n_{\mu}^{(i)}=\left(\begin{array}{ll}
2 & 1 \\
2 & 1
\end{array}\right)_{i \mu}, \quad m_{\mu}^{(i)}=\left(\begin{array}{cc}
-1 & 0 \\
1 & 1
\end{array}\right)_{i \mu}
$$


where the first brane is a D9-brane without any flux. Therefore, the mirror brane $1^{\prime}$ is of the same kind as the original brane and effectively we have a $U(4)$ gauge group, instead of $U(2)$. Moreover, the second brane and its mirror run through the same set of fixed points. Thus, we can satisfy the twisted tadpole condition by choosing

$$
\operatorname{tr}\left(\gamma_{\Theta 2}\right)=-\operatorname{tr}\left(\gamma_{\Theta 2^{\prime}}\right)
$$

arbitrarily, and there is no orbifold projection on the Chan-Paton labels for the gauge group living on the $\mathrm{D} 9_{2}$-branes. Computing all the intersection numbers and taking the transformation properties of the intersection points into account we obtain the massless spectrum displayed in table 3.12 .

\begin{tabular}{|c|c|c|}
\hline Sector & Spin & $\begin{array}{c}\text { Representation } \\
U(4) \times U(4)\end{array}$ \\
\hline \hline 11,22 & $(2,1)$ & $2(\mathbf{a d j}, \mathbf{1}) \oplus 2(\mathbf{1}, \mathbf{a d j})$ \\
11 & $(1,2)$ & $2(\mathbf{A}, \mathbf{1}) \oplus$ conj. \\
$22^{\prime}$ & $(1,2)$ & $8(\mathbf{1}, \mathbf{A}) \oplus 2(\mathbf{1}, \mathbf{S}) \oplus$ conj. \\
12 & $(1,2)$ & $4(\mathbf{4}, \mathbf{4}) \oplus$ conj. \\
\hline
\end{tabular}

Table 3.12: Spectrum

This completely agrees with the result obtained in [13], but one could also use the generic gauge bundle with

$$
\operatorname{tr}\left(\gamma_{\Theta 2}\right)=\operatorname{tr}\left(\gamma_{\Theta 2^{\prime}}\right)=0
$$

and still obtain a consistent model.

\subsection{Further phenomenological perspectives}

In the works $[78,81]$ the phenomenology of the present class of compactifications has been developed further and a number of attractive properties were elucidated. These include the stability of the proton, the possibility of large hierarchies of Yukawa couplings and the concrete realization of a large volume scenario with low string scale. The presentation in the mentioned works is actually very general and does not refer particularly to type I models. The authors discuss the properties of string compactifications where the gauge theory is realized by sets of D9-branes with magnetic fluxes on their internal world volume which is compactified on a sixdimensional torus or on orbifolds thereof. In their concrete examples they specialize to type II models rather than type I, but the relevant phenomenological features of the gauge sector of the effective low energy field theory appear to be fully generic. Because they always use the T-dual version of the models at hand, the description in terms of intersecting brane worlds of D6-branes wrapped on a torus, we shall also do so in this section not to confuse the notation. It a simple matter of applying the T-duality explained in great detail in section 2.4.3 to get 
back to the "flux" picture.

The gauge couplings $g_{\mathrm{YM} \mu}$ of the effective theory on the $\mathrm{D} 6_{\mu}$-branes is given by a trivial dimensional reduction of the string coupling $g_{\mathrm{I}}$ along the branes,

$$
\frac{1}{g_{\mathrm{YM} \mu}^{2}} \sim \frac{1}{g_{\mathrm{I}}} \frac{V_{\mu}}{l_{\mathrm{I}}^{3}}
$$

where $V_{\mu}$ is the volume of the cycle, which is measured in terms of the string length scale $l_{\mathrm{I}}$. For models which break supersymmetry already at the string scale, which refers to all the purely toroidal models we have been discussing, it is mandatory to lower the string mass scale down to the electroweak range in order to avoid the hierarchy problem, just in the manner of a large volume compactification as explained in section 3.2. We have shown that such a setting cannot be achieved for purely toroidal models just as a chiral spectrum demands that the D6-branes do not leave any transverse direction free to be kept large compared to the string scale. Instead, we expect that it will be possible to achieve a large volume configuration in type I by combining a suitable orbifold compactification to six dimensions with additional fluxes on a $\mathbb{T}^{2}$ in the form of

$$
\frac{\mathbb{T}^{4}}{\mathbb{Z}_{3}} \times \mathbb{T}^{2}
$$

The cancellation of the untwisted tadpole for the supersymmetric sixdimensional $\mathbb{Z}_{3}$ orientifold demands the presence of 32 D9-branes together with a particular twist in the Chan-Paton bundle in order to cancel the twisted tadpole as well. While the dimensional reduction on the $\mathbb{T}^{2}$ would lead to a nonchiral $\mathcal{N}=2$ supersymmetric theory in $d=4$, one may turn on magnetic fluxes on this torus to achieve chirality and supersymmetry breaking. The volume of the $\mathbb{T}^{4}$ remains a modulus of the orbifold theory and may be chosen to be large. Upon performing T-dualities to type $\mathrm{I}^{\prime}$ this would then realize a large volume scenario on an orbifold with additional magnetic fluxes in the background. In fact, the concrete calculations for this particular model have not been performed yet. Therefore, we cannot really judge if the freedom available in tuning the gauge groups and spectra will be favourable enough to construct reasonable semi-realistic models.

There are some interesting remarks on the couplings in intersecting brane world models to be made. The Yukawa couplings of the Standard Model Higgs field are of trilinear type. One requires a large hierarchy of the dimensionless coupling constants $g_{\mathrm{Yu}}^{i j k}$ in order to generate the ratios of fermion masses as observed in nature. In the models under consideration such couplings are mediated by open string world sheets that have ends on three different branes since the Higgs and fermion fields are usually localized at different intersection points which need to be connected by the respective world sheet. The tree level contribution has the shape of a triangle and the coupling strength can classically be estimated by its area $A_{i j k}$ through

$$
g_{\mathrm{Yu}}^{i j k} \sim \exp \left(-\frac{A_{i j k}}{\alpha^{\prime}}\right) .
$$

This expression depends on the intersection form as well as on the radii of the various circles. One can easily convince oneself that the number of free parameters involved easily allows to realize classically a desired hierarchy of couplings by tuning the geometry 
appropriately. Of course, a more thorough treatment will have to involve an estimation of the quantum corrections which may modify the setting considerably.

A usual drawback of large volume scenarios with small string scale is the problem of proton stability. In string theory one expects to have four fermion interaction terms of dimension six which mediate the decay of the proton into a single quark. These are usually suppressed by the large mass scale, i.e. the coupling is of the order of the inverse string mass scale. This suppression is offset in a large volume scenario and a remedy needs to be found. As is pointed out in [81] it indeed exists in the present class of models and consists in the fact that the contributions of string diagrams with three changes of boundary conditions along the entire boundary of the world sheet vanish at any order of perturbation theory. Thus, the proton of intersecting brane world models is perturbatively stable to any order of the string coupling.

Let us finally mention that these kinds of models also give rise to a new possible signature in their spectrum of lightest massive excitation, eventually accessible to accelerator experiments. As is most easily demonstrated by using the slightly heuristec formula (2.38) the lightest massive fermions get masses proportional to the angle formed by the two kinds of branes which they stretch between. It was argued in [81] that these states may be lighter than the generic KK excitations of scalar or vector fields. In particular, the existence of these excitations entirely depends on the presence of the magnetic background and thus may distinguish from different kinds of models.

To conclude this section let us summarize that we have developed a very convenient and powerful tool in constructing semi-realistic type I compactifications with many phenomenologically attractive features in their low energy field theory. One still should not forget that there remain conceptual problems. 


\section{Chapter 4}

\section{Type I superstrings with asymmetric symmetries}

In this chapter we proceed to a rather different and more exotic type of compactification. By now we have studied open strings with background fluxes on branes wrapping tori or toroidal orbifolds. The fluxes on the various D9-branes were also shown to have an equivalent description via asymmetric rotations. These applied to D-branes without flux produced the modified boundary conditions (2.10) for such with flux. The world volume of the respective branes turns noncommutative when performing this rotation. A certain T-duality further maps this setting to branes of lower dimensions which are rotated by ordinary rotations so that they are located at various relative angles and have a completely commutative world volume geometry. Just as one can "gauge" ordinary rotations on a torus in an orbifold compactification one can construct type I vacua invariant under asymmetric rotations $\hat{\Theta}$, or, equivalently, under symmetric rotation in a T-dual version where $\Omega$ is replaced by $\Omega \mathcal{R}[107,108,109,19]$. In a more formal way one can think of an orbifold compactification as an equivariant string theory, symmetric with respect to some group that acts "isometrically" on the fields that define this background. Then there is no substantial distinction between symmetric and asymmetric orbifold constructions as being pioneered by [47].

We will be discussing asymmetric orientifolds in six and four dimensions which are just the T-dual of symmetric orientifolds with a modified world sheet projection $\Omega \mathcal{R}$. We restrict ourselves to such models which preserve $\mathcal{N}=1$ supersymmetry in the respective dimension. As opposed to the case of standard symmetric orientifolds [104] we shall be able to present solutions for consistent models in all fourdimensional examples we inspect, which in particular includes the $\mathbb{Z}_{4}$ orbifold. Again we shall jump from the asymmetric exotic picture to the T-dual symmetric and possibly more intuitive picture whenever it appears useful.

The geometry of the internal space, defined by open strings which probe the world volume of the D-branes wrapping the torus, will be very exotic for an asymmetric orientifold of the type generated by $\hat{\Theta}$, a rotation that treats left- and right-moving fields precisely with opposite phases. This operation identifies D9-branes with a certain value for the magnetic flux on their world volume with others which carry different fluxes, the 
identification given by the addition of the angle variables in the T-dual picture

$$
\varphi^{\prime} \equiv \varphi+\varphi^{\prime}
$$

$\tan \left(\varphi^{\prime}\right)$ the flux on some brane and $\varphi$ the rotation angle of $\Theta$. Eventually this may even identify D9-branes and lower dimensional branes e.g. D5-branes, a phenomenon that was also met in $[110,111]$. In earlier toroidal models we had various different D9-branes present on a torus and therefore lost the notion of a unique definition of the internal geometry. On the asymmetric orbifold open strings do not even distinguish between Dbranes with flux from those without, such that commutative and noncommutative spaces are indistinguishable.

\subsection{A class of supersymmetric asymmetric orientifolds}

We now construct asymmetric orientifolds on a four- or sixdimensional torus which factorizes according to $(2.76)$ into $\mathbb{T}_{(i)}^{2}$ with coordinates complexified as in (2.18). For the fourdimensional case we have to perform three T-dualities on $X_{(i)}^{2}$ to obtain a fully symmetric $\Omega \mathcal{R}$ orientifold. This is then rather a symmetry of the type IIA than of the type IIB superstring, since $\mathcal{R}$ also flips the chirality of the left-moving $\mathrm{R}$ ground state. In any case the world sheet parity operation is accompanied by one of the cyclic orbifold groups preserving $\mathcal{N}=1$ supersymmetry, which have been displayed in the tables 2.1 and 2.2 . The action of the generator $\hat{\Theta}$ in the type I picture with world sheet parity $\Omega$ is

$$
\hat{\Theta}\left(Z_{\mathrm{L}}^{i}, Z_{\mathrm{R}}^{i}\right) \hat{\Theta}^{-1}=\left(e^{-i \varphi^{(i)}} Z_{\mathrm{L}}^{i}, e^{i \varphi^{(i)}} Z_{\mathrm{R}}^{i}\right) .
$$

That of $\Theta$ is

$$
\Theta\left(Z_{\mathrm{L}}^{i}, Z_{\mathrm{R}}^{i}\right) \Theta^{-1}=\left(e^{i \varphi^{(i)}} Z_{\mathrm{L}}^{i}, e^{i \varphi^{(i)}} Z_{\mathrm{R}}^{i}\right)
$$

in the type $\mathrm{I}^{\prime}$ picture with $\Omega \mathcal{R}, \mathcal{R}$ the refection of all the $x_{(i)}^{2}$. The angles are defined via the entries $v_{i}$ of table 2.1 and 2.2

$$
\varphi^{(i)}=2 \pi v_{i}
$$

In the same way as for the bosons the complexified fermionic coordinates diagonalize $\Theta$. Both for the RR and any $N / 2$-twisted NSNS sector the operation of $\mathcal{R}$ and $\Theta$ on fermionic ground states is given by (3.43) and (2.22). If the ground state is not a spinor of the entire light-cone gauge $S O(8)$ little group, but only of a $S O(2 k)$ subgroup, one can formally set the respective $s_{i}$ in (3.43) to zero to obtain the correct transformation. There is a subtlety concerning the GSO projection in the twisted sectors when performing the T-duality. One needs to use the world sheet fermion number operators (3.44) to project on states satisfying $(-1)^{F_{L}}=(-1)^{F_{R}}=-1$. In the untwisted sector of the orientifolds this is apparently equivalent to the usual type IIA GSO projection and in the twisted sectors it guarantees that $\Omega \mathcal{R}$ is really a symmetry of the resulting theory. In the various open string sectors the GSO projection is always determined by supersymmetry.

Concretely, we shall consider the orbifold groups $\mathbb{Z}_{3}, \mathbb{Z}_{4}, \mathbb{Z}_{6}$ and $\mathbb{Z}_{6}^{\prime}$. The most simple case $\mathbb{Z}_{2}$ is not eligible because there is no distinction between a symmetric or an asymmetric reflection. In this sense it has already been covered completely by chapter 3.5. 
While it has been established for ordinary fourdimensional type I vacua that there exists no solution to the tadpole cancellation conditions for the cases $\mathbb{Z}_{4}, \mathbb{Z}_{8}, \mathbb{Z}_{8}^{\prime}$ and $\mathbb{Z}_{12}$, we obtain perturbatively consistent solutions for any of the above $\mathbb{Z}_{N}$ model. In [112] also a number of $\mathbb{Z}_{N} \times \mathbb{Z}_{M}$ orientifolds has been considered.

To define the tori one needs to use lattices that allow a crystallographic action of the orbifold groups. It is more convenient to construct the T-dual version of tori with asymmetric symmetries under $\hat{\Theta}$ in terms of tori symmetric under $\Theta$ and $\mathcal{R}$ simultaneously. The requirement of a $\Theta$ symmetry forces the angles $\theta$ which define the complex structures $U^{(i)}$ as in (2.78) to be multiples of $2 \pi / N$ after scaling all basis vectors $\mathbf{e}_{i}$ to unit length. Up to overall scales in each $\mathbb{T}^{2}$ factor, for the $\mathbb{Z}_{3}$ and $\mathbb{Z}_{6}$ case we choose the root lattice of $S U(3)^{D}$ and for the $\mathbb{Z}_{4}$ case the root lattice of $S U(2)^{2 D}, D$ the complex dimension of the compact space. Except for the $\mathbb{Z}_{3}$ example these are not of the type explored in [51], which allow to take the coxeter element of the Lie algebra as the generator of the orbifold group and have been shown explicitly to be related to Calabi-Yau compactifications there. As the particular choice of the lattice enters at various important points into the calculation of the tadpole cancellation conditions and the spectrum, different lattices may lead to even more inequivalent models. In fact, not only the choice of the orbifold group leads to a distinction of different inequivalent models but also the orientation of the lattice with respect to the operation of $\mathcal{R}$. This represents a discrete modulus of the type $\mathrm{I}^{\prime}$ theory which is directly related to the discrete NSNS $B$-field of type I [113]. Therefore, it also involves a reduction of the rank of the gauge groups and switches the symmetrization prescriptions for matter fields, i.e. their representation.

In the following we first describe in some detail the general features that arise in computing the three contributions to the massless tadpoles arising in the Klein bottle, annulus and Möbius strip amplitudes. The technical details and the explicit expressions can be found in appendix $\mathrm{C}$.

\subsubsection{Closed strings}

In the closed string sector the states with excitations of left-moving oscillators combine with their complex conjugate images on the right-moving side into $\Omega$ invariant states

$$
\begin{aligned}
& \Omega\left(\alpha_{n+k v_{i}}^{i} \tilde{\alpha}_{n+k v_{i}}^{i}\right) \Omega^{-1}=\left(\alpha_{n+k v_{i}}^{i} \tilde{\alpha}_{n+k v_{i}}^{i}\right), \\
& \Omega\left(\bar{\alpha}_{n-k v_{i}}^{i} \tilde{\bar{\alpha}}_{n-k v_{i}}^{i}\right) \Omega^{-1}=\left(\bar{\alpha}_{n-k v_{i}}^{i} \tilde{\bar{\alpha}}_{n-k v_{i}}^{i}\right),
\end{aligned}
$$

here for a field in the $k$-twisted sector. In ordinary symmetric orientifolds $\Omega$ exchanges fields of the $k$ - and $(N-k)$-twisted sectors. By (4.5) this is no longer the case and the loop channel of the Klein bottle has contributions from all twisted sectors not only from the untwisted and $N / 2$-twisted. As well opposed to standard orientifolds, the $\Omega$ invariant states are also invariant under the $\hat{\Theta}$ action

$$
\begin{aligned}
& \hat{\Theta}\left(\alpha_{n+k v_{i}}^{i} \tilde{\alpha}_{n+k v_{i}}^{i}\right) \hat{\Theta}^{-1}=\left(\alpha_{n+k v_{i}}^{i} \tilde{\alpha}_{n+k v_{i}}^{i}\right), \\
& \hat{\Theta}\left(\bar{\alpha}_{n-k v_{i}}^{i} \tilde{\bar{\alpha}}_{n-k v_{i}}^{i}\right) \hat{\Theta}^{-1}=\left(\bar{\alpha}_{n-k v_{i}}^{i} \tilde{\bar{\alpha}}_{n-k v_{i}}^{i}\right) .
\end{aligned}
$$


The important point to notice is that all insertions $\Omega \hat{\Theta}^{k}$ in the loop channel Klein bottle amplitude

$$
\mathcal{K}=\int_{0}^{\infty} \frac{d t}{t} \operatorname{Tr}\left(\frac{\Omega}{2} \mathcal{P}_{\mathrm{orb}} \mathcal{P}_{\mathrm{GSO}} e^{-2 \pi t\left(L_{0}+\bar{L}_{0}\right)}\right)
$$

produce the same contribution, just canceling the $1 / N$ normalization of the orbifold projector. The relation

$$
\Omega \hat{\Theta}^{k}=\hat{\Theta}^{N-k} \Omega
$$

implies that in the tree channel only untwisted closed string states propagate between the two cross-caps on both sides of the tube. Thus, world sheet consistency requires that transforming the loop channel amplitude (4.7) with all sectors contributing into the tree channel leads to an amplitude in which only $\mathbb{Z}_{N}$ invariant states from the untwisted sector contribute. In doing the computation one realizes that this world-sheet consistency condition is not always satisfied automatically. For some orientations of the lattice that defines the torus, the various terms in the tree channel do not come with the correct normalization. Therefore, the completion of the $\mathbb{Z}_{N}$ projector in the tree channel will serve as the guiding principle in constructing consistent models.

Since the action of $\Omega \hat{\Theta}^{k}$ is trivial on the oscillator part, the complete Klein bottle amplitude factorizes into a trace over the oscillators times a trace over the bosonic zeromodes in each $\mathbb{Z}_{N}$ twisted sector. To compute the latter contribution in the loop channel, one needs to determine the winding and KK modes which are invariant under the operator appearing in the trace. To begin with, let us consider a single twodimensional torus, on which $\Theta$ acts as a rotation by an angle $\varphi=2 \pi / N$. Let us define the lattice type $\mathbf{A}$ to be the orientation of the $\mathbb{Z}_{N}$ lattice such that the reflection $\mathcal{R}$ acts orthogonally to one of the two basis vectors $\mathbf{e}_{i}$ that span the lattice. In this case the dual relation

$$
\left(\Omega \hat{\Theta}^{k}\right) \hat{\Theta}^{2}=\hat{\Theta}^{-1}\left(\Omega \hat{\Theta}^{k}\right) \hat{\Theta}
$$

implies that the partition function of the lattice with $\Omega \hat{\Theta}^{k}$ insertion does only depend on $k$ being even or odd. Rotating the lattice by an angle of $\pi / N$ for even and $\pi /(2 N)$ for odd $N$ leads to the lattice type $\mathbf{B}$, the only non-trivial rotation maintaining a crystallographic action of $\mathcal{R}$. Due to the relation

$$
\hat{\Theta}^{-1 / 2}\left(\Omega \hat{\Theta}^{k}\right) \hat{\Theta}^{1 / 2}=\left(\Omega \hat{\Theta}^{k}\right) \hat{\Theta}
$$

the two values for $k$, even and odd, get exchanged. Among modifying the other moduli this switch amounts to changing the background NSNS $B$-field in the asymmetric "flux" picture. In figure 4.1 the distinction has been depicted for a lattice isomorphic to the $S U(3)$ root lattice. The fixed points of $\Theta$ are indicated by circles.

In orbifolds where $N$ is a power of 2 , one can choose the $\mathbf{A}$ type lattice orthogonal, i.e. with vanishing dual $B$-field. Therefore the rank of the gauge groups for the $\mathbf{B}$ models is reduced by factors of 2 . While we have been presenting examples of models without the strict reduction by $2^{-\mathrm{rk}(B) / 2}$ in chapter 3.5 the models at hand do obey this rule. For 


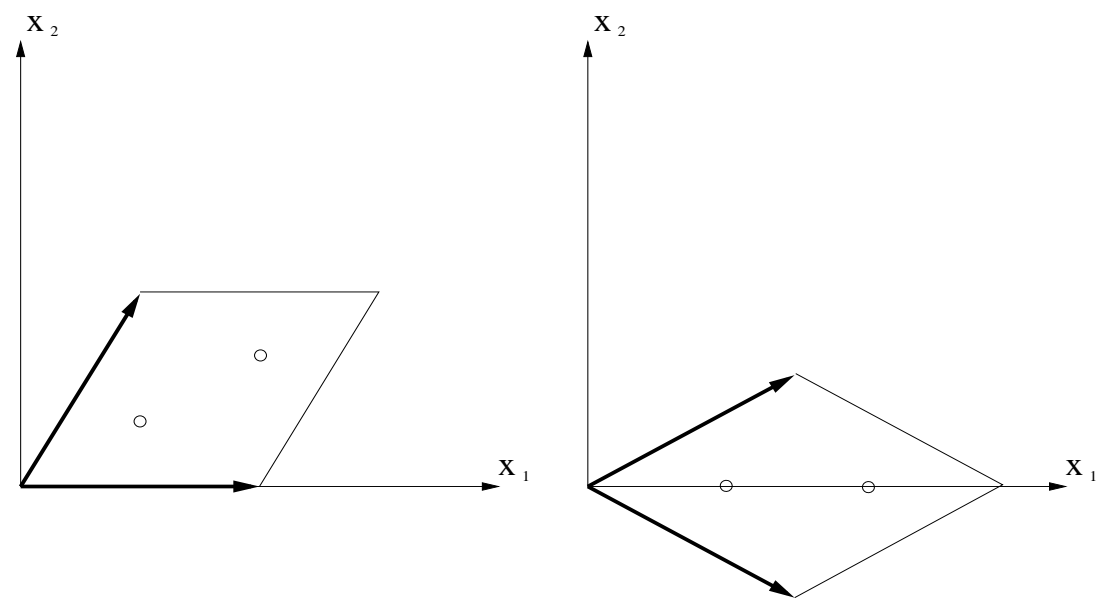

Figure 4.1: $\mathbf{A}$ and $\mathbf{B}$ type lattices with $\mathbb{Z}_{3}$ symmetry

lattices which also require a symmetry under rotations of odd order, imposed whenever the orbifold group has subgroups $\mathbb{Z}_{M}$ with odd $M$, both, the $\mathbf{A}$ and the $\mathbf{B}$ type lattices lead to a maximal rank reduction. Note, that in the special case of the $\mathbb{Z}_{3}$ orbifold the rotation $\Theta^{1 / 2}$ is a symmetry of the $S U(3)$ root lattice implying that the Klein bottle contributions are entirely independent of $k$.

It appears to be the correct choice for world sheet consistency, that whenever the orbifold model has two complex directions with $N v_{i}$ odd and in addition one with $N v_{i}$ even, $\mathcal{R}$ must act differently on the two odd directions and arbitrarily on the even direction. In other words, we choose lattices of type $\mathbf{A B}$ or $\mathbf{B A}$ in six dimensions and may choose either $\mathbf{A B}$ or $\mathbf{B A}$ for the two odd directions and $\mathbf{A}$ or $\mathbf{B}$ for the even in four dimensions. This gives a rather large variety of inequivalent models. In this way one produces a partition function which in the untwisted sector loop channel yields identical contributions for all $\hat{\Theta}^{k}$ insertions in the trace. Accidentally, this remains true for the twisted sectors with the exception of the $\mathbb{Z}_{6}^{\prime}$ orientifold. In the $\mathbb{Z}_{6}^{\prime}$ case, the two choices $\mathbf{A B}$ and $\mathbf{B A}$ for the two odd tori lead to different models. Summarizing, in six dimensions we have three inequivalent models $\{\mathbf{A A}, \mathbf{A B}, \mathbf{B B}\}$ for the $\mathbb{Z}_{3}$ and only a single choice $\mathbf{A B}$ for the $\mathbb{Z}_{4}$ and $\mathbb{Z}_{6}$. In four dimensions for the $\mathbb{Z}_{3}$ orbifold we have the four inequivalent choices $\{\mathbf{A A A}, \mathbf{A A B}, \mathbf{A B B}, \mathbf{B B B}\}$, for the $\mathbb{Z}_{4}$ and the $\mathbb{Z}_{6}$ the two choices $\{\mathbf{A B A}, \mathbf{A B B}\}$ and finally for the $\mathbb{Z}_{6}^{\prime}$ the four choices $\{\mathbf{A A B}, \mathbf{A B B}, \mathbf{B A A}, \mathbf{B B A}\}$. All other combinations have been inspected and found not to respect the open-closed consistency condition, i.e. they do not produce orbifold invariant cross-caps in the tree channel.

In order to compute the loop channel Klein bottle amplitude in the $l$-twisted sector, we have to determine which of the fixed points of $\Theta^{l}$ are invariant under the action of $\mathcal{R} \Theta^{k}$ in the T-dual picture. They enter the loop channel amplitude as extra multiplicity again and can also be determined by the analogue of the CFT computation as performed in section 2.4.4. With the exception of the $\mathbb{Z}_{6}^{\prime}$ orientifold the number of invariant fixed points fortunately does not depend on $k$, though different individual points are invariant. These fixed point multiplicities nontrivially conspire with the relative factors arising 
from the modular transformation of the lattice contributions in order to complete the tree channel projector.

Regarding these subtleties the computation of the various amplitudes is a straightforward though still tedious task. All the results for the different traces involved can be found in appendix $\mathrm{C}$ and the $\mathbb{Z}_{4}$ prototype will be discussed in greater detail in the following section.

Finally, we need to find the massless spectra. In the untwisted sector $\hat{\Theta}$ invariant leftand right-moving massless states have to be symmetrized and antisymmetrized under $\Omega$ in the NSNS and the RR sector respectively. This always contributes the graviton and dilaton from the NSNS sector and some model dependent number of additional neutral hyper and chiral multiplets. For the twisted sectors the ground state energies are again given by applying (3.83) and one carefully needs to inspect the transformation properties of the fixed points. Those which are invariant under $\mathcal{R}$ and $\Theta$ need to be properly symmetrized and antisymmetrized as in the untwisted sector. Fixed points which are only invariant under a combination of $\mathcal{R}$ and $\Theta$ or even under neither of the two transformations require less or no symmetrization. Following this procedure, the total number of neutral hyper- and tensormultiplets in six dimensions are found to add up to 21, while the number chiral and vectormultiplets in four dimensions ${ }^{1}$ is exactly the sum of the Hodge numbers $h^{(1,1)}$ and $h^{(2,1)}$ of the blown up toroidal orbifold, the number of complex structure and Kähler deformations of the corresponding Calabi-Yau manifold [51]. In particular, for inequivalent orientifold models with identical orbifold groups we find the same net number of multiplets in the closed string spectrum, while the individual states are different. In table 4.1 we display the closed string spectra of hyper and tensor multiplets for sixdimensional models

\begin{tabular}{|c|c|c|c|c|c|}
\hline $\begin{array}{c}\text { Orbifold } \\
\text { group }\end{array}$ & Model & Untwisted & $\begin{array}{c}\Theta, \Theta^{-1} \\
\text { twisted }\end{array}$ & $\begin{array}{c}\Theta^{2}, \Theta^{-2} \\
\text { twisted }\end{array}$ & $\begin{array}{c}\Theta^{3} \\
\text { twisted }\end{array}$ \\
\hline \hline $\mathbb{Z}_{3}$ & AA & $3 H$ & $10 H+8 T$ & absent & absent \\
$\mathbb{Z}_{3}$ & AB & $3 H$ & $12 H+6 T$ & absent & absent \\
$\mathbb{Z}_{3}$ & BB & $3 H$ & $18 H$ & absent & absent \\
$\mathbb{Z}_{4}$ & AB & $3 H$ & $8 H$ & $9 H+1 T$ & absent \\
$\mathbb{Z}_{6}$ & AB & $3 H$ & $2 H$ & $8 H+2 T$ & $5 H+1 T$ \\
\hline
\end{tabular}

Table 4.1: Closed string spectra in $d=6$

\footnotetext{
${ }^{1}$ We would like to acknowledge that [114] has drawn our attention to the necessity of further distinguishing states in the twisted RR sectors, that transform as scalars respectively as vectors.
} 
and in table 4.2 those for fourdimensional orientifolds.

\begin{tabular}{|c|c|c|c|c|c|}
\hline $\begin{array}{c}\text { Orbifold } \\
\text { group }\end{array}$ & Model & Untwisted & $\begin{array}{c}\Theta, \Theta^{-1} \\
\text { twisted }\end{array}$ & $\begin{array}{c}\Theta^{2}, \Theta^{-2} \\
\text { twisted }\end{array}$ & $\begin{array}{c}\Theta^{3} \\
\text { twisted }\end{array}$ \\
\hline \hline $\mathbb{Z}_{3}$ & AAA & $9 C$ & $14 C+13 V$ & absent & absent \\
$\mathbb{Z}_{3}$ & AAB & $9 C$ & $15 C+12 V$ & absent & absent \\
$\mathbb{Z}_{3}$ & ABB & $9 C$ & $18 C+9 V$ & absent & absent \\
$\mathbb{Z}_{3}$ & BBB & $9 C$ & $27 C$ & absent & absent \\
$\mathbb{Z}_{4}$ & ABA & $6 C$ & $16 C$ & $15 C+1 V$ & absent \\
$\mathbb{Z}_{4}$ & ABB & $6 C$ & $12 C+4 V$ & $15 C+1 V$ & absent \\
$\mathbb{Z}_{6}$ & ABA & $5 C$ & $2 C+1 V$ & $9 C+6 V$ & $10 C+1 V$ \\
$\mathbb{Z}_{6}$ & ABB & $5 C$ & $3 C$ & $12 C+3 V$ & $10 C+1 V$ \\
$\mathbb{Z}_{6}^{\prime}$ & AAB & $4 C$ & $7 C+5 V$ & $14 C+4 V$ & $10 C+2 V$ \\
$\mathbb{Z}_{6}^{\prime}$ & ABB & $4 C$ & $9 C+3 V$ & $18 C$ & $10 C+2 V$ \\
\hline
\end{tabular}

Table 4.2: Closed string spectra in $d=4$

In the untwisted sector there are always the graviton and the dilaton multiplet in addition to the chiral multiplets as given in the table.

\subsubsection{Open strings}

The more general features of the open string sector can nearly be guessed from the symmetry properties of the models at hand. We know that the untwisted sector requires a set of 32 D9-branes in the absence of any $B$-flux. From the nontrivial complex structures of the tori as required by the symmetry under $\Theta$ in the T-dual picture one can just read off the rank of the dual $B$-field. It is always maximal for $N=3,6,6^{\prime}$ and for $N=4$ any $\mathbf{B}$ type lattice raises the rank of $B$ by two. The resulting set of D9-branes needs to be invariant under $\hat{\Theta}$ and we have to consider sets of D9-branes with magnetic fluxes that correspond to asymmetric rotations by angles $2 \pi / N$. For even $N$ the spectrum will also contain branes with infinite flux, D5-branes. Of course, we also have to include the D9-branes with opposite flux $-2 \pi / N$. In the T-dual picture all this translates into arrays of $\mathrm{D}(9-D)$-branes on a torus of complex dimension $D$, arranged at angles $2 \pi / N$ and symmetric with respect to a reflection at the $x_{(i)}^{2}$ axes. The mode expansions of the open strings stretching between two such D-branes qualitatively match those of the fields in the closed string twisted sectors, open strings stretching between D-branes at relative angle $\pi v_{i}$ carry fields with modings shifted by $v_{i}$. Actually, we shall only consider arrays of D9-branes where one set consists of D9-branes without any magnetic flux and the rest is generated by applying $\hat{\Theta}^{1 / 2}$ successively. Different configurations may be considered but we have frequently encountered problems with the open-closed consistency when straying apart from the above rule. 
In the open string sector one has to compute the annulus

$$
\mathcal{A}=\int_{0}^{\infty} \frac{d t}{t} \operatorname{Tr}\left(\mathcal{P}_{\text {orb }} \mathcal{P}_{\mathrm{GSO}} e^{-2 \pi t L_{0}}\right)
$$

and the Möbius strip amplitude

$$
\mathcal{M}= \pm \int_{0}^{\infty} \frac{d t}{t} \operatorname{Tr}\left(\mathcal{P}_{\mathrm{orb}} \mathcal{P}_{\mathrm{GSO}} e^{-2 \pi t L_{0}}\right)
$$

These amplitudes only receive a nonvanishing contribution from open strings in the $(\mu, \mu+n)$ sector, if the action of the operator in the trace leaves the two branes and the orientation of the open string invariant. If the moding of the fields in the $(\mu, \mu+n)$ open string sector is identical to that of the fields in the closed string sector twisted by $\hat{\Theta}^{k}$, we also use the term " $k$-twisted" for the open string sector. For even $N$ the operator $\hat{\Theta}^{N / 2}$ leaves all $\mathrm{D} 9_{\mu}$-branes invariant and has a nontrivial action on the Chan-Paton factors as usually described by a matrix $\gamma_{(N / 2) \mu}$. Correspondingly, the annulus amplitude gives rise to an additional $N / 2$-twisted sector tadpole, which is absent in the Klein bottle and Möbius strip.

Some care has to be taken when computing the contributions of momenta and winding states, which are present for $(\mu, \mu+n)$ strings whenever the two branes have identical flux on any $\mathbb{T}^{2}$ and the operator in the trace acts trivially there. The mass spectrum is then given as usually by (2.92). In the Möbius strip amplitude these KK and winding modes also have to be invariant under $\Omega$. In the presence of a nonvanishing NSNS $B$-field (3.106) leads to a doubling of winding states as compared to the annulus. The present choice of the tori and branes guarantees that the contributions of the zero-modes are independent of the operator in the trace for a given sector, except for the $(\mu, \mu+2)$ strings in the $\mathbb{Z}_{6}^{\prime}$.

A very important point in the computation are the extra intersection multiplicities of some twisted open string sectors. For computing the annulus and Möbius strip amplitude, the multiplicity of such intersection points needs to be reduced to those invariant under the operator in the trace. As an example we have depicted the D-brane configurations of the $\mathbf{A}$ and $\mathbf{B}$ type $S U(3)$ root lattices with their respective intersections in figure 4.2. Obviously, the KK momenta given by the the length of the branes and the number of intersection points depend on the type of lattice. It turns out that this number of invariant intersection points of $(\mu, \mu+n)$ branes is independent of $\mu$ with the exception of $n=2$ in the $\mathbb{Z}_{6}^{\prime}$ orientifold. In [113] these extra multiplicities have been related via T-duality to extra factors for twisted sectors in ordinary $\Omega$ orientifolds with background $B$-field. So far we have presented all the novel ingredients needed to compute the two open string amplitudes and the details are left to the appendix $\mathrm{C}$ and to the discussion of the $\mathbb{Z}_{4}$ example.

Let us now discuss the main steps for computing the massless open string spectrum. One has to be very careful again with the contributions of the different intersection points. First of all we notice, that one always gets a tadpole cancellation condition of the form

$$
\left(N_{\mu}-2^{5-\mathrm{rk}(B) / 2}\right)^{2}=0
$$




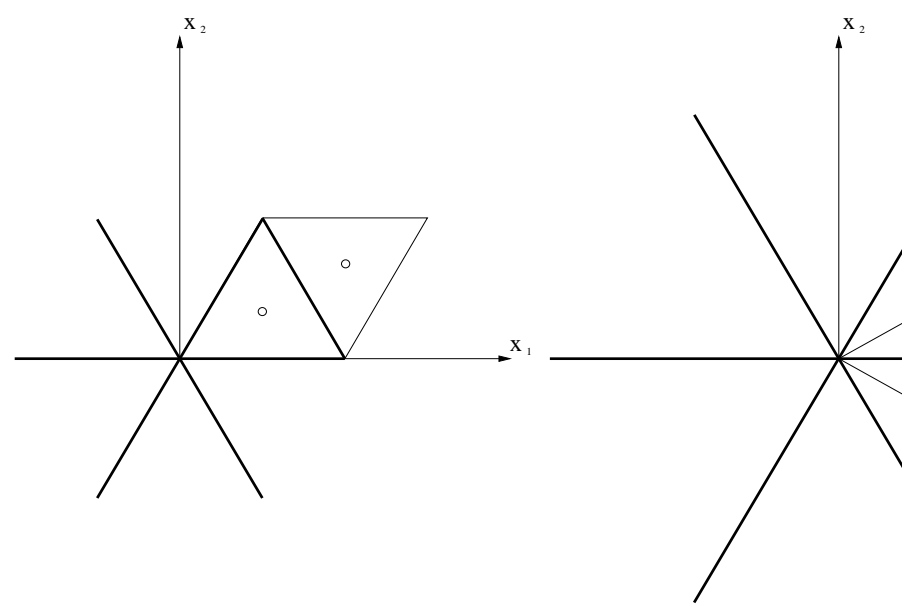

Figure 4.2: D-branes wrapping $\mathbf{A}$ and $\mathbf{B}$ type lattices

fixing the number $N_{\mu}$ of $\mathrm{D} 9_{\mu}$-branes of each type. For even $\mathbb{Z}_{N}$ there is an additional $N / 2$-twisted tadpole condition, which requires

$$
\operatorname{tr}\left(\gamma_{(N / 2) \mu}\right)=0
$$

exactly resembling the computation for the standard sixdimensional $\mathbb{Z}_{2}$ orientifold discussed in [80]. We can therefore copy their solution for the Chan-Paton degrees of freedom, which implies an $S O\left(N_{\mu}\right)$ gauge group on each stack of branes, broken to its $U\left(N_{\mu} / 2\right)$ subgroup, if $N$ is even. We simplify the analysis by looking at those open strings only, which begin on one of the branes located in the fixed plane of $\mathcal{R}$, as all other states are related to these by some action of the orientifold group. Only for the $\mathbb{Z}_{6}^{\prime}$ model the situation is slightly different and one has to consider the branes of odd and even $\mu$ separately.

Similar to the closed string case, for the twisted sectors things become more involved. First one needs to do the same distinction concerning the Chan-Paton labels as for the untwisted sector above. Moreover, one has to distinguish the contributions of the various intersection points, which may be invariant under $\Theta$ and $\mathcal{R}$ or not. Finally, in four dimensions one must keep track of various phase factors, that appear via modular transformation and provide additional relative signs. This needs to be taken into account, when doing any symmetrization or antisymmetrization of Chan-Paton labels. Since the computation of the open string spectrum of the fourdimensional asymmetric orientifolds at hand is similar to a $\mathbb{Z}_{2}$ standard orientifold, we only find nonchiral spectra in rather small gauge groups. Remember, that standard fourdimensional $\mathbb{Z}_{N}$ orientifolds for $N>2$ generically have chiral spectra whenever solutions exist.

After having explained all the principles, we now come to the detailed discussion of one example. We have chosen the $\mathbb{Z}_{4}$ as it already exhibits all the generic features we have went through while not being as complicated as others in technical terms. 


\subsection{Sixdimensional models}

The sixdimensional $\mathbb{Z}_{4}$ orientifold is defined by $v_{i}=(1 / 4,-1 / 4)$. By the general arguments given above we expect a contribution to the untwisted tadpole which requires D9and D5-branes to cancel it, both being mapped upon each other by $\hat{\Theta}$. Only the annulus amplitude will have a contribution from the 2-twisted sector which is implemented via a projection on the Chan-Paton factors that breaks the orthogonal gauge group to its unitary subgroup. Its rank will be determined by the rank of the $B$-field. The $\mathbf{A}$ and $\mathbf{B}$ configuration of lattices that define the $\mathbb{T}^{4}$ are depicted in figure 4.3. As mentioned, another choice does not lead to a consistent matching of the open and closed string spectra in the orientifold.
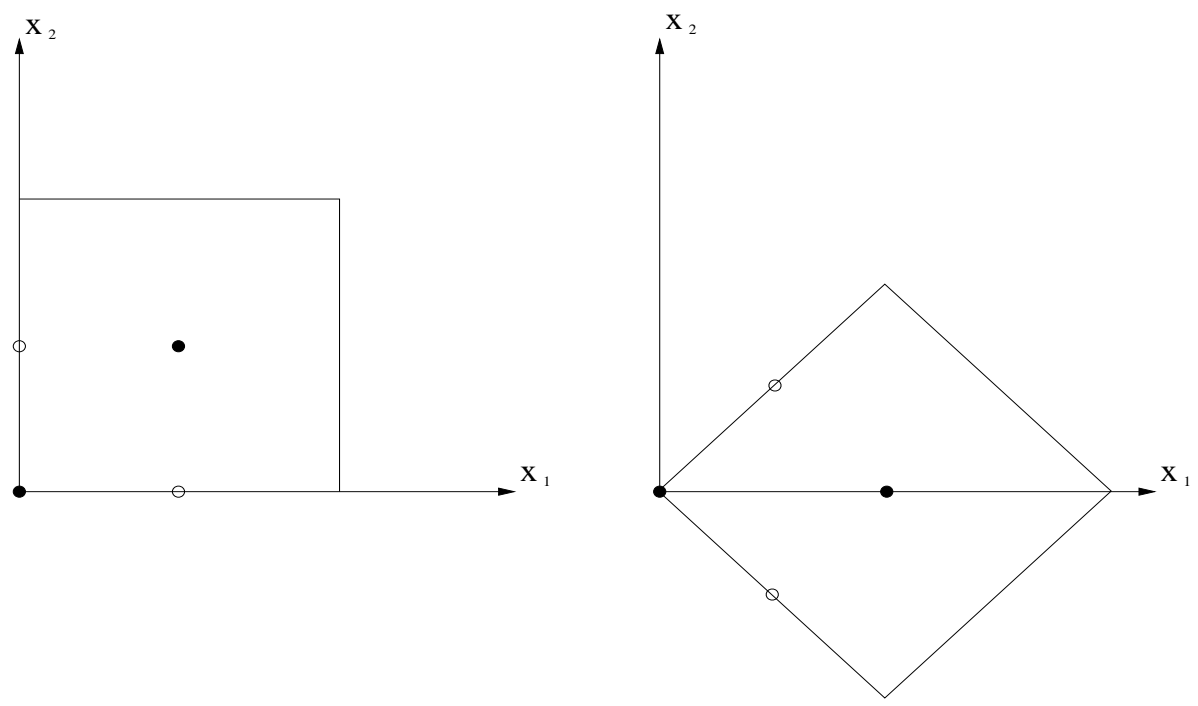

Figure 4.3: $\mathbf{A}$ and $\mathbf{B}$ type lattices with $\mathbb{Z}_{4}$ symmetry

The KK and winding states invariant under $\Omega \mathcal{R} \Theta^{2 k}$ can easily be deduced from the geometry of figure 4.3 as

$$
p^{\mathbf{A}}=\frac{m}{R}, \quad l^{\mathbf{A}}=n R, \quad p^{\mathbf{B}}=\sqrt{2} \frac{m}{R}, \quad l^{\mathbf{B}}=\sqrt{2} n R,
$$

for the respective orientations of the lattice. The states invariant under $\Omega \mathcal{R} \Theta^{2 k+1}$ are obtained by exchanging $\mathbf{A}$ and $\mathbf{B}$ in (4.15). By the open-closed consistency we are forced to choose the $\mathbb{T}^{4}$ of $\mathbf{A B}$ type. This is so far a heuristic observation with no fundamental explanation. The fixed points of $\Theta$ are indicated in figure 4.3 by black circles and additionally those of $\Theta^{2}$ by white circles. It is evident that all four $\Theta$ fixed points are also invariant under $\mathcal{R}$ and that only eight of the sixteen fixed points of $\Theta^{2}$ are invariant likewise. Thus, the $\hat{\Theta}$ and $\hat{\Theta}^{3}$ twisted sector contribution to the Klein bottle amplitude is weighted by a factor of four and the $\hat{\Theta}^{2}$ twisted sector contribution is weighted by a factor of eight. The actual computation of the oscillator sums is summarized using the formulas given in appendix $\mathrm{C}$ and we restrict ourselves to writing out the tree channel 
amplitude again. It comes out to be given by

$$
\begin{aligned}
\tilde{\mathcal{K}}=2^{6} c_{D}(1-1) \int_{0}^{\infty} d l\left(\tilde{\mathcal{K}}_{\left(\frac{1}{4},-\frac{1}{4}\right)}^{(0)} \tilde{\mathcal{L}}[4,4] \tilde{\mathcal{L}}[2,2]+2 \tilde{\mathcal{K}}_{\left(\frac{1}{4},-\frac{1}{4}\right)}^{(1)}\right. \\
\left.+4 \tilde{\mathcal{K}}_{\left(\frac{1}{4},-\frac{1}{4}\right)}^{(2)}+2 \tilde{\mathcal{K}}_{\left(\frac{1}{4},-\frac{1}{4}\right)}^{(3)}\right) .
\end{aligned}
$$

The third term in (4.16) from the 2-twisted sector is zero. Nevertheless, it is useful to keep it, as its coefficient still demonstrates the appearance of the complete projector with numerical prefactors $4 \sin ^{2}(\pi k / N)$ in front of the $\vartheta$-functions. The next step is to introduce appropriate D7-branes to cancel the tadpoles, which is done in the generic fashion described above. There are two sets of D7-branes, the even and odd ones, which are not related to each other by the rotation $\Theta$ but only by $\Theta^{1 / 2}$. Thus, we expect them to support two independent factors of a product gauge group. By symmetry we also assume that the action of $\hat{\Theta}^{2}$ on the Chan-Paton indices is equal for all the individual D7-branes and we abbreviate

$$
\operatorname{tr}\left(\gamma_{2 \mu}\right)=\gamma_{2}
$$

Further one needs to regard the extra multiplicity for the 2-twisted sector which stems from the invariant intersection of the respective branes. The tree channel finally reads

$$
\begin{aligned}
\tilde{\mathcal{A}}=2^{-2} c_{D}(1-1) \int_{0}^{\infty} & d l\left(N_{\mu}^{2} \tilde{\mathcal{A}}_{\left(\frac{1}{4},-\frac{1}{4}\right)}^{(0,0)} \tilde{\mathcal{L}}[1,1] \tilde{\mathcal{L}}[2,2]+8 \gamma_{2}^{2} \tilde{\mathcal{A}}_{\left(\frac{1}{4},-\frac{1}{4}\right)}^{(0,2)}\right. \\
+ & 2 N_{\mu}^{2} \tilde{\mathcal{A}}_{\left(\frac{1}{4},-\frac{1}{4}\right)}^{(1,0)}+2 \gamma_{2}^{2} \tilde{\mathcal{A}}_{\left(\frac{1}{4},-\frac{1}{4}\right)}^{(1,2)}+4 N_{\mu}^{2} \tilde{\mathcal{A}}_{\left(\frac{1}{4},-\frac{1}{4}\right)}^{(2,0)}+4 \gamma_{2}^{2} \tilde{\mathcal{A}}_{\left(\frac{1}{4},-\frac{1}{4}\right)}^{(2,2)} \\
+ & \left.2 N_{\mu}^{2} \tilde{\mathcal{A}}_{\left(\frac{1}{4},-\frac{1}{4}\right)}^{(3,0)}+2 \gamma_{2}^{2} \tilde{\mathcal{A}}_{\left(\frac{1}{4},-\frac{1}{4}\right)}^{(3,2)}\right) .
\end{aligned}
$$

Again not all sectors produce nonvanishing contributions but have been listed to demonstrate the correct normalization. One can also perform an analysis of the twisted tadpoles on an individual basis treating all $\mathrm{D} 7_{\mu}$-branes separately. This proves that one needs to have all the $\gamma_{2 \mu}$ matrices traceless and no nontrivial compensation of anomaly-inflows can take place. The degeneration of the twisted sectors has to be taken into account in the Möbius amplitude as well. For the tree channel Möbius amplitude the result is

$$
\begin{aligned}
\tilde{\mathcal{M}}=-2^{3} N_{\mu} c_{D}(1-1) \int_{0}^{\infty} d l\left(\tilde{\mathcal{M}}_{\left(\frac{1}{4},-\frac{1}{4}\right)}^{(0,0)} \tilde{\mathcal{L}}[4,4] \tilde{\mathcal{L}}[8,2]+\tilde{\mathcal{M}}_{\left(\frac{1}{4},-\frac{1}{4}\right)}^{(0,2)}\right. \\
\left.+4 \tilde{\mathcal{M}}_{\left(\frac{1}{4},-\frac{1}{4}\right)}^{(2,1)}+4 \tilde{\mathcal{M}}_{\left(\frac{1}{4},-\frac{1}{4}\right)}^{(2,3)}\right) .
\end{aligned}
$$

Adding up all tree channel amplitudes we derive two tadpole cancellation conditions

$$
\frac{1}{4}\left(N_{\mu}-16\right)^{2}=0, \quad \operatorname{tr}\left(\gamma_{2 \mu}\right)^{2}=0,
$$

yielding $N_{\mu}=16 \mathrm{D} 7_{\mu}$-branes of each type. Thus we have a gauge group $U(8) \times U(8)$, one factor from the even branes another from the odd ones and both with the same projection on the gauge bundle. The open string spectrum consists of the untwisted $(\mu, \mu)$ sector with a vectormultiplet in the adjoint and two hypermultiplets in the antisymmetric 
representation for each gauge factor separately. The sectors $(\mu, \mu+1)$ and $(\mu, \mu+3)$ contribute two hypermultiplets in the bifundamental representation and finally the $(\mu, \mu+2)$ sector yields another two hypermultiplets in the antisymmetric representation for each factor. This takes the extra intersection number into account. Summarizing we get the spectrum of table 4.3 .

The analogous computation for the $\mathbb{Z}_{3}$ and $\mathbb{Z}_{6}$ orbifold groups of table 2.1 proceeds in a very similar manner in principle. A particular incident of the $\mathbb{Z}_{3}$ model is that the partition function of the bosonic zero-modes is entirely independent of the $\mathbf{A}$ or $\mathbf{B}$ type of the lattice, such there is a distinction of three inequivalent possibilities. The result is an $S O(8)$ gauge group for $\mathbb{Z}_{3}$ and $U(4) \times U(4)$ for $\mathbb{Z}_{6}$ both having necessarily $\operatorname{rk}(B)=4$. The open string spectra are displayed in table 4.3 together with those of the $\mathbb{Z}_{4}$, where all the charged hypermultiplets have been listed.

\begin{tabular}{|c|c|c|c|c|}
\hline Model & $(\mu, \mu)$ & $\begin{array}{c}(\mu, \mu+1) \\
+(\mu, \mu+N-1)\end{array}$ & $\begin{array}{c}(\mu, \mu+2) \\
+(\mu, \mu+N-2)\end{array}$ & $(\mu, \mu+3)$ \\
\hline $\begin{array}{c}\mathbb{Z}_{3} \mathbf{A A} \\
S O(8)\end{array}$ & $(28)$ & $(28)$ & absent & absent \\
\hline $\begin{array}{c}\mathbb{Z}_{3} \mathbf{A B} \\
S O(8)\end{array}$ & $(28)$ & $3(\mathbf{2 8})$ & absent & absent \\
\hline $\begin{array}{c}\mathbb{Z}_{3} \text { BB } \\
S O(8)\end{array}$ & $(28)$ & $9(\mathbf{2 8})$ & absent & absent \\
\hline $\begin{array}{c}\mathbb{Z}_{4} \mathbf{A B} \\
U(8) \times U(8)\end{array}$ & $2(\mathbf{1}, \mathbf{2 8}) \oplus 2(\mathbf{2 8}, \mathbf{1})$ & $2(\mathbf{8}, \mathbf{8})$ & $2(\mathbf{1}, \mathbf{2 8}) \oplus 2(\mathbf{2 8}, \mathbf{1})$ & absent \\
\hline $\begin{array}{c}\mathbb{Z}_{6} \mathbf{A B} \\
U(4) \times U(4)\end{array}$ & $2(\mathbf{1}, \mathbf{6}) \oplus 2(\mathbf{6}, \mathbf{1})$ & $2(\mathbf{4}, \mathbf{4})$ & $\begin{array}{c}4(\mathbf{1}, \mathbf{6}) \oplus 4(\mathbf{6}, \mathbf{1}) \\
\oplus(\mathbf{1}, \mathbf{a d} \mathbf{j}) \oplus(\mathbf{a d j}, \mathbf{1})\end{array}$ & $4(\mathbf{4}, \mathbf{4})$ \\
\hline
\end{tabular}

Table 4.3: Open string spectra in $d=6$

It is an easy check of the consistency of these models to test the vanishing of the irreducible gravitational and nonabelian anomalies. Due to the presence of background fluxes we also expect a generalized Green-Schwarz mechanism to be able to cancel $U(1)$ anomalies if appropriate.

\subsection{Fourdimensional models}

In four dimensions the variety of models is fairly larger due to the freedom in the orientation of the lattice with respect to $\mathcal{R}$, equivalent to a different choice of the background $B$-field in the "flux" picture. 


\subsubsection{The example $\mathbb{T}^{6} / \mathbb{Z}_{4}$}

Again we exemplify the $\mathbb{Z}_{4}$ case, given by $v=(1,1,-2) / 4$, and only mention the results for the other models. Up to rescaling for the $\mathbb{T}^{6}$ we use the root lattice of $S U(2)^{6}$. We can refer to figure 4.3 for the two possible choices of relative orientations of the $S U(2)^{2}$ lattice with respect to $\mathcal{R}$, which distinguishes the $\mathbf{A}$ and $\mathbf{B}$ type lattices. For the fourdimensional $\hat{\Theta}$ the rules for obtaining the invariant zero-mode spectrum change slightly. Given (4.15) for states invariant under $\Omega \hat{\Theta}^{2 k}$, the states invariant under $\Omega \hat{\Theta}^{2 k+1}$ are obtained by exchanging $\mathbf{A}$ and $\mathbf{B}$ in for the first two $S U(2)^{2}$ tori, whereas they are identical to (4.15) for the third. We now have to pick the $\mathbf{A B}$ type for the first two tori and we are free to choose it either $\mathbf{A}$ or $\mathbf{B}$ for the third. Thus, we get the two inequivalent models ABA and ABB. We display all results first for the ABA model and state relative factors for the $\mathbf{A B B}$ case separately. The number of fixed points in $\Theta$ and $\Theta^{3}$ twisted sectors is 16 in either case, but for $\mathbf{A B B}$ only 8 are invariant under $\mathcal{R} \Theta^{k}$. In the $\Theta^{2}$ twisted sector one has also 16 fixed points, of which one half is invariant under $\mathcal{R} \Theta^{k}$ both for $\mathbf{A B A}$ and ABB.

All ingredients needed to compute the Klein bottle trace have thus been collected, the result in the tree channel reads

$$
\begin{array}{r}
\tilde{\mathcal{K}}=2^{5} c_{D}(1-1) \int_{0}^{\infty} d l\left(\tilde{\mathcal{K}}_{\left(\frac{1}{4}, \frac{1}{4},-\frac{1}{2}\right)}^{(0)} \tilde{\mathcal{L}}[4,4]^{2} \tilde{\mathcal{L}}[2,2]+4 \tilde{\mathcal{K}}_{\left(\frac{1}{4}, \frac{1}{4},-\frac{1}{2}\right)}^{(1)}\right. \\
\left.\quad-4 \tilde{\mathcal{K}}_{\left(\frac{1}{4},-\frac{1}{4}, 0\right)}^{(2)} \tilde{\mathcal{L}}[4,4]-4 \tilde{\mathcal{K}}_{\left(\frac{1}{4}, \frac{1}{4},-\frac{1}{2}\right)}^{(3)}\right) .
\end{array}
$$

The relative factors are in perfect match with the expected ones in (C.9). This is also true for the ABB lattice, as the only difference is an overall factor of $1 / 2$, originating in conspiracy of the lattice partition functions and the reduction of the number of invariant fixed points.

To cancel the tadpole of the Klein bottle, we introduce D6 ${ }_{\mu}$-branes into the background as before. The relative angles are $\pi v_{i}$ and the $\mathrm{D} 6_{1}$-branes are lying entirely inside the fixed plane of $\mathcal{R}$ corresponding to D9-branes without magnetic flux. Again the even and odd branes carry two distinct factors of the gauge group and the only fields that transform nontrivially under both factors arise in the $(\mu, \mu+1)$ and $(\mu, \mu+3)$ open string sectors. The complete tree channel annulus amplitude reads

$$
\begin{aligned}
\tilde{\mathcal{A}}=2^{-3} c_{D}(1-1) & \int_{0}^{\infty} d l\left(N_{\mu}^{2} \tilde{\mathcal{A}}_{\left(\frac{1}{4}, \frac{1}{4},-\frac{1}{2}\right)}^{(0,0)} \tilde{\mathcal{L}}[1,1]^{2} \tilde{\mathcal{L}}[2,2]+8 \gamma_{2}^{2} \tilde{\mathcal{A}}_{\left(\frac{1}{4},-\frac{1}{4}, 0\right)}^{(0,2)} \tilde{\mathcal{L}}[1,1]\right. \\
& +4 N_{\mu}^{2} \tilde{\mathcal{A}}_{\left(\frac{1}{4}, \frac{1}{4},-\frac{1}{2}\right)}^{(1,0)}+4 \gamma_{2}^{2} \tilde{\mathcal{A}}_{\left(\frac{1}{4}, \frac{1}{4},-\frac{1}{2}\right)}^{(1,2)}-4 N_{\mu}^{2} \tilde{\mathcal{A}}_{\left(\frac{1}{4},-\frac{1}{4}, 0\right)}^{(2,0)} \tilde{\mathcal{L}}[1,1] \\
& \left.+4 \gamma_{2}^{2} \tilde{\mathcal{A}}_{\left(\frac{1}{4},-\frac{1}{4}, 0\right)}^{(2,2)} \tilde{\mathcal{L}}[1,1]-4 N_{\mu}^{2} \tilde{\mathcal{A}}_{\left(\frac{1}{4}, \frac{1}{4},-\frac{1}{2}\right)}^{(3,0)}-4 \gamma_{2}^{2} \tilde{\mathcal{A}}_{\left(\frac{1}{4}, \frac{1}{4},-\frac{1}{2}\right)}^{(3,2)}\right) .
\end{aligned}
$$

If we change the lattice to $\mathbf{A B B}$, while keeping the branes fixed, we get an extra overall factor of 2 due to different zero-mode quantization as well as a different intersection number for $(\mu, \mu+1)$ strings. In both cases the relative normalization (C.9) comes out correctly. 
The final contribution comes from the Möbius strip amplitude, where the invariant windings and momenta are independent of the particular choice of the lattice:

$$
\begin{aligned}
\tilde{\mathcal{M}}=-2^{2} N_{\mu} c_{D}(1-1) \int_{0}^{\infty} d l & \left(\tilde{\mathcal{M}}_{\left(\frac{1}{4}, \frac{1}{4},-\frac{1}{2}\right)}^{(0)} \tilde{\mathcal{L}}[4,4]^{2} \tilde{\mathcal{L}}[8,2]-4 \tilde{\mathcal{M}}_{\left(\frac{1}{4}, \frac{1}{4},-\frac{1}{2}\right)}^{(3)}\right. \\
& \left.-4 \tilde{\mathcal{M}}_{\left(\frac{1}{4},-\frac{1}{4}, 0\right)}^{(2)} \tilde{\mathcal{L}}[4,4]+4 \tilde{\mathcal{M}}_{\left(\frac{1}{4}, \frac{1}{4},-\frac{1}{2}\right)}^{(1)}\right) .
\end{aligned}
$$

There are no extra factors whatsoever in the Möbius strip amplitude when switching to the ABB lattice. Summarizing, we get the following untwisted and 2-twisted tadpole cancellation conditions for the ABA model

$$
\frac{1}{8}\left(N_{\mu}-16\right)^{2}=0, \quad \operatorname{tr}\left(\gamma_{2 \mu}\right)^{2}=0
$$

When treating the twisted tadpole contribution individually for each $\mathrm{D} 6_{\mu}$-brane one realizes that the D6 $6_{1,3^{-}}$and $\mathrm{D} 6_{2,4}$-branes are charged under different twisted RR 5-forms. More precisely, the twisted sector tadpole condition receives contributions from the sixteen individual $\mathbb{Z}_{2}$ fixed points. Analyzing the intersections of the D6 ${ }_{\mu}$-branes with the 16 fixed points allows us to write each twisted sector tadpole condition as a sum of perfect squares. In order to satisfy all the conditions one really has to choose $\gamma_{2 \mu}$ traceless for all $\mu$.

For the ABB configuration the rank of the dual $B$-field is increased by 2 and the untwisted tadpole condition becomes

$$
\frac{1}{4}\left(N_{\mu}-8\right)^{2}=0
$$

Thus, the $\mathbb{Z}_{4}$ orientifold gives rise to two different models, one with gauge group of rank 16 and the other one with gauge group of rank 8 .

The massless closed string spectrum of the ABA model receives six chiral multiplets from the untwisted sector besides the graviton and dilaton multiplets. The 16 fixed points of $\Theta^{1,3}$ are all invariant under $\mathcal{R}$ and give rise to 16 chiral multiplets. Finally, the 16 fixed points of $\Theta^{2}$ need to be distinguished with respect to their mapping under $\Theta, \mathcal{R}$ and the combined action $\Theta \mathcal{R}$ providing another 15 chiral and 1 vectormultiplet.

Open strings in the $(\mu, \mu)$ sector carry eight massless states, which provide a vector multiplet in the gauge group $U(8) \times U(8)$, a chiral multiplet in the adjoint representation $((\mathbf{6 4}, \mathbf{1}) \oplus(\mathbf{1}, \mathbf{6 4}))$ and two chiral multiplets in the $((\mathbf{2 8}, \mathbf{1}) \oplus(\overline{\mathbf{2 8}}, \mathbf{1}) \oplus(\mathbf{1}, \mathbf{2 8}) \oplus(\mathbf{1}, \overline{\mathbf{2 8}}))$ representation. In the $(\mu, \mu+2)$ sector one has to take into account the extra minus signs in the loop channel Möbius strip amplitude, implying that compared to the $(\mu, \mu)$ sector both the $\Omega$ and the $\hat{\Theta}^{2}$ projection change sign. Considering also the multiplicity due to the twofold degeneracy of the ground state in this sector and an extra factor of two from the intersections between D $6_{\mu^{-}}$and $\mathrm{D} 6_{\mu+2}$-branes one gets two chiral multiplets in the $((\mathbf{6 4}, \mathbf{1}) \oplus(\mathbf{1}, \mathbf{6 4}))$ representation. Finally the $(\mu, \mu+1)$ strings only carry a single massless state to be counted with two orientations giving rise to one chiral multiplet in the $((\mathbf{8}, \overline{\mathbf{8}}) \oplus(\overline{\mathbf{8}}, \mathbf{8}))$ representation. Inspecting the massless open string spectrum reveals 
that it is not only nonchiral but surprisingly fits into $\mathcal{N}=2$ multiplets. However, the D-branes in the $(\mu, \mu+1)$ sector only preserve $\mathcal{N}=1$ supersymmetry, so that the appearance of $\mathcal{N}=2$ multiplets is purely accidental. The complete $\mathcal{N}=1$ supersymmetric massless spectrum is shown in the tables 4.2 and 4.4, where we have also summarized the results for the ABB model.

\begin{tabular}{|c|c|c|c|}
\hline Model & $(\mu, \mu)$ & $\begin{array}{c}(\mu, \mu+1) \\
+(\mu, \mu+3)\end{array}$ & $(\mu, \mu+2)$ \\
\hline \hline $\mathbb{Z}_{4} \mathbf{A B A}$ & $(\mathbf{6 4}, \mathbf{1}) \oplus(\mathbf{1}, \mathbf{6 4})$ & $(\mathbf{8}, \overline{\mathbf{8}}) \oplus(\overline{\mathbf{8}}, \mathbf{8})$ & $2(\mathbf{6 4}, \mathbf{1}) \oplus 2(\mathbf{1}, \mathbf{6 4})$ \\
$U(8) \times U(8)$ & $\oplus 2(\mathbf{2 8}, \mathbf{1}) \oplus 2(\overline{\mathbf{2 8}}, \mathbf{1})$ & & \\
& $\oplus 2(\mathbf{1}, \mathbf{2 8}) \oplus 2(\mathbf{1}, \mathbf{2 8})$ & & \\
$\mathbb{Z}_{4} \mathbf{A B B}$ & $(\mathbf{1 6}, \mathbf{1}) \oplus(\mathbf{1}, \mathbf{1 6})$ & $2(\mathbf{4}, \overline{\mathbf{4}}) \oplus 2(\overline{\mathbf{4}}, \mathbf{4})$ & $2(\mathbf{1 6}, \mathbf{1}) \oplus 2(\mathbf{1}, \mathbf{1 6})$ \\
$U(4) \times U(4)$ & $\oplus 2(\mathbf{6}, \mathbf{1}) \oplus 2(\overline{\mathbf{6}}, \mathbf{1})$ & & \\
& $\oplus 2(\mathbf{1}, \mathbf{6}) \oplus 2(\mathbf{1}, \overline{\mathbf{6}})$ & & \\
& \multicolumn{3}{|c}{} \\
\hline
\end{tabular}

Table 4.4: Open string spectra for $\mathbb{T}^{6} / \mathbb{Z}_{4}$ in $d=4$

\subsubsection{Results for other examples}

In this section we only make some remarks on the specialties of the other models which have been explicitly computed. The technical details of the computations are collected in the appendix.

The fourdimensional $\mathbb{Z}_{3}$ orientifold is very similar to the sixdimensional $\mathbb{Z}_{3}$. One is free to choose any of the four possible lattices $\{\mathbf{A A A}, \mathbf{A A B}, \mathbf{A B B}, \mathbf{B B B}\}$. In all four cases the D6-branes are located in the same way on all three $\mathbb{T}^{2}$. The lattice partition function for $\mathbf{A}^{3-i} \mathbf{B}^{i}$ leads to an overall factor of $3^{i}$ and the intersection numbers are also multiplied by $3^{i}$. In all cases the tadpole cancellation condition reads

$$
\frac{1}{2}\left(N_{\mu}-4\right)^{2}=0
$$

giving rise to an $S O(4)$ gauge symmetry, in accord with $\operatorname{rk}(B)=6$. The $\mathcal{N}=1$ supersymmetric spectra of all the models are collected in the tables 4.2 and 4.5 .

For each of the two $\mathbb{Z}_{6}$ orbifold groups we obtain two inequivalent models on the massless level. The two lattices of $\mathbf{A}$ and $\mathbf{B}$ type can be taken of the same kind as those for the $\mathbb{Z}_{3}$ orbifold in figure 4.1. The location of the D6-branes is chosen according to the general rules. The untwisted tadpole cancellation condition for either the $\mathbb{Z}_{6}$ or the $\mathbb{Z}_{6}^{\prime}$ orbifold is

$$
\frac{1}{2}\left(N_{\mu}-4\right)^{2}=0
$$

The 2-twisted tadpole condition is solved by traceless $\gamma_{3 \mu}$ matrices, leading to $U(2) \times U(2)$ gauge groups. Taking all twisted sectors and intersection points into account we derive 
the $\mathcal{N}=1$ supersymmetric open string massless spectrum shown in table 4.5. To distinguish $S U(2)$ singlets which are charged or neutral under the $U(1)$ we use the notation $\mathbf{1}$ and $\mathbf{1}_{\mathbf{0}}$. The $\mathbf{2}$ and $\overline{\mathbf{2}}$ are similarly distinguished by their abelian $U(1)$ charges.

Special care needs to be taken in the $\Theta^{2}$-twisted sector of the $\mathbb{Z}_{6}^{\prime}$ model, as the partition function of the bosonic zero-modes as well as the number of fixed points and intersections does depend on the factor of the gauge group. As a consequence the two choices $\mathbf{A B}$ and $\mathbf{B A}$ in the directions of odd $N v_{i}$ lead to slightly different models, which, on the massless level, boils down to an exchange of the two $U(2)$ factors. Note that the $\mathbb{Z}_{6}^{\prime}$ model is the only example where the exchange of the two factors is not a symmetry of the spectrum.

\begin{tabular}{|c|c|c|c|c|}
\hline Model & $(\mu, \mu)$ & $\begin{array}{c}(\mu, \mu+1) \\
+(\mu, \mu+N-1)\end{array}$ & $\begin{array}{c}(\mu, \mu+2) \\
+(\mu, \mu+N-2)\end{array}$ & $(\mu, \mu+3)$ \\
\hline $\begin{array}{c}\mathbb{Z}_{3} \text { AAA } \\
S O(4)\end{array}$ & $3(6)$ & $(\mathbf{1 0})$ & absent & absent \\
\hline $\begin{array}{c}\mathbb{Z}_{3} \text { AAB } \\
S O(4)\end{array}$ & $3(6)$ & $3(10)$ & absent & absent \\
\hline $\begin{array}{c}\mathbb{Z}_{3} \mathbf{A B B} \\
S O(4)\end{array}$ & $3(6)$ & $9(\mathbf{1 0})$ & absent & absent \\
\hline $\begin{array}{c}\mathbb{Z}_{3} \text { BBB } \\
S O(4)\end{array}$ & $3(6)$ & $27(\mathbf{1 0})$ & absent & absent \\
\hline $\begin{array}{c}\mathbb{Z}_{6} \text { ABA } \\
U(2) \times U(2)\end{array}$ & $\begin{array}{c}\left(\mathbf{4}, \mathbf{1}_{\mathbf{0}}\right) \oplus\left(\mathbf{1}_{\mathbf{0}}, \mathbf{4}\right) \\
\oplus 2\left(\mathbf{1}, \mathbf{1}_{\mathbf{0}}\right) \oplus 2\left(\overline{\mathbf{1}}, \mathbf{1}_{\mathbf{0}}\right) \\
\oplus 2\left(\mathbf{1}_{\mathbf{0}}, \mathbf{1}\right) \oplus 2\left(\mathbf{1}_{\mathbf{0}}, \overline{\mathbf{1}}\right)\end{array}$ & $(\mathbf{2}, \overline{\mathbf{2}}) \oplus(\overline{\mathbf{2}}, \mathbf{2})$ & $\begin{array}{c}\left(\mathbf{3}, \mathbf{1}_{\mathbf{0}}\right) \oplus\left(\overline{\mathbf{3}}, \mathbf{1}_{\mathbf{0}}\right) \\
\oplus\left(\mathbf{1}_{\mathbf{0}}, \mathbf{3}\right) \oplus\left(\mathbf{1}_{\mathbf{0}}, \overline{\mathbf{3}}\right) \\
\oplus 2\left(\mathbf{4}, \mathbf{1}_{\mathbf{0}}\right) \oplus 2\left(\mathbf{1}_{\mathbf{0}}, \mathbf{4}\right)\end{array}$ & $4(\mathbf{2}, \overline{\mathbf{2}}) \oplus 4(\overline{\mathbf{2}}, \mathbf{2})$ \\
\hline $\begin{array}{c}\mathbb{Z}_{6} \mathbf{A B B} \\
U(2) \times U(2)\end{array}$ & $\begin{array}{c}\left(\mathbf{4}, \mathbf{1}_{\mathbf{0}}\right) \oplus\left(\mathbf{1}_{\mathbf{0}}, \mathbf{4}\right) \\
\oplus 2\left(\mathbf{1}, \mathbf{1}_{\mathbf{0}}\right) \oplus 2\left(\overline{\mathbf{1}}, \mathbf{1}_{\mathbf{0}}\right) \\
\oplus 2\left(\mathbf{1}_{\mathbf{0}}, \mathbf{1}\right) \oplus 2\left(\mathbf{1}_{\mathbf{0}}, \overline{\mathbf{1}}\right)\end{array}$ & $3(\mathbf{2}, \overline{\mathbf{2}}) \oplus 3(\overline{\mathbf{2}}, \mathbf{2})$ & $\begin{array}{c}3\left(\mathbf{3}, \mathbf{1}_{\mathbf{0}}\right) \oplus 3\left(\overline{\mathbf{3}}, \mathbf{1}_{\mathbf{0}}\right) \\
\oplus 3\left(\mathbf{1}_{\mathbf{0}}, \mathbf{3}\right) \oplus 3\left(\mathbf{1}_{\mathbf{0}}, \overline{\mathbf{3}}\right) \\
\oplus 6\left(\mathbf{4}, \mathbf{1}_{\mathbf{0}}\right) \oplus 6\left(\mathbf{1}_{\mathbf{0}}, \mathbf{4}\right)\end{array}$ & $4(\mathbf{2}, \overline{\mathbf{2}}) \oplus 4(\overline{\mathbf{2}}, \mathbf{2})$ \\
\hline $\begin{array}{c}\mathbb{Z}_{6}^{\prime} \mathbf{A} \mathbf{A B} \\
U(2) \times U(2)\end{array}$ & $\begin{array}{c}\left(\mathbf{4}, \mathbf{1}_{\mathbf{0}}\right) \oplus\left(\mathbf{1}_{\mathbf{0}}, \mathbf{4}\right) \\
2\left(\mathbf{1}, \mathbf{1}_{\mathbf{0}}\right) \oplus 2\left(\overline{\mathbf{1}}, \mathbf{1}_{\mathbf{0}}\right) \\
\oplus 2\left(\mathbf{1}_{\mathbf{0}}, \mathbf{1}\right) \oplus 2\left(\mathbf{1}_{\mathbf{0}}, \overline{\mathbf{1}}\right)\end{array}$ & $2(\mathbf{2}, \overline{\mathbf{2}}) \oplus 2(\overline{\mathbf{2}}, \mathbf{2})$ & $\begin{array}{c}\left(\mathbf{1}, \mathbf{1}_{\mathbf{0}}\right) \oplus\left(\overline{\mathbf{1}}, \mathbf{1}_{\mathbf{0}}\right) \\
\oplus 3\left(\mathbf{1}_{\mathbf{0}}, \mathbf{1}\right) \oplus 3\left(\mathbf{1}_{\mathbf{0}}, \overline{\mathbf{1}}\right) \\
\oplus\left(\mathbf{4}, \mathbf{1}_{\mathbf{0}}\right) \oplus 3\left(\mathbf{1}_{\mathbf{0}}, \mathbf{4}\right)\end{array}$ & $4(\mathbf{2}, \overline{\mathbf{2}}) \oplus 4(\overline{\mathbf{2}}, \mathbf{2})$ \\
\hline $\begin{array}{c}\mathbb{Z}_{6}^{\prime} \mathbf{A B B} \\
U(2) \times U(2)\end{array}$ & $\begin{array}{c}\left(\mathbf{4}, \mathbf{1}_{\mathbf{0}}\right) \oplus\left(\mathbf{1}_{\mathbf{0}}, \mathbf{4}\right) \\
\oplus 2\left(\mathbf{1}, \mathbf{1}_{\mathbf{0}}\right) \oplus 2\left(\overline{\mathbf{1}}, \mathbf{1}_{\mathbf{0}}\right) \\
\oplus 2\left(\mathbf{1}_{\mathbf{0}}, \mathbf{1}\right) \oplus 2\left(\mathbf{1}_{\mathbf{0}}, \overline{\mathbf{1}}\right)\end{array}$ & $6(\mathbf{2}, \overline{\mathbf{2}}) \oplus 6(\overline{\mathbf{2}}, \mathbf{2})$ & $\begin{array}{c}3\left(\mathbf{1}, \mathbf{1}_{\mathbf{0}}\right) \oplus 3\left(\overline{\mathbf{1}}, \mathbf{1}_{\mathbf{0}}\right) \\
\oplus 9\left(\mathbf{1}_{\mathbf{0}}, \mathbf{1}\right) \oplus 9\left(\mathbf{1}_{\mathbf{0}}, \overline{\mathbf{1}}\right) \\
\oplus 3\left(\mathbf{4}, \mathbf{1}_{\mathbf{0}}\right) \oplus 9\left(\mathbf{1}_{\mathbf{0}}, \mathbf{4}\right)\end{array}$ & $4(\mathbf{2}, \overline{\mathbf{2}}) \oplus 4(\overline{\mathbf{2}}, \mathbf{2})$ \\
\hline
\end{tabular}

Table 4.5: Open string spectra for $\mathbb{T}^{6} / \mathbb{Z}_{N}, N=3,6,6^{\prime}$ in $d=4$

This completes our discussion of asymmetric orientifolds with D9- and D5-branes carrying magnetic fields on their world volume. 


\section{Chapter 5}

\section{Summary}

In this thesis the propagation of open strings on spaces with nontrivial gauge field backgrounds has been studied. The main focus was put on the compactification of type I superstrings on tori and toroidal orbifolds with a nonvanishing constant vacuum expectation value for the gauge field strength. In addition, a particular class of asymmetric orientifolds has been discussed, where D-branes with different magnetic fluxes got identified under the action of the orbifold group. There are various conceptually as well as phenomenologically attractive issues to be mentioned.

Already from pure field theoretical arguments one can deduce that the presence of nontrivial internal magnetic background fields induces an effective holonomy for the fields which are charged under the gauge group. In particular, charged fermions couple to the background field in a way that depends on their chirality and thus the resulting lower dimensional effective theory can be chiral even with a trivial background geometry such as a torus. At the same time the supersymmetry on the world volume of the D-branes may be broken at least partially by the vacuum expectation value of the field strength. Furthermore, gauge symmetry is reduced to the subgroup that commutes with the flux. Together these three properties are promising ingredients to construct nonsupersymmetric effective field theories from a Kaluza-Klein reduction on internal tori with extra magnetic fields tuned in a convenient fashion.

The field theoretical approach can then be generalized to compactifications of open string theory by providing microscopic definitions of D-branes with additional magnetic flux on their world volume. This is implemented by adjusting the boundary states that define the according open string sectors. These support the degrees of freedom of the gauge theory sector. The knowledge of the boundary states, combined with orientifold planes for type I theory, allows to compute the relevant amplitudes with boundary insertions in the world sheet. In principle, this provides a complete control over the perturbative aspects of magnetic background fields in open string theory.

By investigating boundary conditions one can notice a threefold identification of magnetic background fluxes on D-branes, noncommutative deformations of their world volume geometry as well as left-right asymmetric rotations of closed string coordinates applied to boundary conditions defining the D-branes in the world sheet CFT. The first 
part is well known: Open strings which propagate through standard commutative spacetime but in the presence of a constant magnetic background field behave exactly in the same way as in a noncommutative space without any background flux. The second part is more novel and exotic: The same effect can be obtained by rotating the closed string coordinates in an asymmetric way. Open strings subject to asymmetrically rotated boundary conditions are identical to such in a noncommutative space or with a background flux. This identification was employed to calculate certain properties of such theories in an efficient way by applying the asymmetric rotations to commutative quantities in order to get their noncommutative analogues.

The desired construction of low energy models with chiral fermion spectra, supersymmmetry breaking, interesting gauge groups and a matter content from tendimensional type I string theory starts from the requirement that the divergent contributions of massless fields in vacuum diagrams, the tadpoles, cancel. In the absence of supersymmetry the tadpoles of NSNS and RR fields cannot be canceled simultaneously. One has to tolerate the presence a dilaton tadpole and open string tachyons whose exact treatment is so far unknown. Still one can explore the perspectives of such an approach, which shows that the attractive features of the field theoretical approximation carry over to the fully fledged string models. A special version of T-duality leads towards a very intuitive interpretation of background fields in terms of geometrical moduli, angles between Dbranes and the shape of the background torus. This produces a nice visualization of the mechanism which allows to engineer gauge groups, spectra and other phenomenologically relevant aspects of the massless sector.

Several examples illustrate the simplicity and power of the approach. By just tuning the gauge fields in the desired way one can generate field theoretical models with the gauge group of the Standard Model or some grand unified generalization thereof. The spectra of chiral fermions can be chosen to contain any number of generations of fermions with quantum numbers adapted to those of the Standard Model. The bosonic spectrum contains scalar fields with negative squared masses, tachyons, which are suited to serve as Higgs bosons, if one assumes a stabilization mechanism. Altogether, a set of tools for the construction of semi-realistic low energy models has been developed. The most sophisticated version of such models so far includes magnetic flux as well as NSNS $B$ fields in the background of an orbifold of K3. The combination of nontrivial background geometry and nontrivial gauge fields provides the largest scope of freedom in designing type I vacua on tori and orbifolds, supersymmetric ones as well as nonsupersymmetric ones. The complete understanding of these models has also lead to some new insight into the role of the discrete NSNS $B$-field modulus in type I string theory in general.

A phenomenologically less relevant but very exotic relative of the type I orbifold models with background fields is the class of asymmetric orientifolds whose orbifold group acts in the open string sector by identifying different values for the magnetic field on the D-branes. The required symmetry of the background geometry under such asymmetric operations demands the presence of a nonvanishing $B$-field. It reduces the gauge group to a rather small rank, which, together with a nonchiral spectrum of fermions in four dimensions, delivers a poor perspective for a realistic scenario. The conceptual properties of these models are still worthwhile to be appreciated. They do produce fully consistent 
supersymmetric orientifolds of type II string theory, anomaly-free low energy models in six and four dimensions with an internal geometry where the distinction of commutative and noncommutative spaces is lost.

The scope of open string compactification including nontrivial gauge backgrounds is surely not exhausted so far. One could look for prototypes of models which allow to combine all the mentioned advantages in a single stroke and explore their phenomenology in greater detail. The position of these models in the web of string dualities is mostly unknown. Are there dual F-theoretic or heterotic models? Can one possibly extract nonperturbative information about the type I side? Is it possible to extrapolate the exact knowledge gained from the world sheet CFT to more generic points in the moduli space of type I compactifications? Can one include nonconstant flux related to alternative realizations of noncommutativity? Is it possible to give a more concrete interpretation to the tachyon condensation mechanism in these scenarios or gain more insight into the consequences of dilaton tadpoles, maybe even exact results? These questions do leave more than enough problems for future research. 


\section{Acknowledgements}

In the first place, I like to thank Dieter Lüst and Harald Dorn for supervising and supporting my work during the last nearly three years. I have also profited very much from the inspiring collaboration with various members of the string theory and quantum field theory group at the Humboldt Universität zu Berlin, Ralph Blumenhagen who taught me many things, Gottfried Curio, Lars Görlich, Albrecht Klemm and André Miemiec. Let me further only mention my PhD student colleagues Matthias Brändle, Volker Braun, Axel Krause, and Tassilo Ott among the many who were responsible for the excellent atmosphere and working conditions in the group. Finally, I am indebted to the Graduiertenkolleg "The Standard Model of Particle Physics - structure, precision tests and extensions" maintained by the DFG, the Studienstiftung des deutschen Volkes and the TMR and RTN networks "Quantum aspects of gauge theory, supersymmetry and unification", ERBFMRXCT96-0045, and "The quantum structure of spacetime and the geometric nature of fundamental interactions", HPRN-CT-2000-00131, for financial support. 
122 


\section{Appendix A}

\section{Definitions and notation}

In this appendix some basic definitions and notations are collected. They concern in particular the various expression relevant for the world sheet CFT of open and closed strings in flat background geometry and on orbifolds, the mode expansions of the coordinates and the Hamiltonian operators.

The bosonic closed string coordinates, solution to the twodimensional Laplace equation, are conveniently expanded in the form

$$
X^{\mu}(\tau, \sigma)=x^{\mu}+p^{\mu} \tau+w^{\mu} \sigma+\frac{i}{\sqrt{2}} \sum_{n \neq 0}\left(\frac{\alpha_{n}^{\mu}}{n} e^{-i n(\tau+\sigma)}+\frac{\tilde{\alpha}_{n}^{\mu}}{n} e^{-i n(\tau-\sigma)}\right)
$$

with a decomposition into left- and right-moving fields defined by

$$
\begin{aligned}
& X_{\mathrm{L}}^{\mu}(\tau+\sigma)=\frac{x_{\mathrm{L}}^{\mu}}{2}+\frac{p_{\mathrm{L}}^{\mu}}{2}(\tau+\sigma)+\frac{i}{\sqrt{2}} \sum_{n \neq 0} \frac{\alpha_{n}^{\mu}}{n} e^{i n(\tau+\sigma)}, \\
& X_{\mathrm{R}}^{\mu}(\tau-\sigma)=\frac{x_{\mathrm{R}}^{\mu}}{2}+\frac{p_{\mathrm{R}}^{\mu}}{2}(\tau-\sigma)+\frac{i}{\sqrt{2}} \sum_{n \neq 0} \frac{\tilde{\alpha}_{n}^{\mu}}{n} e^{i n(\tau-\sigma)} .
\end{aligned}
$$

Similarly, the fermionic coordinates and their decompositions are given by

$$
\begin{aligned}
\Psi^{\mu}(\tau, \sigma) & =\sum_{r}\left(\psi_{r}^{\mu} e^{-i r(\tau+\sigma)}+\tilde{\psi}_{r}^{\mu} e^{-i r(\tau-\sigma)}\right), \\
\Psi_{\mathrm{L}}^{\mu}(\tau+\sigma) & =\sum_{r} \psi_{r}^{\mu} e^{i r(\tau+\sigma)}, \\
\Psi_{\mathrm{R}}^{\mu}(\tau-\sigma) & =\sum_{r} \tilde{\psi}_{r}^{\mu} e^{i r(\tau-\sigma)},
\end{aligned}
$$

where the index $r$ runs over half-integers in the NS sector and over integers in the R sector. The commutator relations for the raising and lowering operators are

$$
\begin{aligned}
{\left[\alpha_{n}^{\mu}, \alpha_{m}^{\nu}\right] } & =\left[\tilde{\alpha}_{n}^{\mu}, \tilde{\alpha}_{m}^{\nu}\right]=n \delta_{n+m, 0} \delta^{\mu \nu} \\
\left\{\psi_{r}^{\mu}, \psi_{s}^{\nu}\right\} & =\left\{\tilde{\psi}_{r}^{\mu}, \tilde{\psi}_{s}^{\nu}\right\}=\delta_{r+s, 0} \delta^{\mu \nu}
\end{aligned}
$$

Each $\mathrm{R}$ groundstate then carries a representation of the Clifford algebra due to the fermionic zero-modes $\psi_{0}^{\mu}$ and $\tilde{\psi}_{0}^{\mu}$. For many purposes it is convenient to complexify the 
coordinates according to (2.18). The expansion of the complex fields reads

$$
\begin{aligned}
Z^{i}(\tau, \sigma)=z^{i}+\left(p_{\mathrm{L}}^{i}+p_{\mathrm{R}}^{i}\right) \tau+\left(p_{\mathrm{L}}^{i}-p_{\mathrm{R}}^{i}\right) \sigma & \\
& +\frac{i}{\sqrt{2}} \sum_{n \neq 0}\left(\frac{\alpha_{n}^{i}}{n} e^{-i n(\tau+\sigma)}+\frac{\tilde{\alpha}_{n}^{i}}{n} e^{-i n(\tau-\sigma)}\right), \\
\bar{Z}^{i}(\tau, \sigma)=\bar{z}^{i}+\left(\bar{p}_{\mathrm{L}}^{i}+\bar{p}_{\mathrm{R}}^{i}\right) \tau+\left(\bar{p}_{\mathrm{L}}^{i}-\bar{p}_{\mathrm{R}}^{i}\right) \sigma & \\
& -\frac{i}{\sqrt{2}} \sum_{n \neq 0}\left(\frac{\bar{\alpha}_{n}^{i}}{n} e^{-i n(\tau+\sigma)}+\frac{\tilde{\bar{\alpha}}_{n}^{i}}{n} e^{-i n(\tau-\sigma)}\right) .
\end{aligned}
$$

Expressions for the decomposition into left- and right-moving fields as well as the same set of formulas for the fermionic coordinates and finally the commutators are obtained completely analogously. The closed string Hamiltonian operator expressed in terms of the cartesian coordinates is

$$
\begin{aligned}
\mathcal{H}_{\mathrm{cl}}=\pi p^{2}+2 \pi \sum_{\mu}\left(\sum_{n=1}^{\infty}\left(\alpha_{-n}^{\mu} \alpha_{n}^{\mu}+\tilde{\alpha}_{-n}^{\mu} \tilde{\alpha}_{n}^{\mu}\right)\right. & \\
& \left.+\sum_{r>0}\left(r \psi_{-r}^{\mu} \psi_{r}^{\mu}+r \tilde{\psi}_{-r}^{\mu} \tilde{\psi}_{r}^{\mu}\right)\right)+2 \pi E_{0}
\end{aligned}
$$

which can easily be transformed into the complex basis. $E_{0}$ is the zero-point energy, obtained from (A.13) with $\delta=0$. The open string sectors are introduced by imposing boundary conditions, which imply relations for the creation and annihiliation operators, such as (2.4) and (2.3) for pure Dirichlet and Neumann boundary conditions. The resulting expansions for open string coordinates are very straightforward to be found. A slightly more complicated situation is a D-brane which carries constant magnetic flux $\mathcal{F}^{\mu \nu}$ on its world volume with boundary conditions given by (2.10). Imposing the same boundary conditions at both ends of the open string leads to an expression

$$
X^{\mu}(\tau, \sigma)=x^{\mu}+p^{\mu} \tau-\mathcal{F}^{\mu \nu} p_{\nu} \sigma+i \sum_{n \neq 0} \frac{1}{n}\left(\alpha_{n}^{\mu} \cos (n \sigma)+i \mathcal{F}_{\nu}^{\mu} \alpha_{n}^{\nu} \sin (n \sigma)\right) e^{-i n \tau} .
$$

The Fourier modings of the coordinates are still quantized in terms of integers and the flux only affects the mass spectrum of the bosonic zero-modes, which follows from the open string Hamiltonian

$$
\mathcal{H}_{\mathrm{op}}=\pi p^{2}+\pi \sum_{\mu}\left(\sum_{n=1}^{\infty} \alpha_{-n}^{\mu} \alpha_{n}^{\mu}+\sum_{r>0} r \psi_{-r}^{\mu} \psi_{r}^{\mu}\right)+\pi E_{0}
$$

On the contrary, when we consider an open string stretching between two D-branes with different fluxes, say $\mathcal{F}_{1}^{(1)}$ and $\mathcal{F}_{2}^{(1)}$ also the spectrum of oscillator excitations changes,

$$
\begin{aligned}
X^{1}(\tau, \sigma)=x^{1}-\sum_{n \in \mathbb{Z}} \frac{\alpha_{n+\delta}}{n+\delta} e^{-i(n+\delta) \tau} \sin \left((n+\delta) \sigma+\varphi_{1}\right) & \\
& +\sum_{m \in \mathbb{Z}} \frac{\alpha_{m}-\delta}{m-\delta} e^{-i(m-\delta) \tau} \sin \left((m-\delta) \sigma-\varphi_{1}\right),
\end{aligned}
$$




$$
\begin{aligned}
& X^{2}(\tau, \sigma)=x^{2}+i \sum_{n \in \mathbb{Z}} \frac{\alpha_{n+\delta}}{n+\delta} e^{-i(n+\delta) \tau} \sin \left((n+\delta) \sigma+\varphi_{1}\right) \\
&-i \sum_{m \in \mathbb{Z}} \frac{\alpha_{m-\delta}}{m-\delta} e^{-i(m-\delta) \tau} \sin \left((m-\delta) \sigma-\varphi_{1}\right) .
\end{aligned}
$$

The Hamiltonian (A.8) needs to be modified by just changing the oscillator modings accordingly

$$
\begin{aligned}
\mathcal{H}_{\mathcal{F}}=\pi p^{2}+\pi \sum_{\mu}( & \left.\sum_{n=1}^{\infty} \alpha_{-n}^{\mu} \alpha_{n}^{\mu}+\sum_{r>0} r \psi_{-r}^{\mu} \psi_{r}^{\mu}\right) \\
& +\sum_{n=1}^{\infty}\left(\alpha_{-n+\delta} \alpha_{n-\delta}+\alpha_{-n-\delta} \alpha_{n+\delta}\right) \\
& +\sum_{r>0}\left((r+\delta) \psi_{-r-\delta} \psi_{r+\delta}+(r-\delta) \psi_{-r+\delta} \psi_{r-\delta}\right)+\pi E_{0}
\end{aligned}
$$

$\delta$ is defined via

$$
e^{2 \pi i \delta}=-\frac{1+i f}{1-i f} \quad \text { with } \quad f=\frac{\mathcal{F}_{2}-\mathcal{F}_{1}}{1+\mathcal{F}_{1} \mathcal{F}_{2}}
$$

which is equivalent to

$$
\delta=\frac{\varphi_{2}-\varphi_{1}}{\pi} \quad \text { with } \quad \varphi_{\mu}=\arctan \left(\mathcal{F}_{\mu}\right)
$$

Simultaneously, the zero-point energy is shifted. For a complex boson with Fourier modings in $\mathbb{Z}+\delta$ it is given by

$$
E_{0}=\frac{1}{24}-\frac{1}{8}(2 \delta-1)^{2}
$$

and for a world sheet fermion the sign has to be switched. For convenience, we also display the coordinates for open strings which stretch between two D-branes that are located at a relative angle $\pi \delta$ in the plane spanned by $x^{1}$ and $x^{2}$,

$$
\begin{gathered}
X^{1}(\tau, \sigma)=x^{1}+i \sum_{n \in \mathbb{Z}} \frac{\alpha_{n+\delta}}{n+\delta} e^{-i(n+\delta) \tau} \cos \left((n+\delta) \sigma+\varphi_{1}\right) \\
+i \sum_{m \in \mathbb{Z}} \frac{\alpha_{m-\delta}}{m-\delta} e^{-i(m-\delta) \tau} \cos \left((m-\delta) \sigma-\varphi_{1}\right), \\
X^{2}(\tau, \sigma)=x^{2}+i \sum_{n \in \mathbb{Z}} \frac{\alpha_{n+\delta}}{n+\delta} e^{-i(n+\delta) \tau} \sin \left((n+\delta) \sigma+\varphi_{1}\right) \\
-i \sum_{m \in \mathbb{Z}} \frac{\alpha_{m-\delta}}{m-\delta} e^{-i(m-\delta) \tau} \sin \left((m-\delta) \sigma-\varphi_{1}\right) .
\end{gathered}
$$

They refer to the T-dual version where a left-moving reflection along $X^{2}$ has been performed. Very similar expansions are found for the coordinate fields of closed strings with twisted periodicity conditions (2.118) on an orbifold space. If we just look at a single 
complex plane and let the twist be given by a rotation by an angle $m \varphi^{\prime}$, the complexified coordinates of this $m$-twisted sector look

$$
\begin{array}{r}
Z(\tau, \sigma)=z_{0}+\frac{i}{\sqrt{2}} \sum_{n \neq 0}\left(\frac{\alpha_{n+m \delta^{\prime}}}{n+m \delta^{\prime}} e^{-i\left(n+m \delta^{\prime}\right)(\tau-\sigma)}\right. \\
\left.+\frac{\tilde{\alpha}_{n-m \delta^{\prime}}}{n-m \delta^{\prime}} e^{-i\left(n-m \delta^{\prime}\right)(\tau+\sigma)}\right), \\
\bar{Z}(\tau, \sigma)=\bar{z}_{0}+\frac{i}{\sqrt{2}} \sum_{n \neq 0}\left(\frac{\bar{\alpha}_{n-m \delta^{\prime}}}{n-m \delta^{\prime}} e^{-i\left(n-m \delta^{\prime}\right)(\tau-\sigma)}\right. \\
\left.+\frac{\tilde{\bar{\alpha}}_{n+m \delta^{\prime}}}{n+m \delta^{\prime}} e^{-i\left(n+m \delta^{\prime}\right)(\tau+\sigma)}\right),
\end{array}
$$

now defining $\delta^{\prime}=\varphi^{\prime} /(2 \pi)$. Again, the world sheet fermions have a perfectly analoguous expansion and the modified Hamiltonian in the $m$-twisted sector is

$\mathcal{H}_{\mathrm{orb}}^{(m)}=\pi p^{2}+2 \pi \sum_{\mu}\left(\sum_{n=1}^{\infty}\left(\alpha_{-n}^{\mu} \alpha_{n}^{\mu}+\tilde{\alpha}_{-n}^{\mu} \tilde{\alpha}_{n}^{\mu}\right)+\sum_{r>0}\left(r \psi_{-r}^{\mu} \psi_{r}^{\mu}+r \tilde{\psi}_{-r}^{\mu} \tilde{\psi}_{r}^{\mu}\right)\right) \quad$ (A.16)
$+\sum_{i}\left(\sum_{n=1}^{\infty}\left(\alpha_{-n-m \delta^{\prime}}^{i} \alpha_{n+m \delta^{\prime}}^{i}+\tilde{\alpha}_{-n+m \delta^{\prime}}^{i} \tilde{\alpha}_{n-m \delta^{\prime}}^{i}+\left(\delta^{\prime} \longrightarrow-\delta^{\prime}\right)\right)\right.$
$\left.+\sum_{r>0}\left(\left(r+m \delta^{\prime}\right) \psi_{-r-m \delta^{\prime}}^{i} \psi_{r+m \delta^{\prime}}^{i}+\left(r-m \delta^{\prime}\right) \tilde{\psi}_{-r+m \delta^{\prime}}^{i} \tilde{\psi}_{r-m \delta^{\prime}}^{i}+\left(\delta^{\prime} \longrightarrow-\delta^{\prime}\right)\right)\right)+2 \pi E_{0}^{(m)}$.

The commutation rules (A.4) also change in an obvious way. A more complete list of explicit expressions can for instance be found in [52]. 


\section{Appendix B}

\section{Annulus diagrams and modular transformation}

Being equipped with a CFT description of D-branes in terms of boundary states we can compute open string annulus diagrams which measure the forces which are excerted by D-branes upon each other. These are the lowest diagrams in the perturbative expansion of the string amplitude that can have interference with any closed string loop diagram, as the torus and the nonorientable Klein bottle. In type I string theory quantum anomalies occur whose cancellation leads to the consistency requirement of the tadpole cancellation which also involves the annulus diagram. Concretely, we go through the computation of annulus diagrams of open strings stretching between various D-branes in noncompact tendimensional space-time and with different magnetic fluxes on their world volume. We discuss certain technical details as a preliminary excercise for the later application to type I string theory.

The tree channel interpretation of the annulus amplitude of strings which have both ends on the same brane with flux $\mathcal{F}_{\mu}$ can be written as a transition function

$$
\tilde{\mathcal{A}}_{\mu \mu}=\int_{0}^{\infty} d l\left\langle\mathrm{D} p_{\mu}\left|e^{-l \mathcal{H}_{\mathrm{cl}}}\right| \mathrm{D} p_{\mu}\right\rangle
$$

of the boundary state $\left|\mathrm{D} p_{\mu}\right\rangle$ for the particular $\mathrm{D} p_{\mu}$-brane. The closed string Hamiltonian is given in (A.6). The expression describes the scattering of a closed string emitted from one boundary and annihilated into the second boundary on the same brane, $l$ measuring the "proper time" of the propagation. The amplitude is completely independent of the flux on the particular brane, which is not surprising in the light of the fact that a T-dual description can be given in terms of a lowerdimensional brane at some angle $\arctan \left(\mathcal{F}_{\mu}\right)$ relative to the coordinate axes, which is easily removed by a rotation of coordinates. Thus, the presence of a magnetic field on an isolated D-brane in noncompact space-time is merely a matter of convention.

As one figures the D-branes as static classical objects in the background of some string theory one calls such diagrams vacuum diagrams, tadpoles. In the effective field theory of the massless string modes, the D-branes enter as sources for fields which couple to their world volume $\mathcal{M}_{p+1}$, the dilaton of the NSNS sector and the appropriate tensor 
fields of the RR sector. In order to compute the diagram explicitly one needs to use the expressions given in section 2.1.2 to obtain the oscillator contributions of the two different spin structures on an annulus,

$$
\begin{aligned}
\mathrm{NSNS}\left\langle \pm \mid e^{-l \mathcal{H}_{\mathrm{cl}} \mid}\right\rangle_{\mathrm{NSNS}} & =\frac{\vartheta\left[\begin{array}{l}
0 \\
0
\end{array}\right]^{4}}{\eta^{12}} l^{(p-9) / 2}, \\
\mathrm{NSNS}\left\langle \pm\left|e^{-l \mathcal{H}_{\mathrm{cl}}}\right| \mp\right\rangle_{\mathrm{NSNS}} & =\frac{\vartheta\left[\begin{array}{c}
0 \\
1 / 2
\end{array}\right]^{4}}{\eta^{12}} l^{(p-9) / 2}, \\
\mathrm{RR}\left\langle \pm\left|e^{-l \mathcal{H}_{\mathrm{cl}}}\right| \pm\right\rangle_{\mathrm{RR}} & =-\frac{\vartheta\left[\begin{array}{c}
1 / 2 \\
0
\end{array}\right]^{4}}{\eta^{12}} l^{(p-9) / 2}, \\
\mathrm{RR}\left\langle \pm\left|e^{-l \mathcal{H}_{\mathrm{cl}}}\right| \mp\right\rangle_{\mathrm{RR}} & =\frac{\vartheta\left[\begin{array}{c}
1 / 2 \\
1 / 2
\end{array}\right]^{4}}{\eta^{12}} l^{(p-9) / 2}=0
\end{aligned}
$$

with argument $\tilde{q}=\exp (-4 \pi l)$. The prefactors with powers of $l$ stem from the integration over continuous momenta transverse to the $\mathrm{D} p$-brane, while the $\vartheta$ - and $\eta$-functions summarize the contributions of the oscillators and the zero-point energy (3.83). The entire amplitude finally reads

$$
\begin{aligned}
\tilde{\mathcal{A}}_{\mu \mu} & =\mathcal{N}_{\mu}^{2} \int_{0}^{\infty} d l l^{(p-9) / 2} \sum_{\alpha, \beta \in\{0,1 / 2\}}(-1)^{2(\alpha+\beta)} \frac{\vartheta\left[\begin{array}{l}
\alpha \\
\beta
\end{array}\right]^{4}}{\eta^{12}} \\
& =\mathcal{N}_{\mu}^{2}(1-1) \int_{0}^{\infty} d l l^{(p-9) / 2} \frac{\vartheta\left[\begin{array}{c}
1 / 2 \\
0
\end{array}\right]^{4}}{\eta^{12}}
\end{aligned}
$$

The last equation results from the Jacobi identity (D.3) for $\vartheta$-functions and ensures the vanishing of the amplitude as required by supersymmetry. It refers to the BPS nature of a D-brane, from which a "no-force" law derives. The normalization of the boundary state, the fixing of $\mathcal{N}_{\mu}$, is performed by comparing this expression to the loop channel and require the correct multiplicity of massless open string states. The modular transformation which provides the translation is given by $l=1 /(2 t)$. Using the formulas given in appendix $\mathrm{D}$, we get

$$
\tilde{\mathcal{A}}_{\mu \mu}=2^{5} \mathcal{N}_{\mu}^{2} \int_{0}^{\infty} \frac{d t}{(2 t)^{(p+3) / 2}} \frac{\vartheta\left[\begin{array}{c}
0 \\
1 / 2
\end{array}\right]^{4}}{\eta^{12}}
$$

with argument $q=\exp (-2 \pi t)$, which now needs to be compared to the loop channel amplitude

$$
\mathcal{A}_{\mu \mu}=\int_{0}^{\infty} \frac{d t}{t} \operatorname{Tr}_{\mathrm{NS}-\mathrm{R}}^{(\mu \mu)}\left(\mathcal{P}_{\mathrm{GSO}} e^{-2 t \mathcal{H}_{o p}}\right)
$$

the open string Hamiltonian given by (A.8). The trace is to be performed over the entire open string Hilbert space of $(\mu, \mu)$ open strings, counting space-time fermions with an extra minus sign. In particular, at the massless level, there are eight massless physical states in each sector, the vectormultiplet in ten dimensions. One then has to require the 
identity (2.16) of the two amplitudes (B.3) and (B.5) to ensure the normalization of the closed string boundary state which is consistent with its open string interpretation. In order to evaluate the traces over the open string spectrum, one uses (A.7) and (A.8) to derive

$$
\begin{aligned}
\operatorname{Tr}_{\mathrm{NS}}^{(\mu \mu)}\left(e^{-2 t \mathcal{H}_{o p}}\right) & =c \frac{\vartheta\left[\begin{array}{l}
0 \\
0
\end{array}\right]^{4}}{\eta^{12}}(2 t)^{-(p+1) / 2}, \\
\operatorname{Tr}_{\mathrm{R}}^{(\mu \mu)}\left(e^{-2 t \mathcal{H}_{o p}}\right) & =c \frac{\vartheta\left[\begin{array}{c}
1 / 2 \\
0
\end{array}\right]^{4}}{\eta^{12}}(2 t)^{-(p+1) / 2}, \\
\operatorname{Tr}_{\mathrm{NS}}^{(\mu \mu)}\left((-1)^{F} e^{-2 t \mathcal{H}_{o p}}\right) & =c \frac{\vartheta\left[\begin{array}{c}
0 \\
1 / 2
\end{array}\right]^{4}}{\eta^{12}}(2 t)^{-(p+1) / 2}, \\
\operatorname{Tr}_{\mathrm{R}}^{(\mu \mu)}\left((-1)^{F} e^{-2 t \mathcal{H}_{o p}}\right) & =c \frac{\vartheta\left[\begin{array}{c}
1 / 2 \\
1 / 2
\end{array}\right]^{4}}{\eta^{12}}(2 t)^{-(p+1) / 2}=0
\end{aligned}
$$

where $c$ is defined by

$$
c=\frac{V_{p+1}}{(2 \pi)^{p+1}}
$$

setting $\alpha^{\prime}=1$. The modular transformation thus relates the odd spin structures with different value for $\eta= \pm 1$ in the in- and out-state to the open string $\mathrm{R}$ sector, and the even spin structures to the open string NS sector. Vice versa, the insertion of $(-1)^{F}$ into the open string trace maps to the closed string RR sector, the insertion of the 1 to the NSNS sector. Combining the results for the loop channel amplitude, one gets

$$
\mathcal{A}_{\mu \mu}=c \int_{0}^{\infty} \frac{d t}{(2 t)^{(p+3) / 2}} \sum_{\alpha, \beta \in\{0,1 / 2\}}(-1)^{2(\alpha+\beta)} \frac{\vartheta\left[\begin{array}{l}
\alpha \\
\beta
\end{array}\right]^{4}}{\eta^{12}} .
$$

One can now read off the normalization factor for the closed string boundary state,

$$
\mathcal{N}_{\mu}^{2}=2^{-5} c
$$

independent of $\mathcal{F}_{\mu}$. This completes the definition of the boundary state of a $\mathrm{D} p$-brane in flat space-time, which now enables us to make an unambiguous computation of the annulus amplitude of strings with ends on any two different branes, in particular with additional flux on these branes. These amplitudes will depend on the values $\mathcal{F}_{\mu}$ of the fluxes on the branes, in accord with the fact T-duality maps this configuration to a set of two D-branes at a relative angle which cannot be removed by changing coordinates. In a toroidal compactification this angle is quantized and refers to the first Chern class of the gauge bundle. In addition, one can also turn on Wilson lines along the two circles of any torus. The annulus diagram of two branes wrapping a torus will then depend on these three data and in this sense capture the geometrical characterization of the gauge bundle on space-time in the world sheet CFT. The explicit computations to demonstrate this are sketched in chapter 2.4.4. 
In order to simplify the computation we specialize to the case where there are magnetic fields on two D9-branes, directed along the plane spanned by $x^{1}$ and $x^{2}$. We can then use the complexification (2.18) of coordinates, the two branes carrying magnetic fields $\mathcal{F}_{1}$ and $\mathcal{F}_{2}$. The tree channel expression for the annulus amplitude of open strings stretching between the two looks

$$
\tilde{\mathcal{A}}_{12}=\int_{0}^{\infty} d l\left\langle\mathrm{D} p_{1}\left|e^{-l \mathcal{H}_{\mathrm{cl}}}\right| \mathrm{D} p_{2}\right\rangle .
$$

In noncompact space-time the flux enters the boundary states only by the phase factors which were introduced into the coherent state (2.20) and by the absence of fermionic zero-modes in the $\mathrm{R}$ ground states. These phase factors directly translate into shifts of the arguments of the $\vartheta$-functions which summarize the contributions of the oscillators by $\delta=\left(\varphi_{2}-\varphi_{1}\right) / \pi$, setting $\varphi_{\mu}=\arctan \left(\mathcal{F}_{\mu}\right)$ again. The final result is

$$
\tilde{\mathcal{A}}_{12}=-2^{-4} \sin (\pi \delta) c \int_{0}^{\infty} d l \sum_{\alpha, \beta \in\{0,1 / 2\}}(-1)^{2(\alpha+\beta)} \frac{\vartheta\left[\begin{array}{c}
\alpha \\
\beta-\delta
\end{array}\right]}{\vartheta\left[\begin{array}{c}
1 / 2 \\
1 / 2-\delta
\end{array}\right]} \frac{\vartheta\left[\begin{array}{l}
\alpha \\
\beta
\end{array}\right]^{3}}{\eta^{9}} .
$$

Whenever the configuration of D-branes is supersymmetric, which clearly requires more than one nonvanishing direction for the fluxes $\mathcal{F}_{\mu}^{(i)}$, one can employ a generalized Jacobi identity (D.4) to show that the amplitude is vanishing. This matches perfectly with the conditions (2.30) for preserving supersymmetry in the effective theory.

In order to compute the amplitude (B.11) directly in the loop channel as a trace over open string states one needs the solution to the equations of motion subject to the boundary conditions (2.10), which is given by (A.9). The Hamiltonian is (A.10), appropriately omitting the zero-modes. The resulting loop channel annulus amplitude reads

$$
\mathcal{A}_{12}=2 i \sin (\pi \delta) c \int_{0}^{\infty} \frac{d t}{(2 t)^{(p+1) / 2}} \sum_{\alpha, \beta \in\{0,1 / 2\}}(-1)^{2(\alpha+\beta)} e^{-2 \pi i \alpha \delta} \frac{\vartheta\left[\begin{array}{c}
\delta-\beta \\
\alpha
\end{array}\right]}{\vartheta\left[\begin{array}{c}
\delta-1 / 2 \\
1 / 2
\end{array}\right]} \frac{\vartheta\left[\begin{array}{c}
\alpha \\
\beta
\end{array}\right]^{3}}{\eta^{9}}
$$

which is related to (B.11) by just the standard modular transformation $t=1 /(2 l)$. In a noncompact space the naive matching may sometimes no longer work and extra multiplicities need to be added in the loop channel which are automatically captured by the boundary state techniques in the tree channel. Geometrically these are the intersection numbers of the two respective cycles wrapped by the branes, which produce an extra prefactor for the trace of the open string spectrum. This is elaborated more explicitly in section 2.4.4. 


\section{Appendix $\mathrm{C}$}

\section{One-loop partition function in asymmetric orientifolds}

Here we provide the results for the one-loop amplitudes of the asymmetric orientifolds of chapter 4 and explain the abbbreviations used there. We compute first the loop diagrams of the Klein bottle, the annulus and the Möbius strip and convert the results into the tree channel. In general we denote the unitary action on Chan-Paton labels by $\gamma_{k \mu}$ and $\gamma_{\Omega k \mu}$ for $\hat{\Theta}^{k}$ and $\Omega \hat{\Theta}^{k}$ acting on the $\mathrm{D} 9_{\mu}$-branes respectively.

\section{C.1 Klein bottle}

The Klein bottle amplitude in the loop channel has the general form

$$
\mathcal{K}=2^{5-D} c_{D} \int_{0}^{\infty} \frac{d t}{t^{(6-D)}}\left(\frac{1}{4 N} \sum_{n, k=0}^{N} \mathcal{K}^{(n, k)} \mathcal{L}_{\mathcal{K}}^{(n, k)}\right)
$$

with $\mathcal{K}^{(n, k)}$ denoting the trace over oscillators (osc) in the sector twisted by $\hat{\Theta}^{n}$ with the insertion of $\hat{\Theta}^{k}$ inside

$$
\mathcal{K}^{(n, k)}=\operatorname{Tr}_{\text {osc }}^{(n)}\left(\Omega \hat{\Theta}^{k} \mathcal{P}_{\mathrm{GSO}} e^{-2 \pi t\left(L_{0}+\bar{L}_{0}\right)}\right)
$$

and $\mathcal{L}_{\mathcal{K}}^{(n, k)}$ standing for the trace over bosonic zero modes,

$$
\mathcal{L}_{\mathcal{K}}^{(n, k)}=\chi_{\mathcal{K}}^{(n, k)} \operatorname{Tr}_{\mathrm{KK}+\mathrm{W}}^{(n)}\left(\Omega \hat{\Theta}^{k} e^{-2 \pi t\left(L_{0}+\bar{L}_{0}\right)}\right) .
$$

Here $\chi_{\mathcal{K}}^{(n, k)}$ is the number of fixed points of $\Theta^{n}$, which are invariant under the operator $\mathcal{R} \Theta^{k}$ in the trace. Except for the $\mathbb{Z}_{6}^{\prime}$ orbifold these numbers, as well as the entire traces are equal for all insertions of $\hat{\Theta}^{k}$. We then omit the superscript $k$, writing $\mathcal{L}_{\mathcal{K}}^{(n)}, \mathcal{K}^{(n)}$ and $\chi_{\mathcal{K}}^{(n)}$. As a shorthand for the contributions of the bosonic zero modes we use

$$
\mathcal{L}[\alpha, \beta]=\left(\sum_{m \in \mathbb{Z}} e^{-\alpha \pi t m^{2} / R^{2}}\right)\left(\sum_{n \in \mathbb{Z}} e^{-\beta \pi t n^{2} / R^{2}}\right)
$$


having rescaled all radii to $R=R_{1}^{(i)}=R_{2}^{(i)}$. We always use the convention

$$
\sum_{\left(n v_{i}, k v_{i}\right) \notin \mathbb{Z}^{2}} n v_{i}=0
$$

for the $v_{i}$. The oscillator sums lead to the generic expression

$$
\mathcal{K}_{\left(v_{i}\right)}^{(n)}=(1-1) \frac{\vartheta\left[\begin{array}{c}
0 \\
1 / 2
\end{array}\right]}{\eta^{3}} \prod_{n v_{i} \notin \mathbb{Z}}\left(\frac{\vartheta\left[\begin{array}{c}
n v_{i} \\
1 / 2
\end{array}\right]}{\vartheta\left[\begin{array}{c}
1 / 2+n v_{i} \\
1 / 2
\end{array}\right]} e^{\pi i\left\langle n v_{i}\right\rangle}\right) \prod_{n v_{i} \in \mathbb{Z}}\left(\frac{\vartheta\left[\begin{array}{c}
0 \\
1 / 2
\end{array}\right]}{\eta^{3}}\right)
$$

with

$$
\left\langle n v_{i}\right\rangle=n v_{i}-\left[n v_{i}\right]-\frac{1}{2}
$$

$\mathcal{L}_{\mathcal{K}}^{(n, k)}$ has contributions whenever $n v_{i} \in \mathbb{Z}$. In contrast to the oscillator part we do not give any generic formula for the lattice sums, since one has to take into account which momentum and winding states are invariant under the respective operator in the trace. This does not only depend on the orbifold group action, as given by the $v_{i}$, but also on the orientation of the lattice with respect to the reflection $\mathcal{R}$. For the tree channel amplitudes we also define appropriate abbreviations:

$$
\begin{aligned}
\tilde{\mathcal{K}}_{\left(v_{i}\right)}^{(n)} & =(1-1) \frac{\vartheta\left[\begin{array}{c}
1 / 2 \\
0
\end{array}\right]}{\eta^{3}} \prod_{n v_{i} \notin \mathbb{Z}}\left(\frac{\vartheta\left[\begin{array}{c}
1 / 2 \\
n v_{i}
\end{array}\right]}{\vartheta\left[\begin{array}{c}
1 / 2 \\
1 / 2+n v_{i}
\end{array}\right]}\right) \prod_{n v_{i} \in \mathbb{Z}}\left(\frac{\vartheta\left[\begin{array}{c}
1 / 2 \\
0
\end{array}\right]}{\eta^{3}}\right), \\
\tilde{\mathcal{L}}[\alpha, \beta] & =\left(\sum_{m \in \mathbb{Z}} e^{-\alpha \pi l m^{2} R^{2}}\right)\left(\sum_{n \in \mathbb{Z}} e^{-\beta \pi l n^{2} R^{2}}\right),
\end{aligned}
$$

The expected prefactor to yield the complete projector is therefore

$$
\prod_{n v_{i} \notin \mathbb{Z}}\left(-2 \sin \left(\pi n v_{i}\right)\right)
$$

which just cancels the phase factors in the denominator of $\tilde{\mathcal{K}}_{\left(v_{i}\right)}^{(n)}$, whereas those in the numerator are physical due to (2.23).

\section{C.2 Annulus}

The open string diagrams can also be factorized into oscillator and momentum and winding parts. For the annulus the loop channel reads

$$
\mathcal{A}=c_{D} \int_{0}^{\infty} \frac{d t}{t^{(6-D)}}\left(\frac{1}{4 N} \sum_{n, k, i=0}^{N} \operatorname{tr}\left(\gamma_{k \mu}\right) \operatorname{tr}\left(\gamma_{k \mu}^{-1}\right) \mathcal{A}^{(n, k)} \mathcal{L}_{\mathcal{A}}^{(n, k, i)}\right)
$$

and analogously to the above we define

$$
\mathcal{A}^{(n, k)}=\operatorname{Tr}_{\text {osc }}^{(i, i+n)}\left(\hat{\Theta}^{k} \mathcal{P}_{\mathrm{GSO}} e^{-2 \pi t L_{0}}\right),
$$


where the trace is to be performed over the oscillator excitations of the open strings stretching between the $i$ and $i+n$ branes, with only $k \in\{0, N / 2\}$ contributions nonvanishing. Concerning the Chan-Paton matrices, we choose $\operatorname{tr}\left(\gamma_{0 \mu}\right)^{2}=N_{\mu}^{2}$, leaving only $\operatorname{tr}\left(\gamma_{(N / 2) \mu}\right)$ and the number of branes, $N_{\mu}$, to be determined by the tadpole cancellation conditions. The bosonic zero modes contribute

$$
\mathcal{L}_{\mathcal{A}}^{(n, k, \pm)}=\chi_{\mathcal{A}}^{(n, k, \pm)} \operatorname{Tr}_{\mathrm{KK}+\mathrm{W}}^{(i, i+n)}\left(\hat{\Theta}^{k} e^{-2 \pi t L_{0}}\right)
$$

$\chi_{\mathcal{A}}^{(n, k, \pm)}$ is the number of intersections of the two types of branes on the torus, which are invariant under $\Theta^{k}$. The trace (C.12) is only different from 1 if the $i$ and $i+n$ branes coincide on at least one torus $\mathbb{T}^{2}$ and $\hat{\Theta}^{k}$ acts trivially there. In the $\mathbb{Z}_{6}^{\prime}$ orbifold there is another distinction between odd and even $i$, which we have reserved the extra superscript \pm for. For the oscillator part we get the generic formula

$$
\mathcal{A}_{\left(v_{i}\right)}^{(n, k)}=(1-1) \frac{\vartheta\left[\begin{array}{c}
0 \\
1 / 2
\end{array}\right]}{\eta^{3}} \prod_{\left(n v_{i}, k v_{i}\right) \notin \mathbb{Z}^{2}}\left(\frac{2^{\delta} \vartheta\left[\begin{array}{c}
n v_{i} \\
1 / 2+k v_{i}
\end{array}\right]}{\vartheta\left[\begin{array}{l}
1 / 2+n v_{i} \\
1 / 2+k v_{i}
\end{array}\right]} e^{\pi i\left\langle n v_{i}\right\rangle}\right) \prod_{\left(n v_{i}, k v_{i}\right) \in \mathbb{Z}^{2}}\left(\frac{\vartheta\left[\begin{array}{c}
0 \\
1 / 2
\end{array}\right]}{\eta^{3}}\right) .
$$

The second product is empty, except if both $n v_{i}$ and $k v_{i}$ are integers, which is also the only case, in which there are contributions to $\mathcal{L}_{\mathcal{A}}^{(n, k, \pm)}$. We have introduced factors of $2^{\delta}$ for cancelling inappropriate factors occurring in the $\vartheta$-functions by defining $\delta=1$ if $n v_{i} \in \mathbb{Z}$ and $k v_{i} \in \mathbb{Z}+1 / 2$ and $\delta=0$ otherwise. One could have omitted these extra factors by using (3.63). Again there is no generic expression for the lattice sums and one needs to consider not only the type of brane as given by $i, n$ but also their orientation on the torus, in order to determine the normalization of momenta and winding states. $\mathcal{L}_{\mathcal{A}}^{(n, k, \pm)}$ can then be written in the form of (C.4), of course. For the tree channel oscillators we use

$$
\tilde{\mathcal{A}}_{\left(v_{i}\right)}^{(n, k)}=(1-1) \frac{\vartheta\left[\begin{array}{c}
1 / 2 \\
0
\end{array}\right]}{\eta^{3}} \prod_{\left(n v_{i}, k v_{i}\right) \notin \mathbb{Z}^{2}}\left(\frac{\vartheta\left[\begin{array}{c}
1 / 2+k v_{i} \\
n v_{i}
\end{array}\right]}{\vartheta\left[\begin{array}{c}
1 / 2+k v_{i} \\
1 / 2+n v_{i}
\end{array}\right]}\right) \prod_{\left(n v_{i}, k v_{i}\right) \in \mathbb{Z}^{2}}\left(\frac{\vartheta\left[\begin{array}{c}
1 / 2 \\
0
\end{array}\right]}{\eta^{3}}\right) .
$$

In the tree channel the annulus only contributes to the untwisted and $N / 2$-twisted sector, corresponding to the RR 10-form and a twisted RR 5-form tadpole.

\section{C.3 Möbius strip}

Finally we need to go through the Möbius strip amplitude. The loop channel expression is given by

$$
\mathcal{M}=-c_{D} \int_{0}^{\infty} \frac{d t}{t^{(6-D)}}\left(\frac{1}{4 N} \sum_{n, k, i=0}^{N} \operatorname{tr}\left(\gamma_{\Omega k \mu}^{-1} \gamma_{\Omega k \mu}^{\mathrm{T}}\right) \mathcal{M}^{(n, k)} \mathcal{L}_{\mathcal{M}}^{(n, k, i)}\right)
$$

with the oscillator

$$
\mathcal{M}^{(n, k)}=\operatorname{Tr}_{\text {osc }}^{(1,1+n)}\left(\Omega \hat{\Theta}^{k} \mathcal{P}_{\mathrm{GSO}} e^{-2 \pi t L_{0}}\right)
$$


and the zero mode trace

$$
\mathcal{L}_{\mathcal{M}}^{(n, k, \pm)}=\chi_{\mathcal{M}}^{(n, k, \pm)} \operatorname{Tr}_{\mathrm{KK}+\mathrm{W}}^{(i, i+n)}\left(\Omega \hat{\Theta}^{k} e^{-2 \pi t L_{0}}\right)
$$

Now $\chi_{\mathcal{M}}^{(n, k, \pm)}$ denotes the number of intersection points of the $i$ and $i+n$ branes, invariant under $\mathcal{R} \Theta^{k}$. By looking at the following chain of mappings of open strings

$$
\begin{aligned}
& (i, i+n) \stackrel{\Theta^{k}}{\longrightarrow}(i+2 k, i+n+2 k) \\
\stackrel{\mathcal{R}}{\longrightarrow} & (2-i-2 k, 2-i-n-2 k) \stackrel{\Omega}{\longrightarrow}(2-i-n-2 k, 2-i-2 k)
\end{aligned}
$$

one realizes that only strings that satisfy $2(k+i-1)+n=0 \bmod N$ can contribute in the Möbius strip. If $N$ is even, the relation has two solutions $k \equiv 1-i-n / 2,1-i-n / 2+N / 2$ for any combination of $i, n / 2 \in \mathbb{Z}$ and only one for any $i, n \in \mathbb{Z}$, if $N$ is odd. By regarding

$$
\Omega \hat{\Theta}^{1-i-n / 2}=\hat{\Theta}^{-(1-i) / 2}\left(\Omega \hat{\Theta}^{-n / 2}\right) \hat{\Theta}^{(1-i) / 2}
$$

one finds that $\Omega \hat{\Theta}^{1-i-n / 2}$ leaves the $(\mu, \mu+n)$ strings invariant just like $\Omega \hat{\Theta}^{-n / 2}$ the $(1,1+n)$ strings with ends on a D9-brane without any flux. The oscillator traces for all other values of $i$ are identical to the $i=1$ case, but the contributions arise from different $n, k$ combinations. We then get the formula

$$
\mathcal{M}_{\left(v_{i}\right)}^{(n, k)}=(1-1) \frac{\vartheta\left[\begin{array}{c}
1 / 2 \\
0
\end{array}\right]}{\eta^{3}} \prod_{\left(n v_{i}, k v_{i}\right) \notin \mathbb{Z}^{2}}\left(\frac{2^{\delta} \vartheta\left[\begin{array}{c}
1 / 2+n v_{i} \\
k v_{i}
\end{array}\right]}{\vartheta\left[\begin{array}{c}
1 / 2+n v_{i} \\
1 / 2+k v_{i}
\end{array}\right]} e^{\pi i\left\langle n v_{i}\right\rangle}\right) \prod_{\left(n v_{i}, k v_{i}\right) \in \mathbb{Z}^{2}}\left(\frac{\vartheta\left[\begin{array}{c}
1 / 2 \\
0
\end{array}\right]}{\eta^{3}}\right) .
$$

As for the annulus, there are lattice contributions if $n v_{i}$ and $k v_{i}$ are both integers. They differ from those of the annulus, as one needs to sum over states invariant under $\Omega$, which boils down to doubling the winding quantum numbers in the presence of nonvanishing NSNS $B$-field. For the modular transformed Möbius strip we use

$$
\tilde{\mathcal{M}}_{\left(v_{i}\right)}^{(m)}=(1-1) \frac{\vartheta\left[\begin{array}{c}
1 / 2 \\
0
\end{array}\right]}{\eta^{3}} \prod_{m v_{i} \notin \mathbb{Z}}\left(\frac{\vartheta\left[\begin{array}{c}
1 / 2 \\
m v_{i}
\end{array}\right]}{\vartheta\left[\begin{array}{c}
1 / 2 \\
1 / 2+m v_{i}
\end{array}\right]}\right) \prod_{m v_{i} \in \mathbb{Z}}\left(\frac{\vartheta\left[\begin{array}{c}
1 / 2 \\
0
\end{array}\right]}{\eta^{3}}\right) .
$$




\section{Appendix D}

\section{Useful formulas}

In this appendix we give basic definitions and fix some notation. We frequently employ the Jacobi $\vartheta$-function and Dedekind $\eta$-function

$$
\begin{aligned}
\vartheta\left[\begin{array}{l}
\alpha \\
\beta
\end{array}\right](q) & =\sum_{n \in \mathbb{Z}} q^{(n+\alpha)^{2} / 2} e^{2 \pi i(n+\alpha) \beta}, \\
\eta(q) & =q^{1 / 24} \prod_{n=1}^{\infty}\left(1-q^{n}\right)
\end{aligned}
$$

The argument $\alpha$ is defined modulo $\mathbb{Z}$ and in order to directly use the product expansion

$$
\frac{\vartheta\left[\begin{array}{l}
\alpha \\
\beta
\end{array}\right]}{\eta}(q)=e^{2 \pi i \alpha \beta} q^{\alpha^{2} / 2-1 / 24} \prod_{n=1}^{\infty}\left(\left(1+q^{n-1 / 2+\alpha} e^{2 \pi i \beta}\right)\left(1+q^{n-1 / 2-\alpha} e^{-2 \pi i \beta}\right)\right)
$$

one needs to choose $\alpha \in(-1 / 2,1 / 2]$. The standard Jacobi identity is

$$
\sum_{\alpha, \beta \in\{0,1 / 2\}}(-1)^{2(\alpha+\beta)} \vartheta\left[\begin{array}{l}
\alpha \\
\beta
\end{array}\right]=0 .
$$

It has a generalization to [115]

$$
\sum_{\alpha, \beta \in\{0,1 / 2\}}(-1)^{2(\alpha+\beta)} \prod_{i} \vartheta\left[\begin{array}{c}
\alpha \\
\beta+\delta^{(i)}
\end{array}\right]=0
$$

as long as

$$
\sum_{i} \delta^{(i)}=0
$$

Setting $q=\exp (-2 \pi t)$, the modular transformation $t \mapsto 1 / t$ is performed via

$$
\begin{aligned}
\vartheta\left[\begin{array}{c}
\alpha \\
\beta
\end{array}\right]\left(t^{-1}\right) & =\sqrt{t} e^{2 \pi i \alpha \beta} \vartheta\left[\begin{array}{c}
-\beta \\
\alpha
\end{array}\right](t), \\
\eta\left(t^{-1}\right) & =\sqrt{t} \eta(t)
\end{aligned}
$$


and by the Poisson resummation formula

$$
\sum_{n \in \mathbb{Z}} e^{-\pi n^{2} / t}=\sqrt{t} \sum_{n \in \mathbb{Z}} e^{-\pi n^{2} t} .
$$

In order to be able to apply (D.6) to the Möbius strip amplitude one can use

$$
\frac{\vartheta\left[\begin{array}{c}
\alpha+1 / 2 \\
\beta
\end{array}\right]}{\vartheta\left[\begin{array}{c}
\alpha+1 / 2 \\
\beta+1 / 2
\end{array}\right]}(-q)=e^{-\pi i \alpha} \frac{\vartheta\left[\begin{array}{c}
(\alpha+1) / 2 \\
\alpha / 2+\beta
\end{array}\right] \vartheta\left[\begin{array}{c}
\alpha / 2 \\
(\alpha+1) / 2+\beta
\end{array}\right]}{\vartheta\left[\begin{array}{c}
(\alpha+1) / 2 \\
(\alpha+1) / 2+\beta
\end{array}\right] \vartheta\left[\begin{array}{c}
\alpha / 2 \\
\alpha / 2+\beta
\end{array}\right]}\left(q^{2}\right)
$$

after restricting to $-1<\alpha \leq 0$. 


\section{Bibliography}

[1] M. B. Green, J. H. Schwarz, and E. Witten. Superstring Theory, two volumes. Cambridge University Press, 1987.

[2] D. Lüst and S. Theisen. Lectures on String Theory. Springer, 1989.

[3] J. Polchinski. String Theory, two volumes. Cambridge University Press, 1998.

[4] E. Witten. String Theory Dynamics in Various Dimensions. Nucl. Phys. B 443 (1995) 85.

[5] J. Dai, R. G. Leigh, and J. Polchinski. New Connections between String Theories. Mod. Phys. Lett. A Vol. 4 (1989) 2073.

[6] J. Polchinski. Dirichlet Branes and Ramond-Ramond Charges. Phys. Rev. Lett. 75 (1995) 4724.

[7] E. S. Fradkin and A. A. Tseytlin. Non-linear Electrodynamics from Quantized Strings. Phys. Lett. 163 B (1985) 123.

[8] R. G. Leigh. Dirac-Born-Infeld Action from Dirichlet $\sigma$-Model. Mod. Phys. Lett. A Vol. 4 (1989) 2773.

[9] C. Bachas. A Way to Break Supersymmetry. (hep-th/9503030).

[10] R. Blumenhagen, L. Görlich, D. Lüst, and B. Körs. Noncommutative Compactifications of Type I Strings on Tori with Magnetic Background Flux. JHEP 0010 (2000) 006.

[11] C. Angelantonj, I. Antoniadis, E. Dudas, and A. Sagnotti. Type I Strings on Magnetized Orbifolds and Brane Transmutation. Phys. Lett. B 489 (2000) 223.

[12] R. Blumenhagen, L. Görlich, B. Körs, and D. Lüst. Magnetic Flux in Toroidal Type I Compactification. (hep-th/0010198).

[13] C. Angelantonj and A. Sagnotti. Type I Vacua and Brane Transmutation. (hepth/0010279).

[14] R. Blumenhagen, D. Lüst, and B. Körs. Type I Strings with F- and B-flux. JHEP 0102 (2001) 030.

[15] A. Connes, M. R. Douglas, and A. Schwarz. Noncommutative Geometry and Matrix Theory: Compactification on Tori. JHEP 9802 (1998) 003. 
[16] M. R. Douglas and C. Hull. D-branes and the Noncommutative Torus. JHEP 9802 (1998) 008.

[17] V. Schomerus. D-branes and Deformation Quantization. JHEP 9906 (1999) 030.

[18] N. Seiberg and E. Witten. String Theory and Noncommutative Geometry. JHEP 9909 (1999) 032.

[19] R. Blumenhagen, L. Görlich, D. Lüst, and B. Körs. Asymmetric Orbifolds, Noncommutative Geometry and Type I String Vacua. Nucl. Phys. B 582 (2000) 44.

[20] J. Polchinski, S. Chaudhuri, and C. V. Johnson. Notes on D-Branes. (hepth/9602052).

[21] J. Polchinski. TASI Lectures on D-Branes. (hep-th/9611050).

[22] C. G. Callan, C. Lovelace, C. R. Nappi, and S. A. Yost. Adding Holes and Crosscaps to the Superstring. Nucl. Phys. B 293 (1987) 83.

[23] J. Polchinski and Y. Cai. Consistency of Superstring Theories. Nucl. Phys. B 296 (1988) 91.

[24] C. G. Callan, C. Lovelace, C. R. Nappi, and S. A. Yost. Loop Corrections to Superstring Equations of Motion. Nucl. Phys. B 308 (1988) 221.

[25] J. L. Cardy. Boundary Conditions, Fusion Rules and the Verlinde Formula. Nucl. Phys. B 324 (1989) 581.

[26] T. Onogi and N. Ishibashi. Conformal Field Theory on Surfaces with Boundaries and Crosscaps. Mod. Phys. Lett. A4 (1989) 161.

[27] N. Ishibashi. The boundary and Crosscap States in Conformal Field Theory. Mod. Phys. Lett. A4 (1989) 251.

[28] N. Seiberg and E. Witten. Spin Structures in String Theory. Nucl. Phys. B 276 (1986) 272.

[29] A. Sen. Stable Non-BPS Bound States of BPS D-branes. JHEP 9808 (1998) 10.

[30] E. Witten. Search for a Realistic Kaluza-Klein Theory. Nucl. Phys. B 186 (1981) 412.

[31] I. Antoniadis, E. Dudas, and A. Sagnotti. Brane Supersymmetry Breaking. Phys. Lett. B 464 (1999) 38.

[32] C. Angelantonj, I. Antoniadis, G. D'Appollonio, E. Dudas, and A. Sagnotti. Type I Vacua with Brane Supersymmetry Breaking. Nucl. Phys. B572 (2000) 36.

[33] M. Berkooz, M. R. Douglas, and R. G. Leigh. Branes Intersecting at Angles. Nucl. Phys. B 480 (1996) 265.

[34] A. Abouelsaood, C. G. Callan, C. R. Nappi, and S. A. Yost. Open Strings in Background Gauge Fiels. Nucl. Phys. B 280 (1987) 599. 
[35] C.-S. Chu. Noncommutative Open String: Neutral and Charged. (hep-th/0001144).

[36] N. K. Nielsen and P. Olesen. An Unstable Yang-Mills Field Mode. Nucl. Phys. B 144 (1978) 376.

[37] J. G. Russo and A. A. Tseytlin. Heterotic Strings in Uniform Magnetic Field. Nucl. Phys. B 454 (1995) 164.

[38] T. Eguchi, P. B. Gilkey, and A. J. Hansdon. Gravitation, Gauge Theories and Differential Geometry. Phys. Rept. 66 (1980) 213.

[39] A. Sen. Tachyon Condensation on the Brane Anti-Brane System. JHEP 9808 (1998) 12.

[40] A. Sen. Universality of the Tachyon Potential. JHEP 9912 (1999) 027.

[41] A. Sen and B. Zwiebach. Tachyon Condensation in String Field Theory. JHEP 0003 (2000) 002.

[42] E. Witten. BPS Bound States Of D0-D6 And D0-D8 Systems In A B-Field. (hepth/0012054).

[43] R. Blumenhagen, V. Braun, and R. Helling. Bound States of D $(2 p)$-D0 Systems and Supersymmetric p-Cycles. (hep-th/0012157).

[44] M. Sato. BPS bound States of D6-barnes and lowerdimensional D-branes. (hepth/0101226).

[45] B. Körs, D. Lüst, and A. Miemiec. Noncommuttaive D-brane and M-brane Bound States. (hep-th/0103203).

[46] B. Chen, H. Itoyama, T. Matsuo, and K. Murakami. p-p' Systems with B-field, Branes at Angles and Noncommutative Geometry. Nucl. Phys. B 576 (2000) 177.

[47] K. S. Narain, M. H. Samardi, and C. Vafa. Asymmetric Orbifolds. Nucl. Phys. B 288 (1987) 551.

[48] M. R. Douglas and B. Fiol. D-branes and Discrete Torsion 2. (hep-th/9903031).

[49] L. Dixon, J. A. Harvey, C. Vafa, and E. Witten. Strings on Orbifolds. Nucl. Phys. B $261(1985) 678$.

[50] L. Dixon, J. A. Harvey, C. Vafa, and E. Witten. Strings on Orbifolds (II). Nucl. Phys. B 274 (1986) 285.

[51] D. G. Markushevich, M. A. Olshanetsky, and A. M. Perelomov. Description of a Class of Superstring Compactifications Related Semi-Simple Lie Algebras. Commun. Math. Phys. 111, 247 (1987) 274.

[52] D.-E. Diaconescu and J. Gomis. Fractional Branes and Boundary States in Orbifold Theories. JHEP 0010 (2000) 001.

[53] A. Sagnotti. Strings on Orbifolds, Cargese '87. Ed. G. Mack et al. Pergamon Press $1988,521$. 
[54] P. Horava. Strings on World Sheet Orbifolds. Nucl. Phys. B 327 (1989) 461.

[55] P. Horava. Background Duality of Open String Models. Phys. Lett. B 231 (1989) 251.

[56] W. Fischler and L. Susskind. Dilaton Tadpoles, String Condensates and Scale Invariance. Phys. Lett. B 171 (1986) 383.

[57] W. Fischler and L. Susskind. Dilaton Tadpoles, String Condensates and Scale Invariance II. Phys. Lett. B 173 (1986) 262.

[58] E. Dudas and J. Mourad. Brane Solutions in Strings with Broken Supersymmetry and Dilaton Tadpoles. Phys. Lett. B 486 (2000) 172.

[59] R. Blumenhagen and A. Font. Dilaton Tadpoles, Warped Geometries and Large Extra Dimensions for Non-Supersymmetric Strings. (hep-th/0011269).

[60] L. Alvarez-Gaume and E. Witten. Gravitational Anomalies. Nucl. Phys. B 234 (1983) 269.

[61] M. Green, J. A. Harvey, and G. Moore. I-Brane Inflow and Anomalous Couplings on D-branes. Class. Quant. Grav. 14 (1997) 47.

[62] K. Dasguptar, D. P. Jatkar, and S. Mukhi. Gravitational Couplings and $\mathbb{Z}_{2}$ Orbifolds. Nucl. Phys. B 523 (1998) 465.

[63] K. Dasguptar and S. Mukhi. Anomaly Inflow on Orientifold Planes. JHEP 9803 (1998) 004.

[64] M. B. Green and J. H. Schwarz. Anomaly Cancellation in Supersymmetric D $=10$ Gauge Theory and Superstring Theory. Phys. Lett. B 149 (1984) 117.

[65] A. Sagnotti. A Note on the Green-Schwarz Mechanism in Open String Theories. Phys. Lett. B 294 (1992) 196.

[66] M. Dine, N. Seiberg, and E. Witten. Fayet-Iliopoulos Terms in String Theory. Nucl. Phys. B 289 (1987) 589.

[67] J. Erler. Anomaly Cancellation in Six Dimensions. J. Math. Phys. 35 (1994) 1819.

[68] B. Craps and F. Roose. Anomalous D-brane and Orientfold Couplings from the Boundary State. Phys. Lett. B 445 (1998) 150.

[69] J. F. Morales and C. A. Scrucca. Anomalous Couplings for D-branes and O-planes. Nucl. Phys. B 552 (1999) 291.

[70] M. Bianchi and J. F. Morales. Anomalies \& Tadpoles. JHEP 0003 (2000) 030.

[71] J. Lykken. Weak Scale Superstrings. Phys. Rev. D 54 (1996) 3693.

[72] N. Arkani-Hamed, S. Dimopoulos, and G. Dvali. The Hierarchy Problem and New Dimensions at a Millimeter. Phys. Lett. B 429 (1998) 263. 
[73] I. Antoniadis, N. Arkani-Hamed, S. Dimopoulos, and G. Dvali. New Dimensions at a Millimeter to a Fermi and Superstrings at a TeV. Phys. Lett. B 436 (1998) 257.

[74] N. Arkani-Hamed, S. Dimopoulos, and G. Dvali. Phenomenology, Astrophysics and Cosmology of Theories with Sub-Millimeter Dimensions and TeV Scale Quantum Gravity. Phys. Rev. D 59 (1999) 086004.

[75] I. Antoniadis and C. Bachas. Branes and the Gauge Hierarchy. Phys. Lett. B 450 (1999) 83.

[76] I. Antoniadis. String and D-brane Physics at Low Energy. (hep-th/0102202).

[77] R. N. Mohapatra and J. C. Pati. Left-Right Gauge Symmetry and an "isoconjugate" Model of CP Violation. Phys. Rev. D 11 (1975) 566.

[78] G. Aldazabel, S. Franco, L. E. Ibanez, R. Rabadan, and A. M. Uranga. D $=4$ Chiral String Compactfications from Intersecting Branes. (hep-th/0011073).

[79] L. E. Ibanez, H.-P. Nilles, and F. Quevedo. Reducing the Rank of the Gauge Group in Orbifold Compactifications of the Heterotic String. Phys. Lett. B 192 (1987) 332.

[80] E. G. Gimon and J. Polchinski. Consistency Conditions for Orientifolds and DManifolds. Phys. Rev. D 54 (1996) 1667.

[81] G. Aldazabel, S. Franco, L. E. Ibanez, R. Rabadan, and A. M. Uranga. Intersecting Brane Worlds. JHEP 0102 (12001) 047.

[82] A. M. Uranga. D-brane Probes, RR Tadpole Cancellation and K-theory Charge. Nucl. Phys. B 598 (2001) 225.

[83] M. Bianchi, G. Pradisi, and A. Sagnotti. Toroidal Compactification and Symmetry Breaking in Open-String Theories. Nucl. Phys. B 376 (1992) 365.

[84] M. Bianchi. A Note on Toroidal Compactifications of the Type I Superstring and Other Superstring Vacuum Configurations with 16 Supercharges. Nucl. Phys. B 528 (1998) 73.

[85] E. Witten. Toroidal Compactification Without Vector Structure. JHEP 9802 (1998) 006.

[86] Z. Kakushadze, G. Shiu, and S.-H. H. Tye. Type IIB Orientifolds with NS-NS Antisymmetric Tensor Backgrounds. Phys. Rev. D58 (1998) 086001.

[87] Z. Kakushadze. On Four Dimensional N=1 Type I Compactifications. Nucl. Phys. B 535 (1998) 311.

[88] C. Angelantonj. Comments on Open-String Orbifolds with a Non-Vanishing Bab. Nucl. Phys. B566 (2000) 126.

[89] Z. Kakushadze. Geometry of Orientifolds with NS-NS B-flux. Int. J. Mod. Phys. A15 (2000) 3113. 
[90] A. Sen. The Mirror Transform of Type I Vacua in Six Dimensions. Nucl. Phys. B 499 (1997) 45.

[91] Z. Kakushadze. Aspects of N=1 Type I-Heterotic Duality in Four Dimensions. Nucl. Phys. B 512 (1998) 221.

[92] Z. Kakushadze, G. Shiu, and S.-H. H. Tye. Type IIB Orientifolds, F-theory, Type I Strings on Orbifolds and Type I - Heterotic Duality. Nucl. Phys. B 533 (1998) 25.

[93] G. Pradisi and A. Sagnotti. Open String Orbifolds. Phys. Lett. B 216 (1989) 59.

[94] M. Bianchi and A. Sagnotti. On the Systematics of Open String Theory. Phys. Lett. B 247 (1990) 517.

[95] A. Dabholkar and J. Park. An Orientifold of Type IIB theory on K3. Nucl. Phys. B 472 (1996) 207.

[96] E. G. Gimon and C. V. Johnson. K3 Orientifolds. Nucl. Phys. B 477 (1996) 715.

[97] A. Dabholkar and J. Park. Strings on Orientifolds. Nucl. Phys. B 477 (1996) 701.

[98] M. Berkooz and G. Leigh. A $D=4 N=1$ Orbifold of Type I Strings. Nucl. Phys. B 483 (1997) 187.

[99] C. Angelantonj, M. Bianchi, G. Pradisi, A. Sagnotti, and Y. S. Stanev. Chiral Asymmetry in Four-Dimensional Open-String Vacua. Phys. Lett. B 385 (1996) 96.

[100] Z. Kakushadze and G. Shiu. A Chiral N=1 Type I Vacuum in Four Dimensions and Its Heterotic Dual. Phys. Rev. D 56 (1997) 3686.

[101] Z. Kakushadze and G. Shiu. 4-D Chiral N=1 Type I Vacua With And Without D5-Branes. Nucl. Phys. B 520 (1998) 75.

[102] G. Zwart. Four-dimensional $N=1 Z_{N} \times Z_{M}$ Orientifolds. Nucl. Phys. B 526 (1998) 378.

[103] L. E. Ibanez. A Chiral D=4, N=1 String Vacuum with a Finite Low-Energy Effective Field Theory. JHEP 9807 (1998) 002.

[104] G. Aldazabal, A. Font, L. E. Ibanez, and G. Violero. D=4, N=1, Type IIB Orientifolds. Nucl. Phys. B 536 (1998) 29.

[105] J. Polchinski. Tensors from K3 Orientifolds. Phys. Rev. D 55 (1997) 6423.

[106] M. Berkooz, R. G. Leigh, J. Polchinski, J. H. Schwarz, N. Seiberg, and E. Witten. Anomalies, Dualities and Topology of $D=6 \mathrm{~N}=1$ Superstring Vacua. Nucl. Phys. B 475 (1996) 115.

[107] R. Blumenhagen, L. Görlich, and B. Körs. Supersymmetric Orientifolds in 6D with D-branes at Angles. Nucl. Phys. B 569 (2000) 209.

[108] R. Blumenhagen, L. Görlich, and B. Körs. Supersymmetric 4D Orientifolds of Type IIA with D6-branes at Angles. JHEP 0001 (2000) 040. 
[109] R. Blumenhagen, L. Görlich, and B. Körs. A New Class of Supersymmetric Orientifolds with D-branes at Angles. (hep-th/0002146).

[110] M. Dine and E. Silverstein. New M-theory Backgrounds with Frozen Moduli. (hepth/9712166).

[111] R. Blumenhagen and L. Görlich. Orientifolds of Non-supersymmetric, Asymmetric Orbifolds. Nucl. Phys. B 551 (1999) 601.

[112] S. Förste, G. Honecker, and R. Schreyer. Supersymmetric $\mathbb{Z}_{N} \times \mathbb{Z}_{M}$ Orientifolds in $4 D$ with D-branes at Angles. Nucl. Phys. B593 (2001) 127.

[113] C. Angelantonj and R. Blumenhagen. Discrete Deformations in Type I Vacua. Phys. Lett. B 473 (2000) 86.

[114] G. Pradisi. Type I Vacua from Diagonal Z 3 Orbifolds. Nucl. Phys. B 575 (2000) 134.

[115] D. Mumford. Tata Lectures on Theta, two volumes. Birkhäuser, 1982 and 1984. 


\section{Selbständigkeitserklärung}

Hiermit erkläre ich, die vorliegende Arbeit selbständig ohne fremde Hilfe verfaßt zu haben und nur die angegebene Literatur und Hilfsmittel verwendet zu haben.

Boris Körs

5. Mai 2001 